HELGA GEBAUER

\title{
REGIONALE UMWELTNUTZUNGEN IN DER ZEIT
}




\section{HELGA GEBAUER}

\section{REGIONALE UMWELTNUTZUNGEN IN DER ZEIT}

Für Ökonomen ist es unbestritten, daß die Umwelt als Rohstofflieferant, Schadstoffaufnahmemedium und Standortfaktor dienen soll, jedoch sollen diese Dienste der Umwelt nicht kostenlos zur Verfügung gestellt werden. Es stellt sich also die Frage, wieviel „Umwelt“ ge- bzw. verbraucht werden kann. Damit ist ein Allokationsproblem definiert. Schadstoffe, die an die Umwelt abgegeben werden, akkumulieren sich z.T. im Zeitablauf; damit ist ein intertemporales Problem gegeben. Andererseits werden Schadstoffe durch Diffusionsvorgänge über Länder- und Regionengrenzen hinweggetragen; damit ist ein interregionales Problem gegeben. In dieser Abhandlung werden diese Probleme anhand eines Zwei-Regionen-Modells untersucht. Dabei wird die „Unterlieger-Oberlieger“Problematik unter unterschiedlichen institutionellen Arrangements diskutiert.

Helga Gebauer wurde 1955 in Heilbronn geboren. Studium der Volkswirtschaftslehre von 1973 bis 1979 an der Universität Mannheim. Von 1979 bis 1985 wissenschaftliche Mitarbeiterin beim Sonderforschungsbereich 5 „Staatliche Allokationspolitik im marktwirtschaftlichen System". 
Regionale Umweltnutzungen in der Zeit

Helga Gebauer - 978-3-631-75599-0

Downloaded from PubFactory at 01/11/2019 03:14:13AM

via free access 


\section{STAATLICHE ALLOKATIONSPOLITIK IM MARKTWIRTSCHAFTLICHEN SYSTEM}

Herausgegeben von

Klaus Conrad, Heinz König, Hans-Heinrich Nachtkamp, Rudiger Pethig, Ulrich Schlieper, Horst Siebert, Eberhard Wille

\section{Band 16}

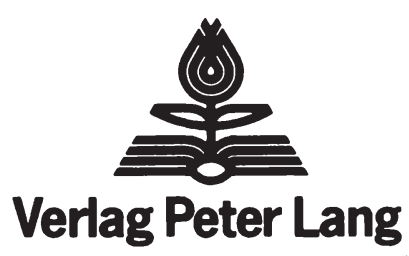

Frankfurt am Main · Bern · New York 


\section{HELGA GEBAUER}

\section{REGIONALE UMWELTNUTZUNGEN IN DER ZEIT}

Eine intertemporale Zwei-Regionen-Analyse

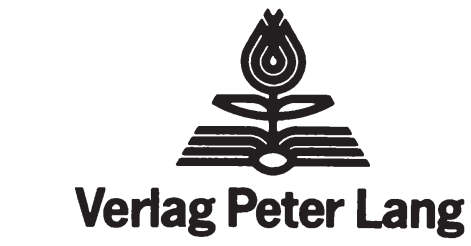

Frankfurt am Main · Bern · New York 
CIP-Kurztitelaufnahme der Deutschen Bibliothek

\section{Gebauer, Helga:}

Regionale Umweltnutzungen in der Zeit : e. intertemporale Zwei-Regionen-Analyse / Helga Gebauer. - Frankfurt am Main ; Bern ; New York : Lang, 1985. -

(Staatliche Allokationspolitik im marktwirtschaftlichen System ; Bd. 16)

ISBN 3-8204-8238-5

NE: GT

Open Access: The online version of this publication is published on www.peterlang.com and www.econstor.eu under the international Creative Commons License CC-BY 4.0. Learn more on how you can use and share this work: http://creativecommons. org/licenses/by/4.0.

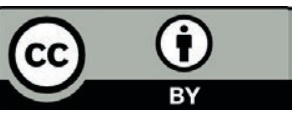

This book is available Open Access thanks to the kind support of ZBW - Leibniz-Informationszentrum Wirtschaft.

ISSN 0721-2860

ISBN 3-8204-8238-5

ISBN 978-3-631-75599-0 (eBook)

(C) Verlag Peter Lang GmbH, Frankfurt am Main 1985

Alle Rechte vorbehalten.

Nachdruck oder Vervielfältigung, auch auszugsweise, in allen Formen wie Mikrofilm, Xerographie, Mikrofiche, Mikrocard, Offset verboten.

Druck und Bindung: Weihert-Druck GmbH, Darmstadt 


\section{Meinen Eltern}


Helga Gebauer - 978-3-631-75599-0

Downloaded from PubFactory at 01/11/2019 03:14:13AM

via free access 


\section{Vorwort}

Für Ökonomen ist es unbestritten, daß die Umwelt als Rohstofflieferant, Schadstoffaufnahmemedium und Standortfaktor dienen soll, jedoch sollen diese Dienste der Umwelt nicht kostenlos zur Verfügung gestellt werden. Es stellt sich also die Frage, wieviel "Umwelt" ge- bzw. verbraucht werden kann. Damit ist ein Allokationsproblem definiert. Schadstoffe, die an die Umwelt abgegeben werden, haben z. T. die Eigenschaft, sich im Zeitablauf zu akkumulieren; damit ist ein intertemporales Problem gegeben. Andererseits werden Schadstoffe durch Diffusionsvorgänge über Länder- und Regionengrenzen hinweggetragen; damit ist ein interregionales Problem gegeben. In dieser Arbeit werden diese Probleme anhand eines Zwei-Regionen-Modells untersucht. Dabei wird die "Unterlieger-Oberlieger"-Problematik unter unterschiedlichen institutionellen Arrangements diskutiert. Die Arbeit wurde im November 1984 von der Fakultät für Volkswirtschaftslehre und Statistik der Universität Mannheim als Dissertation angenommen.

Mein Dank gilt vor allem meinem akademischen Lehrer Prof. Dr. Horst Siebert, der mir durch seine stete Diskussionsbereitschaft wertvolle Hinweise und Anregungen während der gesamten Entstehungszeit der Arbeit gegeben hat. Auch dem Korreferenten der Arbeit Prof. Dr. Klaus Conrad möchte ich sehr herzlich danken.

Die sehr stimulierende und international geprägte Atmosphäre des Mannheimer Sonderforschungsbereichs 5 "Staatliche Allokationspolitik im marktwirtschaftlichen System" trug wesentlich zum Gelingen der Dissertation bei. Teile der Arbeit wurden im SFB-Seminar vorgetragen; von den Teilnehmern habe ich Kritik und Anregungen erfahren, so daß ich diesen zu Dank verpflichtet bin. 
Von meinen Kollegen Dr. Ferdi Dudenhöffer, Sabine Toussaint, Dr. Wolfgang Vogt, Anke Meyer, Dr. Helmut Meder, Michael Rauscher, Andreas Seubert und Joachim Keck erhielt ich während der Entstehungszeit der Arbeit nicht nur fachliche Unterstützung, sondern auch das gute "Betriebsklima" steigerten meine Arbeitsmotivation.

Mannheim, im Dezember 1984

Helga Gebauer 


\section{Inhaltsverzeichnis}

Kapitel 1: Einführung

Kapitel 2: Grundlagen $\quad 5$

1. Problemstellung 5

2. Zur umweltökonomischen Einordnung der Arbeit 9

3. Ökologische Grundlagen 17

3.1 Das Ökosystem 17

3.2 Diffusion und Advektion 20

3.2.1 Grundzüge der Diffusionstheorie 20

3.2.2 Diffusions- bzw. Dispersionsmodelle 22

3.2.2.1 Der Energie- und Masseerhaltungsansatz 23

$\begin{array}{ll}\text { 3.2.2.2 Grundlegende Methoden } & 29\end{array}$

3.2.2.3 Die Lagrange-Formulierung $\quad 30$

3.2.2.4 Die Euler-Formulierung $\quad 37$

3.2.2.5 Abschließende Bemerkung $\quad 39$

Kapitel 3: Das Grundmodell $\quad 41$

1. Die Modellökonomie 41

1.1 Die Produktionstechnologie $\quad 42$

1.2 Das Ökosystem 44

1.3 Die Handelsbeziehungen $\quad 45$

1.4 Die Wohlfahrtsfunktionen 46

1.5 Die Optimierungsprobleme und die angewandten Methoden 50

1.5.1 Autonome Regionalbehörden $\quad 50$

1.5.2 Eine Zentralbehörde $\quad 52$

2. Grundzüge der intertemporalen Umweltallokation 54

2.1 Die Optimierungsprobleme und die Optimalbedingungen $\quad 54$

2.2 Die Abhängigkeit der Ökonomie von der Gegenwartsvorliebe $\quad 66$

2.3 Die Ausgestaltung der Emissionssteuern 66

3. Zusammenfassung 72

$\begin{array}{ll}\text { Anhang } 3 & 73\end{array}$ 
Kapitel 4: Interregionale Verflechtung durch Diffusion

1. Problemstellung

2. Autonome Regionalbehörden

2.1 Die Optimierungsprobleme und die Optimalbedingungen

2.2 Die zeitliche Entwicklung in Region 2

2.3 Die zeitliche Entwicklung in Region 1

2.4 Das Gesamt-Steady-State der Ökonomie

2.4.1 Die Ableitung des Gesamt-Steady-States

2.4.2 Auswirkungen von Parameteränderungen auf das

Steady-State

2.4.2.1 Die Auswirkungen der Höhe der sozialen

Zeitpräferenzrate

2.4.2.2 Die Auswirkungen der Höhe der

Assimilationskoeffizienten

2.4.2.3 Die Auswirkungen der Höhe der

Ressourcenausstattungen

2.4.2.4 Die Auswirkungen der Höhe des

Diffusionskoeffizienten

3. Zentralregierung

3.1 Das Optimierungsproblem und die Optimalbedingungen

3.2 Das Steady-State der Ökonomie

3.3 Die Auswirkungen von Parameteränderungen

3.3.1 Die Abhängigkeit von der sozialen Diskontrate

3.3.2 Die Abhängigkeit von den Assimilationskoeffizienten

3.3.3 Die Abhängigkeit von den Ressourcenausstattungen

3.3.4 Die Erhöhung des Diffusionskoeffizienten

4. Autonome Regionen mit zentraler Standardsetzung

4.1 Die Optimierungsprobleme

4.2 Die Auswirkungen von Parameteränderungen

4.2.1 Die Auswirkungen auf Region 2

4.2.2 Die Auswirkungen auf Region 1 
$\begin{array}{ll}\text { Anhang 4.II } & 136\end{array}$

$\begin{array}{ll}\text { Anhang 4.III } & 145\end{array}$

Kapitel 5: Interregionale Verflechtung durch Handel 151

1. Problemstellung 151

2. Die Optimierungsprobleme und die Optimalbedingungen 154

3. Die Ableitung der Optimalpfade und des Gleichgewichts 162

4. Die Auswirkungen von Parameteränderungen auf das Steady-State

4.1 Die Abhängigkeit des Steady-States von der sozialen Diskontrate und von den Assimilationskoeffizienten 165

4.2 Unterschiedliche Faktorausstattungen 166

4.3 Die Auswirkungen der Höhe des Relativpreises auf das $\begin{array}{lr}\text { Steady-State } & 169\end{array}$

5. Zusammenfassung 171

$\begin{array}{lr}\text { Anhang } 5 & 172\end{array}$

Kapitel 6: Interregionale Verflechtung durch Diffusion und Handel 177

$\begin{array}{ll}\text { 1. Problemstellung } & 177\end{array}$

2. Autonome Regionalbehörden 180

2.1 Die Optimierungsprobleme und die Optimalbedingungen $\quad 180$

2.2 Das Steady-State der Ökonomie 184

2.2.1 Die Ableitung für Region 2 184

2.2.2 Die Ableitung für Region $1 \quad 184$

2.2.3 Das Gesamt-Steady-State 186

2.3 Die Eigenschaften des Gleichgewichts $\quad 189$

2.3.1 Die Auswirkungen der Höhe der Diskontrate 189

2.3.2 Die Auswirkungen der Größe der Faktorausstattungen $\quad 190$

2.3.3 Die Auswirkungen der Höhe der Assimilationskoeffizienten 190

2.3.4 Die Auswirkungen der Höhe des Relativpreises 190

2.3.5 Die Auswirkungen der Höhe des Diffusionskoeffizienten 196 
3. Zentralbehörde 199

3.1 Das Optimierungsproblem und die Optimalbedingungen $\quad 199$

3.2 Die Auswirkungen der Größe der Parameter auf das

$\begin{array}{ll}\text { Steady-State } & 203\end{array}$

3.2.1 Die Auswirkungen von $\delta, \alpha_{i}$ und $\gamma_{2} \quad 203$

3.2.2 Die Auswirkungen der Höhe der Ressourcenausstattungen 204

3.2.4 Die Auswirkungen der Höhe des Relativpreises auf das Steady-State $\quad 205$

4. Autonome Regionalregierungen mit zentraler Standardsetzung 208

4.1 Das Optimierungsproblem für Region 2 und die

$\begin{array}{ll}\text { Optimalbedingungen } & 208\end{array}$

4.2 Die Auswirkungen der Größe der Parameter auf das Steady-State

$\begin{array}{lll}4.2 .1 & \text { Region } 2 & 215\end{array}$

$\begin{array}{lll}4.2 .2 \text { Region } 1 & 217\end{array}$

5. Zusammenfassung $\quad 218$

$\begin{array}{ll}\text { Anhang 6.I } & 221\end{array}$

$\begin{array}{ll}\text { Anhang 6.II } & 230\end{array}$

Anhang 6.II $\quad 241$

Kapitel 7: Schlußbetrachtung $\quad 249$

$\begin{array}{ll}\text { Literaturverzeichnis } & 253\end{array}$ 


\section{Verzeichnis zentraler Symbole}

\begin{tabular}{ll}
$a_{i}$ & Kuppelproduktionsfaktor der Region $i$ \\
$C_{i}$ & Konsum des Gutes $X_{i}$ \\
$E_{i}$ & Emissionen der Region $i$ \\
$\mathbf{E}_{i}$ & Emissionsstandard für Region $i$ \\
$f$ & Produktionsfunktion der Region $i$ \\
$H$ & Hamilton-Funktion \\
$i$ & Regionenindex \\
$L$ & Lagrange-Funktion \\
$M_{i}$ & Überschußnachfrage nach dem Faktor $R_{i}$ \\
$\mathbf{q}$ & konstanter Relativpreis \\
$R_{i}$ & Faktoreinsatz der Region $i$ \\
$\mathbf{R}_{i}$ & Faktorbestand der Region $i$ \\
$S_{i}$ & Immission der Region $i$ \\
$t$ & Zeit \\
$u^{i}$ & Nutzenfunktion der Region $i$ \\
$W_{i}$ & Wohlfahrtsfunktion der Region $i$ \\
$X_{i}$ & Produziertes Gut in Region $i$ \\
$a_{i}$ & Assimilationskoeffizient der Region $i$ \\
$\beta_{i}$ & Gewichtungsfaktor der Wohlfahrtsfunktion der Region $i$ \\
$Y_{i}$ & Diffusionskoeffizient der Region $i$ \\
$\delta$ & Soziale Zeitpräferenzrate \\
$\tau_{i}$ & Emissionssteuer in Region $i$ \\
\hline
\end{tabular}


Helga Gebauer - 978-3-631-75599-0

Downloaded from PubFactory at 01/11/2019 03:14:13AM

via free access 


\title{
Kapitel 1: Einführung
}

\begin{abstract}
"Allen verbalen Beteuerungen zum Trotz besitzt der Schutz der natürlichen Lebensgrundlagen in der praktischen Politik weder national noch international das gleiche Gewicht wie wirtschaftliche und soziale Belange. Wir haben den Schutz der menschlichen Gesundheit vor Umweltverschmutzungen, Lärm oder ionisierenden Strahlen durch vielfältige technische Maßnahmen verbessert. Von einer langfristigen Umweltvorsorge und einer haushälteri. schen Bewirtschaftung der knappen Umweltressourcen sind wir aber welt. weit soweit entfernt wie eh und je. Das Ende der Menschheit kommt vermutlich nicht mit einem militärischen Gewaltschlag. Es kommt unmerklich mit immer mehr Autos, mit der wachsenden Bequemlichkeit aus der Steckdose und mit der Überproduktion aus überzüchteter Monokultur. Die Natur ist ge duldig, Sie wird es noch für längere Zeit sein, wobei ihre Gefährdung für den, der sehen will, schon sichtbar ist. Ein Wald leistet lange Widerstand, bis er stirbt und sein Sterben offenkundig wird. Der Mensch kann unwahrschein. lich lange die Natur ausbeuten und gegen sie leben. Doch sagt uns eine jahr. tausendealte und immer gültige Erfahrung: Die Natur hat am Ende noch im. mergesiegt."
\end{abstract}

Hartkopf, Bohne (1983a, 3)

Der Konflikt zwischen dem materiellen Wohlstand der Menschen und den natürlichen Gegebenheiten - kurz Konflikt zwischen Ökonomie und Ökologie - verschärft sich in letzter Zeit zunehmend. Diesen Zwiespalt zwischen Ökonomie und der Lebensgrundlage der Menschen, Tiere und Pflanzen - der Natur - , gilt es zu verringern.

Für Ökonomen ist es unbestritten, daß die Umwelt als Produktionsfaktor, Schadstoffaufnahmemedium und Standortfaktor dienen soll, jedoch soll sie nicht kostenlos zur Verfügung gestellt werden. Es ist die optimale Menge an "Umwelt" zu finden, die ge- bzw. verbraucht werden kann. Ein Teilproblem in diesem Zusammenhang sind die Auswirkungen von Umweltverschmutzungen, die sich durch den Transport von Schadstoffen über weite Strecken hinweg ergeben. Dieser Sachverhalt ist in letzter Zeit unter den Schlagwörtern «Saurer Regen* und "Waldsterben* intensiv in öffentlichen Medien diskutiert worden und auch Gegenstand der politischen Diskussion in der Bundesrepublik Deutschland. In dieser Abhandlung werden die ökonomischen Auswirkungen des Schadstofftransports über Regionengrenzen hinweg in einem intertemporalen Kontext diskutiert. 
Anhand von zeitkontinuierlichen Optimierungsmodellen werden aus der Sicht vollständig informierter Planer mögliche Gleichgewichtszustände für einerseits autarke und andererseits für über Handelsbeziehungen verbundene Ökonomien analysiert, wobei die Natursysteme unterschiedliche Eigenschaften haben können. Insbesondere werden für eine Ökonomie, die aus zwei Regionen besteht, die Implikationen unterschiedlicher institutioneller Ausgestaltungen untersucht.

Im 2. Kapitel werden die Grundlagen der Arbeit dargestellt. Neben der Einordnung der Arbeit in die umweltökonomische Literatur werden die für diese Untersuchung relevanten ökologischen Zusammenhänge diskutiert, wobei insbesondere auf Diffusion und Advektion ${ }^{1)}$ von Schadstoffen abgestellt wird.

Im 3. Kapitel wird ein Grundmodell entwickelt, auf dem die weiteren Modelle aufbauen. Es werden die mathematischen und ökonomischen Implikationen dargestellt, die für die Diskussion der Modellvarianten der Kapitel 4,5 und 6 von Bedeutung sind.

Im 4. Kapitel wird die Diffusion von Schadstoffen von einer Region in die andere ("one way diffusion") ausführlich diskutiert. Dabei wird zum einen angenommen, daß die Regionen vollkommen eigenständig sind ["klassischer Föderalismus" (Frey (1977))]. Die zentralen Planer der beiden Regionen nehmen eine regionenegoistische Wohlfahrtsoptimierung vor. Diesem Modell wird das Modell einer Zentralbehörde gegenübergestellt, bei dem es einen zentralen Planer gibt, der die Gesamtwohlfahrt der Ökonomie unter Berücksichtigung der regionenspezifischen Eigenschaften maximiert. Schließlich wird untersucht, ob die Pareto-Eigenschaft des Zentralmodells auch für eigenständige Regionen erreicht werden kann, indem die Zentralregierung über die Setzung eines Emissionsstandards eingreift, während die zentralen Planer der Regionen weiterhin die Wohlfahrtsoptimierung vornehmen.

1) Unter Advektion versteht man den Prozeß, durch den Substanzen durch den Raum transportiert werden infolge der Bewegung der sie enthaltenden Medien. 
Im 5. Kapitel wird eine andere Art der Beziehung zwischen den Regionen in den Mittelpunkt der Betrachtung gestellt: Gegenüber dem Grundmodell des Kapitels 3 sind die Regionen nicht mehr autark, sondern über Handelsbeziehungen an den Weltmarkt angebunden. Dabei wird, um die Auswirkungen der Handelsbeziehungen in "reiner" Form analysieren zu können, von der Schadstoffdiffusion zwischen den Regionen abstrahiert.

Im 6. Kapitel schließlich werden die Modelle der Kapitel 4 und 5 integriert, um so die Beziehungen zwischen zwei Regionen unter Berücksichtigung der Umweltpolitik zu analysieren.

Im 7. Kapitel wird eine abschließende Bewertung der Ergebnisse gegeben. 
Helga Gebauer - 978-3-631-75599-0

Downloaded from PubFactory at 01/11/2019 03:14:13AM

via free access 


\section{Kapitel 2: Grundlagen}

\section{PROBLEMSTELLUNG}

Das Problem des «Sauren Regens» und des damit verbundenen «Waldsterbens" in Europa ist immer stärker in den Blickpunkt der öffentlichen Diskussion gekommen ${ }^{\prime \prime}$. Seit kurzem wird konstatiert, daß die Wälder im Schwarzwald und in Böhmen, im Harz und im Bayrischen Wald mit einer Gesch windigkeit sterben, die sich nicht allein mit Trockenheit oder Schädlingsbefall erklären läßt. Als Hauptursache dieses Waldsterbens wird der «Saure Regen * genannt ${ }^{2)}$. " Waldsterben * und «Saurer Regen * (sind) heute $z u$ dem größten Problem für die Umweltpolitik in der Bundesrepublik Deutschland geworden" [SRU(1983, 7)]. Dabei tritt ein Problem auf, das als neuartig bezeichnet werden kann: Nicht nur die in unmittelbarer Nähe von Industriezentren, Städten und großen Verkehrsanlagen (Autobahnen, Flugplätze) liegenden Wälder, sondern auch weitentfernte Erholungsgebiete werden geschädigt; "jetzt erscheint zumindest in Europa kein Gebiet mehr vor Schäden sicher" [SRU $(1983,7)]$.

Diese Entwicklung kommt für die Experten deshalb überraschend, da zwar schon in früheren Jahrhunderten «Rauchschäden ${ }^{3)}$ aufgetreten sind, man aber annahm, daß diese Schäden regional eng begrenzt sind und weiter entfernte Flächen ökologisch gesund bleiben. $\mathrm{Da} B$ dies nicht der Fall ist, darauf machten zuerst die skandinavischen Länder aufmerksam [vgl. z. B. Oden (1968)], wo die Seen in nichtindustrialisierten Zonen durch

1) Vgl. z. B. Der Spiegel Nr. 47/1981, Die ZEIT Nr. 34/1983.

2) «Saurer Regen* kann in diesem Fall als Synonym für Luftverschmutzung genommen werden, da nicht allein die Naßdeposition von Schwefel (Saurer Regen), sondern auch die Trockendeposition und vor allem auch andere Schadstoffe zu den Waldschäden führen [vgl. SRU (1983, 12-63)].

3) Schon von den "alten Römern" gibt es Quellen, die darauf schließen lassen, daß es Umweltprobleme gegeben hat [Siebert (1983a, 1) zitiert z. B. Seneca]. In London wurde Ende des 13. Jahrhunderts unter Edward I. eine "Royal Comission" für Luftverschmutzung gegründet [vgl. Osterkamp, Schneider (1982, 5)]. Ende des 19. Jahrhunderts traten in Deutschland vermehrt Umweltschäden durch die damals einsetzende Industrialisierung auf. Einen kurzen historischen Rūckblick über die Zusammenhänge von Luftverschmutzung und Waldsterben geben SRU (1983, 7-11), vgl. auch Siebert (1983a, 1-2). 
Säurebildung abstarben, deren Ursprung in Industriegebieten tausende Kilometer entfernt zu suchen war. Diese Entwicklung wurde von den anderen europäischen Ländern nicht allzu ernst genommen ${ }^{4}$. Erst seit Mitte der siebziger Jahre auch in diesen Ländern Schäden auftraten, ist die interregionale bzw. internationale Dimension des Umweltproblems Gegenstand der politischen Diskussion geworden.

"Die moderne Umweltdiskussion läßt die Erde (...) allen Menschen wohl zum ersten $\mathrm{Mal}$ in der modernen Geschichte als eng und begrenzt erscheinen" [Osterkamp, Schneider $(1982,6)]$.

Um einen quantitativen Eindruck der interregionalen Exporte von Schadstoffen zu vermitteln, wird im folgenden beispielhaft die Problematik der Schwefeldeposition über Ländergrenzen hinweg aufgezeigt.

In Tabelle 2.1 sind die Prozentanteile der täglichen Schwefelablagerungen nach Verursachern und Empfängern aufgelistet. Es fällt auf, daß nur zwei Länder über $50 \%$ der täglichen Schwefelkonzentration selbst verursachen. Die anderen sind alle mit unter $50 \%$ an ihrer eigenen Verschwefelung beteiligt. Im Extremfall Österreich sind zwei Staaten (Bundesrepublik Deutschland, CSSR) stärker an der täglichen Verschmutzung beteiligt als Österreich selbst.

Um zu sehen, mit welchen Schwefelmengen man es zu tun hat, sind in Tabelle 2.2 die tatsächlichen Depositionen nach Herkunfts- und Empfängerländern dargestellt.

Die letzte Zeile in Tabelle 2.2 gibt an, ob das jeweilige Land Netto"importeur" oder Netto"exporteur" an Schwefel ist. In der Bundesrepublik z. B. werden $138 \mathrm{kt}$ Schwefel mehr abgelagert pro Jahr als dort selbst produziert werden, während die DDR fast die Hälfte ihrer Gesamtproduktion an Schwefel an ihre Nachbarländer abgibt.

4) Vgl. Prittwitz (1983a und b). Prittwitz beleuchtet die Politik der Bundesrepublik Deutschland zum Problem der großrăumigen Luftverschmutzung unter dem Licht der aufgetretenen Schăden: "Erst als Anfang der achtziger Jahre großflăchige Waldschäden innerhalb der Bundesrepublik unübersehbar wurden und sich eine Art Volksbewegung gegen das Waldsterben entwickelte, änderte sich der Kurs der deutschen Behörden auf internationaler Ebene zu diesem Problem" [Prittwitz (1983b, 20)]. 


\begin{tabular}{|c|c|c|c|c|c|c|c|c|c|c|c|}
\hline $\begin{array}{l}\text { Empfänger } \\
\text { Verursacher }\end{array}$ & $D$ & DDR & $\mathbf{F}$ & $P L$ & CSSR & DK & NL & GB & $A$ & $s^{1)}$ & $\mathbf{N}^{1)}$ \\
\hline $\begin{array}{l}\text { Bundesrepublik } \\
\text { Deutschland }\end{array}$ & 44 & 19 & 10 & 6 & 7 & 15 & 22 & 3 & 15 & 9 & 11 \\
\hline DDR & 15 & 54 & 4 & 24 & 27 & 13 & 7 & 2 & 11 & 10 & 12 \\
\hline Frankreich & 11 & 3 & 48 & 2 & 2 & 5 & 10 & 3 & 4 & 3 & 5 \\
\hline Polen & 2 & 4 & 1 & 41 & 13 & 6 & 2 & 2 & 5 & 7 & 5 \\
\hline Tschechoslowakei & 8 & 10 & 2 & 14 & 38 & 4 & 3 & 1 & 21 & 4 & 5 \\
\hline Dalnemark & 1 & 1 & 0 & 1 & 0 & 29 & 1 & 1 & 0 & 13 & 7 \\
\hline Niederlande & 3 & 1 & 1 & 1 & 0 & 2 & 21 & 1 & 1 & 1 & 2 \\
\hline Großbritan Arland & 7 & 4 & 16 & 3 & 1 & 14 & 21 & 86 & 2 & 10 & 19 \\
\hline Osterreich & 1 & 0 & 0 & 1 & 2 & 0 & 0 & 0 & 14 & 0 & 0 \\
\hline Schweden ${ }^{1)}$ & 0 & 0 & 0 & 0 & 0 & 4 & 0 & 0 & 0 & 30 & 10 \\
\hline Norwegen ${ }^{11}$ & 0 & 0 & 0 & 0 & 0 & 1 & 0 & 0 & 0 & 5 & 16 \\
\hline $\begin{array}{l}\text { Totale Schwefelkon- } \\
\text { zentration } \mu S / m^{3} \text { (Ge- } \\
\text { samtmittel pro Land) }\end{array}$ & 13 & 16 & 6 & 11 & 20 & 4 & 11 & 5 & 9 & 2 & 1 \\
\hline
\end{tabular}

i) Süd-Schweden und Süd-Norwegen, Gebiete südlich $60^{\circ} \mathrm{n}$. Br. Quelle : BMI (1982 (90), 40)

Tabelle 2.1: Prozentanteil der Schwefelemissionen ausgewählter Verursacherstaaten an der mittleren täglichen Schwefelkonzentration in den Empfängerstaaten (Bezugsjahr 1978) 


\begin{tabular}{|c|c|c|c|c|c|c|c|c|c|c|c|c|c|c|c|c|c|c|c|c|c|}
\hline & $\begin{array}{l}\text { Empfänger } \\
\text { Verursacher }\end{array}$ & D & DDR & $\mathbf{F}$ & PL & CSSR & DK & NL & $B / L$ & GB & $s$ & $\mathrm{~N}$ & A & $\mathrm{CH}$ & HU & 1 & Ju & $\mathbf{E}$ & su & $\mathbf{R}$ & Summe \\
\hline & Bundesrepublik & 760 & 156 & 124 & 74 & 66 & 17 & 45 & 36 & 15 & 10 & 4 & 50 & 18 & 15 & 17 & 19 & 3 & 17 & 6 & 1392 \\
\hline & DDR & 196 & 480 & 39 & 360 & 323 & 12 & 9 & 7 & 7 & 10 & 4 & 30 & 4 & 27 & 11 & 28 & 1 & 42 & 11 & 1601 \\
\hline & frankreich & 167 & 18 & 827 & 16 & 14 & 4 & 19 & 57 & 22 & 3 & 2 & 11 & 29 & 4 & 28 & 6 & 39 & 5 & 2 & 1273 \\
\hline & Polen & 20 & 22 & 7 & 676 & 139 & 5 & 2 & 1 & 2 & 8 & 2 & 13 & 1 & 43 & 6 & 23 & 0 & 120 & 18 & 1108 \\
\hline & Tschechoslowakei & 90 & 71 & 20 & 196 & 459 & 4 & 3 & 2 & 3 & 4 & 1 & 67 & 4 & 132 & 14 & 64 & 1 & 46 & 37 & 1222 \\
\hline & Dänemark & 10 & 10 & 1 & 13 & 1 & 43 & 1 & 0 & 2 & 24 & 4 & 0 & 0 & 0 & 0 & 0 & 0 & 6 & 0 & 115 \\
\hline ס & Niederlande & 48 & 8 & 17 & 6 & 3 & 2 & 48 & 15 & 7 & 1 & 1 & 1 & 1 & 1 & 1 & 1 & 1 & 2 & 0 & 164 \\
\hline כ) & Belgien./Luxemburg & 95 & 12 & 76 & 8 & 6 & 3 & 24 & 53 & 7 & 2 & 1 & 3 & 2 & 1 & 2 & 2 & 1 & 2 & 1 & 301 \\
\hline$\frac{0}{1}$ & GroBbritan./Irland & 94 & 25 & 201 & 26 & 10 & 17 & 46 & 35 & 827 & 11 & 10 & 5 & 4 & 3 & 3 & 5 & 6 & 10 & 2 & 1340 \\
\hline $\overrightarrow{0}$ & S-Schweden & 1 & 1 & 0 & 5 & .0 & 4 & 0 & 0 & 1 & 55 & 5 & 0 & 0 & 0 & 0 & 0 & 0 & 7 & 0 & 79 \\
\hline & S-Norwegen & 0 & 0 & 0 & 1 & 0 & 1 & 0 & 3 & 0 & 8 & 10 & 0 & 0 & 0 & 0 & 0 & 0 & 1 & 0 & 21 \\
\hline$\frac{1}{\Phi}$ & Osterreich & 10 & 3 & 3 & 5 & 18 & 0 & 0 & 0 & 0 & 0 & 0 & 54 & 2 & 27 & 13 & 36 & 0 & 2 & 4 & 177 \\
\hline రิ & Schweiz & 8 & 0 & 7 & 1 & 1 & 0 & 0 & 0 & 0 & 0 & 0 & 3 & 17 & 0 & 9 & 1 & 0 & 0 & o & 47 \\
\hline D & Ungarn & 4 & 3 & 3 & 32 & 41 & 1 & 0 & 0 & 0 & 1 & 0 & 13 & 1 & 229 & 8 & 120 & 0 & 22 & 101 & 579 \\
\hline$\stackrel{\circ}{\rightleftharpoons} \stackrel{\mathbb{\Phi}}{=}$ & $\mathrm{N}$-Italien & 18 & 2 & 67 & 7 & 10 & 1 & 0 & 0 & 1 & 1 & 0 & 33 & 48 & 13 & 904 & 76 & 1 & 3 & 6 & 1191 \\
\hline$\vec{N} \varphi$ & Jugoslawien & 6 & 4 & 6 & 19 & 21 & 1 & 0 & 0 & 1 & 1 & 0 & 29 & 2 & 94 & 47 & 715 & 0 & 9 & 64 & 1018 \\
\hline$\leq \overrightarrow{0} \dot{\omega}$ & N-Spanien & 1 & 0 & 24 & 0 & 0 & 0 & 0 & 0 & 1 & 0 & 0 & 0 & 0 & 0 & 1 & 0 & 72 & 0 & 0 & 99 \\
\hline & W-RuBland & 0 & 0 & 0 & 11 & 2 & 0 & 0 & 0 & 0 & 2 & 0 & 0 & 0 & 2 & 0 & 1 & 0 & 76 & 8 & 102 \\
\hline 華 节 & Rumănien & 0 & 0 & 1 & 6 & 4 & 0 & 0 & 0 & 0 & 0 & 0 & 1 & 0 & 16 & 1 & 45 & 0 & 9 & 124 & 207 \\
\hline $\begin{array}{l}W_{0} \\
\text { 足 }\end{array}$ & Gesamtdeposition & 1530 & 817 & 1424 & 1462 & 1117 & 117 & 199 & 206 & 897 & 141 & 46 & 314 & 134 & 607 & 1065 & 1140 & 125 & 377 & 384 & \\
\hline & $\begin{array}{l}\text { Emissions-/ } \\
\text { Depositionsbilanz }\end{array}$ & -138 & +784 & -151 & .354 & +105 & -2 & -39 & +95 & +443 & .62 & .25 & -137 & -87 & .28 & +126 & -122 & .26 & .275 & |-177 & \\
\hline
\end{tabular}




\section{ZUR UMWELTÖKONOMISCHEN EINORDNUNG DER ARBEIT}

"Die Umwelt als Gesamtheit der den menschlichen Lebensraum definierenden Gegebenheiten wurde von Ökonomen lange Zeit ignoriert. Luft und Wasser waren die Prototypen freier Güter - ohne Preis, nicht knapp und damit nicht Gegenstand des Wirtschaftens" [Siebert (1979b,2)]. Erst als Mitte bis Ende der sechziger Jahre die Umweltschäden merklicher wurden, ist ein sprunghafter Anstieg der umweltökonomischen Literatur zu verzeichnen ${ }^{51}$.

Die theoretische Grundlage legte A. C. Pigou (1932) mit seiner auf Marshall (1898) zurückgehenden Theorie der (technologischen) externen Effekte, die Kneese $(1962,1964)$ zum ersten Mal auf umweltökonomische Tatbestände anwandte [vgl. Fisher, Peterson $(1976,12)]$. Für Markt-Ökonomien existiert bei Vorliegen von externen Effekten kein gesellschaftliches Wohlfahrtsoptimum, da die einzelwirtschaftlichen Kosten nicht mit den gesellschaftlichen Kosten übereinstimmen ${ }^{6}$, d. h. die ökonomische Theorie sagt uns, daß, wenn eine Aktivität eine negative Externalität (wie den sauren Regen) mit sich bringt, ein freier Markt zu viel dieser Aktivität erzeugt im Vergleich zum gesellschaftlichen Optimum [vgl. Forster $(1982,2)]$.

Die Umwelt hat für das ökonomische System die folgenden vier Funktionen: Öffentliches Konsumgut, Lieferant von Rohstoffen und Boden, Aufnahmemedium von Schadstoffen [Siebert $(1978,14)]^{7}$. Hier ist eine Naht-

5) Bis dahin war die Umwelt, besser die Natur, vor allem als Lieferant von Rohstoffen betrachtet worden. Malthus (1798) und Ricardo (1817) zeichneten ein beāngstigendes Scenario begrenzten Ackerlandes und einer wachsenden Bevölkerung. Jevons (1865) untersuchte die Endlichkeit der Kohlevorkommen in England und sagte ein Ende der industriellen Revolution voraus. Mill (1865) war der erste, der die Umwelt als Garant von Lebensqualität und als öffentliches Konsumgut betrachtete.

In neuerer Zeit zeichnete der Bericht des Club of Rome ein ähnlich beängstigendes Scenario für die Begrenztheit des "Raumschiffs" Erde, vgl. Meadows et al (1972).

Einen Überblick über die umweltökonomische Literatur bis 1975 geben Fisher, Peterson (1976).

6) Für eine Diskussion der Theorie der externen Effekte vgl. Mishan (1971); in umweltökonomischem Zusammenhang vgl. z. B. Bender (1976, 24-33), Siebert (1978, 30ff).

7) Vgl. hierzu auch Victor (1972, 37-47), sowie die ausführliche Beschreibung der Funktionen der Umwelt für das ökonomische System von Siebert (1978, 8-12). 
stelle zwischen Ökonomie und Naturwissenschaften gegeben, da physikalische, chemische, biologische, meteorologische u. a. Gegebenheiten als Restriktionen für ein ökonomisches System berücksichtigt werden, müssen. Diese Synthese von Ökonomie und Naturwissenschaften wurde, bis auf wenige Ausnahmen ${ }^{8}$, bis jetzt nur rudimentär betrieben. "In many respects economic theory has lost its biophysical foundation" [Faber (1984, 2)].

Die Umwelt als Lieferant von Rohstoffen ist ein eigenständiger Zweig der Ökonomie der Naturressourcennutzung, die vor allem durch die sogenannten Ölkrisen eine starke Beachtung gefunden hat. Einen Überblick über die ressourcenökonomische Literatur geben Peterson, Fisher (1977). Siebert (1983b) gibt einen Überblick über die neuere Literatur.

Die Umwelt als öffentliches Konsumgut ${ }^{9)}$ wird in den nachfolgenden Modellen dadurch berücksichtigt, daß der Immissionsbestand in die Wohlfahrtsfunktionen ${ }^{10)}$ mit eingeht: Je größer der Immissionsbestand ist, desto niedriger ist c. p. die Wohlfahrt in der betrachteten Ökonomie. Dieser Vorgehensweise wird von vielen Autoren gefolgt, vgl. z. B. für statische Modelle Siebert (1978 und 1981), Pethig (1979), Van Zele (1978) und für dynamische Modelle Keeler et al (1972), Plourde (1972), Smith (1972), d'Arge, Kogiku (1973), Forster (1973), Mäler (1974), Schulze (1974), Weinstein, Zeckhauser (1974), Vogt (1981).

In dem hier angesprochenen Fall sind die Auswirkungen auf die Umwelt-

8) Vgl. z. B. Kneese et al (1970), die das Masseerhaltungsgesetz in die Umweltökonomie einführten. Einen anderen Ansatz wählte Georgescu-Roegen (1971), der in seinem grundlegenden Werk den Entropie-Ansatz in die ökonomische Theorie integriert hat. Faber et al (1983) haben, aufbauend auf diesem Ansatz, eine "naturwissenschaftlich ökonomische Untersuchung zum Umweltschutz und Rohstoffverbrauch" vorgelegt.

9) Ein öffentliches Gut ist durch zwei Merkmale gekennzeichnet: (i) Nichtrivalität bei der Nutzung, (ii) Nichtausschließbarkeit von potentiellen Nutzern [vgl. Siebert $(1978,9)]$. Zur Begriffsvielfalt des Terminus "öffentliches Gut" und eines Definitionsversuches vgl. Blümel et al (1984). Die Umweltqualităt ist damit ein öffentliches Konsumgut: "Ein technischer Ausschluß, so er möglich wäre, ist gesellschaftspolitisch nicht erwünscht, und das Gut kann von allen Individuen genutzt werden" [Siebert $(1978,9)$ |. Eine Modifikation des öffentlichen Gutsaspekts wird durch die von Dales (1968) ausgehende Diskussion zur Privatisierung der Umwelt vorgestellt, die in Beitrăgen von z. B. Montgomery (1972), Bonus (1981) weiterentwickelt wurde.

10) Zur Problematik des Konzepts einer sozialen Wohlfahrtsfunktion vgl. die Diskussion in Kapitel 3, Abschnitt 1.4. 
qualität umkehrbar. Eine andere Problematik der Umwelt als öffentliches Konsumgut untersucht Krutilla (1967): Wird ein Naturbereich zu Produktionsaktivitäten (z. B. Tagebau von Kohle) eingesetzt, dann ist er nicht mehr als Freizeit- und Erholungsgebiet nutzbar. Hier entstehen Irreversibilitäten, die bei der Entscheidung, ob die Produktionsaktivität aufgenommen werden soll, berücksichtigt werden müssen. Krutilla (1967) und Krutilla, Fisher (1975) lehnen ihre Untersuchungen an konkrete Fälle (den Hells-Cañon-Fall bzw. den Fall der White Cloud Peaks in Idaho) an. Fisher, Krutilla, Cicchetti (1972) analysieren mit Hilfe der Kontrolltheorie die optimalen Nutzungsfolgen von Projekten mit Irreversibilitäten im Umweltbereich. Sie zeigen, daß es suboptimal sein kann, die Produktion aufzunehmen, obwohl der Gegenwartswert der Produktionsaktivität positiv ist, da der Umweltnutzen mit einbezogen werden muß und verschiedene, in der Zukunft liegende Startpunkte der Produktion mit ins Kalkül gezogen werden müssen. Als eine solche Irreversibilität kann auch das bei uns auftretende Waldsterben definiert werden. Im folgenden wird jedoch davon ausgegangen, daß die Umweltschäden grundsätzlich reparabel sind; damit sind Irreversibilitäten ausgeschlossen.

Die Umwelt als Schadstoffaufnahmemedium bildet den Hauptuntersuchungsgegenstand der umweltökonomischen Arbeiten. Durch die Produktion und/oder den Konsum fallen Kuppelprodukte an, die weder für den Konsum noch für die Produktion verwertbar sind und als Emissionen an die Umwelt abgegeben werden ${ }^{11}$. In dieser Arbeit werden nur Produktionsexternalitäten ${ }^{12)}$ untersucht.

Im folgenden wird eine Abgrenzung der Literatur vorgenommen. Insbesondere wird dabei auf regionale Umweltallokationsmodelle ${ }^{13)}$ eingegangen, denn ein Ziel dieser Arbeit ist es, interregionale intertemporale Zusammenhänge aufzuzeigen. Eine intertemporale Sichtweise des Pro-

11) Zur Definition der ökologischen Begriffe vgl. Abschnitt 3 dieses Kapitels.

12) Zu Konsumexternalitäten vgl z. B. Plourde (1972), Forster (1973), Mathur (1974).

13) Input-Output-Modelle, die auch als Grundlage empirischer Abschätzungen von Umweltmaßnahmen dienen [vgl. z. B. Conrad (1976)], werden im folgenden nicht betrachtet. Vgl. hierzu die grundlegende Arbeit von Leontief (1970), die Einführung von Ayres (1978, Kapitel 4ff) sowie die Literaturübersicht bei Johnson, Bennett (1981). 
blems ${ }^{14)}$ ist erforderlich, da "low levels of (.) pollution deposited over years can accumulate to cause environmental damage" [Wetstone, Rosencranz $(1982,3)]$. Jedoch wurde die intertemporale Umweltallokation hauptsächlich "in a wonderland of no other decision units" [Nijkamp $(1978,154)]$ untersucht ${ }^{15}$; die direkten interregionalen Auswirkungen der Schadstoffdiffusion wurden nicht in die dynamische Analyse mit einbezogen. Durch einen intertemporalen Modellzusammenhang können die Eigenschaften des Ökosystems mit in Betracht gezogen werden ${ }^{16}$. Deshalb wird im folgenden eine intertemporale Modellanalyse durchgeführt, die beide Hauptpunkte der intertemporalen interregionalen Umweltallokation berücksichtigt: "The first feature concerns the international (interregional) nature of the (..) phenomenon. The second is the dynamic nature of the phenomenon that causes the welfare of future generations to be affected. Thus formulated the policy maker is confronted with an optimization problem that needs to be solved across nations (regions) and through time" [Forster $(1982,3)]$.

In der Literatur [vgl. z. B. Evans (1980) ${ }^{17)}$ werden folgende regionalen Dimensionen des Umweltproblems unterschieden:

(1) Für ein Individuum ist die Umweltqualität seiner Region am wichtigsten. Man kann sich auf den Standpunkt stellen, daß ein Individuum nur an der Umweltqualität seiner Region interessiert ist. Unter diesem Gesichtspunkt ist Umweltpolitik "perforce regional, indeed intraregional, in nature" [Evans $(1980,185)]$. Unter diesem Konzept kann man all diejenigen Modelle zusammenfassen, bei denen keine interregionalen Effekte

14) Vgl. für einen kurzen Überblick über dynamische ökonomische Modelle unter Einbeziehung der Umwelt Ayres (1978, 17-23).

15) Vgl. z. B. d'Arge, Kogiku (1973), Forster (1973), Mäler (1974), Schulze (1974), Weinstein, Zeckhauser (1974), Wright (1974), Vogt (1981), Snower (1982). Vgl. aber Gebauer (1982, 1984), die die Probleme der intertemporalen Umweltallokation in einem Zwei-Regionen-System beleuchtet.

16) Als Beispiel kann hier auf die Auswirkungen des sauren Regens verwiesen werden: In Norwegen, wo die Böden wenig kalkhaltig sind, sind die Auswirkungen von Schwefelablagerungen schon zwanzig Jahre spürbar, während in der BR Deutschland die Auswirkungen des sauren Regens erst Ende der siebziger, Anfang der achtziger Jahre in ihrem ganzen Ausmaß erkannt wurden.

17) Vgl. für eine allgemeine Diskussion der regionalen Umweltproblematik auch Siebert (1980a und b). 
auftreten, d. h. bei denen eine Region bzw. Ökonomie als geschlossenes System betrachtet wird ${ }^{18)}$.

(2) Die Schadstoffe, die in einer Region emittiert werden, können die Umweltqualität einer anderen Region mitbestimmen, d. h. Umweltpolitik hat interregionale Effekte, die berücksichtigt werden müssen. Die Auswirkungen können folgendermaßen unterteilt werden:

(a) Indirekte Auswirkungen.

Ist eine Ökonomie über Handelsbeziehungen am Weltmarkt beteiligt, dann ergeben sich durch die Einflüsse der Handelsbeziehungen auf die Produktions- und/oder Konsumstruktur Auswirkungen auf die Umweltpolitik in dieser Ökonomie und umgekehrt. Hier ist die zu untersuchende Ökonomie ein offenes System, in dem auch Einflüsse berücksichtigt werden müssen, die durch andere Marktteilnehmer in die Ökonomie hineingetragen werden, ohne daß diese darauf Einfluß nehmen kann. Vgl. hierzu die Arbeiten von Pethig (1976), Siebert (1977, 1978, 1979a, 1981, 1984) Siebert, Eichberger et al (1980), Gronych (1980), Walter (1975), Baumol, Oates (1975), Asako (1979). Nur Asako berücksichtigt die Schadstoffakkumulation, hat also, neben einer statischen, auch eine dynamische Modellstruktur gewählt.

Darunter können auch die Auswirkungen der Umweltpolitik auf die Standortwahl emissionsintensiver Unternehmen gefaßt werden. Es wird also nicht die Mobilität der Schadstoffe bzw. der Güter und Faktoren betrachtet, sondern es wird diskutiert, inwieweit die Aktivitäten, die Schäden verursachen, zwischen den Regionen wandern ${ }^{19}$. Auf diesen Teilaspekt wird im folgenden nicht eingegangen.

(b) Direkte Auswirkungen

Hier stehen die direkten, durch Diffusionsvorgänge hervorgerufenen interregionalen Auswirkungen im Vordergrund. Dabei ist zu unterscheiden, ob die Diffusion von Schadstoffen nur in eine Richtung geht (UnterliegerOberlieger-Problematik), gegenseitig ist oder das verschmutzte Ökosystem eine Allmende-Ressource ist. Die explizite Einbeziehung von Schadstofftransport über Regionen- und Ländergrenzen hinweg wurde bis jetzt

18) Vgl. z. B. Siebert (1978), d'Arge, Kogiku (1973), Mäler (1974), Vogt (1981).

19) Vgl. hierzu z. B. Kneese, Williams (1980) und Evans (1978). 
vor allem in statischen Modellen vorgenommen ${ }^{2021)}$. Van Zele (1978) benutzt einfache spieltheoretische statische Modelle sowohl kooperativer als auch nicht-kooperativer Art, um das Umweltallokationsproblem zwischen Ländern zu lösen. Førsund (1980) untersucht die Frage, wie Investitionen auf zwei Regionen einer Ökonomie von einem zentralen Planer auf optimale Weise zugeordnet werden unter Berücksichtigung der zweiseitigen Schadstoffdiffusion. Førsund hat - in Anlehnung an Rahman (1963, 1966), Intriligator (1964), Takayama (1974) und Pitchford (1977) - ein intertemporales Zwei-Regionen-Modell entwickelt, bei dem die Emissionen bzw. Immissionen in die zu maximierende Wohlfahrtsfunktion mit aufgenommen werden. Hier spielt der Umweltaspekt nur eine Nebenrolle. Ist das Umweltmedium, in das die Schadstoffe eingeleitet werden, von mehreren Regionen bzw. Ländern nutzbar, dann hat dieses Umweltmedium die "common property"-Eigenschaft [vgl. Pethig $(1982,58)]^{22}$. Pethig (1982) und Gladwin et al (1982) diskutieren als Beispiel für eine common property Ressource die Stratosphäre, die als Aufnahmemedium von Fluorchlorkohlenstoff dient, wobei sich Pethig spieltheoretischer Modelle bedient. Forster (1978) analysiert in einem dynamischen Kontext ein Staatengebilde aus zwei souveränen Staaten, die ein gemeinsames Ökosystem verschmutzen. Arnold (1984) untersucht, ebenfalls mit spieltheoretischen Methoden, die Verschmutzung eines gemeinsamen Umweltmediums durch zwei Länder in einem statischen Kontext. Als Ansatzpunkt verwendet er die Theorie international öffentlicher Güter ${ }^{23)}$ und zeigt, daß eine

20) Vgl. z. B. Smets (1974), Siebert $(1975,1978,1979$ a, 1981, 1984), Dudenhöffer (1979, 1983), OECD (1974), verschiedene Beiträge in dem grundlegenden Werk zur regionalen Umweltallokation Siebert, Walter, Zimmermann (1980).

21) Vgl. jedoch Gebauer (1982), die in einem intertemporalen Modellrahmen zeigt, daß ein Steady-State existiert, wenn zwei Regionen sich gegenseitig Schadstoffe einleiten; und Loehman, Whinston (1970), die die Oberlieger-Unterlieger-Problematik bezogen auf zwei Firmen in einem dynamischen Kontext untersuchen. Vgl auch den Kommentar von Forster, Logan (1973).

22) Vgl. zu der intertemporalen Nutzung erneuerbarer Allmende-Ressourcen Siebert (1983b), der einen systematischen Überblick gibt; vgl. z. B. auch Munro (1979) und Pethig (1982, 1984a). Für nicht erneuerbare Allmende-Ressourcen vgl. z. B. die Literaturübersicht von Pethig (1984b). Vgl. auch die in den Papers and Proceedings des American Economic Review (1982) zusammengefaßten Artikel von Nordhaus, Bailey, Lave, Olson, Dowing und Kates.

23) Vgl. z. B. Olson, Zeckhauser (1967, 1970), Breton (1970), Pauly (1970), Sandler, Culyer (1982a und b). 
Spezialisierung bei der Produktion des öffentlichen und des privaten Gutes aufgrund komparativer Kostenvorteile für beide Länder vorteilhaft ist.

In dieser Arbeit werden die direkten und indirekten Auswirkungen der regionalen Umweltallokation miteinander verknüpft. Bei den direkten Auswirkungen beschränken wir uns auf die Unterlieger-Oberlieger-Problematik, die ausführlich in Kapitel 4 dargestellt wird. Hierbei werden drei unterschiedliche institutionelle Arrangements diskutiert: Autonome regionale Umweltbehörden, eine zentrale Umweltbehörde und beschränkt autonome Umweltbehörden. Die indirekten Auswirkungen über Handelsbeziehungen - die betrachtete Zwei-Regionen-Ökonomie ist ein kleines Land - werden in Kapitel 5 untersucht. In Kapitel 6 wird eine Integration dieser beiden interregionalen Auswirkungen vorgenommen, um so das Beziehungsgefüge zwischen zwei Regionen in einem intertemporalen $\mathrm{Zu}$ sammenhang mit Umweltpolitik darzustellen.

(3) Individuen können an der Umweltqualität anderer Regionen interessiert sein, weil sie diese Regionen besuchen wollen (z.B. Urlaub) ${ }^{24)}$. Dieser Aspekt wird nicht behandelt.

Unter diesem Punkt kann auch, bezogen auf Konsumenten, der unter dem Schlagwort "voting with one's feet" bekanntgewordene Vorschlag Tiebout's (1956) subsumiert werden. Die These Tiebout's ist, daß das Allokationsproblem lokaler öffentlicher Güter effizient dezentral gelöst werden kann. Liegen in unterschiedlichen Regionen verschiedene Umweltqualitäten vor, dann würden, Tiebout folgend, die Konsumenten in die Region wandern, in der ihre Präferenzen maximiert werden. Ein Gleichgewicht ist dann erreicht, wenn kein Konsument mehr einen Anreiz hat, die Regi-

24) Vgl. z. B. Frey (1980), Potier (1980). 
on zu wechseln. In neueren Beiträgen ${ }^{25)}$ wird gezeigt, daß effiziente "Tiebout-Gleichgewichte" nur unter sehr restriktiven Annahmen existieren.

Dem Tiebout-Ansatz der Konsumentenwanderung wird nicht gefolgt, obwohl die Faktoren ebenfalls als mobil vorausgesetzt werden (Kapitel 5 und 6). Es ist hier eine Modellform gewählt, bei der einer Region jeweils eine bestimmte Menge an Faktoren zur Verfügung steht, die zu jedem Zeitpunkt in den Produktionsprozeß eingesetzt werden kann und/oder für deren Gegenwert Konsumgüter gekauft werden. Es wird also ein "Flow"Konzept der Faktormobilität betrachtet, das den Faktorbestand für jeden Zeitpunkt unverändert läßt, während beim Tiebout-Ansatz die Mobilität auf den Faktorbestand bezogen ist.

25) Einen Überblick über die Literatur zum Tiebout-Theorem gibt Pestieau (1983). Stiglitz (1977) faßt die Theorie lokaler öffentlicher Güter in einem formalen Ansatz zusammen. Mit der Existenz und der Pareto-Optimalität von Wanderungsgleichgewichten beschäftigen sich Westhoff (1977), Starrett (1978), Wooders $(1979,1980)$, Bewley (1981), Stahl, Varaiya (1983), von dem Hagen (1983). Eng mit den Problemen lokaler öffentlicher Güter ist die Theorie von Club-Gütern verbunden [vgl. Buchanan (1965)]. Einen Überblick über die neuere Literatur zu Club-Gütern und lokalen öffentlichen Gütern geben Sandler, Tschirhart (1980). Dudenhöffer, Gebauer (1982) diskutieren das Tiebout-Theorem unter besonderer Berücksichtigung unterschiedlicher Steuerarten zur Finanzierung des lokalen öffentlichen Gutes und der optimalen Gemeindegröße. 


\section{3. ÖKOLOGISCHE GRUNDLAGEN}

In diesem Abschnitt werden die Grundlagen für die in den folgenden Kapiteln relevanten ökologischen Tatbestände herausgearbeitet. Das Umweltproblem hat neben den ökonomischen vor allem auch chemische, geophysikalische und biologische Aspekte ${ }^{26}$. Die Gesamtheit aller dieser Dimensionen des Umweltproblems kann jedoch innerhalb dieser Arbeit nicht dargestellt werden, sondern wir wollen uns auf diejenigen Tatbestände beschränken, die für die im folgenden zu entwickelnden Modelle von Bedeutung sind. In Abschnitt 3.1 wird eine Definition des Ökosystems gegeben, und die für die Analyse wichtigen Begriffe werden geklärt. In Abschnitt 3.2 werden die Grundzüge der Diffusion von Schadstoffen in einem Umweltmedium dargestellt.

\subsection{DAS ÖKOSYSTEM ${ }^{27)}$}

Ein Ökosystem ist "ein Wirkungsgefüge von Lebewesen und deren anorganischer Umwelt, das zwar offen, aber bis zu einem gewissen Grade zur Selbstregulierung befähigt ist" [Ellenberg $(1973,1)]$. Dieses Wirkungsgefüge besteht aus

- dem Biotop: Darunter fallen die unbelebten (abiotischen) Elemente eines räumlich abgrenzbaren Gebietes wie Klima, Oberflächengestalt, bauliche und technische Anlagen, Boden Wasser, Luft etc; und

- der Biozönose: Den im Biotop befindlichen Lebewesen wie Pflanzen, Tiere, Mikroorganismen und Menschen.

Zwischen dem Biotop und der Biozönose bestehen vielfältige Zusammenhänge, die sich z. B. durch Energieflüsse, Nahrungsketten und Stoffkreisläufe ausdrücken [vgl. Schaubild 2.1]. Die Gesamtheit aller Ökosysteme ist die Ökosphäre (Biosphäre), die sich in Süßwasser-, Meereswasser-, Land- und Land/Wasser-Ökosysteme unterteilen läßt.

26) Neben den oben aufgeführten sind natürlich auch rechtliche, philosophische, medizinische und andere Aspekte relevant. Darauf năher einzugehen, würde jedoch den Rahmen dieser Arbeit sprengen. Allein für die schwierigen juristischen Tatbestände der grenzüberschreitenden Verschmutzung gibt es eine Fülle von Literatur, vgl. z. B. OECD (1977), Mingst (1982), Ercman (1977) und die dort angegebene Literatur. Eine "Philosophische Studie zum Ökologieproblem der Industriegesellschaft" wurde von Hassenpflug (1980) vorgelegt.

27) Vgl. Hartkopf, Bohne (1983b, 22); vgl. auch Clark, Jones, Holling (1979), die eine Übersicht über die Őkosystemforschung geben. 
In schematischer Form ist ein Ökosystem in Schaubild 2.1 dargestellt.

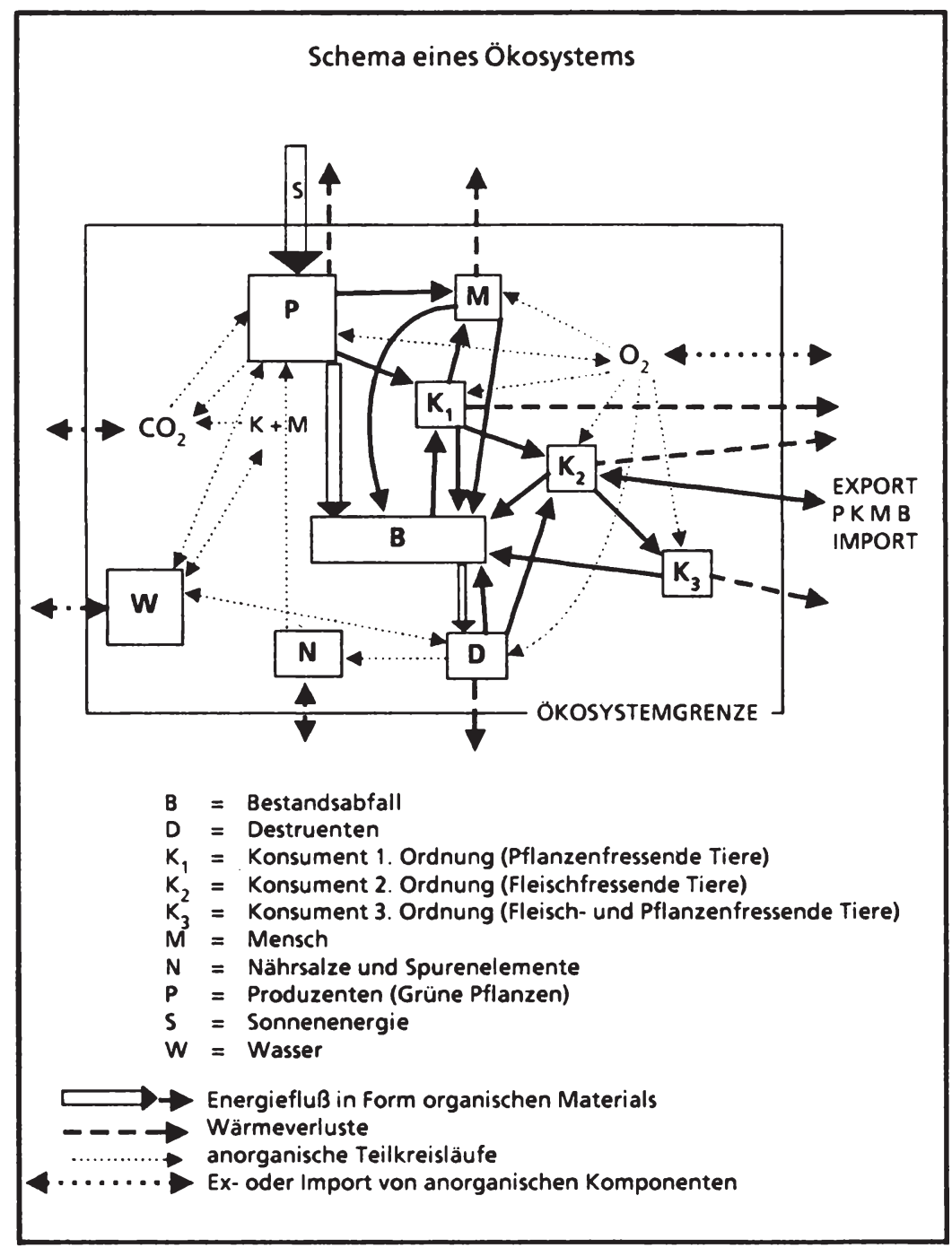

Schaubild 2.1 : Schema eines Ökosystems ${ }^{28)}$

28) Quelle: SRU (1974, 295). 
Eine wichtige Eigenschaft des Ökosystems ist die begrenzte Selbstregulierung oder Assimilationsfähigkeit. Diese ist "abhängig von den abiotischen Standortfaktoren (klimatische Faktoren, geologische Verhältnisse; Disposition im Sinne von Ellenberg (1973)). Mit anderen Worten, ein Ökosystemtyp kann regional verschiedene Reaktionen auf gleiche Belastung zeigen" [SRU (1974, 263)]. Der Schutz vor "Belästigungen" eines Ökosystems ist eines der Ziele der Umweltpolitik. Dieses Ziel ist ein Unterziel der Hauptaufgabe des Umweltschutzes, des Schutzes der Menschen vor Schaden [SRU $(1978,17)]$.

Belästigungen oder Lasten, die ein Umweltsystem zu tragen hat, sind chemische und/oder physikalische Faktoren, die durch menschliche Tätigkeiten (Produktion, Konsum) in das System gelangen, also das natürliche Vorkommen überschreiten. "Als "Schadstoffe" werden solche in der Umwelt vorkommenden Stoffe bezeichnet, die das Potential haben, auf den Menschen, auf andere Lebewesen, auf Ökosysteme oder auch auf Sachgüter schädlich zu wirken" [SRU $(1978,18)]$.

Hier an dieser Stelle sollen die Termini erklärt werden, die im folgenden die Belastungen des Ökosystems ausdrücken. Durch Produktion und Konsum können Kuppelprodukte ${ }^{29)}$ entstehen, die weder für die Produktion ${ }^{301}$ noch für den Konsum weiter verwertbar sind. "(...) those materials and energy flows (.) have no value in existing markets or a value less than their costs of collection, transportation and use in the same or another activity" [Bower, Basta $(1982,2)]$. Die so entstehenden Kuppelprodukte werden im folgenden Emissionen genannt. Emissionen unterliegen nach ihrer Abgabe an die Umwelt diffusiven und transformativen Einflüssen ${ }^{30}$. Durch diese Umwandlungen und durch Akkumulation entstehen Immissionen. Immissionen bezeichnen also die Menge an Schadstoffen, die sich in einem bestimmten Zeitpunkt an einer bestimmten Stelle eines Umweltmediums befinden.

29) Vgl. Siebert $(1978,11)$.

30) In der vorliegenden Arbeit werden nur aus der Produktion entstehende Umwelteffekte behandelt. Vgl. aber Mathur (1974).

31) Vgl. die Ausführungen in Abschnitt 3.2 dieses Kapitels. 
Innerhalb eines ökonomischen Modells ist es nicht möglich, die realen komplexen Zusammenhänge eines Ökosystems - wie in Schaubild 2.1 vereinfacht dargestellt - abzubilden. Deshalb werden im folgenden eine Reihe vereinfachender Annahmen bezüglich des Ökosystems gemacht [vgl. auch Vogt $(1981,15)$ ]:

- es gibt nur eine Art von Emissionen, ihre Menge ist homogen;

- zwischen den einzelnen Umweltmedien wird nicht unterschieden;

- der Immissionsbestand einer Region ist eine homogene Menge;

- die Schäden, die durch die Immissionen auftreten, werden in einer Wohlfahrtsfunktion quantifiziert.

\subsection{DIFFUSION UND ADVEKTION}

\subsubsection{Grundzüge der Diffusionstheorie}

Der Begriff der Diffusion beschreibt ganz allgemein die Tendenz von einer Anzahl von Partikeln, die ursprünglich in einem Raumpunkt konzentriert sind, sich mit der Zeit auszubreiten, indem sie eine immer größere Fläche rund um den Ursprungspunkt bedecken ${ }^{32}$. Darunter sollen nur nicht zielgerichtete Partikelbewegungen fallen, da unter Diffusion nur das Phänomen verstanden wird, bei dem sich eine Partikelgruppe als ganzes gemäß der irregulären (zufälligen) Bewegung jedes Partikels ausbreitet ${ }^{33}$.

Die klassische Diffusionstheorie wurde vor mehr als hundert Jahren von dem Physiologen A. Fick (1855) begründet. Er hat die Diffusion auf eine quantitative Basis gestellt, indem er die Fourier'sche Gleichung der Wärmeleitung übernahm. Die mathematische Theorie der Diffusion in isotropen $^{34)}$ Substanzen basiert deshalb auf der Hypothese, daß die Transferrate einer diffusierenden Substanz durch eine Einheit Fläche in $x$-Richtung in einer Zeiteinheit, d. h. der Fluß $J_{x}$, proportional ist zum Konzentrationsgradienten der Masse:

32) Vgl. Okubo $(1980,8)$

33) Vgl. Okubo $(1980,8)$, Crank $(1979,1-2)$. Auch die folgenden Ausführungen basieren auf diesen Arbeiten.

34) Nach allen Richtungen hin gleiche Eigenschaften aufweisend (physikalisch). 


$$
J_{x}=-D \frac{\partial C}{\partial x}
$$

wobei $C$ die Konzentration der diffusierenden Substanz und $D$ der Diffusionskoeffizient ist. Gleichung (2.1) wird auch erstes Fick'sches Gesetz genannt.

Die Fick'sche Diffusionsgleichung oder das zweite Fick'sche Gesetz ist

$$
\frac{\partial C}{\partial t}=-\frac{\partial J_{x}}{\partial x}=\frac{\partial}{\partial x}\left(D \frac{\partial C}{\partial x}\right)
$$

d. h. die Konzentrationsänderung in der Zeit ist gleich der absoluten Änderung von $J_{x}$ in Richtung $x$.

Fick's Gesetze gelten für die molekulare Diffusion, bei der das betrachtete Medium laminar ist. Dies gilt jedoch nicht für die Atmosphäre und die Ozeane. Dort ist die Bewegung nicht in Schichten geordnet, sondern es herrscht turbulente Bewegung. Unter Turbulenz wird hier eine Art zufälliger Bewegung verstanden, die aus vielen überlagerten Wirbeln besteht, die von Raum und Zeit abhängig sind. Die Bewegung von Partikeln in einem Umweltmedium wird von der Turbulenz beeinflußt und ist deshalb zufällig. Ist in der Bewegung Zufälligkeit beinhaltet, wird sie von Diffusion begleitet. Wir nennen die Diffusion, die von Umweltströmungen herrührt, turbulente Diffusion oder Dispersion, um sie von der molekularen Diffusion zu unterscheiden, die von der zufälligen molekularen Bewegung abhängt. Turbulente Diffusion ist von Natur großräumig.

Wird die Diffusion ökologischer Systeme betrachtet, erscheint eine andere Art von Diffusion zusätzlich zur turbulenten Diffusion, die Biodiffusion, die von zufälligen Bewegungen der Organismen selbst mit abhängt. Diese Art der Diffusion wird aktiv genannt, da die Partikel eine Eigenbewegung haben. Im Gegensatz dazu wird die Diffusion von Schadstoffen passiv genannt, da sie sich nur aufgrund von Bewegungen des sie enthaltenden $\mathrm{Me}$ diums verteilen. 
Wie in Abbildung 2.2 dargestellt, ist das Diffusionsproblem sehr vielschichtig und wird von Physikern, Chemikern, Biologen, Meteorologen, Geologen u. a. untersucht, je nachdem, welche Substanz bzw. Spezies diffusiert.

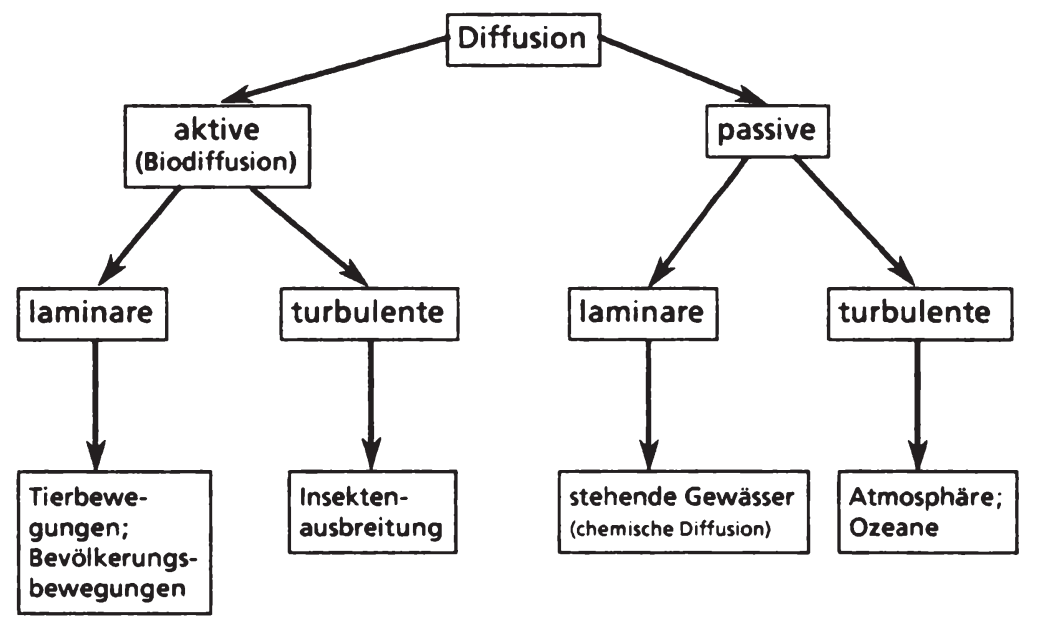

Schaubild 2.2: Ausprägungen der Diffusion

Wir wollen uns im folgenden mit der passiven, turbulenten Diffusion beschäftigen. Dieser rechte Zweig in Abbildung 2.2 bezieht sich auf die Umweltmedien Luft und Wasser, wobei wir uns bei der Erklärung der Diffusionsmodelle i. a. auf das Umweltmedium Luft ${ }^{35)}$ beschränken.

\subsubsection{Diffusions-bzw. Dispersionsmodelle ${ }^{36)}$}

Die grundlegende Frage, die allen Dispersionsmodellen zugrunde liegt, ist: "What are, within a specified region, the ambient concentrations at

35) Vgl. zur Luftverschmutzung das von Stern (1976) herausgegebene grunglegende Werk in fünf Bånden.

36) Eine einfache Einfuhrung geben Waite, Freeman (1977). 
arbitrary times and positions of a certain (.) material, due to emissions elsewhere at previous times?" [Van Dop $(1983,52)$ ]. Diese Frage kann grundlegend mit drei analytischen Konzepten angegangen werden: (i) Physikalische Modelle, (ii) Masse- und Energieerhaltungsmodelle, (iii) Statistische Modelle [vgl. Basta, Moreau (1982, 37)].

Drei Charakteristika sind für alle Natürliche-System-Modelle (NSM) zu definieren: (i) die zeitlichen Änderungsraten: wie verändern sich die Variablen eines NSM über die Zeit; (ii) die durchschnittliche Zeit: über welche Zeiteinheit werden die Variablen eines NSM gemittelt; und (iii) die räumliche Dimension: wieviele Dimensionen sollen in einem NSM enthalten sein und in wieviele Segmente soll das NSM aufgeteilt werden [vgl. Basta, Moreau $(1982,39)]^{37)}$

\subsubsection{Der Energie-und Masseerhaltungsansatz}

Im folgenden werden die Grundzüge von Modellen vorgestellt, die sich auf das Gesetz der Erhaltung der Masse beziehen, da diese später im Modellteil in die Analyse mit einbezogen werden. Diese Modelle versuchen explizit, den Transport und die Umwandlung von Material in einem natürlichen System abzubilden [vgl. Basta, Moreau $(1982,38)]$.

Die Masse- und Energieerhaltungsmodelle werden in der Analyse der Natürlichen Umweltsysteme (Wasser, Luft) ${ }^{38)}$ am häufigsten angewandt. Es wird davon ausgegangen, daß die Erde ein geschlossenes System ist, so da $\beta$ keine Masse- und Energievernichtung vorkommen kann, sondern es kann nur eine Umwandlung in einen anderen Aggregatzustand geben.

37) Eine weitere Unterscheidung der NSM ergibt sich aus den Annahmen über das Verhalten der Modellvariablen in der Zeit: Steady-State-Modelle (die Variablen ändern sich in der gegebenen Zeitperiode nicht) und Nichtsteady-State-Modelle (alle Variablen ändern sich in der gegebenen Zeitperiode). Auf diese Unterscheidung wird im folgenden jedoch nicht weiter eingegangen.

38) Vgl. Basta, Bower (eds.) (1982), wo ein Überblick über die angewandten Methoden für die Analyse natürlicher Systeme gegeben wird. Die folgenden Ausführungen stützen sich z. T. auf Beiträge dieses Buches, insbesondere auf Basta, Moreau (1982, 23-120), sowie Muschett (1982, 389-546). Weiterhin wird auf Dobbins (1979, Kapitel 9-11) bezug genommen. 
Analysiert man natürliche Systeme unter Berücksichtigung des Masseund Energieerhaltungsgesetzes, wird das System in Raumeinheiten aufgeteilt und man untersucht die Bewegung von Massen- und Energieströmen in der Zeit von Raumeinheit zu Raumeinheit, indem man die Masse und Energiegleichgewichtsbedingungen betrachtet.

Mathematisch kann das Masseerhaltungsgesetz folgendermaßen ausgedrückt werden:

$$
\frac{d C}{d t}=\sum_{i} p_{i}-\sum_{j} q_{j}+\sum_{k}\left( \pm s_{k}\right)
$$

wobei $C$ wiederum die Konzentration einer Substanz innerhalb eines Raumes ausdrückt, $p$ die Zuströme von außerhalb des Raumes, $q$ die Abflüsse nach außerhalb des Raumes und $s$ die innerhalb eines Raumes produzierten bzw. abgebauten Mengen der Substanz ausdrückt.

Dieses so beschriebene Modell ist das einfachste, das angewandt wird. Um realitätsnähere Aussagen zu bekommen, wird Gleichung (2.3) so modifiziert, daß tatsächliche Abläufe - oder Aspekte davon - miteinbezogen werden können. Im folgenden werden Modelle beschrieben, die auf den obigen Grundlagen basieren. Im einzelnen wird beschrieben, wie Advektion und Diffusion integriert werden.

Diffusion wurde schon in Abschnitt 3.2.1 definiert. Unter Advektion versteht man den Prozeß, durch den Substanzen durch den Raum transportiert werden durch die Bewegung des Fließmediums, in dem die Substanz enthalten bzw. aufgelöst ist.

Zur Ableitung der grundlegenden Gleichung der Dispersion wird folgendes Gedankenexperiment gemacht. Wir betrachten eine Mixtur, die aus zwei Komponenten $A$ und $B$ besteht. Dabei wird die molekulare Diffusion untersucht, die auch auftritt, wenn keine Druck- und/oder Temperaturunterschiede bestehen, wenn keine Gravitations- oder andere Körperkräfte 
wirken oder eine Fließbewegung vorhanden ist. Dann gilt Fick's erstes Gesetz:

$$
J_{A}=J_{x_{A}}+J_{y_{A}}+J_{z_{A}}=-\rho D_{A B}\left[\frac{\partial}{\partial x}\left(\frac{C_{A}}{\rho}\right)+\frac{\partial}{\partial y}\left(\frac{C_{A}}{\rho}\right)+\frac{\partial}{\partial z}\left(\frac{C_{A}}{\rho}\right)\right]^{399}
$$

wobei $D_{A B}$ der binäre Diffusionskoeffizient ist, der die Massediffusivität der Substanz $A$ in Substanz $B$ ausdrückt und $\rho$ die Massendichte ist ( $\rho=$ $C_{A}+C_{B}$ ). Das Massenerhaltungsprinzip für die Substanz $A$ kann folgendermaßen ausgedrückt werden:

$$
\iiint_{V} \frac{\partial C_{A}}{\partial t} d V=-\iint_{S}\left(C_{A} q_{A}\right) d S+\iiint_{V} S_{A} d V
$$

(iii)

wobei $q_{A}$ die Eigengeschwindigkeit der Substanz $A$ ist ${ }^{411}$.

Die drei Terme repräsentieren

(i) Die Nettorate der Akkumulation der Substanz A im Kontrollraum

(ii) Die Durchströmungsrate der Substanz $A$ durch die Grenzen der Kontrollfläche

(iii) Die Gesamtproduktion von $\boldsymbol{A}$ im Kontrollraum als Ergebnis einer volumetrischen chemischen Produktionsrate $S_{A}$, die die Masseeinheiten pro Raumeinheit pro Zeiteinheit erzeugt.

39) $x$ und $y$ geben die horizontalen Richtungen an, während $z$ die vertikale Richtung symbolisiert; dies gilt auch für die weiteren Ausführungen in diesem Kapitel. In Schaubild 2.3 sind die Richtungen dargestellt.

$x$ gibt dabei immer die Hauptbewegungsrichtung an

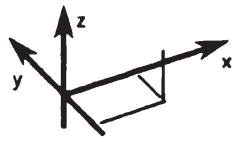

Schaubild 2:3 (Windrichtung, Flußströmung, Meeresstrōmung).

40) $V=$ Volumen $\Rightarrow d V=d x d y d z$.

$S=$ Surface (Fläche) $\Rightarrow d S=d y d z$,

wobei die Fläche normal zur $x$-Achse ist.

41) $q_{A}$ bzw. $q$ ist ein Vektor, der die Geschwindigkeiten in die verschiedenen Richtungen des Raumes beinhaltet: $q=\left(u_{x}, u_{y}, u_{z}\right)$, wobei $u_{x}$ die Geschwindigkeit in Richtung $x$ etc. darstellt. 
Das Flächenintegral in Gleichung (2.5) wird durch das Gauß-Theorem ${ }^{42)}$ in ein Volumenintegral umgewandelt und alle Terme können unter ein Integral geschrieben werden:

$$
\iiint_{V}\left|\frac{\partial C_{A}}{\partial t}+\nabla\left(C_{A} q_{A}\right)-S_{A}\right| d V=0
$$

Da das Integral für jedes geeignet gewählte $V$ verschwindet und der Integrand stetig ist, folgt

$$
\frac{\partial C_{A}}{\partial t}+\nabla\left(C_{A} q_{A}\right)=S_{A}
$$

Dies ist die Stetigkeitsgleichung für Substanz A, die die Änderung der Konzentration dieser Substanz in jedem Raumpunkt ausdrückt, wenn Bewegung und chemische Reaktion vorhanden sind.

Betrachten wir jetzt statt $q_{A}$ eine Durchschnittsgeschwindigkeit $q$. Es gilt $J_{A}=C_{A}\left(q_{A}-q\right)$; daraus folgt nach einsetzen und einigen Umformungen unter der Annahme, daß die Dichte der Mixtur konstant ist ${ }^{43)}$

$$
\begin{aligned}
\frac{\partial C_{A}}{\partial t}= & -u_{x} \frac{\partial C_{A}}{\partial x}-u_{x} \frac{\partial C_{A}}{\partial x}-u_{x} \frac{\partial C_{A}}{\partial x} \\
& +\frac{\partial}{\partial x}\left(D_{A B} \frac{\partial C_{A}}{\partial x}\right)+\frac{\partial}{\partial y}\left(D_{A B} \frac{\partial C_{A}}{\partial y}\right)+\frac{\partial}{\partial z}\left(D_{A B} \frac{\partial C_{A}}{\partial z}\right) \pm S
\end{aligned}
$$

Im allgemeinen ist der molekulare Diffusionskoeffizient $D_{A B}$ eine Funktion der Konzentration, die ihrererseits wieder von der Diffusion beeinflußt wird. Ist die Substanz $A$ eine minore Komponente in einem Gasgemisch und die Temperaturschwankungen sind gering, ist der Diffusionskoeffizient konstant und wird mit $D_{A}$ bezeichnet. Wir erhalten

$$
\frac{\partial C_{A}}{\partial t}=-u_{x} \frac{\partial C_{A}}{\partial x}-u_{x} \frac{\partial C_{A}}{\partial x}-u_{x} \frac{\partial C_{A}}{\partial x}+D_{A}\left(\frac{\partial^{2} C_{A}}{\partial x^{2}}+\frac{\partial^{2} C_{A}}{\partial y^{2}}+\frac{\partial^{2} C_{A}}{\partial z^{2}}\right) \pm S
$$

42) Vgl. z. B. Courant (1972, 345 - 350).

43) d. h. $\nabla q=0$. 
Diese Gleichung drückt die Erhaltung jeder Substanz in einer sich bewegenden Strömungsmischung aus. Sie setzt sich zusammen aus der Stetigkeitsgleichung, der Fick'schen Diffusion und chemischen Reaktionen.

Sei die zeitdurchschnittliche Konzentration einer bestimmten Substanz in einem bestimmten Raumpunkt

$$
c=\frac{1}{\Delta \theta} \int_{t-\frac{\Delta \theta}{2}}^{t+\frac{\Delta \theta}{2}} C(t) d t
$$

Dann gilt $C=c+C^{\prime}$, wobei $C^{\prime}$ die zufällige Abweichung vom Mittelwert ist und somit eine Zufallsvariable. Für die Geschwindigkeiten wird eine analoge Transformation vorgenommen. Nach einsetzen in Gleichung (2.7) und einigen Umformungen erhält man

$$
\begin{aligned}
\frac{\partial c}{\partial t}= & -u_{x} \frac{\partial c}{\partial x}-u_{y} \frac{\partial c}{\partial y}-u_{z} \frac{\partial c}{\partial z}+D\left[\frac{\partial^{2} c}{\partial x^{2}}+\frac{\partial^{2} c}{\partial y^{2}}+\frac{\partial^{2} c}{\partial z^{2}}\right] \\
& +\left[\frac{\partial\left(-u_{x}^{\prime} C^{\prime}\right)^{*}}{\partial x}+\frac{\partial\left(-u_{y}^{\prime} C^{\prime}\right)^{*}}{\partial y}+\frac{\partial\left(-u_{z}^{\prime} C^{\prime}\right)^{*}}{\partial z}\right] \pm s
\end{aligned}
$$

wobei die Reaktionsrate $s$ ein in geeigneter Weise umgewandelter Zeitdurchschnitt von $S$ ist. Gleichung (2.7) hat gegenüber (2.6) drei neue Terme $\left(-u_{x}^{\prime} C^{\prime}\right)^{*},\left(-u_{y}^{\prime} C^{\prime}\right)^{*},\left(-u_{z}^{\prime} C^{\prime}\right)^{*}$, die die Korrelation zwischen der Geschwindigkeit und der Konzentration ausdrücken. Sie repräsentieren eine Art von Diffusionsprozeß, der aus der Turbulenz entsteht und werden Reynolds-Dispersions-Terme genannt. Gleichung (2.6) ist für konstantes $D_{A}$ lösbar, während Gleichung (2.7) nicht nur die Änderung der mittleren Konzentration enthält, sondern zusätzlich drei unbekannte Ausdrücke, über die keine Information vorliegt. Ohne zusätzliche Information kann die Gleichung für die turbulente Dispersion nicht gelöst werden ${ }^{44)}$.

Aus Beobachtungen folgt, daß die Reynolds-Dispersions-Terme um das $10^{5}$-fache größer sind als die molekulare Diffusionsterme, so da $\beta$ diese vernachlässigt werden können:

44) Dobbins $(1979,209)$ nennt dies das "closure problem of turbulence". 


$$
\frac{\partial c}{\partial t}=-u_{x} \frac{\partial c}{\partial x}-u_{y} \frac{\partial c}{\partial y}-u_{z} \frac{\partial c}{\partial z}+\left[\frac{\partial\left(-u_{x} C^{\prime \prime}\right)^{*}}{\partial x}+\frac{\partial\left(-u_{y}^{\prime} C^{\prime}\right)^{*}}{\partial y}+\frac{\left.\partial-u_{z}^{\prime} C^{\prime}\right)^{*}}{\partial z}\right] \pm s
$$

Damit ist jedoch das "closure problem of turbulence" nicht behoben. Im folgenden wollen wir uns der Lösung dieses Problems zuwenden.

Der turbulente Massetransfer kann durch die Gradienten-Transport-Hypothese folgendermaßen ausgedrückt werden:

$$
J_{i z}^{T}=-\left(-u_{z}^{\prime} C_{i}^{\prime}\right)^{*}=-\rho K_{z z} \frac{d}{d z}\left(\frac{c_{i}}{\rho}\right)
$$

wobei $J^{T}{ }_{i z}$ die durchschnittliche Strömung in $z$-Richtung der $i$-ten Komponenten pro Flächeneinheit ist, die durch das turbulente Strömungsfeld verursacht wird; $K_{z z}$ wird turbulente Diffusivität genannt. Unter der Annahme, daß o konstant ist, können für die Reynolds-Dispersions-Terme folgende Ausdrücke geschrieben werden:

$$
\left(-u_{x}^{\prime} C_{i}^{\prime}\right)^{*}=K_{x x} \frac{\partial c_{i}}{\partial x},\left(-u_{y}^{\prime} C_{i}^{\prime}\right)^{*}=K_{y y} \frac{\partial c_{t}}{\partial y},\left(-u_{z}^{\prime} C_{i}^{\prime}\right)^{*}=K_{z z} \frac{\partial c_{i}}{\partial z},
$$

Das "closure problem" kann gelöst werden, wenn die drei Komponenten der turbulenten Diffusivität $K_{x x}, K_{y y}, K_{z z}$ als Funktionen des Raumes oder der mittleren Fließgeschwindigkeitseigenschaften ausgedrückt werden können ${ }^{45}$. Für die Gleichung der turbulenten Dispersion kann dann geschrieben werden:

$$
\frac{\partial c}{\partial t}=-u_{x} \frac{\partial c}{\partial x}-u_{y} \frac{\partial c}{\partial y}-u_{z} \frac{\partial c}{\partial z}+k_{x} \frac{\partial^{2} c}{\partial x^{2}}+k_{y} \frac{\partial^{2} c}{\partial y^{2}}+k_{z} \frac{\partial^{2} c}{\partial z^{2}}+( \pm s)
$$

Für den eindimensionalen Fall gilt

$$
\frac{\partial c}{\partial t}=-u_{x} \frac{\partial c}{\partial x}+k_{x} \frac{\partial^{2} c}{\partial x^{2}}+( \pm s)
$$

45) Nach Calder (1965) sollten auch die Kreuzterme (z. B. $K_{x y}, K_{x z}$ ) berücksichtigt werden. Da jedoch über sie keine Informationen bezüglich der Richtung und der Größe vorliegen, werden sie Null gesetzt

46) Zur Vereinfachung wurde $K_{x x}$ durch $k_{x}$ ersetzt. Dies gilt auch analog für die beiden anderen Terme. 
Gleichung (2.11) läßt sich anschaulich folgendermaßen in Worten ausdrücken [vgl. Basta, Moreau $(1982,52)]$ :

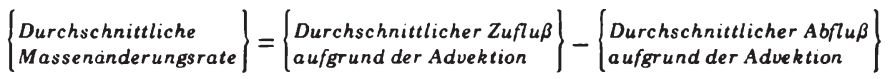

$$
\begin{aligned}
& +\left\{\begin{array}{l}
\text { Durchschnittlicher Zufluß } \\
\text { aufgrund der Diffusion }
\end{array}\right\}-\left\{\begin{array}{l}
\text { Durchschnittlicher Abfluß } \\
\text { aufgrund der Diffusion }
\end{array}\right\} \pm\left\{\begin{array}{l}
\text { Quellen und } \\
\text { abbau }
\end{array}\right\}
\end{aligned}
$$

Gleichung (2.11) ist die grundlegende Gleichung, auf die sich alle Modelle natürlicher Systeme beziehen, die auf dem Gesetz der Erhaltung der Masse beruhen ${ }^{47148)}$, sie wird auch als $K$-Theorie bezeichnet.

\subsubsection{Grundlegende Methoden}

Es gibt zwei grundlegende Methoden, um die Bewegung von Fließmedien aufgrund des Masseerhaltungsgesetzes zu beschreiben:

- Die Lagrange-Formulierung: Man beobachtet die Bewegung von jedem einzelnen Partikel bzw. einem Paket von Partikeln; es wird also die Bewegung eines Teils der Strömung verfolgt, wie er eine komplexe Trajektorie in einem turbulenten Fließmedium bildet (Längsschnittanalyse).

- Die Euler-Formulierung: Man beobachtet die Fließeigenschaften in

47) Anwendungen der atmosphärischen Dispersionsgleichung finden sich in Arbeiten von z. B. Calder (1949), Lebedeff, Hameed (1975), Sharma, Myrup (1975).

48) An dieser Stelle soll schon ein Hinweis darauf gegeben werden, wie in den folgenden Kapiteln die Diffusion modelliert wird:

$$
d S_{i} / d t=-\gamma_{i} E_{i}+\gamma_{j} E_{j}-\alpha_{i} S_{i}+E_{i}
$$

wobei $S_{i}$ die Konzentration der Schadstoffe in einer Region $i$ angibt, $E_{i}$ die in Region $i$ produzierten Emissionen, $\gamma_{i}$ den Anteil an Emissionen, der in die andere Region durch Diffusionsvorgänge gelangt und $\alpha_{i}$ die Assimilationsfähigkeit des Ökosystems, d. h. den Abbau der Substanz. Es wird nur ein eindimensionales Punktregionenmodell betrachtet. Es gilt dann in obiger Formulierung:

$c=S_{i}, s=E_{i}-\alpha_{i} S_{i}$, während in den Termen $\gamma_{i} E_{i}$ und $\gamma_{j} E_{j}$ die Ausbreitung der Schadstoffe durch Diffusion und Advektion subsumiert wird. Dadurch wird durch die Diffusionsfunktion in den Modellen das Gesetz der Erhaltung der Masse berücksichtigt, wie es auch bei diesen einfachen Dispersionsmodellen modelliert wird. 
einem bestimmten Punkt im Raum, ohne die Historie der Eigenschaften in Betracht zu ziehen, die mit der Unzahl der Fließpartikel einhergehen, die den fraglichen Punkt durchlaufen (Querschnittanalyse).

Diese zwei Methoden sind Konzepte, die man aus der physikalischen Strömungslehre abgeleitet hat. Die Beobachtung der Lagrange-Strömung von fließenden Partikeln ist jedoch entschieden schwieriger - wenn nicht gar unmöglich - als die Beobachtung der Euler-Strömung [vgl. Okubo (1980, 3)].

\subsubsection{Die Lagrange-Formulierung ${ }^{491}$}

Die Lagrange-Formulierung der Bewegung in einer Strömung hat Vorteile bei der Formulierung der turbulenten Dispersion, weil bei dieser Methode das Verhalten einzelner Fließpartikel beobachtet wird. Sei $r_{0}$ die Position des Partikels im Zeitpunkt $t_{0}$. Die nachfolgende Bewegung des Partikels wird durch den zeitabhängigen Positionsvektor $r=r\left(r_{0}, t_{0} ; t\right)$ repräsentiert; er gibt die Position des Partikels im Zeitpunkt $t$ an, dessen Ausgangsposition $r_{0}$ im Zeitpunkt $t_{0}$ war. $x, y$ und $z$ sind die Komponenten des Positionsvektors des Partikels und werden Lagrange-Koordinaten genannt.

Wir betrachten ein Experiment, bei dem eine Reihe markierter Partikel in einem festen Raumpunkt in ein turbulentes, homogenes und stationäres Strömungsfeld emittiert werden. Die markierten Partikel sind klein und werden neutral mitgetragen, so daß sie den Bewegungen des turbulenten Strömungsfeldes im Detail nachfolgen. Jedes Partikel verfolgt eine einzige gekrümmte Trajektorie, die die Bewegung anderer Partikel nicht nachahmt, die zu einem früheren oder späteren Zeitpunkt emittiert werden. Obwohl der Hauptstrom in $x$-Richtung geht, erreichen die verschiedenen Partikel nicht den gleichen Punkt nach einem bestimmten Zeitraum, da sie unterschiedliche turbulente Trajektorien haben. Die Endposition der Partikel ist eine gestreute Menge, die quantifiziert werden soll. Dazu wird angenommen, daß die Geschwindigkeit jedes Partikels eine bekannte

49) Vgl. zur Entwicklung des Lagrange-Ansatzes z. B. Csanady (1973), Pasquill (1974), Seinfeld (1975). 
Funktion der Zeit ist. Die $y$-Geschwindigkeitskomponente des $i$-ten Partikels ist $u_{y i}(t)$ und die $y$-Position des Partikels nach einem bestimmten Zeitraum $\theta_{0}$, wenn es in $t_{i 0}$ emittiert wurde, ist gegeben durch

$$
y_{i}\left(t_{i}\right)=\int_{0}^{\theta_{0}} u_{y i}\left(t_{i 0}+\theta\right) d \theta
$$

Dann wird die durchschnittliche $y$-Koordinate für $N$ Partikel, die in den Zeitpunkten $t_{10}, t_{20}, \ldots, t_{N 0}$ emittiert wurden, berechnet:

$$
\mathrm{y}=\frac{1}{N} \sum_{i=1}^{N} y_{i}\left(t_{i}\right)=\frac{1}{N} \sum_{i=1}^{N} \int_{0}^{\theta_{0}} u_{y i}\left(t_{i 0}+\theta\right) d \theta
$$

Dies ist äquivalent zu

$$
\mathbf{y}=\int_{0}^{\theta}\left|\frac{1}{N} \sum_{i=1}^{N} u_{y i}\left(t_{\iota 0}+\theta\right)\right| d \theta \equiv \int_{0}^{\theta}\left\langle u_{y}\left(t_{i 0}+\theta\right)\right\rangle d \theta
$$

wobei $\left\langle u_{y}\left(t_{i 0}+\theta\right)\right\rangle$ die durchschnittliche Lagrange $y$-Komponente der Geschwindigkeit darstellt, die durch die Beobachtung einer großen Anzahl von Partikeln im Zeitpunkt $\theta$ nach ihrer Emission geformt wird. Dieser Durchschnitt soll Null sein (Ergodische Hypothese), d. h. im Durchschnitt sollen sich die Partikel nicht in $y$-Richtung ausbreiten (vgl. Schaubild 2.3). Jedoch existiert eine Streuung um diesen Mittelwert, ausgedrückt durch die Varianz $\sigma^{2}{ }_{y}$ :

$$
\mathrm{o}_{y}^{2}=\frac{1}{N} \sum_{i=1}^{N} y_{i}^{2}\left(t_{i 0}+\theta\right)
$$

Obwohl die Turbulenz stationär ist, steigt $\sigma^{2}{ }_{y}$ mit der Zeit und wird deshalb evolutionäre Größe genannt. Aus (2.9) erhalten wir nach einigen Umformungen und einer Änderung der Zeitskala $\left(\xi=\theta_{0}-\theta\right)$ :

$$
\frac{d \sigma_{y}^{2}}{d \theta_{0}}=2 \int_{0}^{\theta_{0}}\left\langle u_{y i}\left(t_{i}\right) u_{y i}\left(t_{i}-\xi\right) d \xi\right.
$$

Mit Hilfe von (2.16) wird die Lagrange-Autokorrelationsfunktion $A_{y}(\xi)$ abgeleitet: 
(2.17)

$$
A_{y}(\xi)=\frac{\left\langle u_{y}(t) u_{y}(t+\xi)\right\rangle}{\left\langle u_{y}^{2}(t)\right\rangle}
$$

Dabei gilt: Für $\xi \rightarrow 0$ folgt $A_{y}(\xi) \rightarrow 1$ und für große $\xi$ geht $A_{y}(\xi)$ gegen Null; d. h., die $y$-Geschwindigkeit hängt für große $\xi$ nicht stark von weiter zurückliegenden Bewegungen ab. Bei stationärer Turbulenz ist $A_{y}(\xi)$ konstant für alle Zeitpunkte $t$, damit ist $A_{y}(\xi)$ eine zur Geraden $\xi=0$ symmetrische Funktion: $A_{y}(\xi)=A_{y}(-\xi)$. Die Form $\operatorname{der} A_{y}(\xi)$-Funktion ist in Schaubild 2.4 dargestellt.

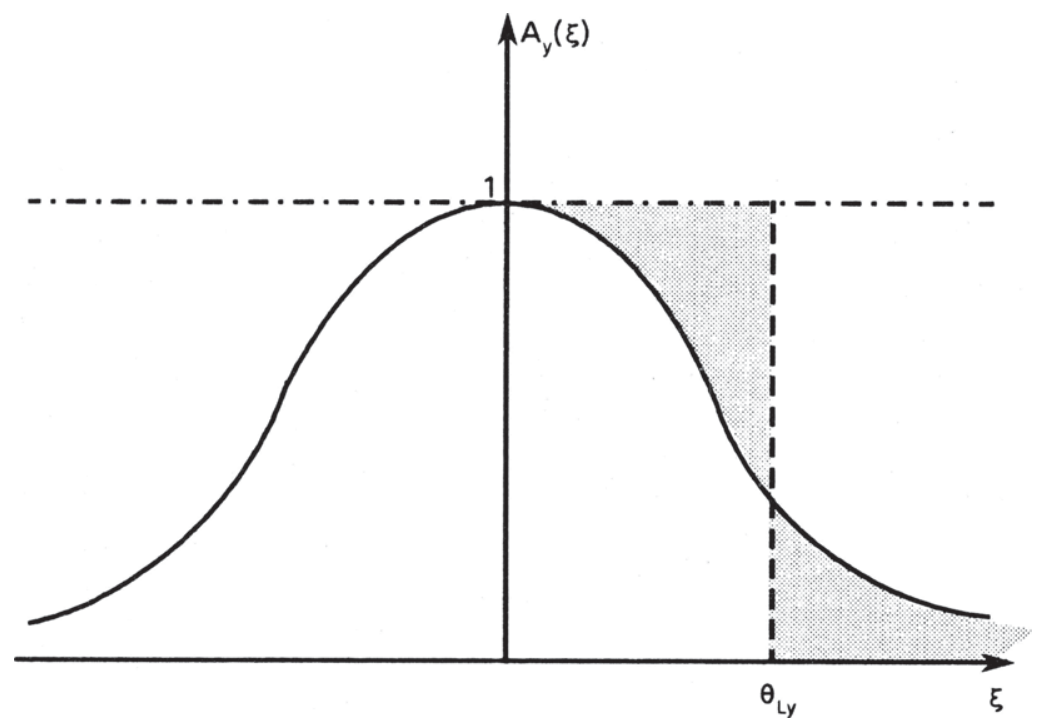

Schaubild 2.4: $\operatorname{Die} A_{y}(\xi)$-Funktion

Die Lagrange-Autokorrelation ist, da Beobachtungen, wenn überhaupt, nur unter großen Schwierigkeiten gewonnen werden können, nur schwer zu bestimmen.

Eine wichtige Eigenschaft der Funktion läßt sich über die Lagrange-Zeitskala $\theta_{L y}$ bestimmen: 
Für $\xi=\theta_{L y}$ sind die schraffierten Flächen über bzw. unter $\operatorname{der} A_{y}(\xi)$-Kurve in Schaubild 2.4 gleich, es gilt

$$
\int_{0}^{\theta_{L y}}\left[1-A_{y}(\xi)\right] d \xi=\int_{\theta_{L y}}^{\infty} A_{y}(\xi) d \xi
$$

oder

$$
\theta_{L y}=\int_{0}^{\infty} A_{y}(\xi) d \xi
$$

Das statistische Verhalten der Partikel kann nun mit Hilfe der LagrangeZeitskala folgendermaßen beschrieben werden: Für $\xi \ll \theta_{L y}$ ist die Partikelbewegung selbstkorreliert und für $\xi \gg \theta_{L y}$ versch windet die Autokorrelation. Es gibt also zwei unterschiedliche Ausprägungen der turbulenten Dispersion, die sich stark unterscheiden. Je weiter der Emissionszeitpunkt zurückliegt, desto unwichtiger wird, wann und wo emittiert wurde und die Dispersion hängt von der turbulenten Bewegung allein ab. Liegt der Emissionszeitpunkt noch nicht weit zurück, dann ist die Partikelbewegung auch von den seitherigen Bewegungen mit abhängig.

\section{DasGauß-Rauchfahnen-Modell}

Folgende Voraussetzungen müssen erfüllt sein, damit das Gauß-Rauchfahnen-Modell angewandt werden kann [vgl. Sutton (1953)]:

(i) Quasikonstanz der Winde. Diese Annahme ist nur aufrecht zuerhalten, wenn kurze Zeitperioden und begrenzte geographische Ausdehnungen betrachtet werden.

(ii) Keine chemischen Reaktionen der Schadstoffe. Die meisten chemischen Reaktionen verlaufen relativ langsam, so daß auch diese Annahme rechtfertigbar erscheint.

(iii) Vertikale Konstanz der Windgeschwindigkeiten $u_{x}, u_{y}, u_{z}$. Nicht nur in horizontaler Richtung (Annahme (i)) muß die Windgeschwindigkeit konstant sein, sondern es darf überhaupt nur eine Windgeschwindigkeit in der gesamten betrachteten Grenzschicht der Atmosphäre geben.

Das Gauß-Rauchfahnen-Modell basiert auf der Approximation, daß die Konzentration eines Schadstoffes in Windrichtung, der von einer Punkt- 
quelle emittiert wurde, in der atmosphärischen Grenzschicht Gaußverteilt ist, jedoch mit unterschiedlichen Streuungen (Dispersionskoeffizienten) in horizontaler und vertikaler Richtung. Dies ist in Schaubild 2.5 dargestellt.

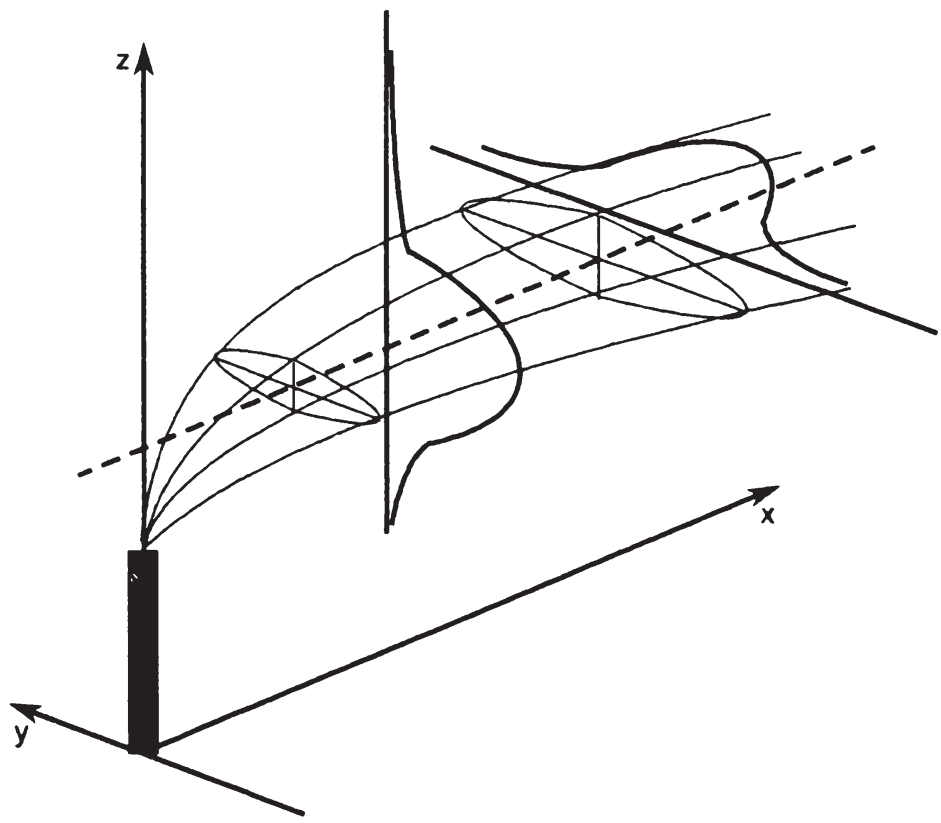

Schaubild 2.5: Das Gauß-Rauchfahnen-Modell

Es gilt also

$$
F(x, y, z)=\exp \left[-\frac{y^{2}}{2 \sigma_{y}^{2}}-\frac{z^{2}}{2 \sigma_{z}^{2}}\right]
$$

wobei $\sigma_{y}$ und $\sigma_{z}$ die von $x$ abhängigen Dispersionskoeffizienten in horizontaler bzw. vertikaler Richtung sind bzw. die mittleren quadratischen Abweichungen, wie wir sie in Gleichung (2.9) definiert haben. Jedoch ist die Annahme der isotropen, homogenen Turbulenz aufgegeben, so daß die Dis- 
persionskoeffizienten über Feldbeobachtungen bestimmt werden müssen und - zumindest im Prinzip - nicht berechnet werden können.

$F(x, y, z)$ hat entlang der Fahnen-Achse $(y=z=0)$ den Wert Eins. $F$ repräsentiert also die Größe, die mit der axialen Konzentration $c(x, 0,0)$ multipliziert werden muß, um die mittlere Konzentration in jedem Punkt zu erhalten:

$$
c(x, y, z)=c(x, 0,0) F(x, y, z)
$$

$c(x, 0,0)$ erhält man durch die Anwendung des Gesetzes der Erhaltung der Masse: Die Schadstoffquellenstärke $Q_{p}$ ist gleich der Strömungsrate durch jede Fläche senkrecht zur $x$-Achse. Formal heißt dies

$$
Q_{p}=u_{x} c(x, 0,0) \int_{-\infty}^{\infty} \int_{-\infty}^{\infty} F(x, y, z) d y d z
$$

Integration ergibt

$$
c(x, 0,0)=\frac{Q_{p}}{2 \pi u_{x}^{0} y_{z}}
$$

Die grundlegende Gauß'sche Rauchfahnen-Formel erhält man durch Einsetzen von Gleichung (2.22) in Gleichung (2.20)

$$
c(x, y, z)=\frac{Q_{p}}{2 \pi u_{x} \sigma_{y} \sigma_{z}} \exp \left|-\frac{y^{2}}{2 \sigma_{y}^{2}}-\frac{z^{2}}{2 \sigma_{z}^{2}}\right|
$$

Gleichung (2.23) gibt die Konzentration an, von einer Punktquelle aus dem Ursprung $(0,0,0)$ ausgehend, in einem Medium von unendlicher Ausdehnung, falls die Dispersionskoeffizienten geeignet gewählt wurden.

Befindet sich die Punktquelle nicht im Ursprung (der Erdoberfläche), sondern in der Höhe $H$ über der Erdoberfläche, wird das Koordinatensystem so transformiert, daß $z$ durch $(z-H)$ ersetzt wird. Jedoch muß noch ein weiterer Term hinzugenommen werden, da für $z=0$ angenommen wird, daß es keine Dispersion in den Boden hinein gibt $(\partial c / \partial z=0)$ und dies für $(z-H)$ nicht mehr gilt. Eine spiegelbildliche Quelle in $(0,0,-H)$ wird angenommen, die die Diffusion nach unten ausdrücken soll. Dann gilt 


$$
c(x, y, z)=\frac{Q_{p}}{2 \pi u_{x} \sigma_{y} \sigma_{z}}\left\{\exp \left[-\frac{y^{2}}{2 \sigma_{y}^{2}}-\frac{(z-H)^{2}}{2 \sigma_{z}^{2}}\right]+\exp \left[-\frac{y^{2}}{2 \sigma_{y}^{2}}-\frac{(z+H)^{2}}{2 \sigma_{z}^{2}}\right]\right\}
$$

Setzt man in (2.24) $z=0$, so erhält man die Oberflächenkonzentrationsverteilung auf der unteren Begrenzungsfläche

$$
c(x, y, 0)=\frac{Q_{p}}{2 \pi u_{x} \sigma_{y} \sigma_{z}} \exp \left[-\frac{y^{2}}{2 \sigma_{y}^{2}}-\frac{H^{2}}{2 \sigma_{z}^{2}}\right]
$$

Das Gauß-Rauchfahnen-Modell, das oben in seiner einfachsten Form dargestellt wurde, ist für viele Multiquellen-Dispersionsmodelle erweitert worden. Aufgrund ihrer Einfachheit werden Gauß-Rauchfahnen-Modelle oft angewandt [vgl. Runca u. a. (1979, 3); z. B. Pooler (1961), Calder (1971), Martin (1971), Runca u. a. (1976)].

Eine Anwendung des Gauß-Modells ist im Referentenentwurf vom Juni 1982 zur Änderung der TA-Luft vorgesehen. Es soll bei der Genehmigung von Anlagen überall in der BR Deutschland verwendet werden [vgl. BMI (1982 (92), 24)]. Dabei sollen insbesondere die Zusatzbelastungen durch die Emissionen neuer Anlagen errechnet werden können unter Berücksichtigung der Wetter- und Klimaverhältnisse. Das Verfahren hat jedoch auch seine Grenzen, wie in BMI (1982 (92), 24) angemerkt wird, da es für die Modellierung des weiträumigen Transports nicht geeignet erscheint.

Allgemein läßt sich zu der Lagrange-Formulierung sagen, daß die Annahmen so einschränkend sind, daß eine "gute" Abbildung der Wirklichkeit nicht zu erreichen ist. Vor allem durch die Annahmen, daß die Windgeschwindigkeit konstant ist und keine chemischen Reaktionen zwischen den Schadstoffen und den anderen Bestandteilen der Atmosphäre vorkommt, hat dazu geführt, neue Modelle zu entwickeln, die den komplexen Sachverhalten besser Rechnung tragen [vgl. Van Dop $(1983,52)$ ]. 


\subsubsection{Die Euler-Formulierung}

Hier beschränken wir uns auf die Darstellung der Grundzüge der sogenannten Zellen- oder Schachtelmodelle. Die Verschmutzungskonzentration in einer Mischschicht der Atmosphäre ist manchmal relativ uniform in senkrechter Richtung und der Transport wird primär durch Advektion hervorgerufen. Diese Situation kann erwartet werden, falls die konvektive Aktivität gemäßigt zwischen einer wohldefinierten niedrigliegenden Inversionsschicht stattfindet. Unter diesen Umständen kann die Verschmutzungskonzentration analysiert werden, indem die Luftschicht in eine Folge verbundener Zellen oder Schachteln aufgeteilt wird. Turbulentes Mischen wird als effizient betrachtet, so daß jede Zelle einen "wohl-bewegten" Reaktor repräsentiert, für die man die chemische Produktion oder den chemischen Abbau von Verschmutzung errechnen kann.

Wird die Luftschicht in viele Zellen eingeteilt, kann in das Modell eine große Anzahl Details regionaler meteorologischer Eigenschaften, chemischer Reaktionsfolgen und anderes aufgenommen werden.

Ein flexibles Vielzellenmodell von beträchtlicher Allgemeinheit wurde von Kyan und Seinfeld (1973) formuliert:

Die Masseerhaltung der $i$-ten Substanz in einer Mischung von Schadstoffen in der $k$-ten Zelle ist gegeben durch

$$
\frac{d}{d t}\left(C_{i k} V_{k}\right)=+\sum_{j} L_{j k} C_{i j}-C_{i k} \sum_{l} L_{k l}+E_{i k}-d_{i}+r_{i}
$$

Der Term auf der linken Seite drückt die Änderung der Masse der $i$-ten Substanz in der $k$-ten Zelle aus, deren momentanes Volumen $V_{k}{ }^{50)}$ ist. Das Subskript $j$ steht für die Zellen, von denen die Zelle $k$ etwas erhält und das Subskript $l$ steht für die Zellen, denen die Zelle $k$ etwas abgibt. Die Volumenströme in und aus der $k$-ten Zelle werden mit $L_{j k}$ bzw. $L_{k l}$ bezeichnet. Die aggregierte Quellenstärke für alle Quellen der $i$-ten Komponenten der $k$-ten Zelle ist $E_{i k}$, die chemische Produktionsrate ist durch $r_{i}$ gegeben und

50) $V_{k}$ kann sich ebenfalls åndern, z. B. wenn die Zelle bis zum Gipfel der Mischschicht reicht, deren Höhe mit der Zeit variiert. 
$d_{i}$ ist die Abbaurate. Die Zellen sind i. a. von konstanter rechteckiger horizontaler Fläche $a_{k}$, deshalb gilt

$$
\frac{d V_{k}}{d t}=a_{k} \frac{d H_{k}}{d t}
$$

wobei $H_{k}$ die Höhe der Zelle ist.

Graedel et al (1976) haben solch ein Vielzellenmodell auf das nördliche New Jersey angewandt und analysieren damit eine komplexe photochemische Reaktionsfolge, die 76 Substanzen enthält, die durch 143 chemische Reaktionen verbunden sind.

Tennekes (1976) hat aus Gleichung (2.25) ein Einzellenmodell für chemisch inaktive Stoffe abgeleitet. Die Zelle hat die Dimension $D \times D \times H_{c}{ }^{51)}$, in der eine Schadstoffquelle der Stärke $Q_{a}$ liegt. Die Windgeschwindigkeit $u$ wird in diesem Fall als konstant in der Zeit betrachtet und senkrecht zu zwei gegenüberliegenden Seiten der Zelle.

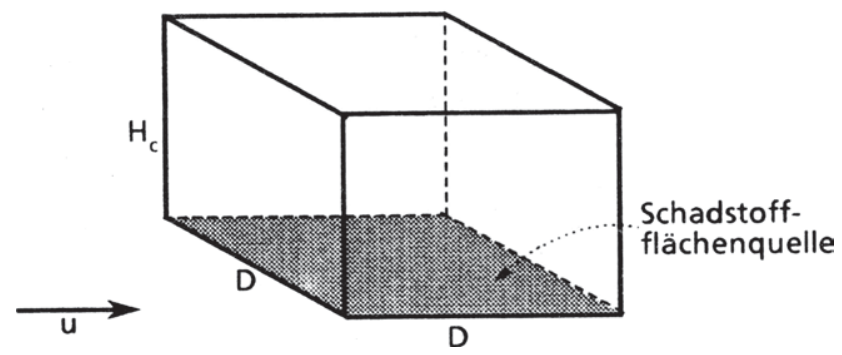

Schaubild 2.4: Das Einzellenmodell

Die Konzentration $C$ wird als einheitlich angenommen, während die Kon51) z. B. eine Stadtregion. 
zentration über der Zelle $C^{*}$ ist. Die Höhe der Zelle $H_{c}$ ist eine zeitabhängige Größe, die entweder die Höhe ist, bis zu der die Schadstoffe verteilt werden durch die turbulente Dispersion $H_{p}$ oder, falls die Windgeschwindigkeit sehr klein ist, die Höhe der Mischschicht $H_{m}$. Turbulente Dispersion von den Seiten der Schachtel wird ignoriert. Der Schadstofffluß aus der Box heraus in Windrichtung ist $C u H_{c} D$ und der Schadstofffluß in die Box hinein muß nicht betrachtet werden, da dieser nur eine zusätzliche additive Konstante wäre. Man erhält dann

$$
\frac{d}{d t}\left(C D^{2} H_{c}\right)=C^{*} D^{2} \frac{d H_{c}}{d t}-C u H_{c} D+Q_{a} D^{2}
$$

d. h. die Masseänderungsrate des Schadstoffs in der Zelle steigt durch die Höhensteigerung der Mischschicht, sinkt jedoch durch den Abfluß an der Windrichtungsseite und steigt durch die Emissionen der Schadstoffquelle. Dividiert man (2.28) durch $D^{2}$, erhält man

$$
H_{c} \frac{d C}{d t}=Q_{a}-\left(C-C^{*}\right) \frac{d H_{c}}{d t}-\frac{u H_{c} C}{D}
$$

Eine wichtige Eigenschaft dieses Schachtel-Modells ist, daß es mit der Dynamik der konvektiven, "angetriebenen" Mischschicht in Verbindung gebracht werden kann und deshalb die Möglichkeit besitzt, die Konzentrationsabfolge zu berechnen, um damit die Dynamik der Konvektion in der atmosphärischen Grenzschicht geeignet zu betrachten.

Hamlen (1978) hat ein solches Zellenmodell in ein ökonomisches Modell integriert. Er betrachtet die Diffusion von Schadstoffen, die von einer Stadt emittiert werden und durch Luftzellen mit abnehmender Konzentration in Hauptwindrichtung verteilt werden. Er analysiert statt eines intertemporalen ein räumliches Kontrollmodell, wobei die Hauptwindrichtung die Rolle der Zeit übernimmt. Damit zeigt er, daß es optimal ist, eine Emissionsteuer räumlich zu differenzieren.

\subsubsection{Abschließende Bemerkung}

Zum Abschluß sei noch darauf hingewiesen, daß hier nur die grundlegenden Formulierungen des Lagrange- und des Euler-Ansatzes der Diffusi- 
ons-Advektions-Modellierung dargestellt wird. Es gibt Ansätze, die den Euler- und den Lagrange-Ansatz verbinden, z. B. die sogenannten "particle-in-cell" Methode [vgl. z. B. Sklarew et al (1971), Lange (1973)], die Galerkin-Methode [vgl. z. B. Melli (1976), Christensen, Prahm (1976)], sowie den "fractional step" Algorithmus [vgl. z. B. Runca et al (1979)]. Diese Modelle geben eine adäquate Beschreibung des Diffusionsphänomens, sind jedoch mit großen "Input"-Unsicherheiten belastet. Darüberhinaus haben zwar die Parameter (Diffusionskoeffizienten) eine eindeutige physikalische Bedeutung, jedoch beruhen sie auf manchmal fragwürdigen meteorologischen Annahmen. Schließlich erfordern Advektions-Diffusionsmodelle komplexe numerische Lösungskonzepte. "The overall result ist usually an unsatisfactory episode forecast performance requiring a relatively large amount of effort" [Fronza et al $(1979,24)]$.

Es erscheint dann sinnvoll zu sein, die in den folgenden Kapiteln benutzte Diffusions-Advektions-Gleichung möglichst einfach zu halten, da das Ziel dieser Arbeit ist, grundsätzliche ökonomische Zusammenhänge bei Vorliegen von Schadstoffdiffusion aufzuzeigen. Die angesprochenen Schwierigkeiten bei der Analyse von Diffusionsvorgängen würden sich auf die Analyse übertragen und somit einen nicht gerechtfertigten Mehraufwand an Komplexität erfordern. 


\section{Kapitel 3: Das Grundmodell}

Bei den Modellrechnungen betrachten wir eine Ökonomie, die aus zwei Regionen besteht. Der Modellrahmen wird von den üblichen Ein-RegionUmwelt-Modellen übernommen ${ }^{1}$. Mit leichten Modifikationen werden diese Modelle dahingehend erweitert, daß sie auf eine Zwei-RegionenÖkonomie angewandt werden können. Im folgenden wird die allgemeine Ökonomie beschrieben, die im Grundmuster für alle folgenden Modellvarianten gilt (Abschnitt 1). In Abschnitt 2 wird dieses Modell zur Beantwortung der Frage herangezogen, ob es optimal sein kann, identische Emissionssteuern zu erheben, wenn unterschiedliche regionale Gegebenheiten vorliegen.

\section{DIE MODELLÖKONOMIE}

Die Grundstruktur der Beziehungen innerhalb einer Region sind in Schaubild 3.1 dargestellt.

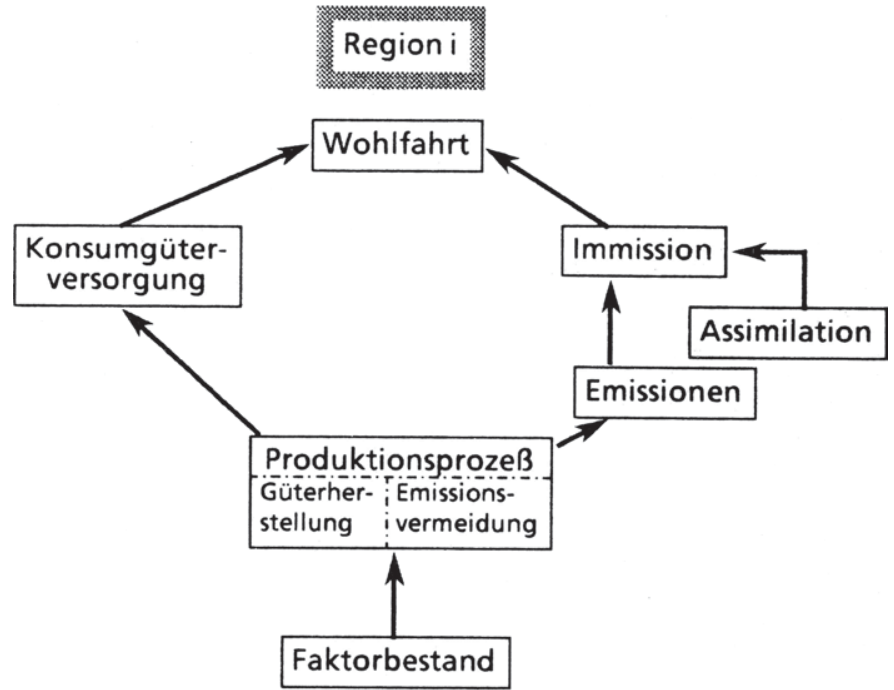

Schaubild 3.1: Die Grundstruktur der Beziehungen innerhalb einer Region

1) Vgl. z. B. d'Arge, Kogiku (1973), Vogt (1981). 


\subsection{Die Produktionstechnologie}

Die Produktionstechnologie der Region $i(i=1,2)$ wird folgendermaßen spezifiziert ${ }^{2}$ : Bei der Herstellung des Gutes $X_{i}$ durch den Einsatz des Faktors $R_{i}$ fällt eine umweltschädigende Kuppelproduktmenge $E_{i}$ an, die weder für den Konsum noch für die Produktion verwertbar ist. Diese Produktionstechnologie wird durch die streng konkave, zweifach stetig differenzierbare Produktionsfunktion $f$ dargestellt:

$\left.X_{i}(t) \leq f \mid R_{i}(t), E_{i}(t)\right\}$

mit dem konvexen Definitionsbereich

$$
D\left(\gamma^{i}\right)=\left\{\left(R_{i}(t), E_{i}(t)\right) \in \mathbf{R}_{+}^{2} \wedge R_{i}(t) \geq \frac{1}{a_{i}} E_{i}(t), a_{i}>0 \text { und konstant }\right\}
$$

und den Eigenschaften

$$
\begin{aligned}
& f\left[0, E_{i}(t)\right]=0, f\left[R_{i}(t), 0\right] \geq 0 \forall R_{i}(t)>0 ; f_{R}^{i}\left[R_{i}(t), E_{i}(t)\right]>0, \\
& f_{E^{\prime}}^{i}\left[R_{i}(t), E_{i}(t)\right]>0^{3)} .
\end{aligned}
$$

Weiter soll gelten, daß $f$ bezüglich dem Faktor $R_{i}$ homogen vom Grade $k<1$ ist.

Dieser sogenannte "Nettoansatz"4) betrachtet die Emissionen $E_{i}(t)$ formal als zusätzlichen Produktionsfaktor, der die Inanspruchnahme der Umwelt als Schadstoffaufnahmemedium durch den Produktionsprozeß ausdrückt. Es werden nur diejenigen Emissionen betrachtet, die tatsächlich an die Umwelt abgegeben werden, d. h. sektorinterne Entsorgungsaktivitäten werden durch diese Produktionstechnologie implizit berücksichtigt.

2) Vgl. Klevorick, Kramer $(1973,106 \mathrm{ff}$. Eine allgemeinere Form dieser Produktionstechnologie wird in Pethig (1975, 100-106) entwickelt. Vgl. auch Pethig (1979, 16-26).

3) $f_{R} \equiv \partial f / \partial R_{i}$ und $f_{E} \equiv \partial f / \partial E_{i}$. Diese Vorgehensweise gilt auch analog für andere Funktionen.

4) "Net emissions approach", vgl. Siebert, Eichberger et al $(1980,29-42)$. Ein alternativer Ansatz ist das sogenannte Bruttokonzept ("gross emissions approach"), bei dem explizit eine Schadensfunktion berücksichtigt wird. Den Zusammenhang zwischen Brutto- und Nettokonzept zeigen Siebert, Eichberger et al (1980, 42-52). 
Die Bedingung

$$
R_{i}(t) \geq \frac{1}{a_{i}} E_{i}(t)
$$

ordnet alternativen Faktoreinsätzen die höchstmögliche Kuppelproduktmenge zu, sie drückt also die Kuppelproduktionskapazität aus. In Schaubild 3.2 wird der Produktionsraum der Region $i$ dargestellt.

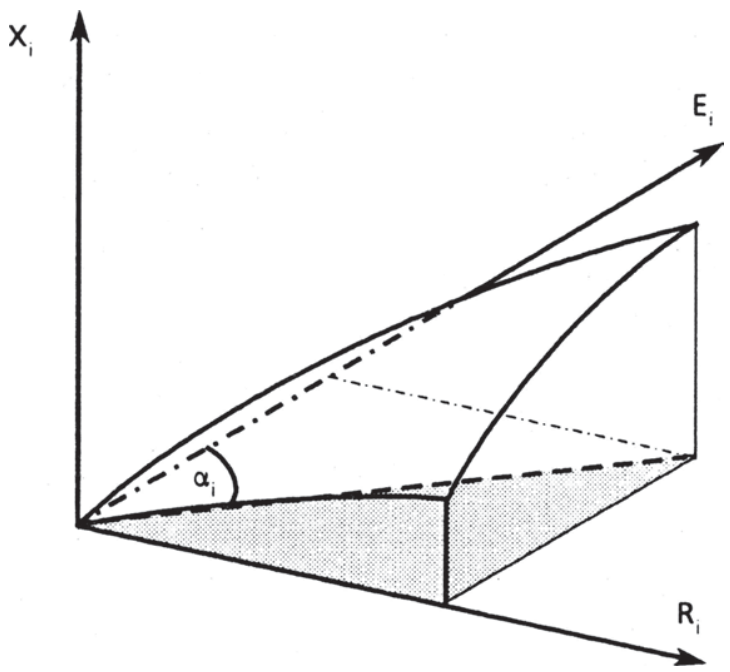

Schaubild 3.2: Der Produktionsraum der Region $i$

In der Region $i$ stehen in jedem Zeitpunkt $t \mathbf{R}_{i}$ Ressourcen zur Verfügung. Sind diese Faktoren immobil ${ }^{5}$, gilt die Faktorrestriktion

$$
\mathbf{R}_{i}-R_{i}(t) \geq 0 .
$$

Sind die Faktoren mobil ${ }^{6}$, so ist bei annahmegemäß konstantem Weltmarktpreis keine Faktorrestriktion vorhanden, sondern die Begrenzung erfolgt über die Zahlungsbilanzrestriktion.

5) In Kapitel 3 und 4.

6) In Kapitel 5 und Kapitel 6. 


\subsection{DAS ÖKOSYSTEM}

Die zeitliche Immissionsänderungsrate $d S_{i} / d t$ in Region $i$ ist abhängig von den Emissionen und der Assimilationsfähigkeit in Region $i$ und dem Teil der Emissionen von Region $j$, der nach Region $i$ "exportiert" wird:

$$
\frac{d S_{i}(t)}{d t}=\left(1-Y_{i}\right) E_{i}(t)+Y_{j} E_{j}(t)-a_{i} S_{i}(t)
$$

mit $\alpha_{i} \in[0,1]$ und konstant, dem Assimilationskoeffizienten der Region $i^{7)}$, und $\gamma_{i} \in[0,1)$ und konstant, dem Anteil der Emissionen der Region $i$, der durch Diffusionsvorgänge das Ökosystem der Region $j$ beeinflußt. Aus den Gleichungen (3.4) lassen sich stationäre Ökozustand-Funktionen herleiten, mit deren Hilfe die Menge der Emission-Immission-Kombinationen hinsichtlich ihrer ökologischen Eigenschaften unterteilt werden kann. Unter einem stationären Zustand des Ökosystems versteht man die Gleichheit von assimilationsbedingter Immissionsabnahme und emissionsbedingter Immissionszunahme ${ }^{8}$.

Aus (3.4) erhält man

$$
S_{i}(t)=\frac{1}{a_{i}}\left[\left(1-Y_{i}\right) E_{i}(t)+Y_{j} E_{j}(t)\right]
$$

In Schaubild 3.3 ist die stationäre Ökozustand - Funktion für Region $i$ bei alternativen $E_{j}(t)$-Werten dargestellt. Aus Schaubild 3.3 sieht man, daß der stationäre Zustand des Ökosystems der Region $i$ von den Emissionen der Region $j$ mitabhängt. Je größer die Emissionen der Region $j$ sind, desto weiter nach rechts versehiebt sich die stationäre Ökozustand - Funktion der Region $i$, d. h. je größer $E_{j}(t)$ ist, desto kleiner muß $E_{i}(t)$ sein, um einen bestimmten stationären Ökozustand $S_{i} z u$ halten. Die Steigung dieser Funktion ist sowohl von der Assimilationsfähigkeit des Ökosystems der

7) Die Annahme, daß der Assimilationskoeffizient konstant ist, wird zur Vereinfachung der Analyse eingeführt. Eine allgemeinere Form der Assimilationsfähigkeit des Ökosystems wäre etwa $Q(S) \operatorname{mit} Q_{S}>0$ :

8) Vogt $(1981,14)$ spricht in diesem Zusammenhang von ökologischem Gleichgewicht, vgl. auch SRU $(1978,20)$. 


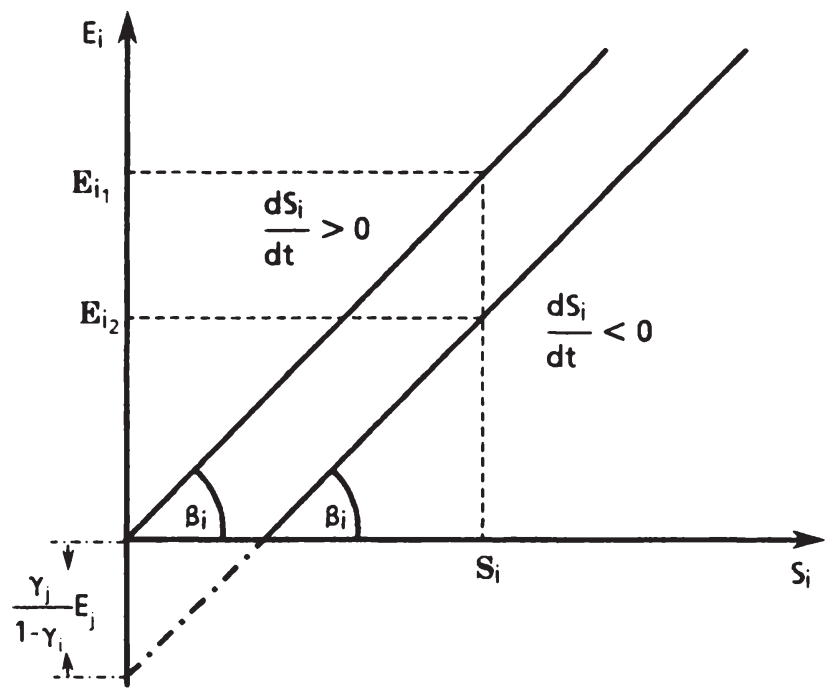

Schaubild 3.3: Stationäre Ökozustand-Funktion der Region $i$

Region als auch von dem Diffusionskoeffizienten abhängig. Je höher der Diffusionskoeffizient $\gamma_{i}$ ist, d. h. je mehr Emissionen $E_{i}(t)$ nach Region $j$ gelangen, desto steiler ist die stationäre Ökozustand-Funktion. Oberhalb dieser Funktion nehmen die Immissionen zu $\left[d S_{i} / d t>0\right]$, während sie unterhalb abnehmen $\left[d S_{i} / d t<0\right]$.

\subsection{DIE HANDELSBEZIEHUNGEN}

Die regionale Überschußnachfrage nach der Ressource $R_{i}(t)$ ist

$$
M_{i}(t)=R_{i}(t)-\mathbf{R}_{i}
$$

und die Überschußnachfrage nach dem Konsumgut ist

$$
N_{i}(t)=C_{i}(t)-X_{i}(t)
$$

wobei $C_{i}(t)$ der Konsum des Gutes $X$ in Region $i$ bedeutet. 
Sei $q_{R}(t)$ der Preis der Ressource auf dem Faktormarkt und $q_{X}(t)$ der Preis des Konsumgutes auf dem Gütermarkt. Wir gehen davon aus, daß zwischen den Regionen keine Kreditbeziehungen bestehen, so daß die "Zahlungsbilanz" jeder Region zu jedem Zeitpunkt ausgeglichen sein muß:

$$
q_{R}(t) M_{i}(t)+q_{X}(t) N_{i}(t)=0
$$

Sei

$$
q(t)=\frac{q_{R}(t)}{q_{X}(t)}=\mathbf{q} \quad q_{X}(t)>0 \forall t \in[0, \infty)
$$

der Relativpreis, der vom Weltmarkt vorgegeben ist und damit für beide Regionen gilt. Die Zahlungsbilanzbedingung kann dann umgeformt werden zu

$$
C_{i}(t)-X_{i}(t)+\mathbf{q}\left[R_{i}(t)-\mathbf{R}_{i}\right]=0 .
$$

Die so definierten Handelsbeziehungen der Regionen sind in dem Sinne sehr einfach, daß nur ein Gut und ein Produktionsfaktor gehandelt werden. Man kann zeigen, daß, wenn mit Hilfe des Faktors ein zweites Konsumgut produziert wird, bei dem im Herstellungsprozeß keine Emissionen anfallen, sich äquivalente Implikationen ergeben, jedoch mit einer weiteren Komplexität der Analyse, so daß dieser Vorgehensweise hier nicht gefolgt wird.

\subsection{DIE WOHLFAHRTSFUNKTIONEN}

Die im folgenden vorgenommenen modelltheoretischen Analysen gehen von dem Konzept einer sozialen Wohlfahrtsfunktion aus. Dieses Konzept ist in der Literatur nicht unumstritten, da nach Arrow (1963) für plausible Annahmen für eine nicht-diktatorielle Gesellschaftsordnung eine soziale Wohlfahrtsfunktion nicht widerspruchsfrei abgeleitet werden kann. Warum hier trotzdem diesem Ansatz gefolgt wird, ergibt sich aus der Beobachtung, da $\beta$ "national decisions are made daily on the basis of something other than random selection" [Van Zele $(1978,19)]$. Uns interessiert hier 
auch nicht, wie eine solche Wohlfahrtsfunktion zustande kommt, sei es über einen institutionell verankerten Abstimmungsprozeß (wie z. B. in Dudenhöffer (1983) abgeleitet) oder seien es die Präferenzen eines Diktators, sondern es wird davon ausgegangen, daß es für regionale bzw. zentrale Behörden eine Entscheidungsgrundlage gibt, die es ermöglicht, rationale und in sich stimmige Entscheidungen zu treffen [vgl. hierzu auch Mäler (1974, 104-105), Vogt (1981, 20-25)].

Im folgenden wird also davon ausgegangen, daß die Wohlfahrt der Region $i$ im Zeitpunkt $t$ abhängig sei vom Konsum des Gutes $X$ und der Verschmutzung $S$ der Region $i$ im Zeitpunkt $t$ :

$$
U_{i}(t)=u^{i}\left[C_{i}(t) \cdot S_{i}(t)\right]
$$

Diese Funktion sei mehrfach stetig differenzierbar, konkav und separabel und besitze außerdem folgende Eigenschaften: $u^{i}[0,0]=0$,

(a) $u^{i}{ }_{C}\left[0, S_{i}(t)\right]=\infty, u_{C}^{i}>0 \forall C_{i}>0$,

(b) $u_{S}^{i}\left[C_{i}(t), 0\right]=0, u_{S}^{i}<0 \forall S_{i}>0$.

Durch die Wohlfahrtsfunktionen wird die Bewertung der Umweltbehörde bzw. -behörden ausgedrückt, die sie unterschiedlichen Kombinationen von Güterkonsum und Schadstoffbestand in Region $i$ beimißt bzw. beimessen. In Schaubild 3.4 ist ein so definiertes Nutzengebirge dargestellt.

Durch die Separabilität der Nutzenfunktion wird die Traktierbarkeit der Modelle vereinfacht. Darüberhinaus können über die Interdependenzen zwischen Konsum und Verschmutzung nur Vermutungen angestellt werden, deren Signifikanz sich nicht auf empirische Nachweise stützen kann, so daß diese üblicherweise vernachlässigt werden [vgl. z. B. d'Arge, Kogiku $(1974,64)$, Bender $(1976,250)$, Vogt $(1981,36)]^{9)}$. Durch Bedingung (3.9a) wird sichergestellt, daß eine optimale Politik immer ein positives Konsumniveau gewährleistet, während durch Bedingung (3.9b) vorausgesetzt wird, daß ein infinitesimal kleiner Verschmutzungsbestand keine

9) Vgl. aber hierzu Neher (1983), der die Abhängigkeit eines intertemporalen Gleichgewichts von den Kreuzableitungen der Nutzenfunktion untersucht. 


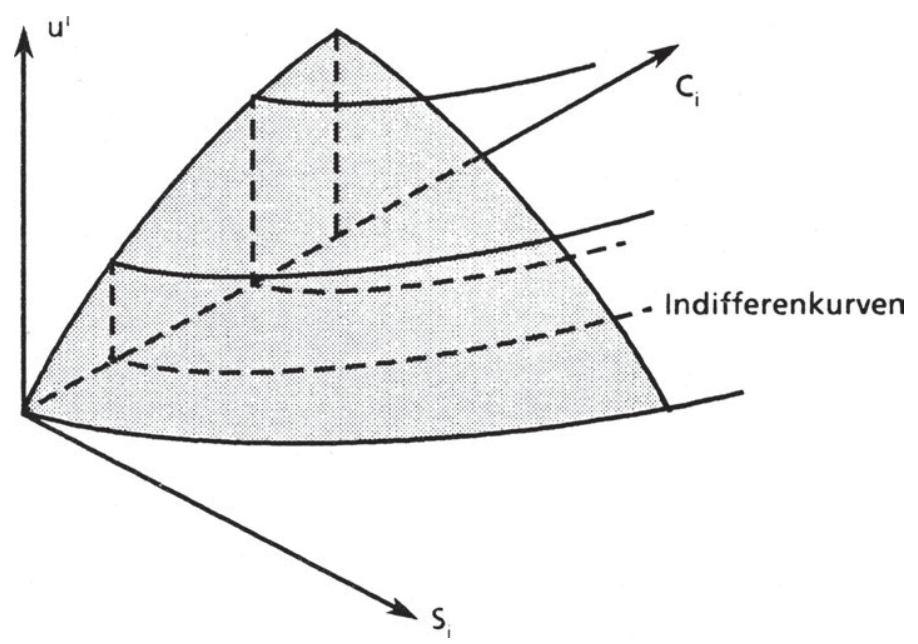

Schaubild 3.4: Die Nutzenfunktion

negativen Wohlfahrtseffekte bewirkt. Diese Bedingung ist hinreichend, daß auf dem Optimalpfad $S_{i}>0$ gilt [vgl. Forster (1973, 534-545)].

Der gesellschaftliche Planungshorizont wird in den nachfolgenden Analysen durchweg als unendlich angenommen. Bei der Festlegung dieses Zeithorizontes ist ein Werturteil gegeben, das sich als altruistisch apostrophieren läßt, da der Nutzen aller zukünftiger Generationen mit einbezogen wird [vgl. Inagaki $(1970,61)$, Vogt $(1981,23)]$. "Ein endlicher, auf die Lebenserwartung einer oder mehrerer Generationen begrenzter Zeithorizont wäre die realistischere Planungsalternative" [Bender $(1976,249)]$, jedoch müßte dabei per 'Willkürakt" [Vogt (1981, 23)] das Planungsende bestimmt werden und was nach Ende des Planungshorizontes geschieht, 
würde sich auf die heutige Planung nicht auswirken ${ }^{10)}$. Jedoch gerade bei der intertemporalen Umweltallokation ist es wichtig, auch Auswirkungen in der ferneren Zukunft zu berücksichtigen.

Die Wohlfahrt der Region $i$ im Planungszeitraum $[0, \infty)$ ist demnach

$$
W_{i}=\int_{0}^{\infty} u^{i}\left[C_{i}(t), S_{i}(t)\right] e^{-\delta t} d t
$$

$\delta$ gibt hierbei die gesellschaftliche Zeitpräferenz an, die für beide Regionen gilt. Je größer $\delta$ ist, desto größer ist die Gegenwartsvorliebe der Ökonomie. Auch bei der Auswahl der sozialen Diskontrate ist ein Werturteil beinhaltet, da durch sie das Gewicht der einzelnen Generationen im Optimierungskalkül ausgedrückt wird. Wird die Diskontrate sehr niedrig gewählt, geht der Nutzen zukünftiger Generationen mit großem Gewicht in das Optimierungsproblem ein, während bei hoher Diskontrate der Nutzen der gegenwärtigen Generation stärker gewichtet wird [vgl. Siebert (1978, 150)]. Die Herleitung der sozialen Diskontrate aus den individuellen Zeitpräferenzraten ist - ähnlich dem Aggregationsproblem der sozialen Wohlfahrtsfunktion - eine "offene Frage" $[\text { Siebert }(1978,150)]^{11}$.

Diesen durch (3.10) definierten intertemporalen Wohlfahrtsfunktionen liegen einige vereinfachende Annahmen zugrunde [vgl. Vogt $(1981,21)]$ :

- Die Präferenzstruktur ist zeitlich invariant, d. h. die Wohlfahrtsfunk-

10) Arrow $(1968,92)$ begründet den unendlichen Planungshorizont für die Kapitalakkumulation folgendermaßen: "Certainly, processes of capital accumulation for the economy as a whole have no natural stopping place in the definable future. At any given future date the state of the system (...) will have implications for the further future. If we choose to stop our analysis at any fixed date, it will be necessary (...) to include in our utility functional some scrap value for the stock of capital at the end of the period. But the only logically consistent way of doing so is to determine the maximum utility attainable in the further future starting with any given stock of capital. Of course, astronomers assure us that the world as we know it will come to an end in some few billions of years. But, as elsewhere in mathematical approximations to the real world, it is frequently more convenient and more revealing to proceed to the limit to make a mathematical infinity in the model correspond to the vast futurity of the real world." Eine analoge Argumentation soll auch in dieser Arbeit bezüglich des Umweltproblems gelten.

11) Vgl. zur Problematik der Herleitung der sozialen Diskontrate z. B. Marglin (1963), Dasgupta (1969, 308 - 309), Vogt (1981, 22 - 23). 
tionen gelten für den gesamten Planungshorizont unverändert.

- Es wird Kardinalität der Wohlfahrtsfunktionen vorausgesetzt, da durch die Integration eine Addition der zeitpunktbezogene Nutzen vorgenommen wird.

- Die zeitpunktbezogenen Nutzenniveaus sind voneinander unabhängig.

\subsection{Die OPTIMIERUNGSPROBLEME UND DIE ANGEWANDTEN METHOdEN}

Es werden zwei Methoden angewandt: Einmal die Theorie der nichtkooperativen Differentialspiele bei autonomen Regionalbehörden und zum anderen die Theorie der kooperativen Differentialspiele bei einer Zentralbehörde.

Diese Theorien basieren beide auf der Kontrolltheorie. Die Theorie der Differentialspiele und die Kontrolltheorie können unter dem Begriff der "Verallgemeinerten Kontrolltheorie" subsumiert werden, wobei die Kontrolltheorie ein Spezialfall der Theorie der Differentialspiele ist ${ }^{12}$.

Die Kontrolltheorie ist eine in der ökonomischen Theorie häufig angewandte Modellform, so da $\beta$ auf eine Diskussion hier verzichtet werden $\operatorname{kann}^{13)}$.

\subsubsection{Autonome Regionalbehörden}

Eine autonome Regionalbehörde der Region $i^{14)}$ sieht sich einem intertemporalen Maximierungsproblem gegenüber:

$$
\operatorname{Max} W_{i}=\int_{0}^{\infty} u^{i}\left[C_{i}(t), S_{i}(t)\right] e^{-\delta t} d t
$$

12) Vgl. für einen Überblick über die Grundformen von Optimierungsproblemen und die "Verallgemeinerte Kontrolltheorie" Ho (1970).

13) Für eine ausführliche Darstellung der theoretischen Grundlagen vgl. z. B. Athans, Falb (1966), Bryson, Ho (1975), Hestenes (1966), Luenberger (1979), Pontryagin et al (1962). Für die für die ökonomische Anwendung aufbereitete Kontrolltheorie vgl. z. B. Arrow (1968), Arrow, Kurz (1970), Hadley, Kemp (1971), Intriligator (1971), Kamien, Schwartz (1981), Long, Vousden (1977), Takayama (1974), Toussaint (1984).

14) Unabhängig davon ob sie eine Restriktion einer übergeordneten Behörde berücksichtigen muß oder nicht. 
unter bestimmten Bedingungen ${ }^{15}$. Da durch die Definition der Bewegungsgleichungen der Zustandsvariablen $S_{i}(t)$ [vgl. Gleichung (3.4)] ein interdependentes System gegeben ist, kann die autonome Regionalbehörde der Region $i$ die Maximierung der Wohlfahrtsfunktion nicht unabhängig vornehmen, sondern muß die Rückwirkungen aus der anderen Region

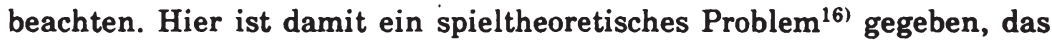
der Theorie der nichtkooperativen Differentialspiele ${ }^{17)}$ zugeordnet wird. Im folgenden wird eine auf die Fragestellungen dieser Arbeit bezogene kurze Diskussion der nichtkooperativen Differentialspiele ${ }^{18)}$ gegeben.

Ein nichtkooperatives Differentialspiel besteht aus folgenden Elementen:

- Eine Anzahl von Akteuren (zwei autonome Regionalbehörden).

- Ein Zeitintervall, das die Dauer der Entwicklung des Spieles bezeichnet $([0, T], T \rightarrow \infty)$.

- Zustandsvariablen des Systems $\left(S_{i}(t), i=1,2\right)$.

- Kontrollvariablen des Systems $\left(E_{i}(t), i=1,2\right)$.

- Differentialgleichungen(Gleichungen(3.4))

- Informationsstrukturen der Spieler. Wir betrachten im folgenden open-loop Informationsstrukturen ${ }^{19)}$, d. h. die Regionalbehörden kennen den Anfangszustand des Systems.

- Strategien der Spieler. Die Regionalbehörden richten ihre intertemporale Entscheidung über die Entwicklung ihrer Kontrollvariablen nach den ihnen zur Verfügung stehenden Informationen aus. Diese

15) Vgl. die Gleichungen (3.1) bis (3.8'), die je nach Fragestellung als Restriktionen beachtet werden müssen.

16) Einen Überblick über spieltheoretische Modelle in der Ökonomie geben Schotter, Schwödiauer (1980).

17) Die Theorie der Differentialspiele ist ein "junger" Zweig der Spieltheorie. Sie wurde begründet von Isaacs (1965), der eine dynamische Formulierung von Zwei-PersonenNull-Summen-Spielen entwickelte. Case (1967) erweiterte diese Theorie auf den Nicht-Null-Summen-Fall mit N Spielern, jedoch wurde von ihm nur ein Losungstyp vorgestellt. Eine erste allgemeine Darstellung geben Starr, Ho (1969a und b). Eine umfassende Diskussion der nichtkooperativen dynamischen Spieltheorie geben Basar, Olsder (1982).

18) Für eine rigorose Formulierung dieser Theorie vgl. Basar, Olsder (1982, 210-215 und 278-284). Darauf bauen auch die folgenden Ausführungen auf.

19) Weitere Informationsstrukturen für Differentialspiele werden in Basar, Olsder $(1982,212)$ aufgelistet. 
Entscheidungen werden Strategien der Regionalbehörden genannt.

- Der zu optimierenden Wohlfahrtsfunktionen (3.11).

Durch diese Bestandteile ist ein nichtkooperatives Differentialspiel definiert. Um eine Lösung zu erhalten, ist es noch notwendig, ein Gleichgewichtskonzept ${ }^{201} \mathrm{zu}$ definieren. Wir betrachten nichtkooperative NashGleichgewichtslösungen [vgl. Nash (1951)], die in unserer Terminologie folgendermaßen spezifiziert werden können:

Die Wohlfahrtsfunktion der Region $i$ kann geschrieben werden als

$$
W_{i}=w^{i}\left(e_{1}, e_{2}\right) \quad i=1,2
$$

wobei $e_{i}$ die Strategie der Region $i$ bedeutet ${ }^{211}$.

Ein open-loop Nash Gleichgewicht ist dann definiert als

$$
\begin{aligned}
& w^{1}\left(e_{1}{ }^{*}, e_{2}{ }^{*}\right) \geq w^{1}\left(e_{1}, e_{2}{ }^{*}\right) \\
& w^{2}\left(e_{1}{ }^{*}, e_{2}{ }^{*}\right) \geq w^{2}\left(e_{1}{ }^{*}, e_{2}\right)
\end{aligned}
$$

$e_{i}^{*}$ bezeichnet dabei die optimale Strategie der Regionalbehörde der Region $i$. Open-loop Nash-Gleichgewichte stehen in enger Beziehung zu Problemen der optimalen Kontrolle. Jede der beiden Ungleichungen in (3.13) beschreibt ein optimales Kontrollproblem, dessen Struktur nicht von den Kontrollen des jeweils anderen Akteurs beeinflußt wird [vgl. Basar, Olsder $(1982,241)]$. Für autonome Regionalbehörden bedeutet dies, daß jeweils ein Kontrollproblem zur Optimierung der Wohlfahrtsfunktion der Region gelöst werden muß.

\subsubsection{Eine Zentralbehörde ${ }^{22)}$}

Maximiert eine Zentralbehörde gemeinsam die Wohlfahrt der Gesamtökonomie, wird die Theorie der kooperativen Differentialspiele angewandt.

20) Für andere Arten von Gleichgewichtskonzepten vgl. z. B. Starr, Ho (1969a), Basar, Olsder (1982, Chapter 7).

21) Da open-loop Spiele betrachtet werden, gilt $e_{i}=E_{i}$.

22) Vgl. Leitmann (1974, 22-39). 
Bei dieser Vorgehensweise ist das sich ergebende Gleichgewicht Paretooptimal, und es kann zur Lösung des Optimierungsproblems wiederum die Kontrolltheorie angewandt werden.

Die Zentralbehörde sieht sich folgendem intertemporalen Optimierungsproblem gegenüber:

$$
\operatorname{Max} W=\sum_{i=1}^{2} \beta_{i} W_{i}
$$

unter bestimmten Bedingungen [vgl. Fußnote 15].

Es muß gelten

$$
\sum_{i=1}^{2} \beta_{i}=1
$$

$B_{i}$ ist ein Gewichtungsfaktor, mit dem die Bedeutung der Region $i$ für die Gesamtökonomie ausgedrückt wird. Bei den Betrachtungen zur Zentralbehörde wird davon ausgegangen, daß die beiden Regionen gleich gewichtet werden $\left[\beta_{i}=\frac{1}{2}, i=1,2\right]^{23)}$.

Ist das Optimierungsproblem in dieser Weise definiert, dann ist die Gleichgewichtslösung, die sich durch die Anwendung der Kontrolltheorie auf Gleichung (3.14) ergibt, Pareto-optimal [Leitmann $(1974,23)$ ].

23) Damit ist das spieltheoretische Element aus dem Optimierungsproblem im Prinzip wegdefiniert. Es ist sicherlich interessant, den Einigungsprozeß über den Anteil der Wohlfahrt einer Region an der Gesamtwohlfahrt zu untersuchen. Diese Untersuchung wird jedoch in der vorliegenden Arbeit nicht vorgenommen, sondern es wird davon ausgegangen, daß eine Zentralbehörde die Gesamtwohlfahrt des Zwei-Regionen-Systems maximiert und die beiden Regionen mit dem gleichen Gewicht in die Optimierung eingehen. Diese Vorgehensweise kann auch dahingehend interpretiert werden, da $\beta$ der Einigungsproze $\beta$ über die Gewichtung zwischen den beiden Regionen bzw. Staaten abgeschlossen ist mit dem Ergebnis, daß beide Regionen gleich gewichtet werden. 


\section{GRUNDZÜGE DER INTERTEMPORALEN UMWELTALLOKATION}

In einer ersten Annäherung an das Problem der optimalen intertemporalen Umweltallokation in einem Zwei-Regionen-System soll gezeigt werden, daß es nicht optimal sein kann, in Regionen mit unterschiedlichen regionalen Gegebenheiten identische Emissionssteuern zu setzen ${ }^{24)}$.

Um diese Fragestellung zu behandeln, gehen wir davon aus, daß die Regionen weder durch Güter- und Faktormobilität noch durch interregionale Schadstoffdiffusion miteinander verbunden sind. Dann ergibt sich, da $\beta$

$$
\begin{aligned}
& C_{i}(t)=X_{i}(t) \\
& \gamma_{i}=0
\end{aligned}
$$

\subsection{DIE OPTIMIERUNGSPROBLEME UND DIE OPTIMALBEDINGUNGEN ${ }^{25)}$}

Man erhält für jede Region $i(i=1,2)$ folgendes intertemporales Optimierungsproblem ${ }^{26)}$.

24) Diese Fragestellung knüpft an die im American Economic Review geführte Diskussion von Stein (1971), Peltzman, Tideman (1972), Tietenberg (1974) an, ob Emissionssteuern regionalisiert werden sollen.

25) Vgl. u. a. Forster (1977, 36-48), Vogt (1981, Kapitel III).

26) Hier werden autonome Regionalbehörden betrachtet. Es läßt sich aber leicht zeigen, daß sich am Ergebnis nichts ändert, wenn eine Zentralregierung die Optimierung vornimmt. 
(3.18)

$$
\begin{gathered}
\operatorname{Max} W_{i}=\int_{0}^{\infty} u^{i}\left[X_{i}(t), S_{i}(t)\right] e^{-\delta t} d t \\
\text { u.d.B. (3.1) } \quad X_{i}(t) \leq f\left[R_{i}(t), E_{i}(t)\right], \\
\text { (3.2) } \quad R_{i}(t) \geq \frac{1}{a_{i}} E_{i}(t) \\
\text { (3.3) } \quad R_{i}-R_{i}(t) \geq 0, \\
(3.4) \quad \frac{d S_{i}(t)}{d t}=E_{i}(t)-a_{i} S_{i}(t) \\
X_{i}(t), R_{i}(t), E_{i}(t) \geq 0 \forall t \in[0, \infty) \\
S_{i}(0)=S_{i}^{0} \text { gegeben } \\
\delta \text { gegeben und positiv }
\end{gathered}
$$

Für die Hamilton-Funktion in laufenden Werten erhält man ${ }^{27)}$

$$
H^{i}=u^{i}\left(X_{i}, S_{i}\right)+p_{i}\left[E_{i}-\alpha_{i} S_{i}\right]
$$

und die Lagrange-Funktion ist

$$
\begin{aligned}
L^{i}= & H^{i}+\lambda^{i}{ }_{1}\left[\dot{f}\left(R_{i}, E_{i}\right)-X_{i}\right]+\lambda^{i}{ }_{2}\left[a_{i} R_{i}-E_{i}\right] \\
& +\lambda^{i}{ }_{3}\left[R_{i}-R_{i}\right]+\lambda^{i}{ }_{4} R_{i}+\lambda^{i}{ }_{5} E_{i}+\lambda^{i}{ }_{6} X_{i}
\end{aligned}
$$

Die notwendigen und hinreichenden Bedingungen ${ }^{28}$ ) für ein Wohlfahrtsmaximum der Region $i$ sind dann:

$$
\begin{array}{ll}
L_{X}^{i}=u^{i}{ }_{X}-\lambda^{i}{ }_{1}+\lambda_{6}^{i}=0 & X_{i} \geq 0 \\
L_{R}^{i}=\lambda^{i}{ }_{1}{ }_{{ }_{R}}{ }_{R}+\lambda^{i}{ }_{2} a_{i}-\lambda^{i}{ }_{3}+\lambda^{i}{ }_{4}=0 & R_{i} \geq 0
\end{array}
$$

27) Die Variablen, die weiterhin durchweg Funktionen der Zeit sind, werden aus Vereinfachungsgründen nicht explizit als zeitabhăngig ausgewiesen, wenn sich dadurch keine Fehldeutungen ergeben.

28) Vgl. Takayama (1974, 648-650, Theorem 8.C.1 und Korollar). Daraus ergibt sich, daß die Bedingungen notwendig und hinreichend für ein Optimum sind. 


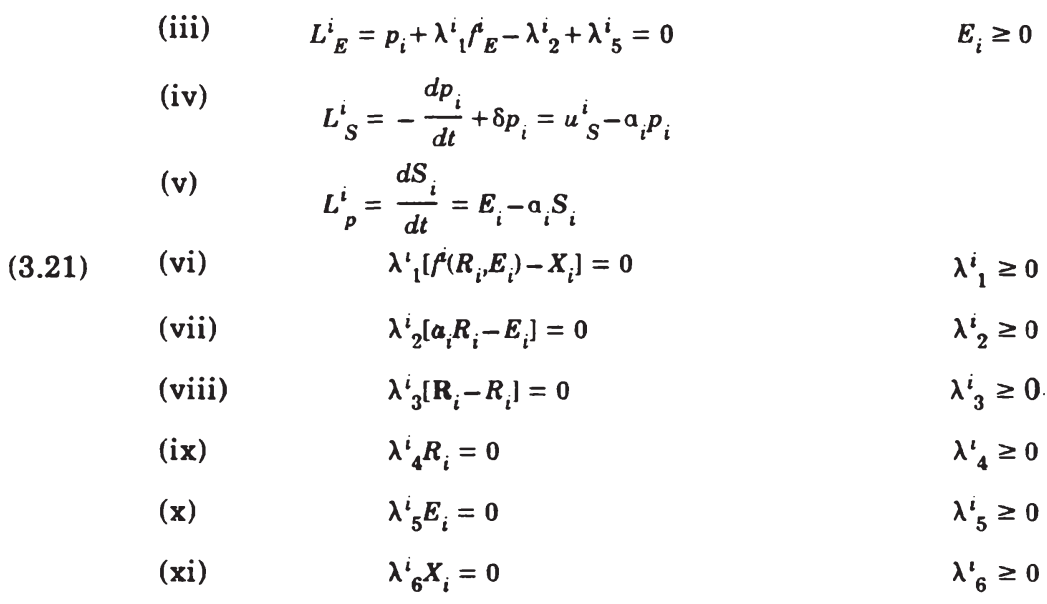

Da durch die Nutzenfunktion $u$ Nullkonsum ausgeschlossen ist, d. h. $X_{i}>$ $0 \forall t \in[0, \infty)$ ist, gilt $\lambda_{6}^{i}=0$ und deshalb

$$
\lambda_{1}^{i}=u_{X}^{i}>0
$$

Damit ist $X_{i}=f\left(R_{i}, E_{i}\right)$. Weiter gilt $R_{i}>0$ und damit $\lambda_{4}^{i}=0$, womit

$$
\lambda_{3}^{i}=\lambda_{1}^{i} f_{R}+\lambda_{2}^{i} a_{i}>0,
$$

d. h. $R_{i}=\mathbf{R}_{i}$, was Vollbeschäftigung impliziert. Sei $E_{i} \in\left(0, E_{i}^{\text {max }}\right)^{29)}$. Daraus folgt, daß $\lambda_{2}^{i}=0$ ist und deshalb $\lambda_{3}^{i}=\lambda_{1}^{i} f_{R}$. D. h., daß im Optimum der Schattenpreis des Gutes $X_{i}\left(\lambda_{1}{ }_{1}\right)$ seinen Grenzkosten entsprechen muß $\left(\lambda^{i}{ }_{3} / f_{R}\right)^{30}$.

Aus (3.21.iii) erhalten wir

$$
p_{i}=-u_{X}^{i} f_{E}^{i}+\lambda_{2}^{i}-\lambda_{5}^{i}
$$

$p_{i}$ ist hier die Kovariable des Optimierungsproblems. Sie gibt den Wert der Wohlfahrtsänderung der Region $i$ in der Zukunft an, wenn sich die Immis-

29) Es gilt $E_{i}^{\max }=a_{i} \mathbf{R}_{i}$, d. h., mehr als $E_{i}{ }^{\max }$ Emissionen können in Region $i$ nicht produziert werden.

30) Vgl. zu dieser Interpretation in statischem Kontext Siebert $(1978,40)$. 
sionen im Zeitpunkt $t$ ändern. $p_{i}$ hat also die Dimension eines Preises, und dieser Preis ist der Schattenpreis, der als Teil des Allokationsprozesses ermittelt wird, da jedes Allokationsproblem ein Bewertungsproblem beinhaltet: Der Beitrag der Zustandsvariablen zur Wohlfahrt in einem bestimmten Zeitpunkt ist der Wert der Kovariablen in diesem Zeitpunkt ${ }^{31)}$. $p_{i}$ muß hier also negativ sein, da durch die Immissionen eine Wohlfahrtssenkung eintritt.

Eine optimale Besteuerung liegt dann vor, wenn die Steuer gerade dem Beitrag der letzten Emissionseinheit zur Wohlfahrt im Zeitpunkt $t\left(u_{X}^{i} f_{E}\right)$ entspricht. Deshalb gilt

$$
\tau_{i}=u_{X}^{i} f_{E}^{i}
$$

wobei $\tau_{i}$ die optimale Steuer im Zeitpunkt $t$ angibt.

Aus (3.25) wird eine Funktion

$$
E_{i}=E^{i}\left(\tau_{i}\right)
$$

abgeleitet, die folgende Eigenschaften hat:

$$
\begin{aligned}
& E_{\tau}^{i}=\frac{1}{u_{X X}^{i}\left(f_{E}^{i}\right)^{2}+u_{X}^{i} f_{E E}^{i}}<0 \\
& \text { mit dem Definitionsbereich }\left[\tau_{i}^{i}, \tau_{i}{ }^{\prime \prime}\right] \\
& \tau_{i}^{\prime}=u_{X}^{i}{ }_{X}{ }^{i} E_{E} E_{i}=E_{i}{ }^{m a x}, \tau_{i}{ }^{\prime \prime}=u_{X}^{i}{ }_{X} f_{E} \mid E_{i}=0
\end{aligned}
$$

In diesem einfachen Fall ist dann folgende Beziehung zwischen dem Schattenpreis $p_{i}$ und der optimalen Steuer gegeben:

$$
\tau_{i}= \begin{cases}\tau_{i}{ }^{\prime} & \text { für } \lambda_{2}^{i} \geq 0 \\ -p_{i} & \text { für } \lambda_{2}^{i}=\lambda_{5}^{i}=0 \\ \tau_{i}{ }^{\prime} & \text { für } \lambda_{5}^{i} \geq 0\end{cases}
$$

31) Vgl. u. a. Arrow (1968, 87-88), Intriligator (1980, 610), Vogt (1981, 17-18 und 72). 
Schaubild 3.4 zeigt die Beziehungen zwischen den Emissionen und der Steuer einerseits sowie zwischen der Steuer und der Kovariablen andererseits und damit auch zwischen der Kovariablen und den Emissionen: Je

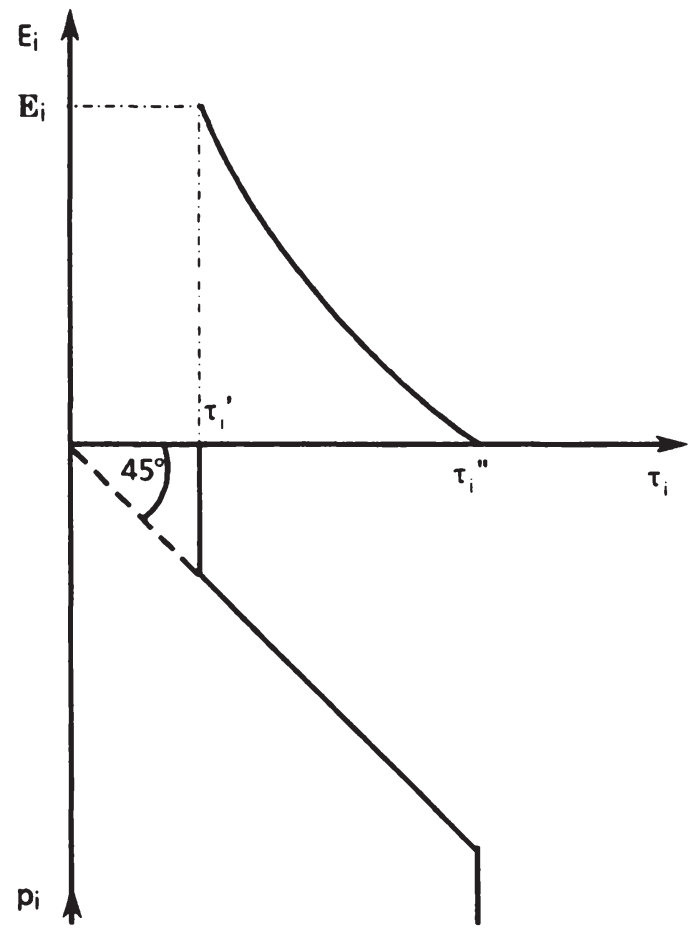

Schaubild 3.5: Die Beziehungen zwischen Emissionen, Steuern und Schattenpreis

niedriger der Zukunftsschaden durch eine zusätzliche Emissionseinheit bewertet wird $\left(p_{i}\right)$, desto niedriger ist die Steuer $\tau_{i}$, desto mehr Emissionen dürfen anfallen. Es wird also die Indifferenz bei der Bewertung zwischen heutigem Nutzenzuwachs durch eine Emissionseinheit $\left(u_{X}^{i} f_{E}\right)$ und zukünftigem Schaden durch diese Emissionseinheit $\left(-p_{i}\right)$ gefordert. 
Setzt man die Gleichungen (3.26) und (3.27) in Gleichung (3.21.iv) ein, erhält man in Verbindung mit (3.21.v) folgendes Differentialgleichungssystem $^{321}$ :

$$
\begin{aligned}
& \frac{d S_{i}}{d t}=E^{t}\left(-p_{i}\right)-a_{i} S_{i} \\
& \frac{d p_{i}}{d t}=\left(\delta+a_{i}\right) p_{i}-u_{S}^{t}
\end{aligned}
$$

Die Argumente der Funktionen sind stetig und die Funktionen sind zumindest intervallweise stetig differenzierbar, so daß nach Hadley, Kemp $(1971,370)$ für eine gegebene Anfangsverschmutzung $S_{i}{ }^{0}$ und ein geeignet gewähltes $p_{i}(0)$ eine Lösung des Differentialgleichungssystems existiert, die eindeutig ist. Diese Lösung ist ein stationäres Gleichgewicht $\left(p_{i}^{\infty}, S_{i}^{\infty}\right)$, das einen Sattelpunkt darstellt ${ }^{33)}$, wobei gilt:

$$
\begin{aligned}
& \frac{d S_{i}^{\infty}}{d t}=0, \\
& \frac{d p_{i}^{\infty}}{d t}=0 .
\end{aligned}
$$

Mit Hilfe der Phasendiagramm-Methode kann das Differentialgleichungssystem sehr anschaulich gelöst werden ${ }^{34}$. In Schaubild 3.6 ist die Ableitung des Steady-States dargestellt. Im I. Quadranten ist die stationäre Ökozustand-Funktion der Region $i$ eingezeichnet ${ }^{35)}$ :

$$
\alpha_{i} S_{i}=E_{i}
$$

32) Unter der Annahme, da $\beta E_{i} \in\left(0, E_{i}\right.$ max $)$.

33) Die Hauptminoren der Jacobischen Matrix $J$ des Systems haben im Gleichgewicht $\left(S_{2}{ }^{\infty}, p_{2}{ }^{\infty}\right)$ das gleiche negative Vorzeichen (Eindeutigkeit) [Theorem 7 in Gale, Nikaido $(1965,91)$ ]: 1. Hauptminor $=-a_{i}<0,2$. Hauptminor $=\operatorname{det} J=$ $-\alpha_{i}\left(\delta+\alpha_{i}\right)-u_{S S} E^{i} \tau<0$, d. h. die Determinante der Jacobischen Matrix $J$ ist negativ (Sattelpunkteigenschaft) [Forster (1977, 42-43)].

34) Zu dieser Methode vgl. z. B. Arrow (1968, 100 -103), Gandolfo (1980, 433 - 454).

35) Vgl. Abschnitt 3.1.2. 


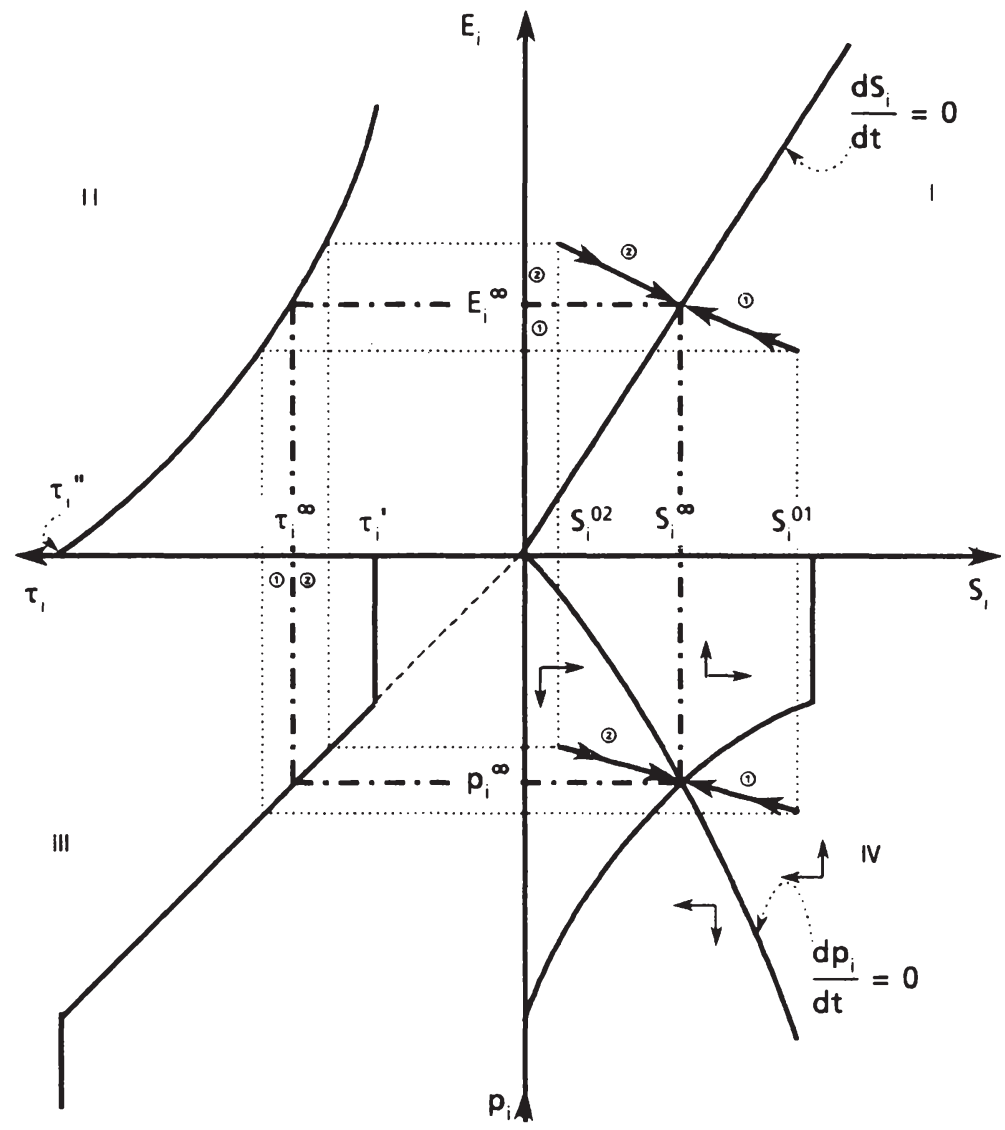

Schaubild 3.6: Die Ableitung der Trajektorien

auf der die Gleichheit der assimilationsbedingten Immissionsabnahme $\left(\alpha_{i} S_{i}\right)$ mit der emissionsbedingten Immissionszunahme $\left(E_{i}\right)$ gilt. Auch in diesem einfachen Modellzusammenhang gilt das Gesetz der Erhaltung der Masse [vgl. Kapitel 2, Gleichung (2.11'): Quellen und Abbau entsprechęn sich, während die anderen Terme alle Null sind]. 
Im I. Quadranten ist die $E^{i}\left(\tau_{i}\right)$-Funktion (3.26) dargestellt, im III. Quadranten ist Gleichung (3.27) eingetragen ${ }^{36)}$ und im IV. Quadranten ist zum einen die abgeleitete $d S_{i} / d t=0$ Kurve und zum anderen die $d p_{i} / d t=0$ Kurve eingezeichnet.

Für die $d S_{i} / d t=0$ Kurve erhalten wir

$$
S_{i}=\frac{1}{a_{i}} E^{i}\left(\tau_{i}\right) \equiv \frac{1}{a_{i}} E^{i}\left(-p_{i}\right),
$$

wobei $d S_{i} / d p_{i}=-\left(1 / \alpha_{i}\right) E^{i}{ }>0$, die $d S_{i} / d t=0$ Kurve hat im Intervall $\left(-\tau_{i}{ }^{\prime},-\tau_{i}{ }^{\prime \prime}\right)=\left(p_{i}^{\prime}, p_{i}{ }^{\prime \prime}\right)$ eine positive Steigung. Oberhalb dieser Funktion ist $d S_{i} / d t$ positiv und unterhalb negativ.

Für die $d p_{i} / d t=0$ Kurve erhalten wir

$$
p_{i}=\frac{u_{S}^{i}}{\delta+a_{i}}
$$

mit $d p_{i} / d S_{i}=u_{S S}^{i}\left(\delta+\alpha_{i}\right)<0$; die Kurve hat einen monoton fallenden Verlauf, wobei oberhalb dieser Kurve $d p_{l} / d t$ positiv ist und unterhalb negativ.

Der Schnittpunkt dieser beiden Kurven ergibt das stationäre Gleichgewicht, das Steady-State ${ }^{37 !}$.

Durch diese beiden Stationaritätsfunktionen wird der IV. Quadrant in vier Sektoren eingeteilt, in denen die Bewegungseigenschaften des Systems jeweils unterschiedlich sind.

Die Bewegungseigenschaften in der Zeit können durch Trajektorien im Phasendiagramm dargestellt werden. Es gibt unendlich viele solcher $\left(p_{i}, S_{i}\right)$-Trajektorien, die sich gemäß dem Differentialgleichungssystem (3.29) im Zeitablauf verändern, wobei die Steigung der Trajektorien durch

36) In den Quadranten III und IV ist also Schaubild 3.5 dargestellt.

37) Durch die Annahme (3.9b) ist sichergestellt, daß eine Randlösung nicht optimal sein kann, da die $d p_{d} / d t=0$-Kurve durch den Ursprung geht $\left(u_{S}^{i}=0\right.$ für $\left.S_{i}=0\right)$. Forster (1977a, 50-55) diskutiert in einem ähnlichen Modellrahmen eine Randlösung. 
$d p_{i} / d S_{i}=\left(d p_{i} / d t\right) /\left(d S_{i} / d t\right)$ gegeben ist. Daieine eindeutige Lösung existiert, ist die Optimaltrajektorie die Trajektorie, die ins Steady-State führt ${ }^{38}$. Pfade, die oberhalb des Gleichgewichtspfades liegen, verletzen eventuell die notwendigen Optimalbedingungen. Pfade, die unterhalb des Gleichgewichtspfades liegen, sind inferior [vgl. Forster $(1973,539)]$.

In Schaubild 3.6 sind nur die stabilen Äste der Trajektorien eingezeichnet, die für einen unendlichen Planungshorizont mit der Optimaltrajektorie übereinstimmen, je nachdem ob der Anfangsbestand an Immissionen kleiner oder größer als die stationäre Verschmutzung ist, obwohl es unendlich viele Trajektorien gibt, die jedoch instabil sind ${ }^{39}$. Der optimale Anfangswert des Immissionsschattenpreises der Region $i p_{i}(0)$ ist $u$. a. abhängig von der Anfangsverschmutzung in dieser Region. $p_{i}(0)$ muß c. p. umso höher (niedriger) sein, je niedriger (höher) die Anfangsverschmutzung ist.

Der Entwicklungspfad der Emissionen in Region $i$ hängt davon ab, ob die Ausgangsverschmutzung $S_{i}{ }^{0}$ größer, gleich oder kleiner der stationären Immission $S_{i}^{\infty}$ ist: Aus den Gleichungen (3.26), (3.27) und (3.28) erhält $\operatorname{man}$

$$
\frac{d E_{i}}{d t}=-E_{\tau}^{i} \frac{d p_{i}}{d t} \quad \begin{cases}>0 & \text { für } S_{i}{ }^{0}>S_{i}^{\infty} \\ =0 & \text { für } S_{i}{ }^{0}=S_{i}^{\infty} \\ <0 & \text { für } S_{i}{ }^{0}<S_{i}^{\infty}\end{cases}
$$

Aus Schaubild 3.6 erkennt man, daß für $S_{i}{ }^{0}=S_{i}{ }^{01}>S_{i}{ }^{\infty}$ der Immissionsschattenpreis $p_{i}(0)$ so niedrig gesetzt wird - und damit die Steuer $\tau_{i}$ so hoch - , daß die Emissionen relativ niedrig sind $\left(E_{i}{ }^{01}\right)$, d. h. die Güterproduktion ist relativ klein. Durch diese niedrigen Emissionen verbessert sich die Umweltqualität im Zeitablauf, d. h. die Immission nimmt ab. Dadurch kann wieder mehr emittiert werden, die Produktion in Region $i$

38) Dies gilt für unendlichen Planungshorizont. Zur weiteren Ableitung der Optimaltrajektorien vgl. auch Vogt (1981, Textziffern III 19 und 20).

39) Dies folgt aus der Eindeutigkeit der Lösung, d. h. die Trajektorien schneiden sich nicht. 
wächst im Zeitablauf mit steigendem $p_{i}$. Dieser Prozeß kommt zum Stillstand, wenn das stationäre Gleichgewicht $\left(S_{i}^{\infty}, p_{i}^{\infty}\right)$ erreicht ist [vgl. die Pfeile (1) in Schaubild 3.6]. Ist andererseits $S_{i}{ }^{0}=S_{i}{ }^{02}<S_{i}{ }^{\infty}$, ergeben sich die analogen Anpassungen [vgl. die Pfeile (2) in Schaubild 3.6].

Setzt die Wirtschaftspolitik falsche Signale, indem sie die Steuer $\tau_{i}(0) z u$ niedrig bzw. zu hoch setzt, sind die Emissionen im gesamten Planungszeitraum zu hoch bzw. zu niedrig und der Schattenpreis steigt (sinkt) im Zeitablauf, d. h. die Steuer sinkt (steigt). Solche Trajektorien erfüllen nicht im gesamten Planungshorizont die Optimalbedingungen für innere Lösungen, sondern es wird entweder der Immissionsbestand Null oder die Kuppelproduktionsgrenze wird voll ausgenutzt. Folgendes Schaubild zeigt beispielhaft solche ineffizienten Trajektorien. Wegen der Sattelpunkteigen-

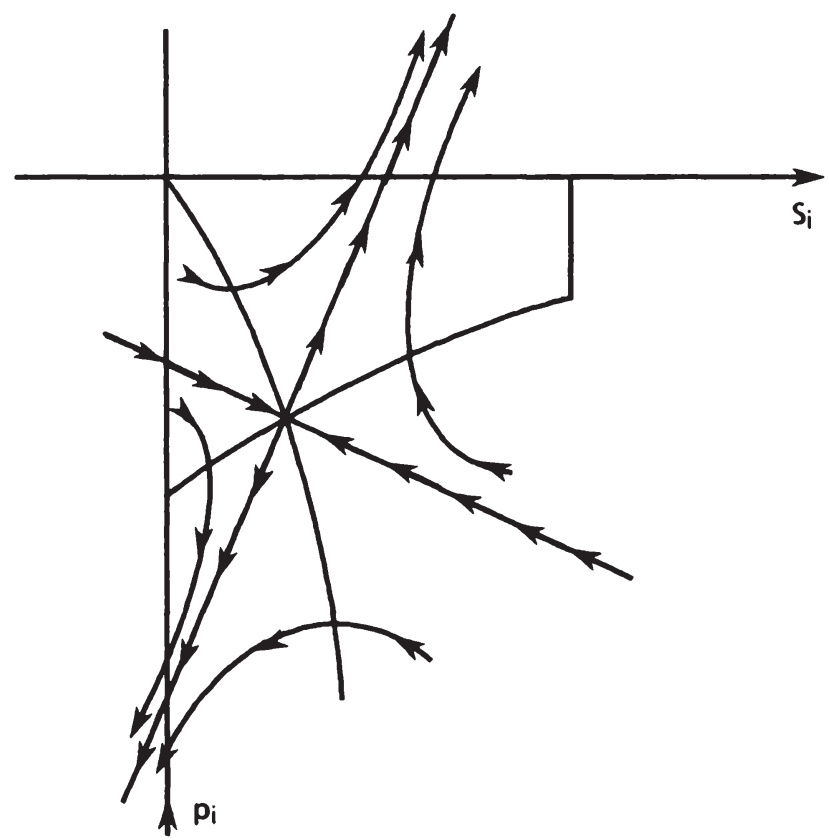

Schaubild 3.7: Unbeschränkte Trajektorien 
schaft der Optimallösung, gibt es zwei Trajektorien die ins Steady-State führen, während zwei Trajektorien daraus weg führen. Diese Trajektorien sind Asymptoten für alle anderen Trajektorien, für die ein anderer Anfangswert von $p_{i}$ als der optimale gewählt wurde. Diese allgemeine Aussage gilt für die unbeschränkte Optimierung, $d$. h. für die Variablen ist keine Nichtnegativitätsbedingung zu beachten [vgl. hierzu Gandolfo (1980, 435-436)]. Da für eine ökonomisch sinnvolle Lösung diese Nichtnegativitätsbedingungen der Variablen berücksichtigt werden müssen, müssen die Schattenpreise für die Vollausnutzung der Restriktion bei den Ableitungen der Trajektorien berücksichtigt werden. Aus dem Differentialgleichungssystem (3.28) wird dann

$$
\begin{aligned}
& \frac{d S_{i}}{d t}=E^{i}\left(-p_{i}-\lambda_{2}^{i}+\lambda_{5}^{i}\right)-a_{i} S_{i} \\
& \frac{d p_{i}}{d t}=\left(\delta+a_{i}\right) p_{i}-u_{S}^{i}
\end{aligned}
$$

Betrachten wir zuerst den Fall, daß die Steuer höher gesetzt wird als optimal ist:

$$
\tau_{i}{ }^{h}(0)>\tau_{i}{ }^{o p t}(0) \Leftrightarrow p_{i}{ }^{h}(0)<p_{i}^{o p t}(0),
$$

d. h. die Region befindet sich auf einer Trajektorie, die unterhalb der Optimaltrajektorien verläuft. Solange $E_{i}>0$ gilt, sind die Schattenpreise $\lambda_{2}^{i}=\lambda_{5}^{i}=0$. Wird jedoch $E_{i}=0$ in einem Zeitpunkt $t$, kann $\lambda_{5}^{i}>0$ gelten. $p_{i}$ ist in diesem Fall sehr niedrig (betragsmäßig hoch). $E_{i}$ wird jedoch nicht negativ, so daß $d S_{i} / d t=-\alpha_{i} S_{i}$ gilt und damit $S_{i}$ mit der konstanten Rate $\alpha_{i}$ sinkt, jedoch nie Null wird. Damit ist $u_{S}^{i}<0 \forall t \in[0, \infty)$. Es gilt aber $\left(\delta+\alpha_{i}\right) p_{i}<u_{S}^{i}$ und damit $d p_{i} / d t<0$. Damit sinkt aber der Schattenpreis immer weiter bis unendlich. Dies kann also nicht optimal sein.

Betrachten wir den Fall, daß die Emissionen nicht alle entsorgt werden können, wenn produziert wird. Dies wird ausgedrückt durch

$$
f_{E}\left(R_{i}, 0\right)=\infty \text {. }
$$


Dann gibt es kein endliches $\tau_{i}$, für das $E_{i}=0$ wird, d. h. für $X_{i}>0$ ist auch immer $E_{i}>0$. Mit zu hoher Steuer geht die Produktion des Gutes $X$ im Zeitablauf gegen Null. Dies widerspricht jedoch den Annahmen über die Wohlfahrtsfunktion, so daß keine Optimallösung erreicht wird.

Ist andererseits die Steuer im Anfangszeitpunkt zu niedrig, werden zu viele Emissionen und zu viele Güter produziert. Im gesamten Zeitablauf verläuft diese Trajektorie oberhalb der Optimaltrajektorie. Die Emissionen steigen solange, bis die Kuppelproduktionsgrenze voll ausgenutzt wird. Die Emissionen können nicht weiter steigen, sondern verbleiben bei $E_{i}=E_{i}{ }^{\text {max }}$. Damit gibt es dann einen Zeitpunkt $t$, für den $p_{i}=0$ gilt. Es gilt aber weiterhin $d p_{i} / d t=-u_{s}^{i}>0$. Der Schattenpreis wird positiv. Da $S_{i}$ höchstens $S_{i}{ }^{\max }$ werden kann, wird $u_{S}^{i}$ zu einer Konstanten ab einem bestimmten Zeitpunkt. Es gilt dann

$$
\frac{d p_{i}}{d t}=\left(\delta+a_{i}\right) p_{i}-u_{S}^{i}>0
$$

und zwar steigt der Schattenpreis immer stärker, da er positiv ist. Dieses so dargestellte Scenario widerspricht aber der Interpretation der Kovariablen, die den Wohlfahrtsbeitrag einer Immissionseinheit ausdrückt und eine Immissionseinheit wird annahmegemäß negativ für die Wohlfahrt bewertet.

Mit den obigen Ausführungen wurde gezeigt, daß nur die Trajektorien, die ins Steady-State führen, optimal sind. Deshalb werden sich die weiteren Ausführungen in dieser Arbeit auf Fälle beschränken, die optimal in obigem Sinne sind. Es wird immer davon ausgegangen, daß die Wirtschaftspolitik die "richtigen" Steuern setzt, so daß sich die Ökonomie langfristig zu einem Steady-State hin bewegt, oder wie es Forster $(1973,539)$ ausdrückt: "the control models discussed here (...) are non-stochastic. There is no reason for the economy to get off an optimal trajectory in such a controlled system. The system is strictly stable subject to the optimal control". 
Die soziale Diskontrate $\delta$ gibt die Gegenwartsvorliebe der Ökonomie an. Je größer $\delta$ ist, mit umso geringerem Gewicht geht die zukünftige Wohlfahrt in das Kalkül der Planer ein. Zu fragen ist jetzt, welche Auswirkungen auf das Steady-State unterschiedlich hohe Diskontraten haben. Die Analyse erfolgt durch parametrische Variation von $\delta$ und die Betrachtung der Auswirkungen auf die Steady-State-Werte der Variablen ${ }^{40}$. Es treten die zu erwartenden Ergebnisse ein: Je größer $\delta$ ist, umso größer sind die Steady-State-Emissionen - und damit auch die Produktion des Konsumgutes -, die Immissionen und desto kleiner ist der bewertete Zukunftsschaden und die zu setzende Steuer. Das sich ergebende Steady-State ist also entscheidend mit von der Gegenwartsvorliebe der Ökonomie abhängig. Je kleiner die Gegenwartsvorliebe der Ökonomie ist, desto mehr wird auf die Umweltqualität geachtet, $d$. h. desto geringer sind die Immissionen dadurch, daß vermehrt Faktoren in die sektorinterne Entsorgung eingesetzt werden.

Die jetzt untersuchte Frage ist, ob es optimal sein kann, identische Steuern festzulegen, wenn die Regionen unterschiedlich sind. Unterschiede können einmal technischer Natur sein, wie z. B. unterschiedliche Produktionstechnologien, unterschiedlich große Faktorausstattung; oder aus den Gegebenheiten des Ökosystems entspringen, z. B. kann die Assimilationsfähigkeit des Ökosystems in einer Region größer sein als in der anderen; weiter kann die Bewertung des Umweltschadens unterschiedlich sein, dies würde sich in unterschiedlichen Nutzenfunktionen ausdrücken.

Wir wollen hier zwei Fälle untersuchen: einmal, daß die Assimilationsfähigkeit des Ökosystems in den Regionen unterschiedlich ist:

40) Für die analytische Ableitung vgl. den Anhang zu Kapitel 3.

41) Die in diesem Abschnitt vorgenommene Analyse unterschiedlicher Faktorausstattungen und Assimilationsparameter in den Regionen kann auch dahingehend interpretiert werden, daß für eine Region die Auswirkungen der Größe dieser Parameter auf das Steady-State untersucht werden. Nach diesem Muster wird in den folgenden Kapiteln 4, 5 und 6 verfahren. 


$$
\alpha_{1}>\alpha_{2}
$$

und zum anderen, daß die Faktorausstattungen unterschiedlich sind:

$$
\mathbf{R}_{1}>\mathbf{R}_{2}
$$

Ansonsten seien die Regionen identisch.

Gelte zuerst Bedingung (3.34), in Region 1 ist die Assimilationsfähigkeit des Ökosystems größer als in Region 2. In Region 1 kann also für den gleichen Immissionsbestand mehr emittiert werden als in Region 2.

Es gilt

$$
E^{1}\left(\tau_{1}\right)=E^{2}\left(\tau_{2}\right)
$$

da diese Funktionen Gegebenheiten ausdrücken, die unabhängig sind von $\alpha_{i}$. In folgendem Schaubild 3.8 ist diese Situation dargestellt.

Als erstes sehen wir, daß die stationäre Ökozustand-Funktion für Region 1 steiler verläuft als für Region 2 (I. Quadrant). Im II. Quadranten ist die $E^{i}$ Funktion, die für beide Regionen identisch ist, eingezeichnet. Im III. Quadranten ist wieder die Beziehung zwischen Zukunftsschaden und Steuer eingezeichnet und im IV. Quadranten sind einmal die abgeleiteten $d S_{i} / d t=0$ Kurven eingezeichnet. Diese Kurven haben für $p_{i} \leq p_{i}{ }^{\prime \prime}$ (die Immissionen sind Null) einen identischen Ast. Da dies jedoch eine Randlösung ist, betrachten wir diesen Fall nicht weiter. Für $p_{i}>p_{i}{ }^{\prime \prime}(i=1,2)$ verläuft die $d S_{1} / d t=0$ Kurve links von der $d S_{2} / d t=0$ Kurve. Die maximale Immission, die in Region 1 auftreten kann, ist kleiner als diejenige in Region $2\left(S_{1}^{\max }<S_{2}^{\max }\right)$.

Betrachten wir jetzt die $d p_{i} / d t=0$ Kurve. Es gilt Gleichung (3.32)

$$
p_{i}=\frac{u_{S}^{i}}{\delta+a_{i}}
$$

Ist $S_{1}=S_{2}>0$, dann sind auch die Grenznutzen gleich $\left(u_{S}^{1}=u^{2}{ }_{S}\right)$, wegen der Separabilität der Nutzenfunktion. Es folgt 


$$
\left(\delta+\alpha_{1}\right) p_{1}=\left(\delta+\alpha_{2}\right) p_{2}
$$

und deshalb

$$
p_{1}>p_{2} \quad \Leftrightarrow \quad \tau_{1}<\tau_{2} .
$$

Sind die Immissionen in beiden Regionen gleich groß, ist es optimal, in Region 1 eine niedrigere Steuer zu setzen als in Region 2.

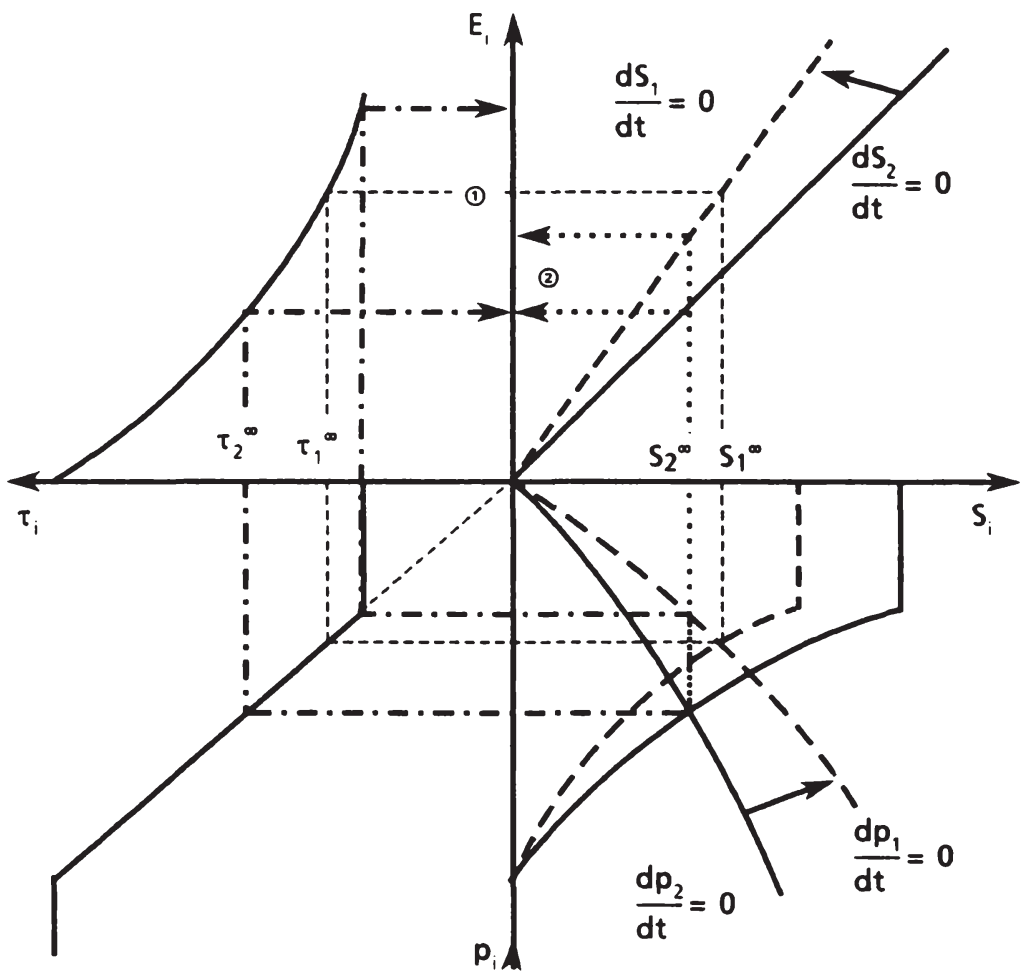

Schaubild 3.8: Unterschiedliche Assimilationsfähigkeiten 
Für beide Steady-States gilt im Vergleich, daß die Steuer in Region $1 \mathrm{im}$ Optimum niedriger ist als in Region 2. Daraus folgt aber auch, daß in Region 1 mehr von dem Konsumgut $X$ produziert wird, da die Emissionen in Region 1 größer sind als in Region 2.

Über das Verhältnis der optimalen Schadstoffbestände zueinander kann keine eindeutige Aussage gemacht werden. Der optimale Immissionsbestand in Region 1 kann sowohl größer als auch kleiner oder gleich dem in Region 2 sein. Dies hängt von der Gegenwartsvorliebe der Ökonomie ab, also von der sozialen Diskontrate $\delta$, die die Steigung der $d p_{i} / d t=0$ Kurven mitbestimmt ${ }^{42}$. Je größer die Gegenwartsvorliebe in der Ökonomie ist, desto niedriger ist der Einfluß der Assimilationsfähigkeit des Ökosystems $\alpha_{i}$ und desto näher liegen die stationären Kovariablen-Funktionen beieinander, da für die Regionen der heutige Konsum relativ wichtiger ist als die morgige Verschmutzung und damit der Unterschied der Assimilationsfähigkeit der Ökosysteme etwas zurücktritt. Es ist dann zu erwarten, daß der optimale Immissionsbestand in Region 2 größer ist als in Region 1. Ist andererseits die Diskontrate $\delta$ niedrig, $d$. h. die Zukunft wird stärker berücksichtigt, verlaufen die $d p_{i} / d t=0$ Kurven steiler und man kann erwarten, daß der Steady-State-Immissionsbestand in Region 1 größer ist als in Region 2, da durch die stärkere Zukunftsberücksichtigung in der Ökonomie in Region 2 die Emissionen stärker vermindert werden, da die unterschiedlichen Assimilationsfähigkeiten der Ökosysteme einen stärkeren Einfluß auf die Steigung der $d p_{i} / d t=0$ Kurven ausüben ${ }^{43)}$.

Exkurs: Betrachten wir eine Region bei Variation des Assimilationskoeffizienten $\alpha_{i}$. Die Änderungsrichtung der Immissionen hängt von folgender Beziehung ab

$$
\left.\frac{d E_{i}}{d \tau_{i}} \frac{d \tau_{i}}{d p_{i}} \frac{d p_{i}}{d a_{i}}\right|_{d S_{i}=0}-\left.\frac{d E_{i}}{d a_{i}}\right|_{d S_{i}=0} \geq 0
$$

42) Vgl. hierzu auch für Ein-Region-Modelle Forster $(1977,48)$, Siebert $(1978,177$ 178), Vogt (1981, 80 - 81).

43) Im Anhang sind diese Ergebnisse modelltheoretisch abgeleitet. 
Ist (3.36) positiv, dann steigen die Steady-State-Immissionen $^{44)}$ während sie für negatives (3.36) sinken. Man hat zwei Effekte, die sich gegenüberstehen: Effekt 1 kann als Emissions-Steuer-Effekt bezeichnet werden; er gibt die Auswirkungen einer Änderung des Assimilationskoeffizienten an, die über die Steuer $\tau_{i}$ auf die Emissionen wirken (bei konstanten Immissionen). Effekt 2 ist der reine Emissionseffekt, der die Auswirkungen einer Änderung von $\alpha_{i}$ angibt, die direkt über das Ökosystem wirken (bei konstanten Immissionen).

Betrachten wir jetzt Bedingung (3.35). In Region 1 ist die Faktorausstattung größer als in Region 2. Hier ändert sich die $E^{i}\left(\tau_{i}\right)$-Funktion und zwar ist für $\tau_{1}=\tau_{2}=\tau E^{1}(\tau)>E^{2}(\tau)$. Anhand des folgenden Schaubildes 3.9 wird die Analyse vorgenommen. Im I. Quadranten ist die stationäre Ökozustand-Funktion, die für beide Regionen gilt, eingezeichnet. Im I. Quadranten sind die beiden $E^{i}\left(\tau_{i}\right)$-Funktionen eingezeichnet. Im III. Quadranten ist die Beziehung zwischen den Schattenpreisen und den Steuern dargestellt und im IV. Quadranten die $d S_{i} / d t=0$ und die $d p_{i} / d t=0$ Kurven. Die $d S_{1} / d t=0$ Kurve verläuft rechts von der $d S_{2} / d t=0$ Kurve und da die $d p_{i} / d t=0$ Kurve für beide Regionen gilt, ist $S_{1}{ }^{\infty}>S_{2}{ }^{\infty}$ und auch $\tau_{1}{ }^{\infty}>$ $\tau_{2}{ }^{\infty}$, d. h. die optimale Steuer in Region 1 ist größer als in Region 2. Durch den größeren Ressourcenbestand in Region 1 kann dort mehr produziert werden als in Region 2.

44) Dieser Fall ist in Schaubild 3.8 dargestellt. 


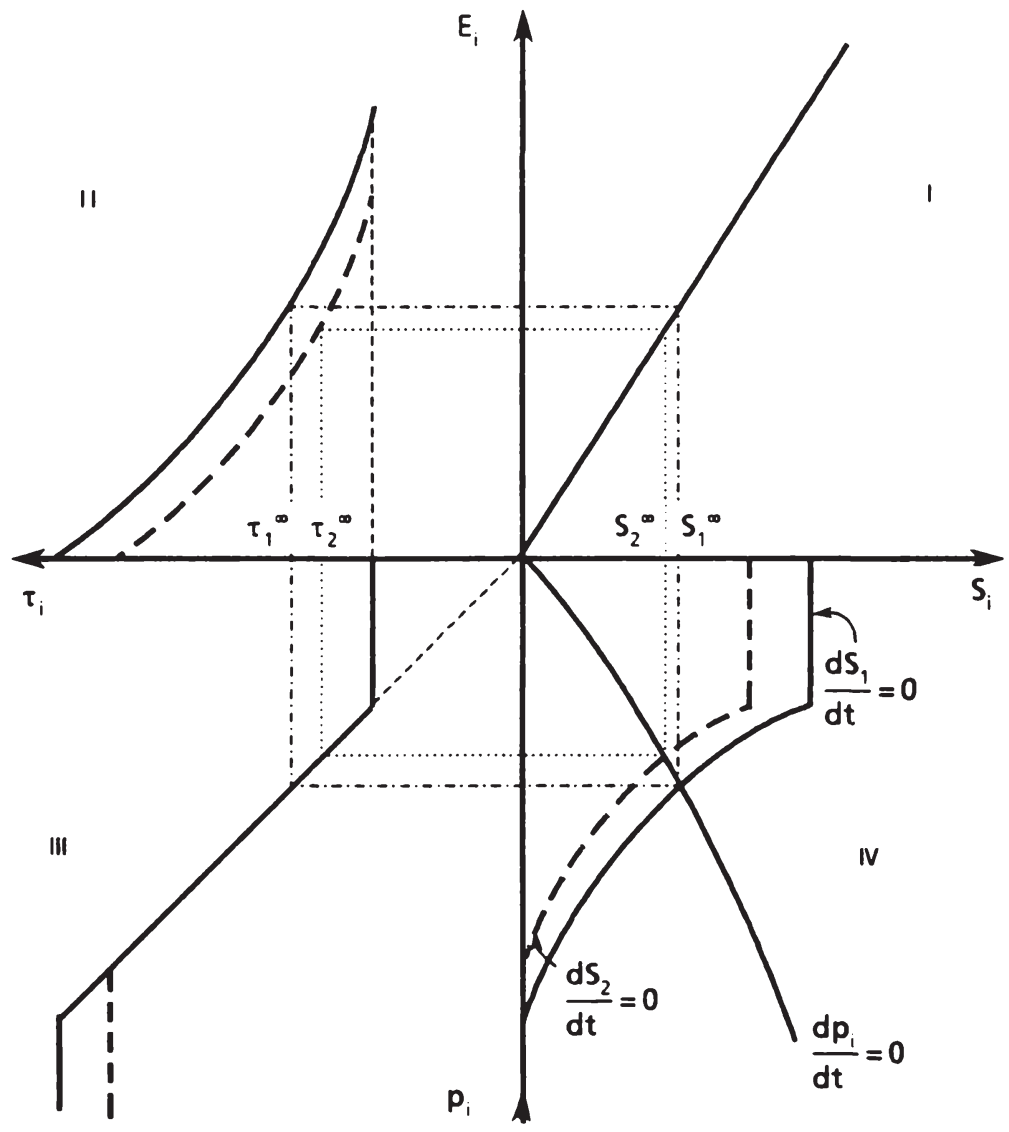

Schaubild 3.9: Unterschiedliche Faktorausstattung ${ }^{45)}$

45) Hier wurde davon ausgegangen, daß $\tau_{1}^{\prime}=\tau^{\prime}{ }_{2}$. Darüber kann jedoch keine Aussage gemacht werden, da $\tau_{1}^{\prime}$ sowohl größer als auch kleiner $\tau^{\prime}{ }_{2}$ sein kann. Es werden jedoch keine Randlösungen betrachtet, so daß die Grenzen für uns unerheblich sind. 


\section{ZUSAMMENFASSUNG}

Nach einer Vorstellung der Modellgleichungen wird anhand eines intertemporalen neoklassischen Optimierungsmodells gezeigt, daß es in einer Zwei-Regionen-Ökonomie nicht optimal sein kann, identische Emissionssteuern zu erheben, wenn sich die Regionen z. B. bei der Assimilationsfähigkeit des Ökosystems oder in der Faktorausstattung unterscheiden. Es wird gezeigt, daß in der Region,

(i) in der die Assimilationsfähigkeit des Ökosystems größer ist, niedrigere Emissionssteuern zu erheben sind, während die Produktion - und damit die Emissionen - dort größer sind als in der Region mit niedrigerem Assimilationskoeffizienten (allerdings kann in der Region mit höherem Assimilationskoeffizienten ein höherer Immissionsbestand auftreten);

(ii) in der der Faktorbestand höher ist, höhere Emissionssteuern festzusetzen sind, da dort mehr produziert wird - sowohl Konsumgüter als auch Emissionen - und damit die Immissionen steigen.

Unterscheiden sich die Regionen sowohl in der Faktorausstattung als auch in der Assimilationsfähigkeit ihres Ökosystems, können identische Emissionssteuern in beiden Regionen nur ein Zufallsergebnis sein, wie obige Ausführungen zeigen.

Des weiteren wird untersucht, welche Auswirkungen die Höhe der sozialen Zeitpräferenzrate auf das Steady-State der Ökonomie hat. Je höher die soziale Diskontrate ist, desto stärker geht der Nutzen der gegenwärtigen Generation in das Optimierungskalkül ein. Damit ist der direkte positive Nutzeneffekt des Konsums wichtiger als der indirekte, in der Zukunft liegende negative Nutzeneffekt der Immissionen, so daß die Konsumgüterproduktion größer wird.

Die in diesem Kapitel erzielten Ergebnisse sind Grundlage der Analyse in den weiteren Kapiteln, auf die immer wieder zurückgegriffen wird. 


\section{Anhang 3}

Totale Differentiation des Gleichungssystems

$$
\begin{aligned}
E_{i}-\alpha_{i} S_{i} & =0 \\
\left(\delta+\alpha_{i}\right) p_{i}-u_{S}^{i} & =0 \\
\tau_{i}+p_{i} & =0 \\
\tau_{i}-E^{i}\left(\tau_{i}\right) & =0
\end{aligned}
$$

führt zu der Matrixgleichung

$$
\left[\begin{array}{cccc}
1 & -\alpha_{i} & 0 & 0 \\
0 & -u_{S S}^{i} & \delta+\alpha_{i} & 0 \\
0 & 0 & 1 & 1 \\
1 & 0 & 0 & -E_{\tau}^{i}
\end{array}\right]\left[\begin{array}{c}
d E_{i} \\
d S_{i} \\
d p_{i} \\
d \tau_{i}
\end{array}\right]=\left[\begin{array}{c}
S_{i} \\
-p_{i} \\
0 \\
0
\end{array}\right] d \alpha_{i}+\left[\begin{array}{c}
0 \\
-p_{i} \\
0 \\
0
\end{array}\right] d \delta+\left[\begin{array}{c}
0 \\
0 \\
0 \\
E_{\mathbf{R}}^{i}
\end{array}\right] d \mathbf{R}_{i}
$$

wobei $\quad E_{\mathbf{R}}^{t}=-\frac{u_{X X}^{i} f_{E}^{i} f_{\mathbf{R}}^{d}+u_{X}^{t} f_{E \mathbf{R}}^{i}}{u_{X X}^{i}\left(f_{E}^{i}\right)^{2}+u_{X}^{i} f_{E E}^{i}}$

Die Systemdeterminante ist

$$
\Delta_{i}=\alpha_{i}\left(\delta+\alpha_{i}\right)+u_{S S}^{i} E_{\tau}^{i}>0
$$

1. Die Effekte einer exogenen Änderung des Assimilationskoeffizienten $\alpha_{i}$ :

$$
\begin{aligned}
& \frac{d E_{i}}{d a_{i}}=\frac{E_{\tau}^{i}\left(a_{i} p_{i}+u_{S S}^{i} S_{i}\right)}{\Delta_{i}}>0 \\
& \frac{d S_{i}}{d a_{i}}=\frac{E_{i}^{i} p_{i}-\left(\delta+a_{i}\right) S_{i}}{\Delta_{i}} \geq 0 \Leftrightarrow \frac{E_{t}^{i} p_{i}}{\delta+a_{i}} \geq S_{i}
\end{aligned}
$$




$$
\begin{aligned}
& \frac{d p_{i}}{d \mathrm{a}_{i}}=-\frac{a_{i} p_{i}+u_{S S}^{i} S_{i}}{\Delta_{i}}>0 \\
& \frac{d \tau_{i}}{d \mathrm{a}_{i}}=\frac{a_{i} p_{i}+u_{S S}^{i} S_{i}}{\Delta_{i}}<0
\end{aligned}
$$

2. Die Effekte einer exogenen Änderung der sozialen Diskontrate $\delta$

$$
\begin{aligned}
& \frac{d E_{i}}{d \delta}=\frac{E_{i}^{i} a_{i} p_{i}}{\Delta_{i}}>0 \\
& \frac{d S_{i}}{d \delta}=\frac{E_{\tau}^{i} p_{i}}{\Delta_{i}}>0 \\
& \frac{d p_{i}}{d \delta}=-\frac{a_{i} p_{i}}{\Delta_{i}}>0 \\
& \frac{d \tau_{i}}{d \delta}=\frac{a_{i} p_{i}}{\Delta_{i}}<0
\end{aligned}
$$

3. Die Effekte einer exogenen Erhöhung des Faktorbestandes $\mathbf{R}_{i}$

Hier ist zuerst das Vorzeichen von $E^{i}{ }_{R}$ zu betrachten: Das Vorzeichen hängt von $u_{X X}^{i} f_{E}^{i} f_{R}+u_{X}^{i} f_{E R}\left\{>={ }_{<}\right\} 0 \mathrm{ab}$.

Aus den Annahmen über die Nutzenfunktionen folgt

$$
-\frac{u_{X X}^{i}}{u_{X}^{i}} X_{i}=\eta^{i}<1
$$

Wegen der $k$-Homogenität der Produktionsfunktionen bezüglich dem Faktor $R_{i}$ gilt: 
(i)

$$
P_{R} R_{i}=k X_{i}
$$

(ii)

$$
f_{E R}^{i} R_{i}=k f_{E}^{i}
$$

Deshalb gilt

$$
\frac{f_{E R}^{i}}{f_{E}^{i} f_{R}^{\prime}} X_{i}=1
$$

Einsetzen ergibt

$$
\frac{f_{E}^{i} f_{R}^{i} u_{X}^{i}}{X_{i}}\left(\frac{f_{E R}^{i}}{f_{E}^{i} f_{R}^{i}} X_{i}+\frac{u_{X X}^{l}}{u_{X}^{i}} X_{i}\right)=\frac{f_{E}^{c} f_{R}^{i} u_{X}^{i}}{X_{i}}\left(1-\eta^{i}\right)>0
$$

Und deshalb ist

$$
E_{\mathrm{R}}^{t}>0^{46)}
$$

Dann ergibt sich

$$
\begin{aligned}
& \frac{d E_{i}}{d \mathbf{R}_{i}}=\frac{E_{\mathbf{R}}^{i}\left(\delta+a_{i}\right) a_{i}}{\Delta_{i}}>0 \\
& \frac{d S_{i}}{d \mathbf{R}_{i}}=\frac{E_{\mathbf{R}}^{i}\left(\delta+a_{i}\right)}{\Delta_{i}}>0 \\
& \frac{d p_{i}}{d \mathbf{R}_{i}}=\frac{E_{\mathbf{R}}^{i} u_{S S}^{i}}{\Delta_{i}}<0 \\
& \frac{d \tau_{i}}{d \mathbf{R}_{i}}=-\frac{E_{\mathbf{R}^{i}}^{i} S S}{\Delta_{i}}>0
\end{aligned}
$$

46) Beachte den Unterschied zu Vogt $(1981,100$ - 102), der davon ausgeht, daß vermehrter Ressourceneinsatz die Grenzproduktivităt der Emissionen nicht beeinflußt, d. $h$. in unserer Terminologie $f_{E R}=0$, so daß in diesem Fall $E_{R}^{i}<0$ folgt. 
4. Die Effekte sind in folgender Tabelle zusammengefaßt

\begin{tabular}{|c|ccccc|}
\hline & $d E_{i}$ & $d S_{i}$ & $d p_{i}$ & $d \tau_{i}$ & $d X_{i}$ \\
\hline$d \delta$ & $\oplus$ & $\oplus$ & $\oplus$ & $\ominus$ & $\oplus$ \\
$d \alpha_{i}$ & $\oplus$ & $?$ & $\oplus$ & $\ominus$ & $\oplus$ \\
$d \mathbf{R}_{i}$ & $\oplus$ & $\oplus$ & $\ominus$ & $\oplus$ & $\oplus$ \\
\hline
\end{tabular}

Tabelle 3.1: Die Effekte von Parameteränderungen 


\section{Kapitel 4: Interregionale Verflechtung durch Diffusion}

\section{Problemstellung}

Die intertemporale Umweltallokation wurde bisher hauptsächlich "in a wonderland of no other decision units" [Nijkamp $(1978,154)]$ untersuch $t^{11}$, d. h. spill-over Effekte wurden per Definition ausgeschlossen. Es wurde also davon ausgegangen, daß es nur einen Entscheidungsbefugten oder zumindest nur eine Zielfunktion gibt [Kydland $(1975,321)]$. In der in diesem Kapitel vorgenommenen Analyse wird deshalb angenommen, daß eine Region (Region 2) durch Diffusionsvorgänge das Ökosystem der anderen Region (Region 1) beeinflußt ${ }^{2}$, und es werden drei unterschiedliche institutionelle Arrangements untersucht.

In Abschnitt 2 werden autonome Regionalregierungen betrachtet, $d$. h. es wird der klassisch-föderalistische Fall untersucht [Frey (1977)]. In Abschnitt 3 wird eine Zentralregierung betrachtet, die die Gesamtwohlfahrt der Ökonomie unter Berücksichtigung von regionenspezifischen Eigenschaften maximiert und im 4. Abschnitt wird untersucht, welche Auswirkungen sich ergeben, wenn die Zentralregierung nicht die Gesamtwohlfahrt maximiert, sondern über die Setzung eines Emissionsstandards für die emissionsexportierende Region in die Optimierung der Regionen eingreift.

Für alle Modellvarianten in diesem Kapitel gilt die folgende Spezifizierung der Ökozustand-Funktionen [vgl. Gleichung (3.4)]:

$$
\frac{d S_{1}}{d t}=E_{1}+Y_{2} E_{2}-a_{1} S_{1}
$$

(ii)

$$
\frac{d S_{2}}{d t}=\left(1-\mathrm{Y}_{2}\right) E_{2}-\mathrm{a}_{2} S_{2}
$$

1) Vgl. z. B. d'Arge, Kogiku (1973), Comolly (1975), Converse (1974), Fisher et al (1972), Forster (1973 und 1977b), Keeler et al (1972), Mäler (1974), Vogt (1981), Wright (1974).

2) Die Struktur der Ökonomie ist in Schaubild 4.1 dargestellt. 


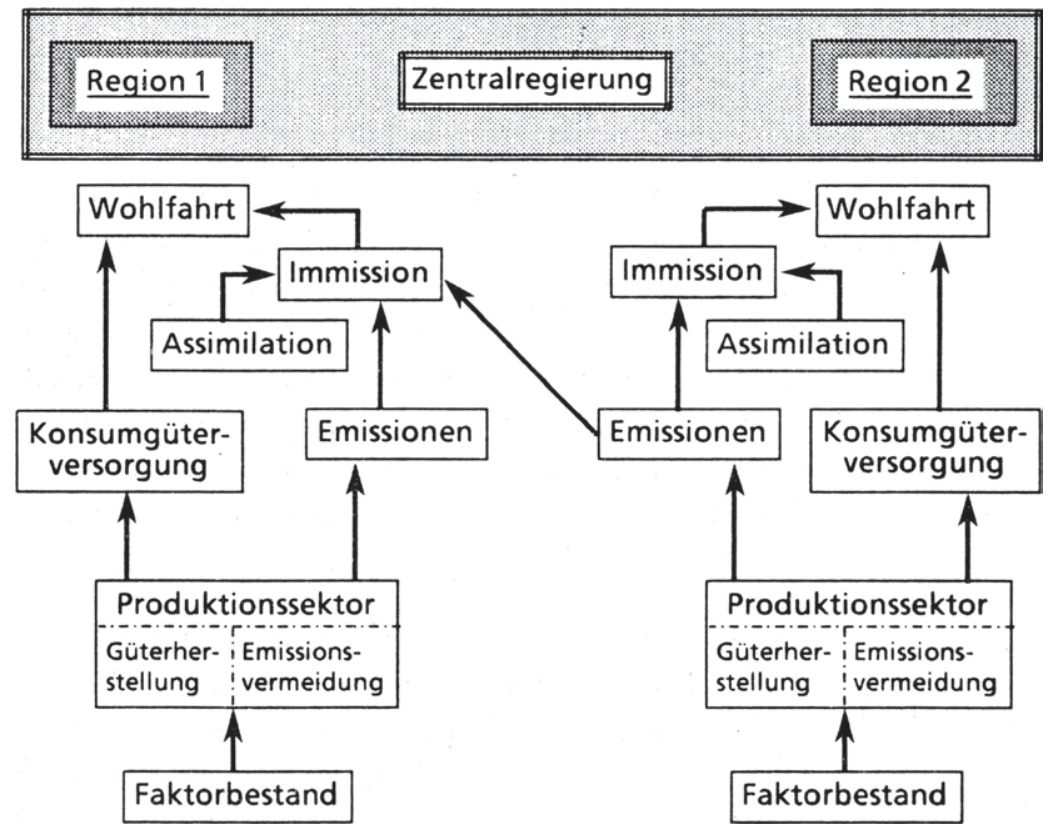

Schaubild 4.1: Die Struktur der Ökonomie

und die ökologischen Stationaritätsbedingungen sind

(i)

$$
S_{1}=\frac{1}{a_{1}}\left|E_{1}+Y_{2} E_{2}\right|
$$

(ii)

$$
S_{2}=\frac{1-\mathrm{Y}_{2}}{\mathrm{a}_{2}} E_{2}
$$

$\mathrm{Da}$ in diesem Kapitel die Faktoren und Güter annahmegemäß immobil sind, gilt zum einen die Faktorrestriktion

$$
\mathbf{R}_{i}-R_{i} \geq 0
$$

und zum anderen ist die konsumierte Menge an Gütern in Region $i$ gleich der produzierten Menge in Region $i$ und es gilt

$$
C_{i}=X_{i}
$$




\section{AUTONOME REGIONALBEHÖRDEN}

Hier wird davon ausgegangen, daß die Regionen vollkommen eigenständig sind. Die Planer jeder Region verfahren so, als ob sie sich innerhalb eines abgeschlossenen Systems befinden würden. Allerdings paßt sich die emissionsimportierende Region jeweils an die aus der anderen Region kommende Menge an Schadstoffen an (Cournot-Annahme). Es wird gezeigt, daß Region 1, d. h. die durch Emissionen der Region 2 mitverschmutzte Region, ihr Wohlfahrtskalkül nach der Umweltpolitik der anderen Region ausrichten muß. Wird in Region 2 eine relativ unwirksame, bzw. keine Umweltpolitik betrieben, sind die Emissionen in Region 2 hoch und damit auch der Anteil, der nach Region 1 gelangt. Für eine intertemporal wohlfahrtsoptimale Politik muß die Regionalregierung der Region 1 eine hohe Steuer setzen, so daß die Emissionen in Region 1 niedrig sind. Dies bedeutet aber, daß auch die Güterproduktion in Region 1 relativ niedrig ist, während die Verschmutzung, trotz der niedrigen Emissionen, hoch ist. Region 1 erleidet also durch die "laissez-faire" Politik in Region 2 Wohlfahrtsverluste durch eine relativ niedrige Güterversorgung und eine relativ schlechte Umweltqualität. Geht man von dem anderen Extrem aus, daß in Region 2 die Emissionen Null sind, d. h. dort wird eine sehr stringente Umweltpolitik betrieben, ist in Region 1 eine bessere Umweltqualität, höhere Emissionen und damit eine höhere Güterproduktion möglich als mit Emissionen aus Region 2, so daß in Region 1 Wohlfahrtsgewinne durch die Umweltpolitik der Region 2 zu verzeichnen sind.

\subsection{DiE OPTIMIERUNGSPROBLEME UND DIE OPTIMALBEDINGUNGEN}

Die Umweltbehörden der Region 2 sieht sich folgendem intertemporalen Optimierungsproblem gegenüber ${ }^{3)}$ :

3) Alle Variablen sind weiterhin Funktionen der Zeit; diese Abhängigkeit wird im folgenden aus schreibtechnischen Gründen nicht explizit ausgewiesen, sofern sich keine Mißdeutungen ergeben. 
(4.5.2) $\quad \operatorname{Max} W_{2}=\int_{0}^{\infty} u^{2}\left[X_{2}(t), S_{2}(t)\right] e^{-\delta t} d t$

u.d.B. (3.1) $\quad f^{\rho}\left(R_{2}, E_{2}\right)-X_{2} \geq 0$

(3.2) $a_{2} R_{2}-E_{2} \geq 0$

(4.3) $\quad \mathbf{R}_{2}-R_{2} \geq 0$

(4.1.ii) $\frac{d S_{2}}{d t}=\left(1-\mathrm{Y}_{2}\right) E_{2}-a_{2} S_{2}$

$X_{2}, R_{2}, E_{2} \geq 0 \forall t \in[0, \infty)$

$S_{2}(0)=S_{2}{ }^{0}$ gegeben

$\delta$ gegeben und positiv

Für Region 1 ergibt sich das analoge Problem:

(4.5.1) $\quad \operatorname{Max} W_{1}=\int_{0}^{\infty} u^{1}\left[X_{1}(t), S_{1}(t)\right] e^{-\delta t} d t$

$$
\begin{aligned}
& \text { u.d.B. (3.1) } \quad f^{\mathrm{l}}\left(R_{1}, E_{1}\right)-X_{1} \geq 0 \\
& \text { (3.2) } \quad a_{1} R_{1}-E_{1} \geq 0 \\
& \text { (4.3) } \quad R_{1}-R_{1} \geq 0 \\
& (4.1 . \mathrm{i}) \quad \frac{d S_{1}}{d t}=E_{1}+Y_{2} E_{2}-a_{1} S_{1} \\
& X_{1}, R_{1}, E_{1} \geq 0 \forall t \in[0, \infty) \\
& S_{1}(0)=S_{1}{ }^{0} \text { gegeben } \\
& \delta \text { gegeben und positiv } \\
& E_{2}=E_{2}{ }^{\infty} \text { gegeben. }
\end{aligned}
$$

Die so formulierten Probleme definieren ein nichtkooperatives NichtNullsummen-Differentialspiel, das jedoch auf zwei Kontrollprobleme zurückgeführt wird, indem der Wert der Emissionen aus Region 2 für Region 1 auf seinen Steady-State-Wert festgelegt wird. Das sich ergebende 
Gleichgewicht ist ein open-loop Nash-Gleichgewicht, in dem alle Werte stationär sind [vgl. Kapitel 3, Abschnitt 1.5.1].

Der Unterschied zu dem in Kapitel 3 untersuchten Grundmodell besteht darin, daß Region 2 einen Teil ihrer Emissionen nach Region 1 exportiert, ohne daß Region 1 Gegenmaßnahmen ergreifen kann. Die Regionalregierung in Region 1 betrachtet die Emissionen aus Region 2 als exogenen Faktor, auf den sie keinen Einfluß hat.

Die Lagrange-Funktionen in laufenden Werten sind $\left(\gamma_{1}=0, \gamma_{2}>0\right.$, $i=1,2)$ :

$$
\begin{aligned}
L^{i}=u^{i}\left(X_{i}, S_{i}\right)+ & p_{i}\left[\left(1-Y_{i}\right) E_{i}+\gamma_{j} E_{j}-\alpha_{i} S_{i}\right]+\lambda^{i}{ }_{1}\left[\dot{f}\left(R_{i}, E_{i}\right)-X_{i}\right]+ \\
& \lambda_{2}^{i}\left[a_{i} R_{i}-E_{i}\right]+\lambda_{3}^{i}\left[R_{i}-R_{i}\right]+\lambda^{i}{ }_{4} R_{i}+\lambda^{i}{ }_{5} E_{i}+\lambda^{i}{ }_{6} X_{i}
\end{aligned}
$$

woraus sich folgende Bedingungen ergeben:

(i)

$$
L_{X}^{i}=u_{X}^{i}-\lambda_{1}^{i}+\lambda_{6}^{i}=0
$$$$
\boldsymbol{X}_{i} \geq 0
$$

$$
L_{R}^{i}=\lambda^{i}{ }_{1} \dot{f}_{R}+\lambda^{i}{ }_{2} a_{i}-\lambda^{i}{ }_{3}+\lambda^{i}{ }_{4}=0
$$$$
R_{i} \geq 0
$$

$$
L_{E}^{1}=p_{1}+\lambda^{1}{ }_{1} f_{E}{ }_{E}-\lambda^{1}{ }_{2}+\lambda^{1}{ }_{5}=0
$$$$
E_{1} \geq 0
$$

$$
L_{E}^{2}=\left(1-\gamma_{2}\right) p_{2}+\lambda^{2}{ }_{1} f_{E}{ }_{E}-\lambda^{2}{ }_{2}+\lambda^{2}{ }_{5}=0
$$$$
E_{2} \geq 0
$$

$$
L_{p}^{1}=\frac{d S_{1}}{d t}=E_{1}+\mathrm{Y}_{2} E_{2}-a_{1} S_{1}
$$

(iv.2)

$$
L_{p}^{2}=\frac{d S_{2}}{d t}=\left(1-Y_{2}\right) E_{2}-a_{2} S_{2}
$$

$$
L_{S}^{i}=-\frac{d p_{i}}{d t}+\delta p_{i}=u_{S}^{i}-a_{i} p_{i}
$$

$$
\lambda_{1}^{i}\left[\dot{f}^{(}\left(R_{i}, E_{i}\right)-X_{i}\right]=0
$$

$$
\lambda_{2}^{i}\left[a_{i} R_{i}-E_{i}\right]=0
$$$$
\lambda_{2}^{i} \geq 0
$$$$
\lambda^{i}{ }_{3} \geq 0
$$

$$
\lambda^{i}{ }_{3}\left[\mathbf{R}_{i}-R_{i}\right]=0
$$$$
\lambda_{4}^{i} \geq 0
$$ 
$(\mathbf{x})$

(xi)

$$
\lambda^{i}{ }_{5} E_{i}=0
$$$$
\lambda_{6}^{i} X_{i}=0
$$

$\lambda_{5}^{i} \geq 0$

$\lambda_{6}^{i} \geq 0$

Aus den Wohlfahrtsfunktionen folgt wiederum, daß positiver Güterkonsum vorliegen soll: $X_{i}>0$, daraus folgt dann $R_{i}>0$ und damit $\lambda_{4}^{i}=\lambda_{6}^{i}$ $=\mathbf{0}$. D. h.

$$
u_{X}^{i}=\lambda_{1}^{i}>0
$$

sowie

$$
\lambda_{1}^{i} i_{\mathrm{R}}+\lambda_{2}^{i} a_{i}=\lambda_{3}^{i}>0,
$$

was Vollbeschäftigung impliziert $\left(R_{i}=\mathbf{R}_{i}\right)$.

Die Besteuerungsfunktionen sind wieder

$$
\tau_{i}=u_{X}^{i} f_{E}
$$

aus denen sich die Funktionen

$$
E_{i}=E^{i}\left(\tau_{i}\right), \quad E_{\tau}^{i}<0
$$

ableiten lassen, die die gleichen Eigenschaften wie (3.26) haben.

Betrachten wir jetzt die Zuordnung zwischen Steuer und Schattenpreis; für Region 1 erhalten wir

(4.10) $\tau_{1}=\left\{\begin{array}{lll}\tau_{1}{ }^{\prime} & \text { für } p_{1} \geq p_{1}^{\prime}, & p_{1}{ }^{\prime} \equiv-\tau_{1}{ }^{\prime} \\ -p_{1} & \text { für } p_{1} \in\left(p_{1}^{\prime}, p_{1}{ }^{\prime \prime}\right) & p_{1}{ }^{\prime \prime} \equiv-\tau_{1}{ }^{\prime \prime} \\ \tau_{1}{ }^{\prime} & \text { für } p_{1} \leq p_{1}{ }^{\prime \prime}\end{array}\right.$

d. h. für Region 1 entspricht die Steuer dem Zukunftsschaden $\left(-p_{1}\right)$, den eine Emissionseinheit verursacht. Für Region 2 ist die optimale Steuer dagegen folgendermaßen definiert: 
(4.11) $\quad \tau_{2}=\left\{\begin{array}{lll}\tau_{2}{ }^{\prime} & \text { für } p_{2} \geq p_{2}^{\prime}, \quad p_{2}^{\prime} \equiv \frac{-\tau_{2}{ }^{\prime}}{1-\gamma_{2}} \\ -\left(1-\gamma_{2}\right) p_{2} & \text { für } p_{2} \in\left(p_{2}^{\prime}, p_{2}{ }^{\prime \prime}\right), \quad p_{2}{ }^{\prime \prime} \equiv \frac{-\tau_{2}{ }^{\prime \prime}}{1-\gamma_{2}} \\ \tau_{2}{ }^{\prime \prime} & \text { für } p_{2} \leq p_{2}{ }^{\prime \prime} & \end{array}\right.$

Da nur das $\left(1-\gamma_{2}\right)$-fache einer Emissionseinheit in Region 2 verbleibt, ist die Steuer nur das $\left(1-\gamma_{2}\right)$-fache der intertemporalen Wohlfahrtsänderung durch eine zusätzliche Emissionseinheit [ für $E_{2} \in\left(0, E_{2}^{\max }\right)$ ].

Für bis auf die Diffusionsbeziehungen identische Regionen sind die Beziehungen zwischen Steuer, Schattenpreis und Emissionen in Schaubild 4.2 dargestellt. Es ist ersichtlich, daß für den gleichen Schattenpreis $\mathbf{p}_{i}$ in beiden Regionen, in Region 1 weniger Emissionen produziert werden dürfen während die Steuer höher ist als in Region 2.

Wir gehen jetzt davon aus, daß eine innere Lösung existiert, so daß gilt $E_{i} \in\left(0, E_{i}^{\text {max }}\right)$. Wir setzen (4.10) bzw. (4.11) in (4.9) ein und dies wiederum in (4.7.iv.1) bzw. (4.7.iv.2). Dann erhalten wir folgende Differentialgleichungssysteme

$$
\frac{d S_{1}}{d t}=E_{1}\left[-p_{1}\right]+\mathrm{Y}_{2} E_{2}-a_{1} S_{1}
$$

$$
\frac{d p_{1}}{d t}=\left(\delta+a_{1}\right) p_{1}-u_{S}^{1}
$$

(i)

$$
\frac{d S_{2}}{d t}=\left(1-\mathrm{Y}_{2}\right) E_{2}\left[-\left(1-\mathrm{Y}_{2}\right) p_{2}\right]-a_{2} S_{2}
$$

(ii)

$$
\frac{d p_{2}}{d t}=\left(\delta+a_{2}\right) p_{2}-u_{S}^{2}
$$

Das Differentialgleichungssystem (4.13) kann man für eine gegebene Anfangsverschmutzung $S_{2}{ }^{0}$ und ein geeignet gewähltes $p_{2}(0)$ lösen, da die Argumente der Funktionen $d S_{2} / d t$ und $d p_{2} / d t$ stetig sind und zumindest in- 


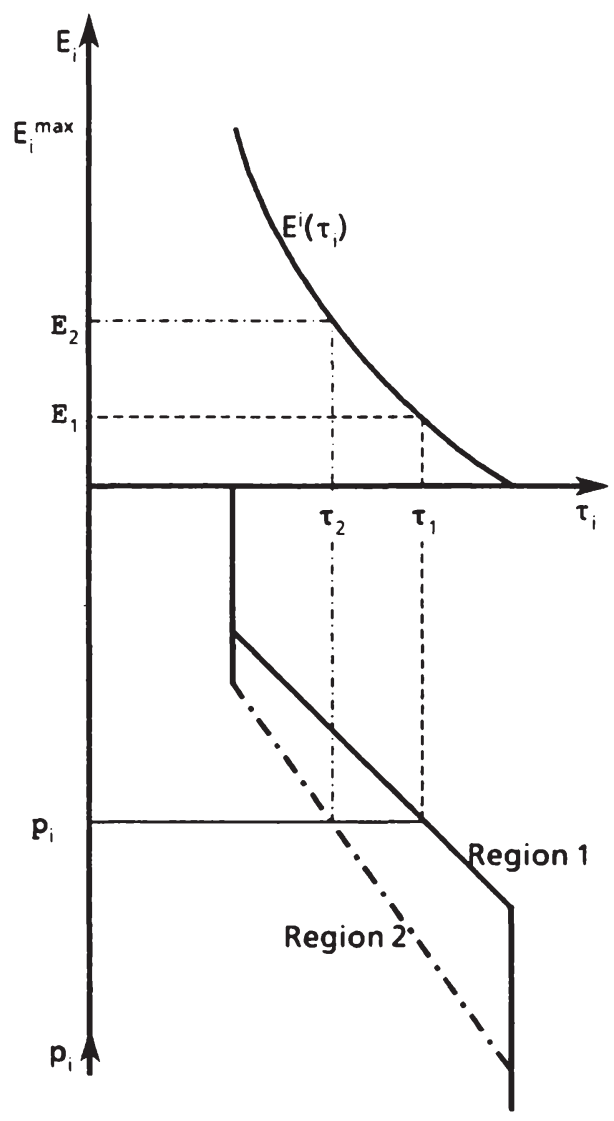

Schaubild 4.2: Die Beziehungen zwischen Steuern, Emissionen und Schattenpreisen

tervallweise stetige erste Ableitungen haben ${ }^{4)}$. Das System für Region 2 weist ein eindeutiges stationäres Gleichgewicht $\left(S_{2}{ }^{\infty}, p_{2}{ }^{\infty}\right)$ auf, das einen Sattelpunkt ${ }^{5}$ darstellt. Man erhält dann einen eindeutigen Wert $E_{2}{ }^{\infty}$, der in (4.12.i) eingesetzt wird, so daß auch das Differentialgleichungssystem für Region 1 für einen gegebenen Anfangsimmissionsbestand $S_{1}{ }^{0}$ und ein

4) Vgl. Hadley, Kemp (1971, 370, Theorem A III - 1).

5) Vgl. Fußnote 33, Kapitel 3. 
geeignet gewähltes $p_{1}(0)$ ein eindeutiges stationäres Gleichgewicht $\left(S_{1}{ }^{\infty}, p_{1}{ }^{\infty}\right)$ besitzt. Jedoch ist diese Lösung nicht unabhängig von der Lösung in Region 2, so daß die Bewegungseigenschaften des Differentialgleichungssystems für Region 1 mit von den Bewegungseigenschaften des Differentialgleichungssystems für Region 2 abhängen.

\subsection{DIE ZEITLICHE ENTWICKLUNG IN REGION 2}

In Schaubild 4.3 wird mit der Phasendiagramm-Methode der Optimalpunkt der Region 2 bestimmt:

Im I. Quadranten ist die stationäre Ökozustand-Funktion der Region 2 eingezeichnet:

$$
S_{2}=\frac{1-Y_{2}}{a_{2}} E_{2}
$$

d. h. bezüglich $S_{2}$ verläuft diese Gerade flacher als ohne Emissionsexport. Durch diesen Export wird praktisch die Assimilationsfähigkeit in Region 2 erhöht. Im II. Quadranten ist die Steuerfunktion $E^{2}\left(\tau_{2}\right)$ eingezeichnet und im III. Quadranten wird die Beziehung zwischen der optimalen Steuer $\tau_{2}$ und dem Schattenpreis $p_{2}$ dargestellt. Auch hier gibt es einen Unterschied zum Grundmodell: Für eine autonome Regionalbehörde ist es optimal, daß durch die Steuer nicht der gesamte durch die Emissionen hervorgerufene Zukunftsschaden ausgeglichen wird, sondern nur der in Region 2 verbleibende Teil der Emissionen wird berücksichtigt. Deshalb verläuft diese Gerade steiler als ohne Emissionsexport, d. h. die Steuer ist niedriger als der Schattenpreis. Im IV. Quadranten schließlich ist einmal die $d p_{2} / d t=0$ Kurve

$$
p_{2}=\frac{u_{S}^{2}}{\delta+a_{2}}
$$

eingezeichnet. Oberhalb dieser Kurve nimmt der Schattenpreis zu, während er unterhalb abnimmt. Zum anderen ist die abgeleitete $d S_{2} / d t=0$ Kurve eingezeichnet: 


$$
S_{2}=\frac{1-Y_{2}}{a_{2}} E^{2}\left[-\left(1-Y_{2}\right) p_{2}\right]
$$

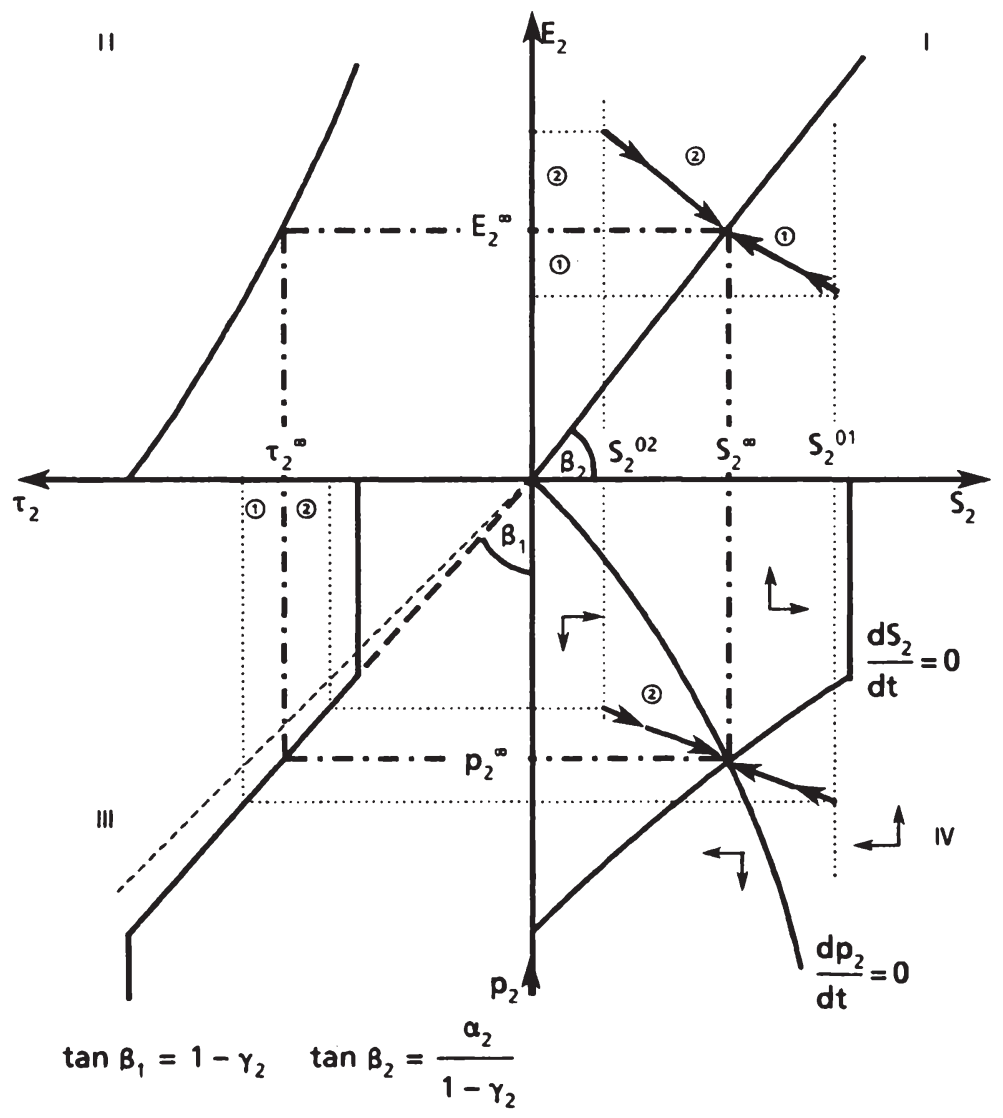

Schaubild 4.3: Die zeitliche Entwicklung von Region 2

Diese Kurve hat eine positive Steigung und links der Kurve ist die Bewegungsrichtung der Immissionen positiv, während sie rechts negativ ist. Man erhält vier Sektoren, in denen die Bewegungseigenschaften des Sy- 
stems jeweils unterschiedlich sind. Zur weiteren Diskussion vgl. das Grundmodell Kapitel 3, Abschnitt 2.1, die sich für die Anpassungen in Region 2 vollkommen analog anwenden läßt unter Berücksichtigung oben genannter Unterschiede.

\subsection{DiE ZEITLICHE ENTWICKLUNG IN REGION 1}

Für Region 1 gelten im Prinzip die gleichen Aussagen, jedoch ergeben sich durch den Emissionsimport einige Unterschiede: Die $d p_{1} / d t=0$ Kurve ist genauso abzuleiten wie die $d p_{2} / d t=0 \mathrm{Kurve}$

$$
p_{1}=\frac{u_{S}^{1}}{\delta+a_{1}}
$$

Für die $d S_{1} / d t=0$ Kurve kann kein bestimmter Verlauf mehr angegeben werden, da der stationäre Ökozustand mit von den Emissionen der Region 2 abhängt:

$$
S_{1}=\frac{1}{a_{1}}\left|E^{1}\left[-p_{1}\right]+Y_{2} E_{2}\right|
$$

In Schaubild 4.4 sind drei verschiedene Verläufe von $d S_{1} / d t=0$ eingezeichnet, je nachdem wie groß $E_{2}$ ist:

(I) $E_{2}=0$

Für die $d S_{1} / d t=0$ Kurve ergibt sich

$$
S_{1}=\frac{1}{a_{1}} E^{1}\left[-p_{1}\right] \quad \text { mit } \quad \frac{d S_{1}}{d p_{1}}=-\frac{1}{a_{1}} E_{\tau}^{1}>0
$$

d. h. der stationäre Ökozustand ist nur von der Verschmutzung und der Emission in Region 1 abhängig. Hier ist für Region 1 der Fall des Grundmodells in Kapitel 3 gegeben. 
(II) $E_{2}=E_{2}^{*} \in\left(0, E_{2}^{\max }\right)$

Für die $d S_{1} / d t=0$ Kurve erhält man

$$
S_{1}=\frac{1}{a_{1}}\left[E^{1}\left[-p_{1}\right]+\gamma_{2} E_{2}^{*}\right] \quad \text { mit } \quad \frac{\partial S_{1}}{\partial p_{1}}=-\frac{1}{a_{1}} E_{\tau}^{1}>0
$$

d. h. der stationäre Ökozustand der Region 1 ist abhängig von den Emissionen der Region 2. Da $\partial S_{1} / \partial E_{2}{ }^{*}=\gamma_{2} / \alpha_{1}>0$ ist, ist $S_{1}$ umso größer, je größer $E_{2}{ }^{*}$ ist.

(III) $E_{2}=E_{2}{ }^{\max }$

Dann folgt

$$
S_{1}=\frac{1}{a_{1}}\left|E^{1}\left[-p_{1}\right]+Y_{2} E_{2}^{\max }\right| \quad \text { mit } \quad \frac{\partial S_{1}}{\partial p_{1}}=-\frac{1}{a_{1}} E_{\imath}^{1}>0
$$

Zusammenfassend läßt sich sagen, daß je größer $E_{2}$ ist, desto weiter nach außen verschiebt sich die $d S_{1} / d t=0 \mathrm{Kurve}$. Diese Verschiebung erfolgt parallel, da im $\left(p_{1}, S_{1}\right)$-Diagramm die $d S_{1} / d t=0$ Kurven jeweils die gleiche Steigung haben. Ist z. B. $E_{2}=E_{2}{ }^{\max }$, d. h. in Region 2 wird die Steuer niedrig gesetzt bzw. es wird keine Umweltpolitik betrieben, dann muß in Region 1 eine stringente Umweltpolitik betrieben werden, um ins Optimum zu gelangen. Jedoch sind dann die Immissionen hoch, während der Güteroutput aufgrund niedriger Emissionen, d. h. einer hohen Steuer, relativ klein ist. Ist dagegen $E_{2}=0, \mathrm{~d}$. h. in Region 2 wird eine sehr strenge Umweltpolitik betrieben, kann in Region 1 ein Wohlfahrtsoptimum verwirklicht werden, in dem die Immissionen relativ niedrig sind, während die Emissionen hoch sind und damit ein relativ großer Güteroutput erstellt werden kann.

Es ist ersichtlich, daß für $E_{1}=0$ die $d S_{1} / d t=0$ Kurve nur dann die $p_{1}$-Achse schneidet, wenn auch $E_{2}=0$ ist. Ansonsten ist immer noch eine Verschmutzung der Region 1 vorhanden, wenn $E_{2}>0$ ist, auch wenn $E_{1}=0$ ist, d. h. $\tau_{1}>\tau_{1}{ }^{\prime \prime}$ ist.

Der optimale Anfangswert des Immissionsschattenpreises $p_{1}(0)$ variiert c. p. mit der Anfangsverschmutzung in Region 1 sowie den "importierten" Emissionen aus Region 2: 


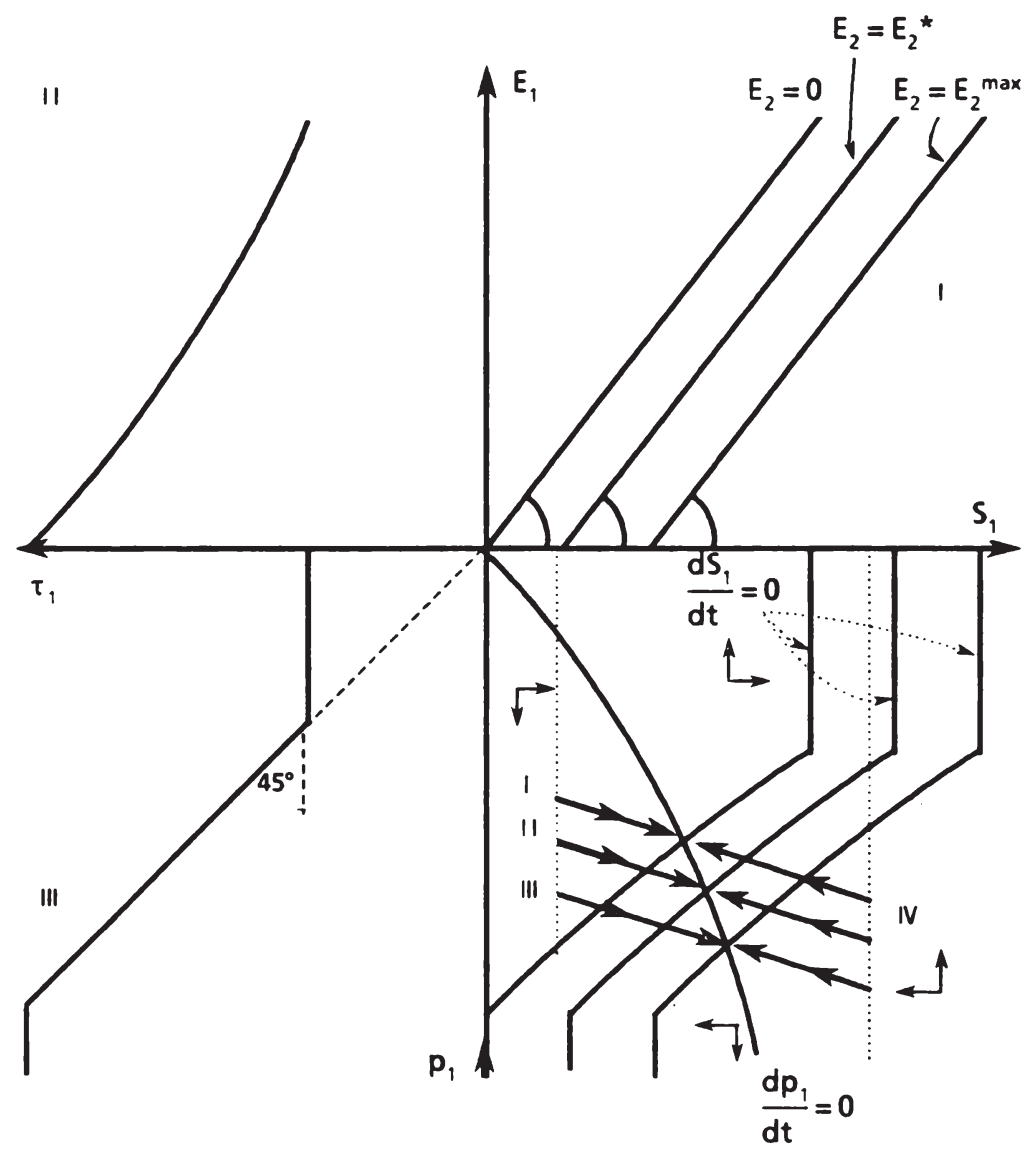

Schaubild 4.4: Die zeitliche Entwicklung von Region 1

a) bezüglich der Anfangsverschmutzung muß der Schattenpreis $p_{1}(0)$ c.p. umso niedriger (höher) sein, je größer (kleiner) die Anfangsverschmutzung ist,

b) die Emissionen der Region 2 hängen vom Immissionsschattenpreis $p_{2}$ und damit der Steuer $\tau_{2}$ der Region 2 ab. Je niedriger $\tau_{2}$ ist, d. h. je mehr in Region 2 emittiert wird, desto mehr Emissi- 
onen gelangen nach Region 1 , so daß $p_{1}(0)$ niedrig, d. h. die Steuer $\tau_{1}$ hoch, gesetzt werden muß. Ist andererseits $\tau_{2}$ niedrig, es wird in Region 2 wenig emittiert, kann $\tau_{1}(0)$ niedrig angesetzt werden. Dies bedeutet aber, daß die Umweltpolitik der Region 1 von der Umweltpolitik der Region 2 mitabhängig ist.

Aus den Gleichungen (4.16), (4.17) und (4.18) erhält man Differentialgleichungen

$$
\begin{aligned}
\frac{d S_{1}^{*}}{d t}=\frac{\mathrm{V}_{2}\left(\delta+a_{1}\right)}{a_{1}\left(\delta+a_{1}\right)+E_{\mathrm{v}}^{1} u_{S S}^{1}} \frac{d E_{2}}{d t} & >0 \text { für } S_{2}{ }^{0}>S_{2}{ }^{\infty} \\
\frac{d p_{1}^{*}}{d t}=\frac{u_{S S}^{1}}{\left(\delta+a_{1}\right)} \frac{d S_{1}^{*}}{d t} & <\text { für } S_{2}{ }^{0}=S_{2}{ }^{\infty} \\
& <0 \text { für } S_{2}{ }^{0}<S_{2}{ }^{\infty} \\
& <0 \text { für } S_{2}{ }^{0}>S_{2}{ }^{\infty} \\
& =0 \text { für } S_{2}{ }^{0}=S_{2}{ }^{\infty} \\
& >0 \text { für } S_{2}{ }^{0}<S_{2}{ }^{\infty}
\end{aligned}
$$

wobei $\left(S_{1}{ }^{*}, p_{1}{ }^{*}\right)$ "Quasi-Steady-States" symbolisieren, d. h. "SteadyStates", die bei jeweils festen $E_{2}$ erreicht würden, wenn sich Region 1 auf der jeweiligen "Optimaltrajektorie" dorthin bewegt. Da sich jedoch $E_{2}$ ändern kann, stimmen diese Quasi-Steady-States nur dann mit dem stationären Gleichgewicht $\left(S_{1}{ }^{\infty}, p_{1}{ }^{\infty}\right)$ überein, wenn die Ausgangsverschmutzung in Region 2 mit der stationären Verschmutzung übereinstimmt $\left(S_{2}{ }^{0}\right.$ $\left.=S_{2}{ }^{\infty}\right)$. Geht man von $S_{2}{ }^{0}>S_{2}{ }^{\infty}$ im Zeitpunkt 0 aus, so ergibt sich aus (4.19), daß der Quasi-Steady-State Wert der Immissionen in Region 1 im Zeitpunkt 0 kleiner ist als der Steady-State Wert $S_{1}{ }^{\infty}$, d. h. $S_{1}$ steigt im Zeitablauf so lange bis $d E_{2} / d t=0$ ist. Aus (4.20) erhält man die zeitliche Entwicklung des Quasi-Steady-State Wertes des Immissionsschattenpreis $p_{1}{ }^{*}$. Dieser sinkt im Zeitablauf so lange, bis $S_{2}{ }^{\infty}$ erreicht ist, bis sich Region 2 im Steady-State befindet. Dieser zeitliche Ablauf wird in Schaubild 4.4 dargestellt, wobei die Pfeile die Bewegungsrichtung angeben. Je mehr $E_{2}$ also steigt, desto weiter verschiebt sich die $d S_{1} / d t=0$ Kurve parallel nach außen, und damit auch ihr Schnittpunkt mit der $d p_{1} / d t=0$ Kurve, so daß die Optimaltrajektorien sich ebenfalls nach unten verschieben. Gilt $S_{2}{ }^{0}<S_{2}{ }^{\infty}$ ergeben sich die analogen Anpassungen der Quasi-SteadyStates in Region 1. 


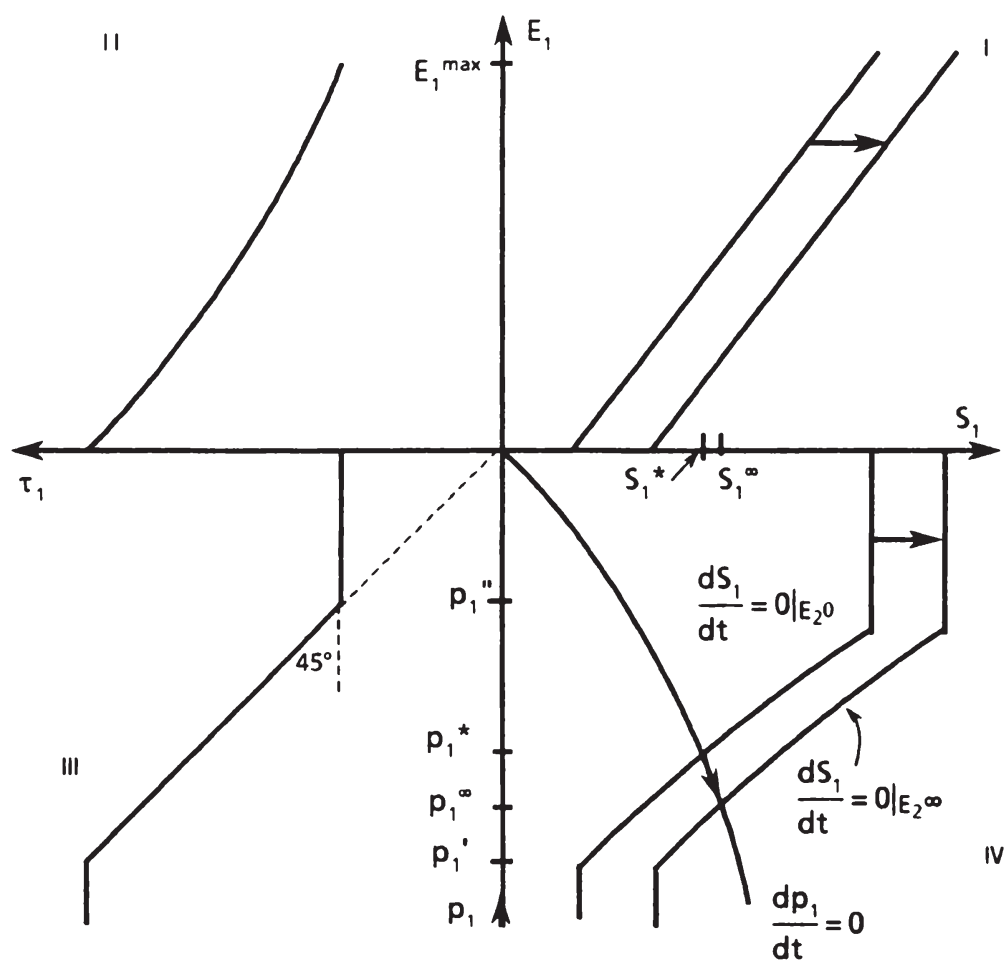

Schaubild 4.5: Die zeitliche Entwicklung des Quasi-Steady-State Wertes in Region 1

\subsection{DAS GESAMT-STEADY-STATE DER ÖKONOMIE}

\subsubsection{Die Ableitung des Gesamt-Steady-States}

Jetzt definieren wir ein Gesamt-Steady-State der Ökonomie als einen Zustand, bei dem alle Werte stationär sind. Dann kann man für Region 1 "Steady-State-Reaktionsfunktionen" ableiten: Totale Differentiation der Steady-State-Funktionen und der Steuerfunktion nach $E_{2}$ ergibt 
$(4.21)\left[\begin{array}{cccc}1 & -\alpha_{1} & 0 & 0 \\ 0 & -u_{S S}^{1} & \delta+\alpha_{1} & 0 \\ 0 & 0 & 1 & 1 \\ 1 & 0 & 0 & -E_{\tau}^{1}\end{array}\right]\left[\begin{array}{c}\frac{d E_{1}^{*}}{d E_{2}} \\ \frac{d S_{1}^{*}}{d E_{2}} \\ \frac{d p_{1}^{*}}{d E_{2}} \\ \frac{d \tau_{1}^{*}}{d E_{2}}\end{array}\right]=\left[\begin{array}{c}-\gamma_{2} \\ 0 \\ 0 \\ 0\end{array}\right]$

Die Systemdeterminante ist

$$
\Delta_{1}=u_{S S}^{1} E_{\tau}^{1}+\alpha_{1}\left(\delta+\alpha_{1}\right)>0
$$

Für die Änderungen der Steady-State-Werte in Abhängigkeit der importierten Emissionen gilt ${ }^{6)}$

$$
\begin{aligned}
& \frac{d E_{1}^{*}}{d E_{2}}=\frac{-\mathrm{Y}_{2} u_{S S}^{1} E_{\mathrm{l}}^{1}}{\Delta_{1}}<0 \\
& \frac{d S_{1}^{*}}{d E_{2}}=\frac{\mathrm{Y}_{2}\left(\delta+\mathrm{a}_{1}\right)}{\Delta_{1}}>0 \\
& \frac{d p_{1}^{*}}{d E_{2}}=\frac{\mathrm{Y}_{2} u_{S S}^{1}}{\Delta_{1}}<0 \\
& \frac{d \tau_{1}^{*}}{d E_{2}}=-\frac{\mathrm{Y}_{2} u_{S S}^{1}}{\Delta_{1}}>0
\end{aligned}
$$

Im weiteren betrachten wir die Funktion

$$
E_{1}^{*}=\varepsilon^{1}\left(E_{2}\right), \quad \varepsilon_{E}^{1} \in\left(-\gamma_{2}, 0\right)
$$

6) Zu dieser Methode vgl. Bernstein (1977). 
Ein Gesamtgleichgewicht ist dann definiert als

$$
E_{1}^{\infty}=\varepsilon^{1}\left(E_{2}^{\infty}\right)
$$

Dieses Gesamt-Steady-State wird in Schaubild 4.6 graphisch abgeleitet.

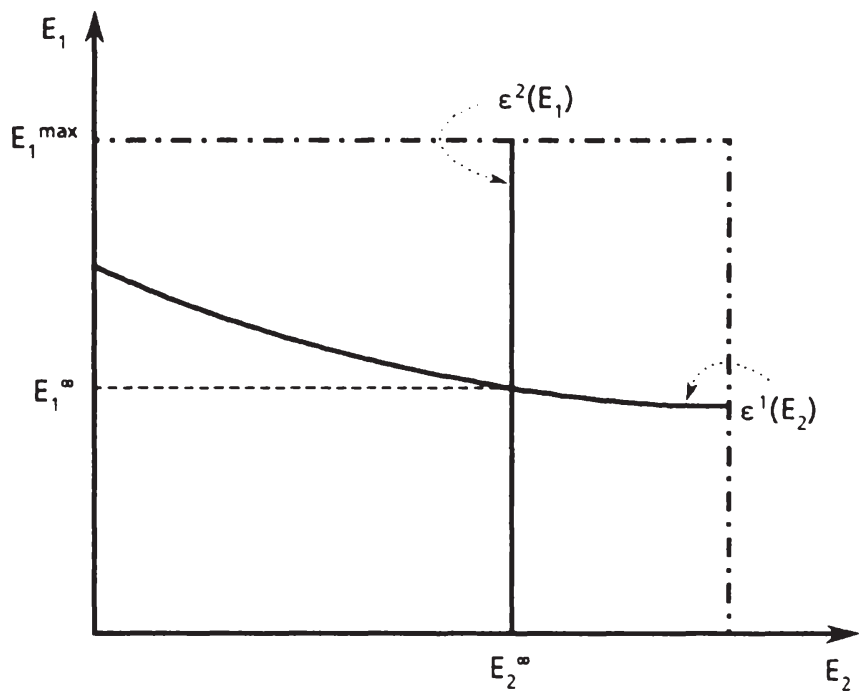

Schaubild 4.6: Die Steady-State-Reaktionsfunktion der Region 1

Die "Steady-State-Reaktionsfunktion" der Region $2 \varepsilon^{2}\left(E_{1}\right)$ ist als Vertikale in Schaubild 4.6 eingezeichnet, da die optimale Emissionsmenge in Region 2 vollkommen unabhängig von der Emissionsmenge in Region 1 ist. Der Schnittpunkt von $\varepsilon^{1}\left(E_{2}\right)$ mit $\varepsilon^{2}\left(E_{1}\right)$ ergibt dann das Gesamt-SteadyState der Ökonomie.

\subsubsection{Auswirkungen von Parameteränderungen auf das Steady-State}

Die Größe der Steady-State-Werte der Emissionen hängt ab von dem Diffusionskoeffizienten $\gamma_{2}$, der Ressourcenausstattung der Regionen $\mathbf{R}_{i}$, der 
sozialen Zeitpräferenzrate $\delta$ sowie den Eigenschaften des Ökosystems, hier ausgedrückt durch $\alpha_{i}$, dem Assimilationskoeffizienten. Welche Auswirkungen unterschiedliche Größen dieser Parameter haben wird nun im folgenden untersucht. Die Effekte der Änderung dieser Parameter auf die Systemvariablen $E_{i}, S_{i}, \tau_{i}, p_{i}, X_{i}$ sind in folgender Tabelle zusammengefaßt ${ }^{7}$.

\begin{tabular}{|c|c|c|c|c|c|c|c|c|c|c|}
\hline & $d E_{1}$ & $d S_{1}$ & $d p_{1}$ & $d \tau_{1}$ & $d X_{1}$ & $d E_{2}$ & $d S_{2}$ & $d p_{2}$ & $d \tau_{2}$ & $d X_{2}$ \\
\hline$d \delta$ & $?$ & $\oplus$ & $?$ & $?$ & $?$ & $\oplus$ & $\oplus$ & $\oplus$ & $\ominus$ & $\oplus$ \\
\hline$d \alpha_{1}$ & $\oplus$ & $?$ & $\oplus$ & $\Theta$ & $\oplus$ & $\bullet$ & $\bullet$ & $\bullet$ & $\bullet$ & $\bullet$ \\
\hline$d \mathbf{R}_{1}$ & $\oplus$ & $\oplus$ & $\Theta$ & $\oplus$ & $\oplus$ & $\bullet$ & $\bullet$ & $\bullet$ & $\bullet$ & $\bullet$ \\
\hline$d \alpha_{2}$ & $\Theta$ & $\oplus$ & $\Theta$ & $\oplus$ & $\Theta$ & $\oplus$ & $?$ & $\oplus$ & $\Theta$ & $\oplus$ \\
\hline$d \mathbf{R}_{2}$ & $\Theta$ & $\oplus$ & $\Theta$ & $\oplus$ & $\Theta$ & $\oplus$ & $\oplus$ & $\ominus$ & $\oplus$ & $\oplus$ \\
\hline$d \gamma_{2}$ & $\Theta$ & $\oplus$ & $\Theta$ & $\oplus$ & $\Theta$ & $\oplus$ & $?$ & $?$ & $\Theta$ & $\oplus$ \\
\hline
\end{tabular}

keine Auswirkungen

Tabelle 4.1: Auswirkungen von Parameteränderungen

\subsubsection{Die Auswirkungen der Höhe der sozialen Zeitpräferenzrate}

Die Höhe der sozialen Diskontrate hat auf das Steady-State der Region 2 die gleichen Auswirkungen wie in Kapitel 3 dargestellt. Für Region 1 jedoch gilt es zu untersuchen, ob die Auswirkungen des erhöhten Emissionsimports aus Region 2 die niedrigere Bewertung des Zukunftsschadens und damit der zukünftigen Wohlfahrt kompensiert oder nicht.

Es gilt

$$
\begin{aligned}
& \frac{d E_{1}}{d \delta} \geq 0 \Leftrightarrow \frac{d p_{1}}{d \delta} \geq 0 \Leftrightarrow \frac{d \tau_{1}}{d \delta}>0 \Leftrightarrow \frac{d X_{1}}{d \delta} \geq 0 \Leftrightarrow \\
& \left.\frac{d S_{1}}{d E_{2}}\right|_{d E_{1}=0} E_{\delta}^{2}-\left.\frac{d S_{1}}{d \delta}\right|_{d p_{1}=0} \geq_{0}
\end{aligned}
$$

(1)

(2)

7) Die Ableitung dieser Effekte erfolgt im Anhang. 
Ist der Effekt durch die erhöhten Emissionen aus Region 2 auf die Immissionen (bei konstanten Emissionen in Region 1) [Effekt (1)] größer (kleiner) als der Effekt auf die Immissionen durch die größere Gegenwartsvorliebe bei konstantem Zukunftsschaden [Effekt (2)], dann sinken (steigen) die Emissionen und der Konsum in Region 1, während der bewertete $\mathrm{Zu}$ kunftsschaden und die Steuer steigen (sinken). In Schaubild 4.7 ist der Fall dargestellt, daß Effekt (2) Effekt (1) überkompensiert.

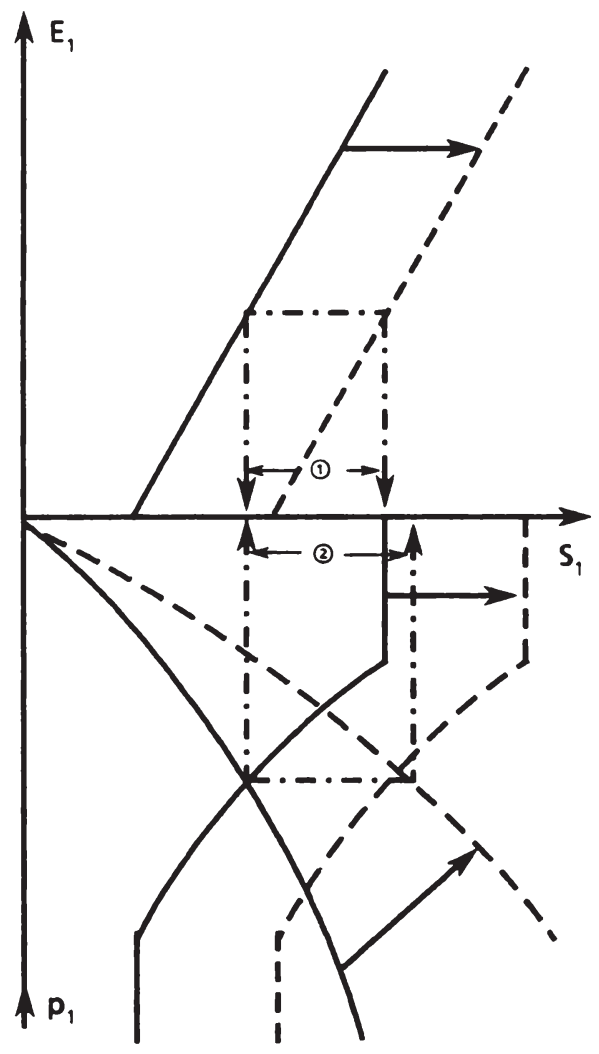

Schaubild 4.7: Die Effekte bei einer Änderung der

Gegenwartsvorliebe in Region 1 


\subsubsection{Die Auswirkungen der Höhe der Assimilationskoeffizienten}

Betrachten wir jetzt die Assimilationskoeffizienten: Hier bleiben die Aussagen, die im Exkurs in Kapitel 3, Abschnitt 2.2 gemacht wurden, für die jeweilige Region erhalten. Es treten auch hier wieder die beiden Effekte auf, der Emissions-Steuer-Effekt und der reine Emissionseffekt, die die Höhe der Steady-State Immissionen bestimmen. Für Region 1 kommen jedoch wieder die Effekte durch die Abhängigkeit von Region 2 durch den Emissionsimport hinzu. Es gilt: Je größer der Assimilationskoeffizient in Region 2, $\alpha_{2}$, ist, umso niedriger sind die Emissionen in Region 1 im Steady-State und damit auch der Konsum, jedoch umso höher sind die Immissionen und der Zukunftsschaden und damit auch die Steuer.

Zusammenfassend läßt sich sagen, daß, wenn in Region 2 eine hohe Assimilationsfähigkeit gegeben ist, Region 1 ihre Produktion stark einschränken muß, um eine durch die Nutzenfunktion definierte optimale Kombination Konsumgut - Immissionen zu erreichen.

\subsubsection{Die Auswirkungen der Höhe der Ressourcenausstattungen}

Es ist klar, daß die Ressourcenausstattung und die Eigenschaften des Ökosystems der Region 1 keine Auswirkungen auf das Verhalten der Region 2 haben. Umgekehrt gilt dies jedoch nicht. Für die Ressourcenausstattung gilt, daß je größer der jeweilige Faktorbestand ist, desto höher sind die Steady-State Emissionen und damit auch die Immissionen, die Steuer und der Konsum in der jeweiligen Region. Auch der Zukunftsschaden ist größer $\left(p_{i}^{\infty} \text { ist kleiner }\right)^{8}$. Die Größe des Faktorbestandes in Region 2 hat über die Emissionsdiffussion auch Auswirkungen auf Region 1: Je größer der Faktorbestand in Region 2 ist, desto geringer sind die Emissionen und der Konsum in Region 1, während die Immissionen, der Zukunftsschaden $\left(p_{1}{ }^{\infty}\right.$ sinkt) und die Steuer umso größer werden.

8) Diese Effekte sind unabhängig von der interregionalen Beziehung über die Diffusion in der Richtung, jedoch für Region 2 nicht im Ausmaß. Vgl. Anhang 3 und Anhang 4.I. 


\subsubsection{Die Auswirkungen der Höhe des Diffusionskoeffizienten}

Als letztes betrachten wir den Effekt, der durch den Emissionsexport der Region 2 hinzukommt. Es wird untersucht, welche Auswirkungen eine Erhöhung des Diffusionskoeffizienten auf die Variablen hat. Wie aus Tabelle 4.1 ersichtlich, ergeben sich eindeutige Auswirkungen auf Region 1. Da die Emissionen in Region 2 steigen und deshalb ein größerer Anteil in das Ökosystem der Region 1 gelangt, muß Region 1 Wohlfahrtsverluste hinnehmen: Die Steady-State-Immissionen steigen und der Steady-StateKonsum sinkt, beides hat negative Wohlfahrtseffekte für Region 1. In Schaubild 4.8 sind die Änderungen für Region 1 dargestellt. Die Pfeile geben die Änderungsrichtung an.

Für Region 2 erhalten wir eindeutige Effekte bezüglich der Emissionen $\left[d E_{2}{ }^{\infty} / d \gamma_{2}>0\right]$, der Steuer $\left[d \tau_{2}^{\infty} / d \gamma_{2}<0\right]$ und dem Konsum $\left[d X_{2}^{\infty} / d \gamma_{2}>0\right]$. Nicht eindeutig ist dagegen die Entwicklung der Immissionen und des $\mathrm{Zu}$ kunftsschadens. Es gilt

$$
\begin{aligned}
& \frac{d S_{2}}{d \mathrm{Y}_{2}} \geq 0 \Leftrightarrow \frac{d p_{2}}{d \mathrm{Y}_{2}}>0 \Leftrightarrow \\
& E_{\tau}^{2} \frac{\tau_{2}}{E_{2}}>-1
\end{aligned}
$$

Ist die Steuerelastizität der Emissionen in Region 2 größer (kleiner) als Eins, d. h. die Emissionen sinken bei einer Steuererhöhung überproportional (unterproportional), dann nehmen bzw. nimmt die Immissionen bzw. der Zukunftsschaden bei einer exogenen Erhöhung des Diffusionsparameters zu (ab). Die Effekte einer Erhöhung des Diffusionskoeffizienten auf Region 2 sind in Schaubild 4.9 zusammengefaßt. Die Pfeile in Schaubild 4.9 geben die Änderungsrichtung bei einer Erhöhung des Diffusionskoeffizienten an. Betrachten wir zuerst den Fall der $d p_{2}{ }^{1 / d} d t=0$ Kurve. Diese Kurve verläuft relativ flach. Das bedeutet, daß die Umweltpolitik in Region 2 relativ schwach greift, schon in der Ausgangslage wird viel emittiert. Wird dann der Diffusionskoeffizient erhöht, steigen die Emissionen und die Steuer sinkt [eindeutige Effekte], jedoch sinken die Immissionen und 


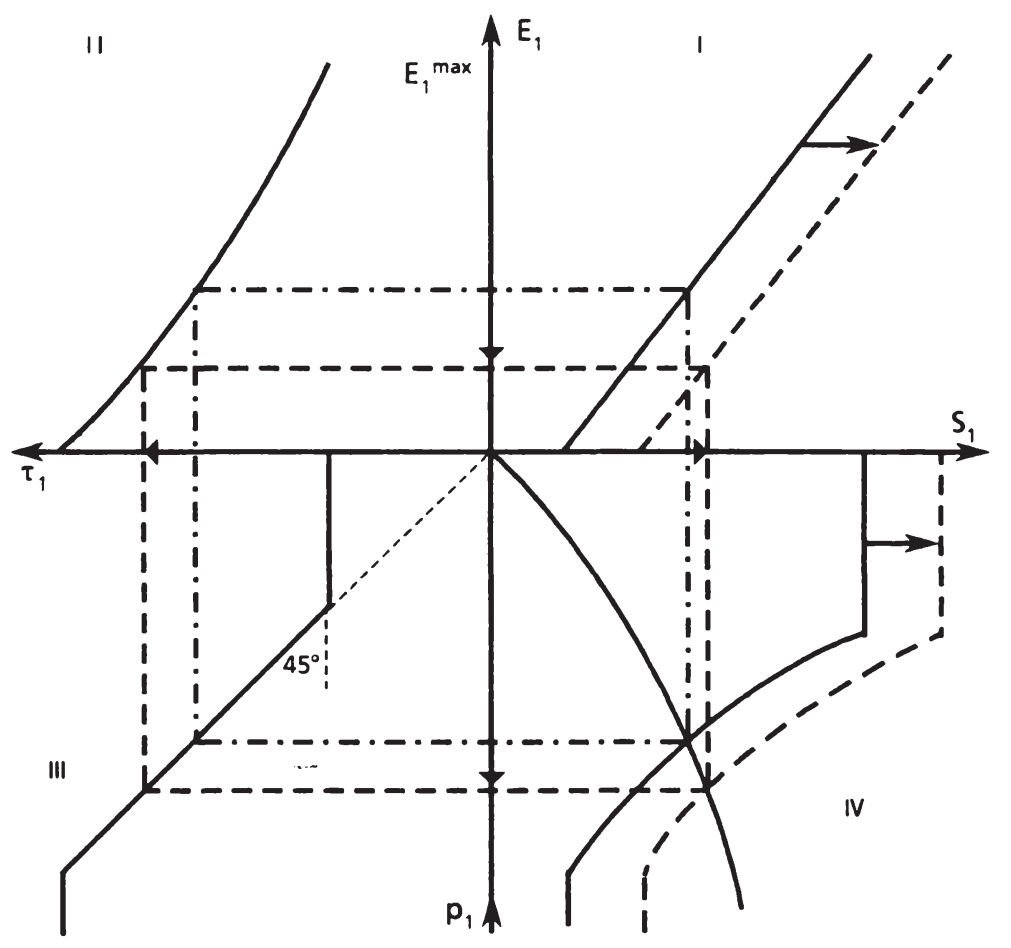

Schaubild 4.8: Änderungen in Region 1 bei erhöhtem Diffusionskoeffizienten

der Zukunftsschaden [ $p_{2}^{\infty}$ steigt] und der Konsum steigt. In der Region werden Wohlfahrtsgewinne realisiert. Im Fall der $d p_{2}{ }^{2} / d t=0$ Kurve sinkt zwar die Steuer ebenfalls und die Emissionen - und damit der Konsum steigen, jedoch steigen auch die Immissionen und der Zukunftsschaden. Hier kann jetzt keine eindeutige Aussage über die Wohlfahrtsänderung in Region 2 gemacht werden, da sich zwei entgegengesetzte Effekte gegenüberstehen: Durch die Immissionserhöhung ist ein negativer Wohlfahrtseffekt gegeben, während die Konsumsteigerung eine positive Wohlfahrtsänderung ergibt. Es kann also bei einer in der Ausgangslage strengen 


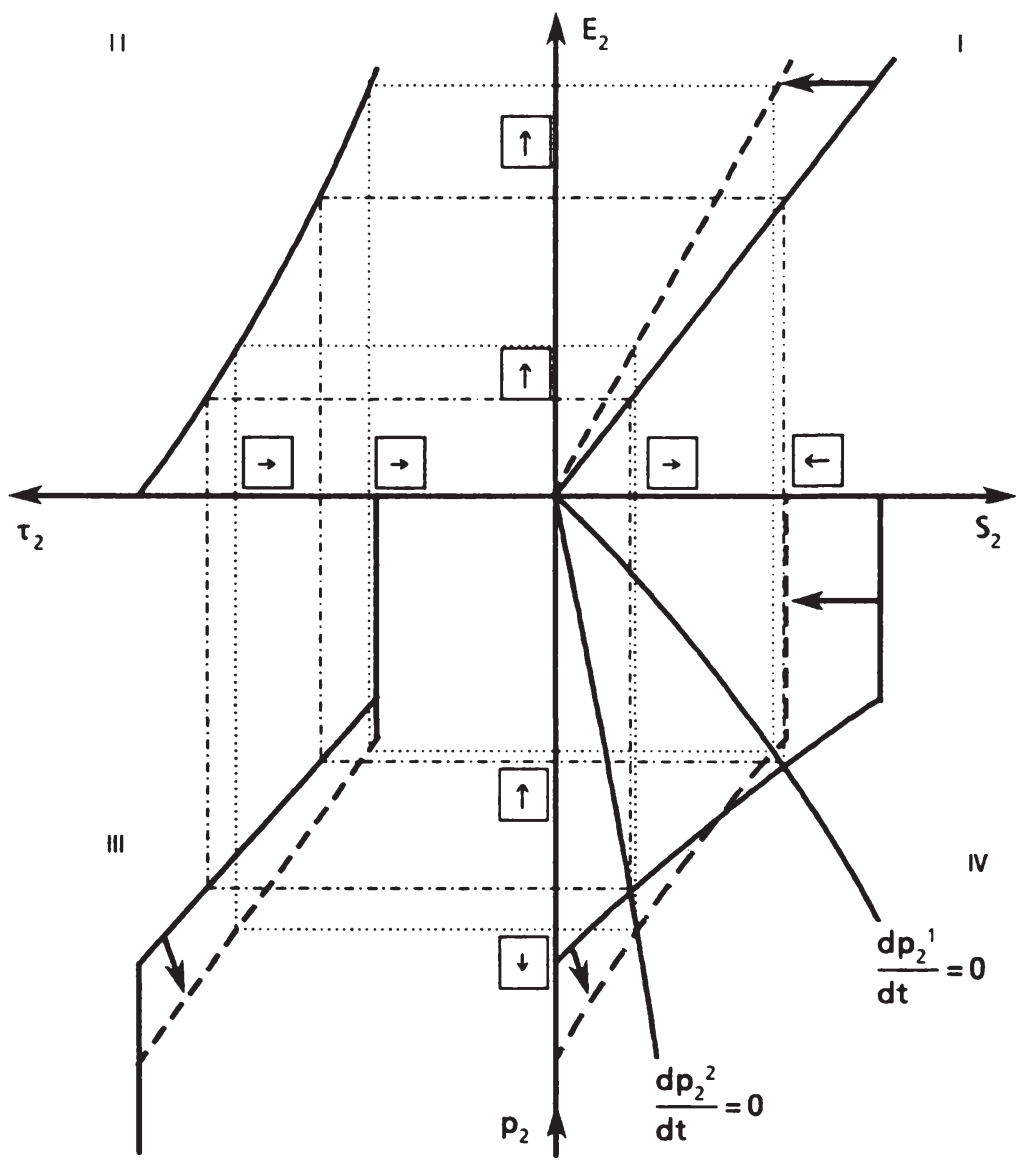

Schaubild 4.9: Die Effekte einer Diffusionskoeffizientenerhöhung auf Region 2

Umweltpolitik der Fall eintreten, daß durch eine Erhöhung des Diffusionskoeffizienten eine Wohlfahrtsminderung eintritt. 


\section{ZENTRALREGIERUNG}

\subsection{DAS OPTIMIERUNGSPROBLEM UND DIE OPTIMALBEDINGUNGEN}

Die Zentralbehörde sieht sich folgendem Optimierungsproblem gegenüber ${ }^{10)}$

$$
\begin{array}{cl}
\operatorname{Max} W= & \int_{0}^{\infty}\left|\sum_{k=1}^{2} \beta_{k} u^{k}\left(X_{k}, S_{k}\right)\right| e^{-\delta t} d t \\
\text { u.d.B. } & \text { (3.1) } \quad f\left(R_{i}, E_{i}\right)-X_{i} \geq 0 \\
& (3.2) \quad a_{i} R_{i}-E_{i} \geq 0 \\
& (4.3) \quad \mathbf{R}_{i}-R_{i} \geq 0 \\
& (4.1 . i i) \quad \frac{d S_{2}}{d t}=\left(1-\mathrm{Y}_{2}\right) E_{2}-a_{2} S_{2} \\
& (4.1 . \mathrm{i}) \quad \frac{d S_{1}}{d t}=E_{1}+\mathrm{Y}_{2} E_{2}-\mathrm{a}_{1} S_{1} \\
& X_{i}, R_{i}, E_{i} \geq 0 \quad \forall t \in[0, \infty), i=1,2 \\
& S_{i}(0)=S_{i}^{0} \text { gegeben } \\
& \delta \text { gegeben und positiv }
\end{array}
$$

Hierauf wird die Methode der Kontrolltheorie angewandt und man erhält folgende Lagrange-Funktion in laufenden Werten:

$$
\begin{aligned}
L=\sum_{k=1}^{2} B_{k}\left\{u^{k}\left(X_{k}, S_{k}\right)+\lambda^{k}{ }_{1}\left[f^{k}\left(R_{k}, E_{k}\right)-X_{k}\right]+\lambda^{k}{ }_{2}\left[a_{k} R_{k}-E_{k}\right]\right. \\
\left.+\lambda^{k}{ }_{3}\left[R_{k}-R_{k}\right]+\lambda^{k}{ }_{4} R_{k}+\lambda^{k}{ }_{5} E_{k}+\lambda^{k}{ }_{6} X_{k}\right\} \\
+B_{1} p_{1}\left[E_{1}+\gamma_{2} E_{2}-\alpha_{1} S_{1}\right]+B_{2} p_{2}\left[\left(1-\gamma_{2}\right) E_{2}-\alpha_{2} S_{2}\right]
\end{aligned}
$$

woraus sich folgende Bedingungen ergeben $(i=1,2)$ :

10) Vgl. Kapitel 3, Abschnitt 1.5.2. 
(i) $\quad L_{X i}=B_{i}\left\{u_{X}^{i}-\lambda^{i}{ }_{1}+\lambda_{6}^{i}\right\}=0$

$X_{i} \geq 0$

(ii) $L_{R i}=B_{i}\left\{\lambda^{i}{ }_{1} f_{R}+\lambda^{i}{ }_{2} a_{i}-\lambda^{i}{ }_{3}+\lambda^{i}{ }_{4}\right\}=0$

$R_{i} \geq 0$

(iii.1) $L_{E 1}=B_{1}\left\{\lambda^{1}{ }_{1} f_{E}{ }_{E}-\lambda^{1}{ }_{2}+\lambda^{1}{ }_{5}+p_{1}\right\}=0$

$E_{1} \geq 0$

(iii.2) $L_{E 2}=B_{2}\left\{\lambda^{2}{ }_{1} P_{E}-\lambda^{2}{ }_{2}+\lambda^{2}{ }_{5}+\left(1-\gamma_{2}\right) p_{2}\right\}+B_{1} \gamma_{2} p_{1}=0$

$E_{2} \geq 0$

(iv.1) $L_{p 1}=\beta_{1} \frac{d S_{1}}{d t}=\beta_{1}\left[E_{1}+Y_{2} E_{2}-a_{1} S_{1}\right]$

(iv.2) $L_{p 2}=\beta_{2} \frac{d S_{2}}{d t}=\beta_{2}\left[\left(1-\mathrm{Y}_{2}\right) E_{2}-a_{2} S_{2}\right]$

(4.26) (v) $\quad L_{S i}=\beta_{i}\left|-\frac{d p_{i}}{d t}+\delta p_{i}\right|=\beta_{i}\left[u_{S}^{i}-a_{i} p_{i}\right]$

(vi)

$$
B_{i} \lambda_{1}^{i}\left[f^{\prime}\left(R_{i}, E_{i}\right)-X_{i}\right]=0
$$

$\lambda_{1}^{i} \geq 0$

(vii)

$B_{i} \lambda_{2}^{i}\left[a_{i} R_{i}-E_{i}\right]=0$

$\lambda_{2}^{i} \geq 0$

(viii)

$\boldsymbol{B}_{i} \lambda^{i}{ }_{3}\left[\mathbf{R}_{i}-R_{i}\right]=0$

$\lambda_{3}^{i} \geq 0$

(ix)

$B_{i} \lambda^{i}{ }_{4} R_{i}=0$

$\lambda_{4}^{i} \geq 0$

(x)

$B_{i} \lambda^{i}{ }_{5} E_{i}=0$

$\lambda_{5}^{i} \geq 0$

(xi)

$B_{i} \lambda_{6}{ }_{6}{ } X_{i}=0$

$\lambda_{6}^{i} \geq 0$

Bezüglich der Nutzenfunktion gelten die gleichen Annahmen wie im autonomen Fall, so daß Vollbeschäftigung der Ressourcen und positiver Güterkonsum gilt. Auch die Besteuerungsfunktionen (4.8) und (4.9) gelten mit den für den autonomen Fall und im Grundmodell beschriebenen Eigenschaften. Die Zuordnung zwischen Steuer und Schattenpreis für Region 1, die emissionsimportierende Region, ist weiterhin durch (4.10) gegeben. Für Region 2 ergibt sich jedoch eine Änderung: 
(4.27)

$$
\tau_{2}=\left\{\begin{array}{l}
\tau_{2}^{\prime} \\
-\left(1-\gamma_{2}\right) p_{2}-\frac{B_{1} \gamma_{2}}{B_{2}} p_{1} \\
\tau_{2} "
\end{array}\right.
$$

für $p_{2} \geq p_{2}{ }^{\prime}$,

$$
\begin{aligned}
& \qquad p_{2}^{\prime}\left(p_{1}\right) \equiv \frac{-\tau_{2}^{\prime}}{1-\gamma_{2}}+\frac{B_{1} \gamma_{2}}{B_{2}\left(1-\gamma_{2}\right)} p_{1} \\
& \text { für } p_{2} \in\left(p_{2}^{\prime}, p_{2}^{\prime \prime}\right) \text {, }
\end{aligned}
$$

$$
p_{2}^{\prime \prime}\left(p_{1}\right) \equiv \frac{-\tau_{2} "}{1-\gamma_{2}}+\frac{B_{1} \gamma_{2}}{B_{2}\left(1-\gamma_{2}\right)} p_{1}
$$

für $p_{2} \leq p_{2} "$

Die Steuer in Region 2 ist jetzt also nicht mehr nur vom Zukunftsschaden in Region 2 abhängig, sondern es werden auch die Auswirkungen auf Region 1 berücksichtigt. Bei der folgenden Analyse wird davon ausgegangen, da $\beta$ beide Regionen gleich gewichtet sind, deshalb gilt $\left[B_{1} / B_{2}\right]=1$. In Schaubild 4.10 sind die Beziehungen zwischen der Steuer, den Schattenpreisen und den Emissionen für Region 2 dargestellt. Je höher die Schäden in Region 1 bewertet werden, desto weiter nach außen verschiebt sich die Steuergerade. Für einen Schattenpreis $\mathbf{p}_{2}$ sind die Steuern in Region 2 umso höher und die Emissionen umso niedriger, je höher die Schäden in Region 1 bewertet werden. 


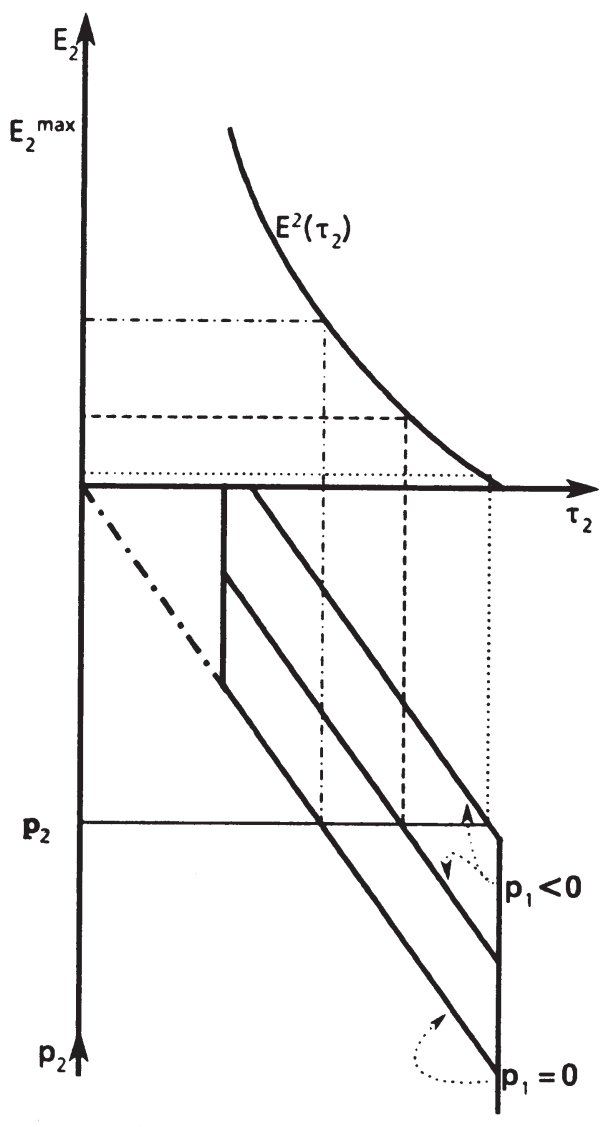

Schaubild 4.10: Die Beziehungen zwischen Steuern, Emissionen und Schattenpreisen der Region 2 bei einer Zentralregierung 


\subsection{DAS STEADY-STATE DER ÖKONOMIE}

Es wird jetzt unterstellt, daß eine innere Lösung existiert, es gilt also $E_{i} \epsilon$ $\left(0, E_{i}{ }^{\max }\right)(i=1,2)$. Dann ist $\lambda_{2}^{i}=\lambda_{5}^{i}=0$. Wir erhalten ein Differentialgleichungssystem:

$$
\begin{aligned}
& \text { (i) } \frac{d S_{1}}{d t}=E^{1}\left(\tau_{1}\right)+\mathrm{Y}_{2} E^{2}\left(\tau_{2}\right)-a_{1} S_{1} \\
& \text { (ii) } \frac{d p_{1}}{d t}=\left(\delta+a_{1}\right) p_{1}-u_{S}^{1} \\
& \text { (iii) } 0=\tau_{1}+p_{1} \\
& \text { (iv) } \frac{d S_{2}}{d t}=\left(1-\mathrm{\gamma}_{2}\right) E^{2}\left(\tau_{2}\right)-a_{2} S_{2} \\
& \text { (v) } \frac{d p_{2}}{d t}=\left(\delta+a_{2}\right) p_{2}-u_{S}^{2} \\
& \text { (vi) } 0=\tau_{2}+\left(1-\gamma_{2}\right) p_{2}+\gamma_{2} p_{1}
\end{aligned}
$$

Hier ist eine Aufspaltung wie im autonomen Fall nicht mehr möglich. Jedoch kann das Differentialgleichungssystem für gegebene Anfangsimmissionsbestände $S_{1}{ }^{0}$ und $S_{2}{ }^{0}$ sowie für geeignet gewählte Schattenpreise $p_{1}(0)$ und $p_{2}(0)$ gelöst werden [vgl. Hadley, $\operatorname{Kemp}(1971,370)$ ].

Zur Veranschaulichung der so erhaltenen Steady-State Lösung dienen die folgenden Überlegungen.

Für Region 1 ergeben sich die analogen Schlußfolgerungen wie beim autonomen Fall, wenn man sich jeweils die Emissionen aus Region 2 als gegeben vorgibt (vgl. hierzu Schaubild 4.4).

Betrachten wir jetzt Region 2 unter der Annahme, daß sie sich an die Emissionen der Region 1 jeweils anpassen müssen, da diese im Kalkül für Region 2 mit berücksichtigt werden müssen. Unter der Annahme, daß sich die Emissionen in Region 1 parametrisch ändern, ergibt sich für Region 2 folgendes Gleichungssystem für die Steady-State-Reaktionsfunktion: 
(4.29) $\left[\begin{array}{cccc}1-\gamma_{2} & -\alpha_{2} & 0 & 0 \\ 0 & -u_{S S}^{2} & \delta+\alpha_{2} & 0 \\ 0 & 0 & 1-\gamma_{2} & 1 \\ 1 & 0 & 0 & -E_{\tau}^{2}\end{array}\right]\left[\begin{array}{c}\frac{d E_{2}^{*}}{d E_{1}} \\ \frac{d S_{2}^{*}}{d E_{1}} \\ \frac{d p_{2}^{*}}{d E_{1}} \\ \frac{d \tau_{2}^{*}}{d E_{1}}\end{array}\right]=\left[\begin{array}{c}0 \\ 0 \\ \\ \frac{\underline{\gamma}_{2}}{E^{1}} \\ \tau \\ 0\end{array}\right]$

Mit der Systemdeterminanten

$$
\Delta_{2}=\left(1-\gamma_{2}\right) E^{2}{ }_{\tau} u_{S S}^{2}+\alpha_{2}\left(\delta+\alpha_{2}\right)>0
$$

Hieraus ergeben sich folgende Abhängigkeiten der Steady-State-Werte der Region 2 von den Emissionen der Region 1:

$$
\begin{aligned}
& \frac{d E_{2}^{*}}{d E_{1}}=\frac{a_{2}\left(\delta+a_{2}\right) \mathrm{Y}_{2} E_{\tau}^{2}}{E_{\tau}^{1} \Delta_{2}}>0 \\
& \frac{d S_{2}^{*}}{d E_{1}}=\frac{\left(\delta+a_{2}\right) \mathrm{Y}_{2}\left(1-\mathrm{Y}_{2}\right) E_{\tau}^{2}}{E_{\tau}^{1} \Delta_{2}}>0 \\
& \frac{d p_{2}^{*}}{d E_{1}}=\frac{\mathrm{Y}_{2}\left(1-\mathrm{Y}_{2}\right) E_{\tau}^{2} u_{\mathrm{SS}}^{2}}{E_{\tau}^{1} \Delta_{2}}<0 \\
& \frac{d \tau_{2}^{*}}{d E_{1}}=\frac{a_{2}\left(\delta+\mathrm{a}_{2}\right) \mathrm{Y}_{2}}{E_{\tau}^{1} \Delta_{2}}<0
\end{aligned}
$$

Schaubild 4.11 zeigt die Anpassungen.

Eine Veränderung gegenüber dem autonomen Fall ist im III. Quadranten zu sehen. Durch die Berücksichtigung der durch die Emissionen aus Region 2 verursachten Zukunftsschäden in Region 1 verschiebt sich die Steuergerade parallel nach außen, je höher die Zukunftsschäden in Region 1 be- 


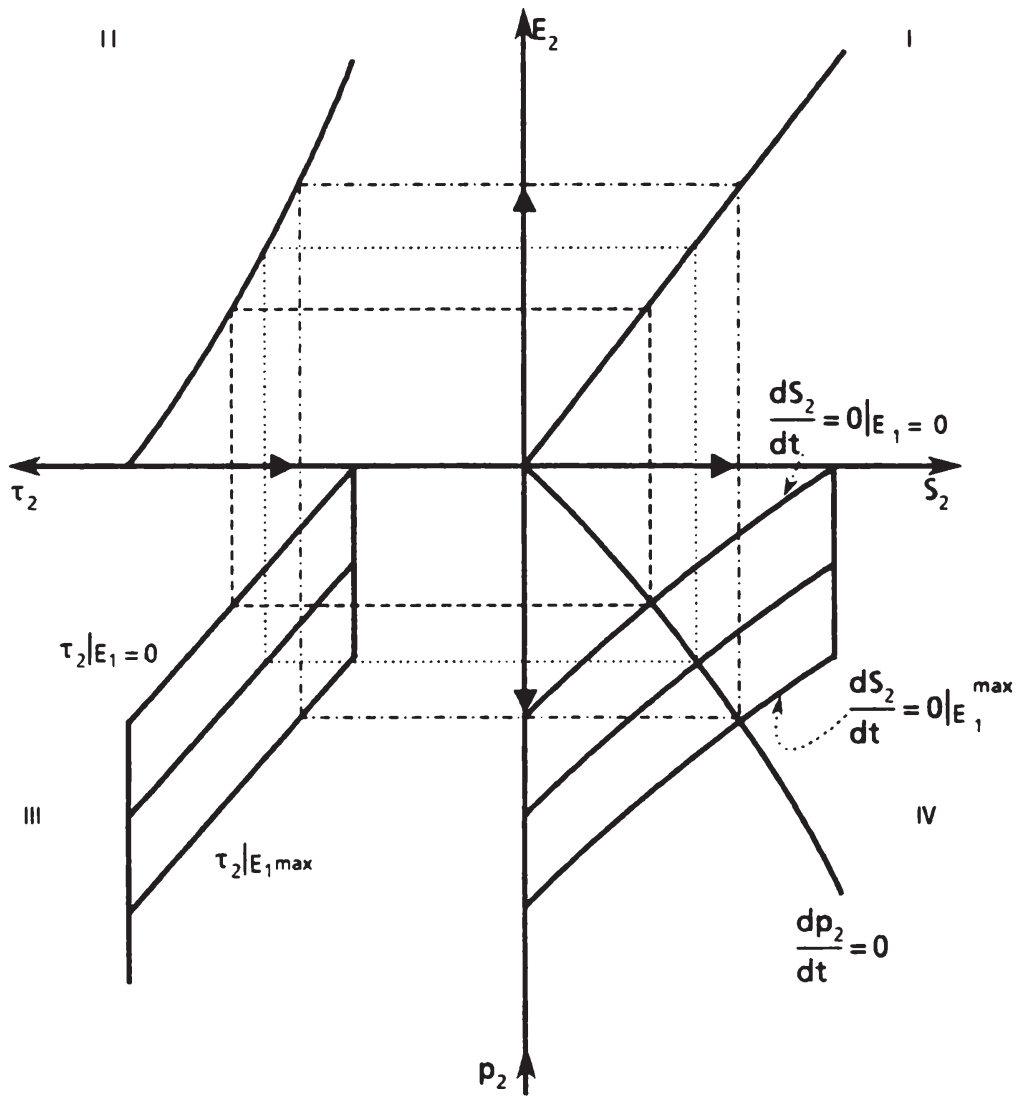

Schaubild 4.11: Steigendes $E_{1}$ für Region 2

wertet werden. Damit ist indirekt eine Beziehung zwischen den produzierten Emissionen in Region 1 und den Emissionen in Region 2 hergestellt. Im IV. Quadranten verschiebt sich entsprechend die $d S_{2} / d t=0$ Kurve, während die $d p_{2} / d t=0$ Kurve liegen bleibt. Gehen wir von steigenden Emissionen in Region 1 aus: Ist $E_{1}=0$, der Zukunftsschaden aus einer Emissionseinheit wird in Region 1 sehr hoch bewertet, dann sollen auch 
die Emissionen in Region 2 niedrig sein, da ja ein Teil in die andere Region exportiert wird. Steigt jetzt $E_{1}$, dann steigen auch die Optimalwerte der Immissionen und die Optimalwerte der Schattenpreise sinken. Gleichzeitig sinkt auch die optimale Steuer und deshalb erhöhen sich die Optimalwerte der Emissionen (vgl. die Pfeile in Schaubild 4.11).

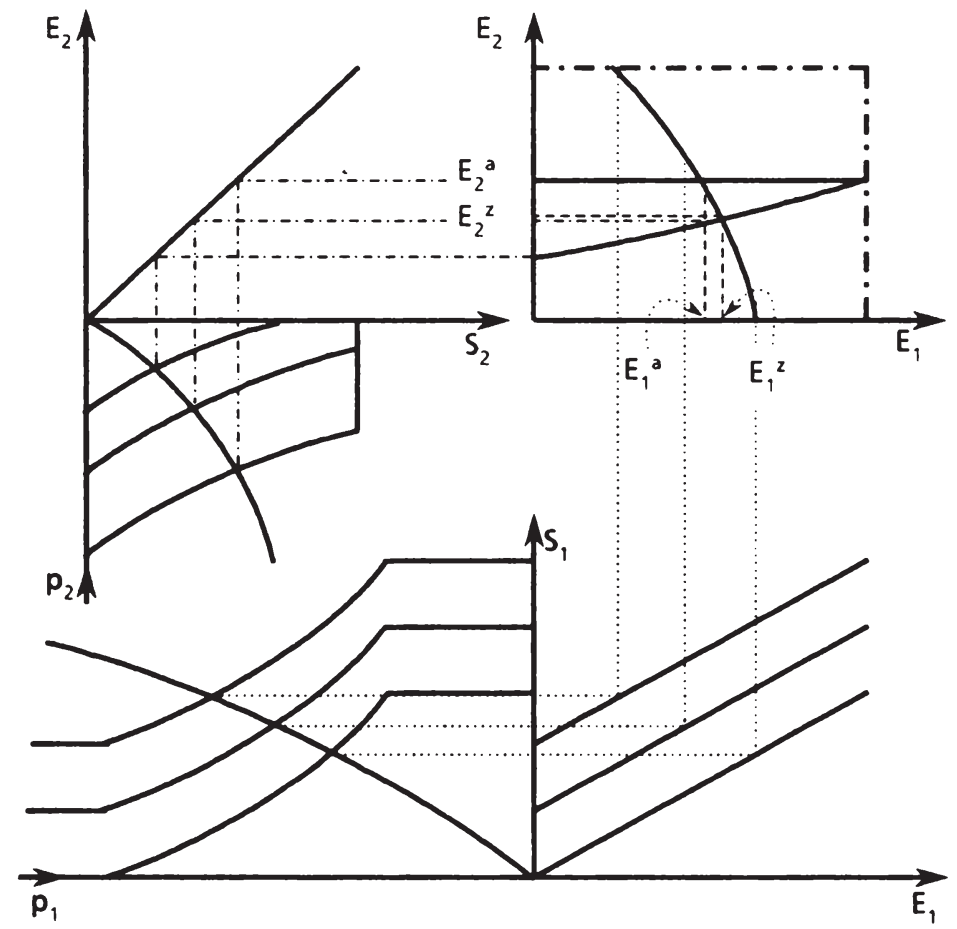

Schaubild 4.12: Die Ableitung der Steady-State Reaktionsfunktionen

Man kann hier also Steady-State-Reaktionsfunktionen ableiten, die unterschiedlichen Werten von $E_{1}$ die jeweils optimalen Werte von $E_{2}$ zuordnen. Für Region 1 gilt weiterhin die im autonomen Fall abgeleitete Steady-State-Reaktionsfunktion. Graphisch läßt sich die Funktion für Region 2 wie in Schaubild 4.12 dargestellt ableiten.

Die Emissionsreaktionsfunktion für Region 2 hat positive Steigung da 
durch die Berücksichtigung der Schäden in Region 1 bei steigenden Emissionen in 1 sich die Produktionsmöglichkeiten in Region 2 erhöhen. Sie verläuft jedoch jeweils unterhalb der Vertikalen, die für den autonomen Fall gilt. Nur wenn die Zukunftsschäden in Region 1 nicht berücksichtigt werden, sind die optimalen Emissionen der Region 2 im autonomen und im "zentralregierungs"-Fall gleich.

\subsection{DIE AUSWIRKUNGEN VON PARAMETERÄNDERUNGEN}

Betrachten wir auch hier die Änderungen der das Steady-State mitbestimmenden Parameter. Die Änderungsrichtungen sind in Tabelle 4.2 zusammengefaßt. Eine analytische Ableitung erfolgt im Anhang II zu diesem Kapitel.

\begin{tabular}{|c|c|c|c|c|c|c|c|c|c|c|}
\hline & $d E_{1}$ & $d S_{1}$ & $d p_{1}$ & $d \tau_{1}$ & $d X_{1}$ & $d E_{2}$ & $d S_{2}$ & $d p_{2}$ & $d \tau_{2}$ & $d X_{2}$ \\
\hline$d \delta$ & $?$ & $\oplus$ & $?$ & $?$ & $?$ & $\oplus$ & $\oplus$ & $?$ & $\Theta$ & $\oplus$ \\
\hline$d \alpha_{1}$ & $\oplus$ & $?$ & $\oplus$ & $\Theta$ & $\oplus$ & $\oplus$ & $\oplus$ & $\Theta$ & $\Theta$ & $\oplus$ \\
\hline$d \mathbf{R}_{1}$ & $\oplus$ & $\oplus$ & $\Theta$ & $\oplus$ & $\oplus$ & $\Theta$ & $\Theta$ & $\oplus$ & $\oplus$ & $\Theta$ \\
\hline$d \alpha_{2}$ & $\Theta$ & $\oplus$ & $\Theta$ & $\oplus$ & $\Theta$ & $\oplus$ & $?$ & $\oplus$ & $\Theta$ & $\oplus$ \\
\hline$d \mathbf{R}_{2}$ & $\Theta$ & $\oplus$ & $\Theta$ & $\oplus$ & $\Theta$ & $\oplus$ & $\oplus$ & $\Theta$ & $\oplus$ & $\oplus$ \\
\hline$d \gamma_{2}$ & $?$ & $?$ & $?$ & $?$ & $?$ & $?$ & $\Theta$ & $\oplus$ & $?$ & $?$ \\
\hline$\left(p_{2}>p_{1}\right)$ & & & & &
\end{tabular}

Tabelle 4.2: Auswirkungen von Parameteränderungen bei einer Zentralregierung

\subsubsection{Die Abhängigkeit von der sozialen Diskontrate}

Für Region 2 sind die Auswirkungen der sozialen Diskontrate weitgehend identisch mit denen im autonomen Fall: Je größer $\delta$ ist, umso größer sind die Emissionen, die Immissionen und die Produktion, während die Steuer umso niedriger ist. Die Auswirkungen auf den Schattenpreis sind jedoch nicht eindeutig. Ob der Schattenpreis der Region 2 sinkt oder steigt, hängt von den Auswirkungen der Änderung der Diskontrate auf den Schattenpreis der Region 1 ab. Es gilt 


$$
\begin{gathered}
\frac{d p_{2}}{d \delta} \geq 0 \Leftrightarrow \frac{a_{1} p_{1}}{Y_{2}^{2} E_{\tau}^{2} u_{S S}^{1}+\Delta_{1}} \geq \frac{a_{2} p_{2}}{Y_{2}\left(1-Y_{2}\right)^{2} E_{\tau}^{2} u_{S S}^{2}} \Leftrightarrow \\
\left|\left(\left.\frac{d p_{1}}{d \delta}\right|_{d p_{2}=0}\right)^{1}\right| \geq\left|\left(\left.\frac{d p_{1}}{d \delta}\right|_{d p_{2}=0}\right)^{2}\right|
\end{gathered}
$$

wobei die linke Seite die Änderungen des Schattenpreises der Region 1 über die Änderungen in Region 1 angibt, während die rechte Seite die Änderung des Schadens in Region 1 über Änderungen in Region 2 angibt. Sind die Auswirkungen einer Diskontratenänderung in Region 1 auf den Schaden in Region 1 größer (kleiner) als die Auswirkungen von Region 2, dann sinkt (steigt) der Schattenpreis in Region 2. Hier kommt zum Ausdruck, daß die Bewertung des Schadens in Region 2 entscheidend von der Bewertung des Schadens in Region 1 abhängt. Im Gegensatz zu den autonomen Regionen (der Zukunftsschaden für Region 2 ist bei einer höheren Diskontrate gesunken), kommen hier die Interdependenzen, die von der Zentralregierung berücksichtigt werden, zum Ausdruck.

Für Region 1 sind die Auswirkungen einer Änderung der Diskontrate die gleichen wie im autonomen Fall ${ }^{11}$ :

$$
\begin{aligned}
& \frac{d E_{1}}{d \delta} \geq_{0} \Leftrightarrow \frac{d p_{1}}{d \delta} \geq 0 \Leftrightarrow \frac{d \tau_{1}}{d \delta} \leq_{0} \Leftrightarrow \frac{d X_{1}}{d \delta} \geq_{0} \Leftrightarrow \\
& \left.\frac{d S_{1}}{d E_{2}}\right|_{d E_{1}=0} E_{6}^{2 \mathrm{a}}-\left.\frac{d S_{1}}{d \delta}\right|_{d p_{1}=0} \geq_{0}
\end{aligned}
$$

wobei $E^{2 \mathrm{a}} \delta$ die Erhöhung der Emissionen in Region 2 angibt, die sich auch für autonome Regionen ergibt (bei gleichem Ausgangspunkt).

10) Wobei $\Delta_{1}$ die im Anhang 4.I definierte Determinante für den autonomen Fall der Region 1 darstellt.

11) Vgl. Abschnitt 2.4.2. 


\subsubsection{Die Abhängigkeit von den Assimilationskoeffizienten}

Je größer der Assimilationskoeffizient in Region 1 ist, desto mehr kann sowohl in Region 1 als auch in Region 2 emittiert werden und damit auch mehr produziert und konsumiert werden. Die optimalen Steuern in Region 1 als auch in Region 2 sind umso niedriger, umso höher $\alpha_{1}$ ist. Während jedoch in Region 1 der bewertete Zukunftsschaden umso niedriger wird, ist er in Region 2 umso höher, da dort die Immissionen steigen. Über die Auswirkungen auf die Immissionen in Region 1 kann wiederum keine eindeutige Aussage gemacht werden, es sind wieder die beiden Effekte zu beobachten, die sich schon im Exkurs in Kapitel 3 ergaben, jedoch ist hier zusätzlich noch die Auswirkung des erhöhten Emissionsimports zu berücksichtigen:

$$
\frac{d S_{1}}{d \mathrm{a}_{1}} \geq 0 \Leftrightarrow\left[\frac{d E_{1}}{d \mathrm{c}_{1}} \frac{d \tau_{1}}{d p_{1}}+\mathrm{Y}_{2} \frac{d E_{2}}{d p_{1}}\left|\frac{d p_{1}}{d \mathrm{a}_{1}}\right|_{d S_{1}=0} \geq\left.\frac{d E_{1}}{d \mathrm{a}_{1}}\right|_{d S_{1}=d E_{2}=0}\right.
$$

wobei die rechte Seite äquivalent ist zu dem Effekt in Bedingung (3.36). Dieser Effekt wird auch hier reiner Emissionseffekt genannt, der nur über das Ökosystem durch eine Veränderung der Emissionen wirkt, während die linke Seite die Bewertung des Schadens ausdrückt.

Für die Änderung des Assimilationskoeffizienten in Region 2 erhalten wir äquivalente Ergebnisse zu denen in Region 1, die sich darüberhinaus auch bei der autonomen Optimierung der Richtung nach ergaben. Die Emissionen in Region 2 steigen, die Schattenpreise sinken, die Steuer sinkt und die Produktion steigt. Die Änderung der Immissionen setzt sich wieder aus zwei Effekten zusammen: Einmal die Änderung der Bewertung des Schadens über die Verschiebung der $d p_{2} / d t=0$ Kurve bei konstanten Immissionen und zum anderen die Änderung der Bewertung des Schadens über die Änderung der Emissionen bei konstanten Immissionen (vgl. die Ausführungen in Abschnitt 2.4.2 dieses Kapitels):

$$
\frac{d S_{2}}{d a_{2}} \geq\left. 0 \Leftrightarrow \frac{d p_{2}}{d a_{2}}\right|_{d S_{2}=0}<\left.\frac{d p_{2}}{d E_{2}} \frac{d E_{2}}{d a_{2}}\right|_{d S_{2}=0}
$$


Ob die Immissionen in Region 2 zu- oder abnehmen ist also auch bei einer Zentralregierung nicht direkt abhängig von den Auswirkungen des Emissionsexportes nach Region 1 . Eine indirekte Abhängigkeit ergibt sich jedoch über die verminderte Emissionszunahme, da (bei identischem Ausgangspunkt)

$$
\left.\frac{d E_{2}}{d \mathrm{a}_{2}}\right|^{a}>\left.\frac{d E_{2}}{d \mathrm{a}_{2}}\right|^{2}
$$

gilt.

\subsubsection{Die Abhängigkeit von den Ressourcenausstattungen}

Hier hat die Ressourcenausstattung der Region 1 auch Auswirkungen auf die Umweltpolitik in Region 2. Allgemein läßt sich sagen, daß je höher die Ressourcenausstattung in Region 1 ist, desto weniger Emissionen werden in Region 2 produziert. Diesen Sachverhalt gilt es weiter zu untersuchen. Es überrascht, daß bei höheren Emissionen in Region 1 die Emissionen in Region 2 abnehmen. Jedoch wird durch den Ressourcenbestand die Bewertung des Schadens in Region 1 beeinflußt. Je größer der Faktorbestand in Region 1 ist, umso höher wird der Schaden bewertet, der aus der Produktionstätigkeit entsteht, d. h. der Schatten preis $p_{1}$ wird für konstante Emissionen größer. Dies liegt daran, daß die Grenzproduktivität der Emissionen bei höherem Ressourcenbestand steigt und damit auch die Steuer für ein bestimmtes Emissionsniveau. Damit wird aber die Bewertung des Schadens einer Emissionseinheit höher [vgl. die Gleichungen (4.8) und (4.9)]. Je größer also der Ressourcenbestand in Region 1 ist, desto größer sind in Region 1 die Emissionen, die Immissionen und die Bewertung des Schadens, die Steuer und die Produktion und desto kleiner sind in Region 2 die Emissionen, die Immissionen und die Bewertung des Schadens, die Steuer steigt und die Produktion sinkt.

Je größer der Ressourcenbestand in Region 2 ist, desto größer sind dort die Emissionen, die Immissionen und die Bewertung des Schadens, sowie die Steuer und die Produktion; in Region 1 sind die Emissionen und die Pro- 
duktion umso niedriger, während die Immissionen und die Bewertung des Schadens sowie die Steuer umso höher sind.

Daß sich die Grenzproduktivität der Emissionen bei größerem Ressourcenbestand (und Vollbeschäftigung) erhöht, ist hier entscheidend für die Ableitung der obigen Ergebnisse.

\subsubsection{Die Erhöhung des Diffusionskoeffizienten}

Zur Analyse einer Änderung des Diffusionskoeffizienten müssen die Regionen näher spezifiziert werden, da das Verhältnis der Zukunftsschäden in beiden Regionen zueinander die Änderung der Steady-State-Werte bestimmt. Es wird davon ausgegangen, daß die Präferenzen - und damit die Nutzenfunktionen - und die Produktionstechnologien identisch sind. Weiterhin seien die Assimilationskoeffizienten in den beiden Regionen identisch. Dann ist der Schattenpreis der Immissionen in Region 2 größer als der Schattenpreis in Region ${ }^{12)}$.

Ein Vergleich der Änderungen im Zentralregierungsfall mit dem autonomen Fall zeigt, daß sich die Eindeutigkeiten genau umkehren: Die Immissionen in Region 2 und die Bewertung des Schadens daraus in Region 2 sind umso kleiner, je größer der Diffusionskoeffizient ist, während über die Richtung der Änderungen der anderen Variablen keine eindeutige Aussage gemacht werden kann.

Untersuchen wir zuerst die Auswirkungen in Region 2. Es gilt

$$
\begin{aligned}
& \frac{d E_{2}}{d \mathrm{Y}_{2}} \geq 0 \Leftrightarrow \frac{d \mathrm{\tau}_{2}}{d \mathrm{Y}_{2}}>0 \Leftrightarrow \frac{d X_{2}}{d \mathrm{Y}_{2}} \geq 0 \Leftrightarrow \\
& \left.\left|p_{2}\right| \frac{d p_{2}}{d E_{2}}\right|_{d \mathrm{Y}_{2}=0} \frac{E_{2}}{p_{2}}+1|| \leq\left.\left|p_{1}\right| \frac{d p_{1}}{d E_{2}}\right|_{d \mathrm{~V}_{2}=0} \frac{E_{2}}{p_{1}}+1||
\end{aligned}
$$

Ob die Emissionen in Region 2 bei einer Erhöhung des Diffusionskoeffizi-

12) Vgl. Anhang 4.II. 
enten zu- oder abnehmen ist abhängig davon, ob der Zukunftsschaden in Region 1 durch eine Erhöhung der Emissionen in Region 2 (bei konstantem $\gamma_{2}$ ) stärker oder schwächer zunimmt als in Region 2. Sind die Auswirkungen auf Region 2 größer als auf Region 1, dann steigen auch die Emissionen in Region 2 während die Steuer in Region 2 sinkt. Sind jedoch die Auswirkungen auf Region 1 größer als auf Region 2, steigt die Steuer in Region 2 und die Emissionen sinken.

Für Region 1 erhalten wir folgende Bedingungen:

$$
\frac{d E_{1}}{d \mathrm{Y}_{2}} \geq_{0} \Leftrightarrow \frac{d S_{1}}{d \mathrm{Y}_{2}} \leq_{0} \Leftrightarrow \frac{d p_{1}}{d \mathrm{Y}_{2}} \geq 0 \Leftrightarrow \frac{d \tau_{1}}{d \mathrm{Y}_{2}} \leq 0 \Leftrightarrow \frac{d X_{1}}{d \mathrm{Y}_{2}} \geq 0 \Leftrightarrow
$$

$$
\frac{d E_{2} \mathrm{Y}_{2}}{d \mathrm{Y}_{2}} \frac{E}{2}_{E_{2}}>-1
$$

Die Änderungsrichtung der Emissionen, der Produktion, der Immissionen, der Bewertung des Schadens und der Steuer in Region 1 ist abhängig von der Emissionselastizität bezüglich des Diffusionskoeffizienten in Region 2. Sinken die Emissionen in Region 2 überproportional, dann wird in Region 1 ein Wohlfahrtsgewinn verzeichnet. Sinken die Emissionen in Region 2 nur unterproportional oder steigen gar, dann sinken die Emissionen in Region 1 und auch die Produktion, während die Immissionen und die Steuer sowie die Bewertung des Schadens steigen.

Die hier abgeleiteten Aussagen sind beispielhaft für bis auf die Diffusionsbeziehungen identische Regionen diskutiert worden. Diese Aussagen gelten jedoch allgemein, wenn die Beziehung $p_{1}<p_{2}$ gilt.

Ist dies nicht der Fall, gilt also $p_{1}>p_{2}$ (die Bewertung des Schadens in Region 1 ist geringer als in Region 2), dann sind die Auswirkungen auf Region 1 eindeutig: Die Emissionen und die Produktion sinken, während die Immissionen, die Steuer und der Schaden steigen. Für Region 2 sind dann im Gegensatz zum oben diskutierten Fall, alle Auswirkungen einer Diffusionskoeffizientenerhöhung nicht mehr eindeutig. Jedoch gilt Bedingung (4.33) weiterhin und für die Änderung der Immissionen und des Schattenpreises in Region 2 kann eine äquivalente Beziehung zu Bedingung (4.34) 
hergeleitet werden:

$$
\frac{d E_{2}}{d Y_{2}} \frac{1-Y_{2}}{E_{2}} \gtrless_{1} 1
$$

Steigen die verbleibenden Emissionen in Region 2 über(unter)proportional, dann steigen (sinken) die Immissionen und der Schattenpreis in Region 2. 


\section{AUTONOME REGIONEN MIT ZENTRALER STANDARDSETZUNG}

Im vorigen Abschnitt des Kapitels waren die Regionen nicht mehr eigenständig, sondern von einer Zentralregierung gesteuert und konnten nicht selbst über ihre Ziele bestimmen. Es kann jedoch aus historischen Gründen oder aus Gründen der parlamentarischen Demokratie (regionale Präferenzen) geboten sein, eigenständige Regionen zu erhalten. Eine Zentralregierung hat dann die Möglichkeit über die Setzung von Standards in die Umweltpolitik der Regionen einzugreifen. Die Regionen sind weiterhin relativ selbständig und die Zentralregierung greift ein, um etwaiges "Freifahrer"-Verhalten einer Region zu verhindern. Zu fragen ist dann, ob ein äquivalentes Ergebnis erzielt werden kann wie bei einer Zentralregierung und welche Änderungen sich ergeben.

\subsection{DIE OPTIMIERUNGPROBLEME}

Das Optimierungsproblem von Region 1 ändert sich gegenüber demjenigen im autonomen Fall nicht, da von der Annahme ausgegangen wird, da $B$ die Zentralregierung nur interregionale spill-overs angemessen berücksichtigt sehen will und die Regionen ansonsten ihre Eigenständigkeit behalten. Die Regionalbehörde der Region 1 nimmt weiterhin die Emissionen aus Region 2 als gegeben an.

Für Region 2 ergeben sich folgende Änderungen:

Wir gehen davon aus, daß die Zentralregierung per Verordnung bzw. per "Bundesgesetz" die Emissionen in Region 2 begrenzt. Sie gibt einen absoluten Emissionsstandard vor, den die Produzenten in Region 2 nicht überschreiten dürfen ${ }^{14)}$.

Es muß gelten

$$
E_{2}(t) \leq \mathbf{E}_{2} \quad\left(<E_{2}^{\max }\right) \quad \forall t \in[0, \infty)
$$

14) Vgl. Dudenhöffer (1984), der sich ausführlich mit der Regulierungsproblematik im Umweltbereich, allerdings in statischem Kontext, beschăftigt. 
(4.36) ersetzt also die Bedingung (3.2) beim Maximierungsproblem der Region 2. M. a. W. bei der Produktion in Region 2 kann bei Vollbeschäftigung die Kuppelproduktionsgrenze nicht mehr voll ausgeschöpft werden. Die Regionalregierung in Region 2 sieht sich dann folgendem Optimierungsproblem gegenüber:

$$
\begin{array}{ll}
\operatorname{Max} W_{2}=\int_{0}^{\infty} u^{2}\left(X_{2}, S_{2}\right) e^{-\delta t} d t \\
\text { u.d.B. (3.1) } \quad f^{2}\left(R_{2}, E_{2}\right)-X_{2} \geq 0 \\
\text { (4.36) } \quad E_{2}-E_{2} \geq 0 \\
\text { (4.3) } \quad R_{2}-R_{2} \geq 0 \\
\text { (4.1.ii) } \quad \frac{d S_{2}}{d t}=\left(1-\mathrm{x}_{2}\right) E_{2}-a_{2} S_{2} \\
\\
X_{2}, R_{2}, E_{2} \geq 0 \forall t \in[0, \infty) \\
& S_{2}(0)=S_{2}{ }^{0} \text { gegeben } \\
& \delta \text { gegeben und positiv }
\end{array}
$$

Für die Lagrange-Funktion in laufenden Werten ergibt sich also

$$
\begin{aligned}
L^{2}=u^{2}\left(X_{2}, S_{2}\right) & +p_{2}\left[\left(1-\gamma_{2}\right) E_{2}-\alpha_{2} S_{2}\right] \\
& +\lambda^{2}{ }_{1}\left[\rho^{2}\left(R_{2}, E_{2}\right)-X_{2}\right]+\lambda^{2}{ }_{2}\left[E_{2}-E_{2}\right] \\
& +\lambda^{2}{ }_{3}\left[R_{2}-R_{2}\right]+\lambda^{2}{ }_{4} R_{2}+\lambda^{2}{ }_{5} E_{2}+\lambda^{2}{ }_{6} X_{2}
\end{aligned}
$$

woraus sich folgende Bedingungen abgeleitet werden:

$$
L^{2}{ }_{X}=u^{2}{ }_{X}-\lambda^{2}{ }_{1}+\lambda^{2}{ }_{6}=0
$$$$
x_{2} \geq 0
$$

$$
L^{2}{ }_{R}=\lambda^{2}{ }_{1} f_{R}-\lambda^{2}{ }_{3}+\lambda^{2}{ }_{4}=0
$$$$
R_{2} \geq 0
$$

$$
L_{E}^{2}=\left(1-\gamma_{2}\right) p_{2}+\lambda^{2}{ }_{1} P_{E}-\lambda^{2}{ }_{2}+\lambda^{2}{ }_{5}=0
$$$$
E_{2} \geq 0
$$

(iv)

$$
L_{p}^{2}=\frac{d S_{2}}{d t}=\left(1-\mathrm{Y}_{2}\right) E_{2}-a_{2} S_{2}
$$




$$
L_{S}^{2}=-\frac{d p_{2}}{d t}+\delta p_{2}=u_{S}^{2}-a_{2} p_{2}
$$

$$
\lambda^{2}{ }_{1}\left[f^{2}\left(R_{2}, E_{2}\right)-X_{2}\right]=0
$$$$
\lambda^{2}{ }_{1} \geq 0
$$$$
\lambda_{2}^{2}\left[E_{2}-E_{2}\right]=0
$$$$
\lambda^{2}{ }_{2} \geq 0
$$$$
\lambda^{2}{ }_{3}\left[\mathbf{R}_{2}-R_{2}\right]=0
$$$$
\lambda^{2}{ }_{3} \geq 0
$$$$
\lambda^{2}{ }_{4} R_{2}=0
$$$$
\lambda^{2}{ }_{4} \geq 0
$$$$
\lambda^{2}{ }_{5} E_{2}=0
$$$$
\lambda^{2}{ }_{5} \geq 0
$$$$
\lambda^{2}{ }_{6} X_{2}=0
$$$$
\lambda_{6}^{2} \geq 0
$$

Aus der Wohlfahrtsfunktion folgt wiederum, daß positiver Güterkonsum vorliegen soll: $X_{2}>0$, d. h. $R_{2}>0$ und damit $\lambda^{2}{ }_{4}=\lambda_{6}^{2}=0$. Daraus folgt wieder, daß in der Region Vollbeschäftigung herrscht. Die Überlegungen zur Besteuerungsfunktion (4.8) bzw. (4.9) gelten auch hier, jedoch ändert sich der Definitionsraum an seiner unteren Grenze:

$$
\tau_{2}=u^{2}{ }^{2} P_{E}{ }_{E} \mid E_{2}=\mathbf{E}_{2}
$$

Auch die Beziehungen zwischen dem Schattenpreis $p_{2}$ und der optimalen Steuer $\tau_{2}$ bleiben im Prinzip erhalten bis auf die Begrenzungen:

(4.40) $\tau_{2}=\left\{\begin{array}{lll}\tau_{2} & \text { für } p_{2} \geq p_{2}, & \mathbf{p}_{2} \equiv\left[\left(-\tau_{2}\right) /\left(1-\gamma_{2}\right)\right] \\ -\left(1-\gamma_{2}\right) p_{2} & \text { für } p_{2} \in\left(\mathbf{p}_{2}, p_{2}{ }^{\prime \prime}\right) & p_{2}{ }^{"} \equiv\left[\left(-\tau_{2}{ }^{\prime \prime}\right) /\left(1-\gamma_{2}\right)\right] \\ \tau_{2}{ }^{\prime} & \text { für } p_{2} \leq p_{2}{ }^{\prime \prime} & \end{array}\right.$

Im folgenden wollen wir davon ausgehen, daß der Standard im SteadyState für Region 2 bindend ist. Ist er nicht bindend, dann ändert sich an der Analyse von Abschnitt 2 nichts.

Für die Beziehung zwischen Steuer und Zukunftsschaden gilt bei bindendem Emissionsstandard

$$
\tau_{2}=-\left(1-\gamma_{2}\right) p_{2}+\lambda^{2}
$$


Die Steuer ist aus zwei Teilen zusammengesetzt. Einmal drückt sie den Schaden aus, der aus der Emissionstätigkeit entsteht und zum anderen wird sie erhöht durch die Einhaltung des Emissionsstandards. Die Steuer ist höher als die sozialen Kosten, die aus der Emissionstätigkeit in Region 2 entstehen. Es kann in Region 2 weniger konsumiert werden als bei regionenegoistischer Optimierung (vgl. Abschnitt 2). Durch die Standardsetzung muß also Konsumverzicht geübt werden, um die sozialen Kosten aus der Emissionstätigkeit in Region 2, die außerhalb dieser Region entstehen, angemessen zu berücksichtigen.

Anhand des Schaubildes 4.13 wird die Analyse vorgenommen. Im III. Quadranten ist die Aufspaltung der Emissionssteuer in Zukunftsschaden $p_{2}{ }^{\infty}$ und die Kosten für den Konsumverzicht $\lambda_{2}^{2}{ }_{2}^{\infty}$ dargestellt. Die optimale Steady-State-Steuer $\boldsymbol{t}_{2}$ würde ohne Emissionsstandard einem Zukunftsschaden von $\mathbf{p}_{2}{ }^{\infty}$ in Region 2 entsprechen. Da durch die Begrenzung der Emissionen auf $\mathbf{E}_{2}$ nur eine Zukunftsschaden von $p_{2}{ }^{\infty}$ auftritt, wird zumindest ein Teil des Zukunftsschadens in Region 1 mit abgedeckt. Wird der Emissionsstandard für Region 2 so gesetzt, daß im Steady-State $\lambda_{2}^{2}{ }_{2}^{\infty}=$ $-\gamma_{2} p_{1}{ }^{\infty}$ gilt, wird dadurch, wie im Fall mit Zentralbehörde, der durch die Emissionstätigkeit in Region 2 verursachte Zukunftsschaden in Region 1 bei der Steuersetzung berücksichtigt.

Betrachten wir jetzt die Anpassungspfade der Region 2 an ein Steady-State und die Auswirkungen auf Region 1 [vgl. Schaubild 4.14].

(1.) Sei $S_{2}{ }^{0} \leq S_{2}$

Der stabile Ast der Trajektorie kann in diesem Fall nicht erreicht werden, da dann zu hohe Emissionen vorhanden wären, die den Emissionsstandard verletzen würden. Es ist in Region 2 deshalb optimal, den Emissionsstandard von Beginn an auszunutzen, so daß die Emissionen in Region 2 im Zeitablauf konstant bleiben. Für Region 1 bedeutet dies, daß jeweils ein konstanter Betrag an Emissionen von Region 2 importiert wird. Die Cournot-Annahme der Regionalregierung in Region 1 - die importierten Emissionen ändern sich im Zeitablauf nicht - erweist sich in diesem Fall als richtig. Wir haben dann im Prinzip die Anpassungen der Region 1 


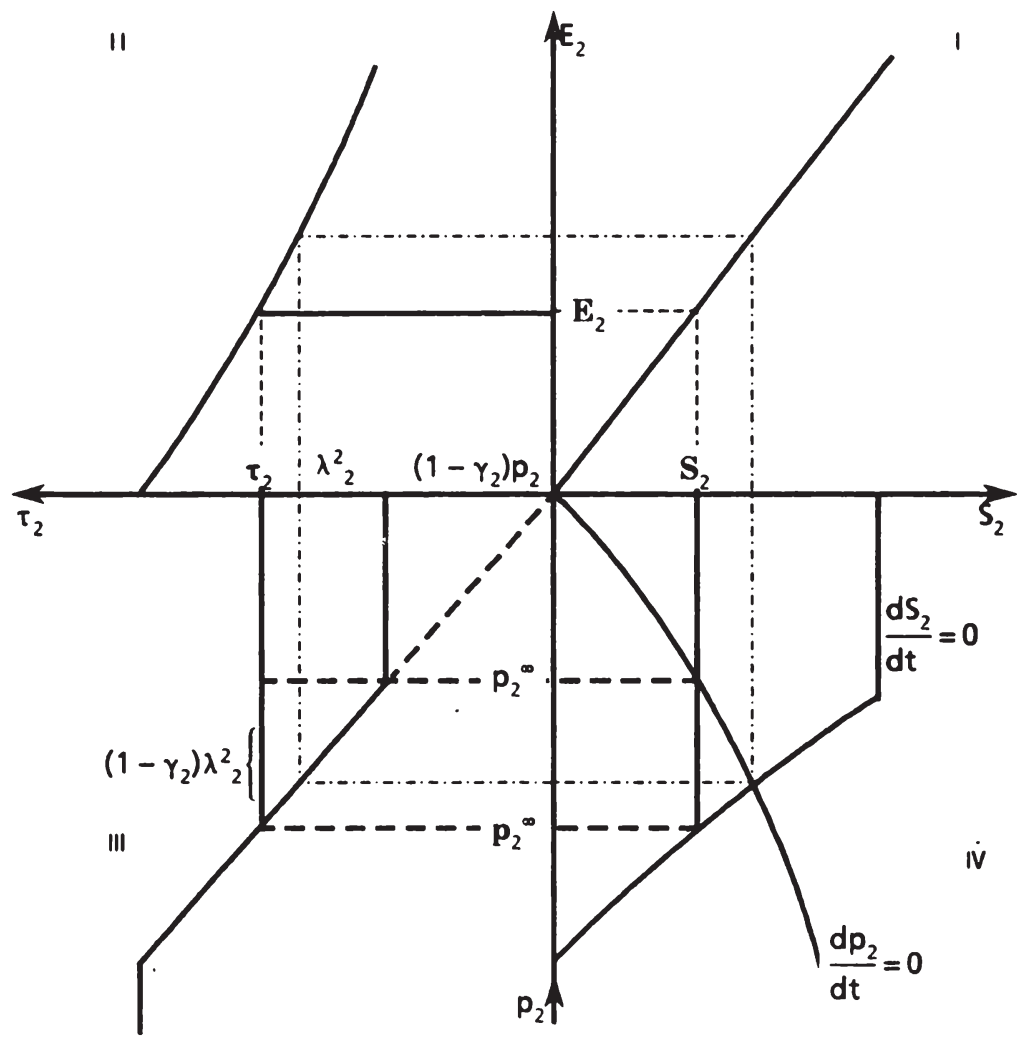

Schaubild 4.13: Emissionsstandard $\mathbf{E}_{2}$

wie im Grundmodell, wobei nur die $d S_{1} / d t=0$ Kurve weiter außen verläuft als ohne Diffusion [vgl. hierzu Kapitel 3, Abschnitt 2.1].

(2.) Sei $S_{2}{ }^{0}>S_{2}$

Für Region 2 bedeutet dies, da der Optimalwert der Immissionen kleiner als das unbeschränkte Optimum ist, daß weniger emittiert werden darf, um das beschränkte Optimum zu erreichen als das unbeschränkte.

Es können zwei Fälle unterschieden werden: Es gibt ein $\boldsymbol{S}_{2}$, für das 
die Beschränkung nicht mehr gilt, d. h. die Optimaltrajektorie fordert dann ein kleineres $E_{2}$ als die Beschränkung zuläßt. Dann gilt

$$
E_{2}<\mathbf{E}_{2}
$$

und damit $\lambda^{2}{ }_{2}=0$. Liegt $S_{2}{ }^{0}$ zwischen $S_{2}$ und $S_{2}$ greift die Restrikti-

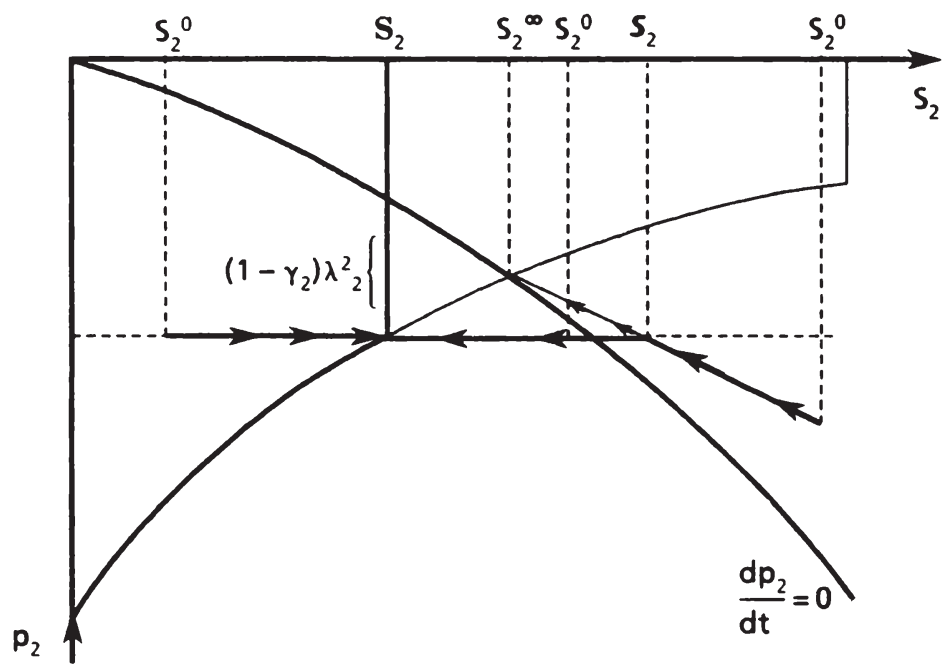

Schaubild 4.14: Anpassungspfade der Region 2 mit Standard

on von Anfang an und es gilt immer $E_{2}=\mathbf{E}_{2}$ und deshalb ist die optimale Steuer in Region 2 konstant:

$$
\tau_{2}=u^{2}{ }_{X}{ }^{2}{ }_{E} \mid E_{2}=\mathbf{E}_{2}=\text { konst. }
$$

und damit auch die Emissionen und die Güterproduktion, da

$$
X_{2}=f^{2}\left(\mathbf{R}_{2}, \mathbf{E}_{2}\right)
$$

Der Unterschied zwischen der optimalen Steuer $\tau_{2}$ und dem Zukunftsschaden $p_{2}$ wird durch $\lambda^{2}{ }_{2}$ ausgedrückt, dem Schattenpreis des Emissionsstandards. $\lambda^{2}{ }_{2}$ drückt den Wert des Konsumverzichtes aus, den es Region 2 kostet, die Emissionsrestriktion einzuhal- 
ten.

Für Region 1 heißt das wiederum, daß in jedem Zeitpunkt $t$ ein konstanter Betrag $\gamma_{2} \mathbf{E}_{2}$ an Emissionen importiert wird, so daß wieder auf das Grundmodell verwiesen werden kann (Kapitel 3, Abschnitt 2.3)

Ist andererseits $S_{2}{ }^{0}>S_{2}$, kann die Optimaltrajektorie in zwei Abschnitte aufgeteilt werden. So lange $S_{2}>S_{2}$ ist, befindet sich Region 2 auf der Optimaltrajektorie, wobei $\lambda_{2}^{2}=0$ ist. In $S_{2}=S_{2}$ ist $E_{2}$ $=E_{2}$. Gilt dann $S_{2}<S_{2}$ befinden wir uns wieder im ersten Fall. In $S_{2}=S_{2}$ hat die Trajektorie also einen Knick.

Zusammenfassend läßt sich sagen, daß eine Verstetigung der Politik für Region 1 erreicht wird, wenn eine Zentralregierung für Region 2 einen Emissionsstandard setzt.

\subsection{DIE AUSWIRKUNGEN VON PARAMETERÄNDERUNGEN}

In Tabelle 4.3 sind die Änderungen zusammengefaßt: Hier werden auch

\begin{tabular}{|c|c|c|c|c|c|c|c|c|c|c|}
\hline & $d E_{1}$ & $d S_{1}$ & $d p_{1}$ & $d \tau_{1}$ & $d X_{1}$ & $d S_{2}$ & $d p_{2}$ & $d \tau_{2}$ & $d X_{2}$ & $d \lambda^{2}{ }_{2}$ \\
\hline$d \delta$ & $\oplus$ & $\oplus$ & $\oplus$ & $\Theta$ & $\oplus$ & 0 & $\oplus$ & 0 & 0 & $\oplus$ \\
\hline$d \alpha_{2}$ & 0 & 0 & 0 & 0 & 0 & $\Theta$ & $\oplus$ & 0 & 0 & $\oplus$ \\
\hline$d \mathbf{R}_{2}$ & 0 & 0 & 0 & 0 & 0 & 0 & 0 & $\oplus$ & $\oplus$ & $\oplus$ \\
\hline$d \gamma_{2}$ & $\Theta$ & $\oplus$ & $\Theta$ & $\oplus$ & $\Theta$ & $\Theta$ & $\oplus$ & 0 & 0 & $\oplus$ \\
\hline$d \mathbf{E}_{2}$ & $\Theta$ & $\oplus$ & $\Theta$ & $\oplus$ & $\Theta$ & $\oplus$ & $\Theta$ & $\Theta$ & $\oplus$ & $\Theta$ \\
\hline
\end{tabular}

Tabelle 4.3: Auswirkungen von Parameteränderungen bei Standardsetzung in Region 2

die Auswirkungen auf die Variable $\lambda^{2}{ }_{2}$ der Region 2 betrachtet, die die Kosten des Konsumverzichtes bei Einhaltung des Emissionsstandards ausdrückt. 


\subsubsection{Die Auswirkungen auf Region 2}

Für den Emissionsstandard gilt: Je größer $\mathbf{E}_{2}$ ist, desto höher sind die Immissionen und die Bewertung des Zukunftsschadens sowie die Produktion, desto geringer ist die Steuer und desto niedriger sind die Kosten des Konsumverzichtes.

Die Größe des Assimilationskoeffizienten hat keinen Einfluß auf die Produktion und die Emissionssteuer, da der Emissionsstandard annahmegemäß weiterhin voll ausgenutzt werden soll. Jedoch sinken die Immissionen und die Bewertung des daraus resultierenden Schadens. Dieser Änderung in der Bewertung des Schadens steht eine im Betrag gleiche Erhöhung der Kosten des Konsumverzichtes gegenüber, da durch die größere Assimilationsfähigkeit die unbeschränkten Steady-State-Emissionen steigen könnten und damit mehr produziert werden könnte, und damit auch mehr konsumiert; diese Steigerung des Konsums ist jedoch nicht möglich, da nicht mehr als $\mathbf{E}_{2}$ Emissionen produziert werden dürfen.

Bei bindendem Emissionsstandard hat die Größe der Gegenwartsvorliebe in der Ökonomie auf die Immissionen, die Steuer und die Produktion in Region 2 keinen Einfluß. Nur die Bewertung des Schadens ändert sich der Schaden wird bei höherem $\delta$ geringer bewertet - und die Kosten des Konsumverzichtes steigen.

Die Höhe der Faktorausstattung hat auf die Immissionen und die Bewertung des Zukunftsschadens keinen Einfluß, da ja die Emissionen konstant sind. Da für einen höheren Ressourcenbestand die Grenzproduktivität der Emissionen steigt, steigt auch die Steuer und die Kosten des Konsumverzichts, da die Produktion zwar zunimmt, aber weniger stark als beim unbeschränkten Steady-State, weil die Emissionen konstant bleiben.

Zum Abschluß betrachten wir die Auswirkungen der Größe des Diffusionskoeffizienten auf die sich ergebende Optimallösung der Region 2. Je höher der Diffusionskoeffizient ist, desto niedriger sind die Immissionen und die daraus resultierenden Schäden. Die Steuer und die Produktion sind unabhängig von der Diffusion, sie bleiben auch bei höherem Diffusionskoeffizienten konstant. Durch die niedrigeren Immissionen wird der Unter- 
schied zwischen Steuer und Zukunftsschaden größer und damit werden die Kosten des Konsumverzichtes in Region 2 höher. Durch die Setzung eines bindenden Emissionsstandards durch die Zentralregierung ist für die emissionsexportierende Region ein Anreiz gegeben, den Diffusionskoeffizienten zu erhöhen, da dadurch der zeitpunktbezogene Nutzen in dieser Region steigt durch die Verminderung der Immissionen bei konstantem Konsum. Damit steigt die Gesamtwohlfahrt im Planungszeitraum.

\subsubsection{Die Auswirkungen auf Region 1}

Die Abhängigkeiten der autonomen Region 1 von ihrer eigenen Ressourcenausstattung und den Eigenschaften des Ökosystems $\left(\alpha_{1}\right)$ bleiben von der Emissionsstandardsetzung in Region 2 unberührt, da es keine Rückwirkungen von Region 2 gibt [vgl. Abschnitt 2.4.1]. Änderungen ergeben sich jedoch für die anderen Modellparameter.

Die Höhe der Gegenwartsvorliebe $\delta$ hat auf die Steady-State-Variablen äquivalente Auswirkungen wie in Kapitel 3, Abschnitt 2.2. Da sich der Emissionsimport aus Region 2 für eine höhere soziale Diskontrate nicht ändert, sondern ein fester Parameter ist, hat er keine Auswirkungen auf die Änderungsrichtung der Steady-State-Variablen in Region 1, jedoch auf deren Größe.

Die Assimilationskapazität des Ökosystems und die Faktorausstattung in Region 2 hat keine Rückwirkungen auf Region 1 bei bindendem Emissionsstandard, da sich die Emissionen aus Region 2 nicht ändern.

Die Größe des Diffusionskoeffizienten hat in der Richtung die gleichen Auswirkungen wie bei vollkommen autonomen Regionen: die Emissionen in Region 1 sinken, die Immissionen steigen, der Schattenpreis sinkt und die Steuer steigt, jedoch werden die Auswirkungen durch den bindenden Emissionsstandard abgemildert, wie ein Vergleich der Änderungsbedingungen zeigt. Zu diesem Zweck wird davon ausgegangen, daß $E_{2}{ }^{a}=\mathbf{E}_{2}$ ist, d. h. die Emissionen im Steady-State der Region 2 bei autonomer Optimierung sollen gerade so groß sein, wie der von der Zentralregierung vorgegebene Emissionsstandard. Jetzt wird in beiden Fällen der Diffusionskoeffizient erhöht: 


$$
\begin{aligned}
& \frac{d E_{1}^{a}}{d Y_{2}}=\frac{E_{\tau}^{1} u_{S S}^{1}\left(-Y_{2} E_{Y}^{2}-E_{2}^{a}\right)}{\Delta_{1}} \\
& \frac{d E_{1}^{s}}{d Y_{2}}=-\frac{E_{\tau}^{1} u_{S S}^{1} E_{2}}{\Delta_{1}}
\end{aligned}
$$

$\mathrm{Da} E_{2}{ }^{a}=\mathbf{E}_{2}$ gilt, ist

$$
\left|\frac{d E_{1}^{a}}{d Y_{2}}\right|>\left|\frac{d E_{1}^{s}}{d Y_{2}}\right|
$$

d. h. die Emissionen in Region 1 sinken bei autonomen Regionen stärker als wenn die Zentralregierung durch Standardsetzung in Region 2 eingreift. Für die anderen Variablen $\left(S_{1}, p_{1}, \tau_{1}, X_{1}\right)$ erhält man die analogen Ergebnisse.

Die Höhe des Emissionsstandards für Region 2 hat auf Region 1 die zu erwartenden Ergebnisse: Je höher $\mathbf{E}_{2}$ ist - je näher die Emissionen in Region 2 an dem unbeschränkten Optimalwert $E_{2}{ }^{a}$ sind -, desto niedriger sind die Emissionen in Region 1 und die Produktion, während die Immissionen, der Schaden und die Steuer umso höher sind.

Durch das Eingreifen der Zentralregierung durch die Setzung eines Emissionsstandards in der emissionsexportierenden Region wird die Abhängigkeit der emissionsimportierenden Region 1 von Region 2 abgeschwächt. Die emissionsimportierende Region ist weitgehend unabhängig von "Interna" der emissionsexportierenden Region $\left(\alpha_{2}, \mathbf{R}_{2}\right)$ und kann deshalb die Präferenzen ihrer Bewohner besser berücksichtigen unter weitestgehender Selbständigkeit. 


\section{ZUSAMMENFASSUNG}

In diesem Kapitel werden die Auswirkungen untersucht, die sich durch den Schadstofftransport von einer Region in die andere ergeben. Die untersuchte Ökonomie unterscheidet sich von der des Grundmodells in Kapitel 3 durch die Schadstoffdiffusion, die von Region 2, der emissionsexportierenden Region, nach Region 1, der emissionsimportierenden Region, führt. Diese Unterlieger-Oberlieger-Problematik wird unter drei unterschiedlichen institutionellen Arrangements betrachtet: Vollkommen autonome Regionen; Regionen, die von einer Zentralregierung gesteuert werden; für die emissionsexportierende Region wird von einer Zentralbehörde ein bindender Emissionsstandard gesetzt.

Sind die Regionen vollkommen autonom, dann muß die emissionsimportierende Region ihre Umweltpolitik nach der in der emissionsexportierenden Region betriebenen Umweltpolitik ausrichten. Die Umweltbehörde der emissionsexportierenden Region nimmt eine regionenegoistische Wohlfahrtsoptimierung vor und berücksichtigt die Schäden, die durch den Emissionsexport in der anderen Region entstehen, in ihrem Wohlfahrtskalkül nicht. Durch den Zusammenhang zwischen Region 1 und Region 2 sind folgende Eigenschaften des Steady-States der Ökonomie gegeben:

(i) Die Faktorausstattung und der Assimilationskoeffizient der Region 2 haben auf die Steady-State-Variablen der Region 1 Auswirkungen. Je höher die Faktorausstattung der Region 2 ist, desto mehr wird dort produziert - sowohl Konsumgüter als auch Emissionen -, so daß in Region 1 weniger produziert werden kann, während die Verschmutzung dort steigt.

(ii) Die in Kapitel 3 konstatierten Auswirkungen der sozialen Zeitpräferenzrate sind für Region 1 nicht länger gültig. Es kann mit Sicherheit nur gesagt werden, daß der Immissionsbestand der Region 1 umso größer ist, je höher die soziale Diskontrate ist.

(iii) Die durch den Emissionsexport hervorgerufene Abhängigkeit der Region 1 von Region 2 kommt durch den Diffusionskoeffizienten zum Ausdruck. Je höher der Diffusionskoeffizient ist, desto stärker sind die negativen Wohlfahrtseffekte auf Region 1. Eine Vergrößerung 
des Diffusionskoeffizienten (Bau höherer Schornsteine) in Region 2 hat auf die Wohlfahrt der Region 2 dann positive Auswirkungen, wenn in Region 2 "viel" emittiert wird. Es kann jedoch bei einer in der Ausgangslage strengen Umweltpolitik der Fall eintreten, daß durch eine Erhöhung des Diffusionskoeffizienten eine Wohlfahrtsminderung eintritt.

Diesem Modell autonomer Regionalbehörden wird ein Modell gegenübergestellt, bei dem eine Zentralbehörde die Wohlfahrt (gleichgewichteter) Regionen gemeinsam maximiert. Das sich ergebende Steady-State ist Pareto-optimal und es wird gezeigt, daß die gesamten Schäden, die durch Emissionen der emissionsexportierenden Region hervorgerufen werden, in dem Optimierungskalkül berücksichtigt werden. Es ergibt sich, daß in Region 1 mehr emittiert und damit produziert werden kann als im Fall autonomer Regionalbehörden, während in Region 2 die Emissionen und die Produktion niedriger sein müssen.

Durch die Berücksichtigung der Schäden in Region 1, die durch die Emissionen der Region 2 hervorgerufen werden, wird eine wechselseitige Abhängigkeit der Regionen definiert, so daß auch die "Interna" der Region 1 Auswirkungen auf die Steady-State-Variablen der Region 2 haben. Im einzelnen sind folgende Aussagen abgeleitet worden:

(i) Je höher die Faktorausstattung in einer Region ist, desto weniger darf in der anderen Region produziert werden.

(ii) Je höher der Assimilationskoeffizient in Region 1 ist, desto mehr wird in beiden Regionen produziert. Je höher der Assimilationskoeffizient in Region 2 ist, desto mehr wird in Region 2 und desto weniger wird in Region 1 produziert.

(iii) Die soziale Zeitpräferenzrate hat auf Region 2 weitgehend analoge Auswirkungen wie im autonomen Fall, nur in der Bewertung der Schäden und der Emissionstätigkeit ergibt sich kein eindeutiges Vorzeichen, da die Bewertung des Schadens in Region 1 mit berücksichtigt wird.

Für Region 1 bleiben die Aussagen des autonomen Falls erhalten. 
(iv) Für die Bewertung einer Politik des hohen Schornsteins in Region 2 sind die relativen Schäden der Regionen zueinander von Bedeutung. Es ist für bis auf die Diffusionsbeziehungen identische Regionen durchaus möglich, daß die Produktion in der emissionsexportierenden Region sinkt, während die Produktion in der emissionsimportierenden Region steigt. Definiert man die Analyse in Abschnitt 3 dieses Kapitels als kooperatives Vorgehen zwischen zwei autonomen Staaten, ist das erzielte Ergebnis dahingehend zu interpretieren, daß es für den emissionsexportierenden Staat nur dann Vorteile bringt, die Schornsteine zu erhöhen, wenn die Schäden in dem emissionsimportierenden Staat niedriger sind, die durch den Emissionsexport entstehen, als die in der emissionsexportierenden Region selbst.

Als dritter Ansatzpunkt der institutionellen Ausgestaltung der Ökonomie wird der Fall betrachtet, daß eine Zentralbehörde für die emissionsexportierende Region einen Emissionsstandard setzt, um so die Auswiirkungen durch den Emissionsexport auf die emissionsimportierende Region zu begrenzen. Die Emissionssteuer in der emissionsexportierenden Region 2 ist dann höher als die sozialen Kosten, die in Region 2 durch die Emissionstätigkeit entstehen, da dort Konsumverzicht geübt werden muß, um den Emissionsstandard einzuhalten. Dadurch kann, bei geeigneter Wahl des Standards durch die Zentralbehörde, ein äquivalentes Ergebnis zu der gemeinsamen Maximierung der Wohlfahrt beider Regionen erzielt werden.

Ist die emissionsexportierende Region 2 gezwungen, einen Emissionsstandard einzuhalten, wird die emissionsimportierende Region 1 weitgehend unabhängig von "Interna" der Region 2. Dies wird deutlich durch die fehlenden Auswirkungen des Faktorbestandes und der Assimilationsfähigkeit des Ökosystems der Region 2 auf die Steady-State-Variablen der Region 1. Darüberhinaus sind auch die Auswirkungen der Größe der sozialen Zeitpräferenzrate auf Region 1 die gleichen wie die im Grundmodell des Kapitels 3 abgeleiteten. Im einzelnen werden folgende Ergebnisse erzielt:

(i) Die soziale Zeitpräferenzrate hat keine Auswirkungen auf die Produktion und die Immissionen in Region 2. Es ändern sich nur die Bewertung der Schäden und die Kosten des Konsumverzichts. Für Region 1 bleiben die Aussagen des Grundmodells erhalten. 
(ii) Die Assimilationsfähigkeit des Ökosystems der Region 2 hat keine Auswirkungen auf Region 1. In Region 2 ändert sich für ein erhöhtes $\alpha_{2}$ die Produktion und die Emissionssteuer nicht, während die Immissionen sinken.

(iii) Auch die Höhe der Faktorausstattung der Region 2 hat nur Auswirkungen auf Region 2 selbst. Dort kann umso mehr produziert werden, je höher die Faktorausstattung ist.

(iv) Die Höhe des Emissionsstandards hat die zu erwartenden Auswirkungen auf beide Regionen.

(v) Wird der Diffusionskoeffizient erhöht, gelangen mehr Emissionen der Region 2 nach Region 1, so daß dort die Produktion sinkt während die Immissionen und die Emissionssteuer steigen. In Region 2 ändert sich an der Produktion nichts, auch die Emissionssteuer bleibt konstant, jedoch sinken die Immissionen. Durch die Setzung eines bindenden Emissionsstandards durch eine Zentralregierung ist deshalb für Region 2 ein Anreiz gegeben, eine Politik des hohen Schornsteins zu betreiben, da dadurch Wohlfahrtsgewinne erzielt werden können.

Für Region 1 ergibt sich also eine Verstetigung der Politik durch den bindenden Emissionsstandard in Region 2. Die Abhängigkeit der emissionsimportierenden Region von der emissionsexportierenden Region wird abgeschwächt; die Regionalregierung der Region 1 kann die Präferenzen ihrer Bewohner besser berücksichtigen. 


\section{ANHANG 4.I: Autonome Regionalregierungen}

\section{Die Änderungen für Region 2}

Folgendes Gleichungssystem wird total differenziert:

$$
\begin{aligned}
\left(1-\gamma_{2}\right) E_{2}-\alpha_{2} S_{2} & =0 \\
\left(\delta+\alpha_{2}\right) p_{2}-u^{2}{ }_{S} & =0 \\
\tau_{2}+\left(1-\gamma_{2}\right) p_{2} & =0 \\
E_{2}-E^{2}\left(\tau_{2}\right) & =0
\end{aligned}
$$

Man erhält

$$
\begin{aligned}
& {\left[\begin{array}{cccc}
1-\gamma_{2} & -\alpha_{2} & 0 & 0 \\
0 & -u_{S S}^{2} & \delta+\alpha_{2} & 0 \\
0 & 0 & 1-\gamma_{2} & 1 \\
1 & 0 & 0 & -E^{2}{ }_{\tau}
\end{array}\right]\left[\begin{array}{c}
d E_{2} \\
d S_{2} \\
d p_{2} \\
d \tau_{2}
\end{array}\right]=\left[\begin{array}{l}
E_{2} \\
0 \\
p_{2} \\
0
\end{array}\right] d \gamma_{2}+\left[\begin{array}{c}
0 \\
-p_{2} \\
0 \\
0
\end{array}\right] d \delta+} \\
& +\left[\begin{array}{c}
S_{2} \\
-p_{2} \\
0 \\
0
\end{array}\right] d \alpha_{2}+\left[\begin{array}{c}
0 \\
0 \\
0 \\
E_{R}^{2}
\end{array}\right] d \mathbf{R}_{2}
\end{aligned}
$$

Die Systemdeterminante ist

$$
\Delta_{2}=\left(1-\gamma_{2}\right)^{2} u_{S S}^{2} E^{2}{ }_{\tau}+\alpha_{2}\left(\delta+\alpha_{2}\right)>0
$$




\subsection{Die Änderungsrichtungen für ein erhöhtes $\gamma_{2}$ :}

$$
\begin{aligned}
& \frac{d E_{2}}{d Y_{2}}=\frac{E_{\tau}^{2}\left[\left(1-Y_{2}\right) u_{S S}^{2} E_{2}+a_{2}\left(\delta+a_{2}\right) p_{2} \mid\right.}{\Delta_{2}}>0 \\
& \frac{d S_{2}}{d Y_{2}}=\frac{\left(\delta+a_{2}\right)\left|\left(1-Y_{2}\right) E_{\tau}^{2} p_{2}-E_{2}\right|}{\Delta_{2}} \geq_{0} \\
& \frac{d p_{2}}{d Y_{2}}=\frac{u_{S S}^{2}\left[\left(1-Y_{2}\right) E_{\tau}^{2} p_{2}-E_{2} \mid\right.}{\Delta_{2}} \geq_{0} \\
& \frac{d \tau_{2}}{d Y_{2}}=\frac{\left(1-Y_{2}\right) u_{S S}^{2} E_{2}+a_{2}\left(\delta+a_{2}\right) p_{2}}{\Delta_{2}}<0
\end{aligned}
$$

1.2 Die Änderungen für ein erhöhtes $\delta$ :

$$
\begin{aligned}
& \frac{d E_{2}}{d \delta}=\frac{\left(1-\mathrm{Y}_{2}\right) \mathrm{a}_{2} E_{\tau}^{2} p_{2}}{\Delta_{2}}>0 \\
& \frac{d S_{2}}{d \delta}=\frac{\left(1-\mathrm{Y}_{2}\right)^{2} E_{\tau}^{2} p_{2}}{\Delta_{2}}>0 \\
& \frac{d p_{2}}{d \delta}=\frac{-\mathrm{a}_{2} p_{2}}{\Delta_{2}}>0 \\
& \frac{d \tau_{2}}{d \delta}=\frac{\left(1-\mathrm{Y}_{2}\right) \mathrm{a}_{2} p_{2}}{\Delta_{2}}<0
\end{aligned}
$$


1.3 Die Änderungen für ein erhöhtes $\alpha_{2}$ :

$$
\begin{aligned}
& \frac{d E_{2}}{d a_{2}}=\frac{\left(1-\mathrm{Y}_{2}\right) E_{\tau}^{2}\left[u_{S S}^{2} S_{2}+a_{2} p_{2}\right]}{\Delta_{2}}>0 \\
& \frac{d S_{2}}{d a_{2}}=\frac{\left(1-\mathrm{Y}_{2}\right)^{2} E_{\tau}^{2} p_{2}-\left(\delta+a_{2}\right) S_{2}}{\Delta_{2}} \geq 0 \\
& \frac{d p_{2}}{d a_{2}}=\frac{-\left[u_{S S}^{2} S_{2}+a_{2} p_{2}\right]}{\Delta_{2}}>0 \\
& \frac{d \tau_{2}}{d a_{2}}=\frac{\left(1-Y_{2}\right)\left[u_{S S}^{2} S_{2}+a_{2} p_{2}\right]}{\Delta_{2}}<0
\end{aligned}
$$

1.4 Die Änderungen für ein erhöhtes $R_{2}$ :

$$
\begin{aligned}
& \frac{d E_{2}}{d \mathbf{R}_{2}}=\frac{a_{2}\left(\delta+a_{2}\right) E_{R}^{2}}{\Delta_{2}}>0 \\
& \frac{d S_{2}}{d \mathbf{R}_{2}}=\frac{\left(1-y_{2}\right)\left(\delta+a_{2}\right) E_{R}^{2}}{\Delta_{2}}>0 \\
& \frac{d p_{2}}{d R_{2}}=\frac{\left(1-Y_{2}\right) u_{S S}^{2} E_{R}^{2}}{\Delta_{2}}<0 \\
& \frac{d \tau_{2}}{d R_{2}}=\frac{-\left(1-\gamma_{2}\right)^{2} u_{S S}^{2} E_{R}^{2}}{\Delta_{2}}>0
\end{aligned}
$$




\section{Die Änderungen für Region 1}

Für Region 1 erhalten wir ein analoges Gleichungssystem, bei dem allerdings die Abhängigkeit von Region 2 mit berücksichtigt werden muß:

$$
\begin{aligned}
E_{1}-\alpha_{1} S_{1} & =-\gamma_{2} E_{2} \\
\left(\delta+\alpha_{1}\right) p_{1}-u_{S}^{1} & =0 \\
\tau_{1}+p_{1} & =0 \\
E_{1}-E^{1}\left(\tau_{1}\right) & =0
\end{aligned}
$$

Totale Differentation ergibt

$$
\left[\begin{array}{cccc}
1 & -\alpha_{1} & 0 & 0 \\
0 & -u_{S S}^{1} & \delta+\alpha_{1} & 0 \\
0 & 0 & 1 & 1 \\
1 & 0 & 0 & -E_{\tau}^{1} \tau
\end{array}\right]\left[\begin{array}{l}
d E_{1} \\
d S_{1} \\
d p_{1} \\
d \tau_{1}
\end{array}\right]=\left[\begin{array}{l}
\mathbf{e}_{2} \\
0 \\
0 \\
0
\end{array}\right] d \gamma_{2}+\left[\begin{array}{c}
\gamma_{2} E^{2} \delta \\
-p_{1} \\
0 \\
0
\end{array}\right] d \delta+
$$$$
+\left[\begin{array}{c}
S_{1} \\
-p_{1} \\
0 \\
0
\end{array}\right] d \alpha_{1}+\left[\begin{array}{c}
0 \\
0 \\
0 \\
E^{1}
\end{array}\right] d \mathbf{R}_{1}+\left[\begin{array}{c}
\gamma_{2} E_{z}^{2} \\
0 \\
0 \\
0
\end{array}\right] d z
$$

mit

$$
\begin{aligned}
& e_{2}=-\gamma_{2} E^{2}{ }_{\gamma}-E_{2}<0, \\
& z=\alpha_{2}, \mathbf{R}_{2}
\end{aligned}
$$


Die Systemdeterminante ist

$$
\Delta_{1}=u_{S S^{1}}^{E^{1}}{ }_{\tau}+\alpha_{1}\left(\delta+\alpha_{1}\right)>0
$$

2.1 Die Änderungen für ein größeres $\gamma_{2}$ :

$$
\begin{aligned}
& \frac{d E_{1}}{d \mathrm{Y}_{2}}=\frac{E_{\tau}^{1} u_{S S}^{1} \mathbf{e}_{2}}{\Delta_{1}}<0 \\
& \frac{d S_{1}}{d \mathrm{Y}_{2}}=\frac{-\left(\delta+\mathrm{a}_{1}\right) \mathbf{e}_{2}}{\Delta_{1}}>0 \\
& \frac{d p_{1}}{d \mathrm{Y}_{2}}=\frac{-u_{S S}^{1} \mathbf{e}_{2}}{\Delta_{1}}<0 \\
& \frac{d \tau_{1}}{d \mathrm{Y}_{2}}=\frac{u_{S S^{2}}^{1} \mathrm{e}_{2}}{\Delta_{1}}>0
\end{aligned}
$$

2.2 Die Änderungen für ein erhöhtes $\delta$ :

$$
\begin{aligned}
& \frac{d E_{1}}{d \delta}=\frac{E_{\imath}^{1}\left[a_{1} p_{1}-Y_{2} E_{\delta}^{2} u_{S S}^{1}\right]}{\Delta_{1}} \geq_{<0} \\
& \frac{d S_{1}}{d \delta}=\frac{\left.E_{\imath}^{1} p_{1}+Y_{2}\left(\delta+a_{1}\right) E_{\delta}^{2}\right]}{\Delta_{1}}>0 \\
& \frac{d p_{1}}{d \delta}=\frac{Y_{2} E_{8}^{2} u_{S S}^{1}-a_{1} p_{1}}{\Delta_{1}} \geq_{0}
\end{aligned}
$$




$$
\frac{d \tau_{1}}{d \delta}=\frac{a_{1} p_{1}-\chi_{2} E_{\delta}^{2} u_{S S}^{1}}{\Delta_{1}} \geq_{0}
$$

2.3 Die Änderungen für ein erhöhtes $\alpha_{1}$ :

$$
\begin{aligned}
& \frac{d E_{1}}{d a_{1}}=\frac{E_{c}^{1}\left[u_{S S}^{1} S_{1}+a_{1} p_{1}\right]}{\Delta_{1}}>0 \\
& \frac{d S_{1}}{d a_{1}}=\frac{E_{t}^{1} p_{1}-\left(\delta+a_{1}\right) S_{1}}{\Delta_{1}} \geq_{0} \\
& \frac{d p_{1}}{d a_{1}}=\frac{-\left[u_{S S}^{1} S_{1}+a_{1} p_{1}\right]}{\Delta_{1}}>0 \\
& \frac{d \tau_{1}}{d a_{1}}=\frac{u_{S S_{1}}^{1} S_{1}+a_{1} p_{1}}{\Delta_{1}}<0
\end{aligned}
$$

2.4 Die Änderungen für ein erhöhtes $\mathbf{R}_{1}$ :

$$
\begin{aligned}
& \frac{d E_{1}}{d \mathbf{R}_{1}}=\frac{a_{1}\left(\delta+a_{1}\right) E_{R}^{1}}{\Delta_{1}}>0 \\
& \frac{d S_{1}}{d \mathbf{R}_{1}}=\frac{\left(\delta+a_{1}\right) E_{R}^{1}}{\Delta_{1}}>0 \\
& \frac{d p_{1}}{d R_{1}}=\frac{u_{S S}^{1} E_{R}^{1}}{\Delta_{1}}<0
\end{aligned}
$$




$$
\frac{d \tau_{1}}{d \mathbf{R}_{1}}=-\frac{u_{S S}^{1} E_{\mathbf{R}}^{1}}{\Delta_{1}}>0
$$

2.5 Die Änderungen in Region 1 bei erhöhtem $\alpha_{2}$ und $R_{2}$ :

Es gilt $\quad E_{\alpha}^{2}=d E_{2} / d \alpha_{2}>0$

und $\quad E^{2}{ }_{\mathbf{R}}=d E_{2} / d \mathbf{R}_{2}>0$

und damit $E_{z}^{2}>0\left(z=\alpha_{2}, \mathbf{R}_{2}\right)$

$$
\begin{aligned}
& \frac{d E_{1}}{d z}=\frac{-Y_{2} E_{\tau}^{1} u_{S S}^{1} E_{z}^{2}}{\Delta_{1}}<0 \\
& \frac{d S_{1}}{d z}=\frac{Y_{2}\left(\delta+a_{1}\right) E_{2}^{2}}{\Delta_{1}}>0 \\
& \frac{d p_{1}}{d z}=\frac{Y_{2} u_{S S}^{1} E_{2}^{2}}{\Delta_{1}}<0 \\
& \frac{d \tau_{1}}{d z}=-\frac{Y_{2} u_{S S}^{1} E_{z}^{2}}{\Delta_{1}}>0
\end{aligned}
$$




\section{Anhang 4.II: Zentralregierung}

Für den Zentralregierungsfall können nicht mehr zwei unabhängige Systeme betrachtet werden, sondern es ist ein interdependentes System zu analysieren:

$$
\begin{aligned}
E_{1}-\alpha_{1} S_{1}+\gamma_{2} E_{2} & =0 \\
\left(\delta+\alpha_{1}\right) p_{1}-u^{1}{ }_{S} & =0 \\
\tau_{1}+p_{1} & =0 \\
E_{1}-E^{1}\left(\tau_{1}\right) & =0 \\
\left(1-\gamma_{2}\right) E_{2}-\alpha_{2} S_{2} & =0 \\
\left(\delta+\alpha_{2}\right) p_{2}-u^{2}{ }_{S} & =0 \\
\tau_{2}+\left(1-\gamma_{2}\right) p_{2}+\gamma_{2} p_{1} & =0 \\
E_{2}-E^{2}\left(\tau_{2}\right) & =0
\end{aligned}
$$

Totale Differentiation ergibt

$\left[\begin{array}{cccccccc}1 & -\alpha_{1} & 0 & 0 & \gamma_{2} & 0 & 0 & 0 \\ 0 & -u_{S S}^{1} & \delta+\alpha_{1} & 0 & 0 & 0 & 0 & 0 \\ 0 & 0 & 1 & 1 & 0 & 0 & 0 & 0 \\ 1 & 0 & 0 & -E^{1} \tau & 0 & 0 & 0 & 0 \\ 0 & 0 & 0 & 0 & 1-\gamma_{2} & -\alpha_{2} & 0 & 0 \\ 0 & 0 & 0 & 0 & 0 & -u^{2} S_{S} & \delta+\alpha_{2} & 0 \\ 0 & 0 & \gamma_{2} & 0 & 0 & 0 & 1-\gamma_{2} & 1 \\ 0 & 0 & 0 & 0 & 1 & 0 & 0 & -E_{\tau}^{2}\end{array}\right]\left[\begin{array}{l}d E_{1} \\ d S_{1} \\ d p_{1} \\ d \tau_{1} \\ d E_{2} \\ d S_{2} \\ d p_{2} \\ d \tau_{2}\end{array}\right]=$




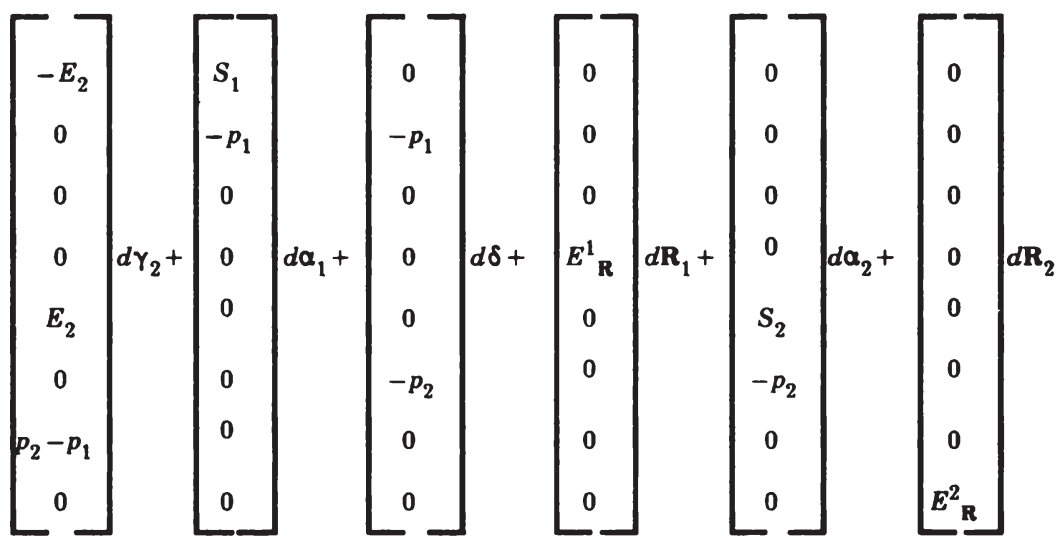

Die Systemdeterminante ist

$$
\begin{aligned}
\Delta= & {\left[u_{S S}^{1} E^{1}{ }_{\tau}+\alpha_{1}\left(\delta+\alpha_{1}\right)\right]\left[\left(1-\gamma_{2}\right)^{2} u_{S S}^{2} E^{2}{ }_{\tau}+\alpha_{2}\left(\delta+\alpha_{2}\right)\right]+} \\
& \gamma_{2}^{2} \alpha_{2}\left(\delta+\alpha_{2}\right) u_{S S}^{1} E_{\tau}^{2}>0
\end{aligned}
$$

1. Die Änderungen für ein erhöhtes $\alpha_{1}$ :

$$
\begin{aligned}
& \frac{d E_{1}}{d a_{1}}=\frac{E_{i}^{1}\left[u_{S S}^{1} S_{1}+a_{1} p_{1}\right]\left[\left(1-Y_{2}\right)^{2} u_{S S}^{2} E_{\tau}^{2}+a_{2}\left(\delta+a_{2}\right)\right]}{\Delta}>0 \\
& \frac{d S_{1}}{d a_{1}}=\frac{\left[p_{1} E_{\tau}^{1}-\left(\delta+a_{1}\right) S_{1}\right]\left[\left(1-Y_{2}\right)^{2} u_{S S}^{2} E_{\tau}^{2}+a_{2}\left(\delta+a_{2}\right)\right]+a_{2}\left(\delta+a_{2}\right) Y_{2}^{2} E_{\tau}^{2} p_{1}}{\Delta} \geq 0 \\
& \frac{d p_{1}}{d a_{1}}=\frac{-\left[u_{S S}^{1} S_{1}+a_{1} p_{1}\right]\left[\left(1-Y_{2}\right)^{2} u_{S S}^{2} E_{\tau}^{2}+a_{2}\left(\delta+a_{2}\right)\right]}{\Delta}>0 \\
& \frac{d \tau_{1}}{d a_{1}}=\frac{\left[u_{S S}^{1} S_{1}+a_{1} p_{1}\right]\left[\left(1-Y_{2}\right)^{2} u_{S S}^{2} E_{\tau}^{2}+a_{2}\left(\delta+a_{2}\right)\right]}{\Delta}<0
\end{aligned}
$$


$\frac{d E_{2}}{d \mathrm{a}_{1}}=\frac{\mathrm{a}_{2}\left(\delta+\mathrm{a}_{2}\right) \mathrm{Y}_{2} E_{\mathrm{t}}^{2}\left[u_{S S}^{1} S_{1}+a_{1} p_{1}\right]}{\Delta}>0$

$\frac{d S_{2}}{d a_{1}}=\frac{\left(\delta+a_{2}\right)\left(1-\mathrm{Y}_{2}\right) \mathrm{Y}_{2} E_{\mathrm{r}}^{2}\left[u_{S S}^{1} S_{1}+\mathrm{a}_{1} p_{1}\right]}{\Delta}>0$

$\frac{d p_{2}}{d a_{1}}=\frac{\left(1-\mathrm{Y}_{2}\right) \mathrm{Y}_{2} E_{\tau}^{2} u_{S S}^{2}\left[u_{S S}^{1} S_{1}+a_{1} p_{1}\right]}{\Delta}<0$

$\frac{d \tau_{2}}{d a_{1}}=\frac{a_{2}\left(\delta+a_{2}\right) y_{2}\left[u_{S S}^{1} S_{1}+a_{1} p_{1}\right]}{\Delta}<0$

2. Die Änderungen für ein erhöhtes $\alpha_{2}$ :

$$
\begin{aligned}
& \frac{d E_{1}}{d \mathrm{a}_{2}}=\frac{-Y_{2}\left(1-Y_{2}\right) u_{S S}^{1} E_{\mathrm{r}}^{1} E_{\tau}^{2}\left[u_{S S}^{2} S_{2}+\mathrm{a}_{2} p_{2}\right]}{\Delta}<0 \\
& \frac{d S_{1}}{d a_{2}}=\frac{\left(\delta+a_{1}\right) Y_{2}\left(1-y_{2}\right) E_{\tau}^{2}\left[u_{S S}^{2} S_{2}+a_{2} p_{2}\right]}{\Delta}>0
\end{aligned}
$$

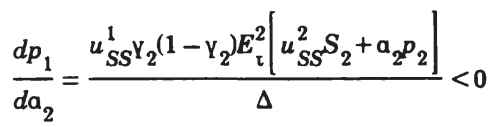

$$
\begin{aligned}
& \frac{d \tau_{1}}{d a_{2}}=-\frac{u_{S S Y_{2}}^{1}\left(1-Y_{2}\right) E_{\tau}^{2}\left[u_{S S}^{2} S_{2}+a_{2} p_{2}\right]}{\Delta}>0
\end{aligned}
$$




$$
\begin{aligned}
& \frac{d E_{2}}{d a_{2}}=\frac{\left(1-Y_{2}\right) E_{\tau}^{2}\left[u_{S S}^{1} E_{\tau}^{1}+a_{1}\left(\delta+a_{1}\right)\right]\left[u_{S S}^{2} S_{2}+a_{2} p_{2}\right]}{\Delta}>0 \\
& \frac{d S_{2}}{d a_{2}}=\frac{-\left(\delta+a_{2}\right) Y_{2}^{2} u_{S S}^{1} E_{\tau}^{2} S_{2}+\left\lfloor\left(1-Y_{2}\right)^{2} E_{\tau}^{2} p_{2}-\left(\delta+a_{2}\right) S_{2}\right]\left|u_{S S}^{1} E_{\tau}^{1}+a_{1}\left(\delta+a_{1}\right)\right|}{\Delta} \geq 0 \\
& \frac{d p_{2}}{d a_{2}}=\frac{-\left[u_{S S}^{1}\left(E_{\tau}^{1}+Y_{2}^{2} E_{\tau}^{2}\right)+a_{1}\left(\delta+a_{1}\right)|| u_{S S}^{2} S_{2}+a_{2} p_{2}\right]}{\Delta}>0 \\
& \frac{d \tau_{2}}{d a_{2}}=\frac{\left(1-Y_{2}\right)\left[u_{S S}^{1} E_{\tau}^{1}+a_{1}\left(\delta+a_{1}\right)\right]\left[u_{S S}^{2} S_{2}+a_{2} p_{2}\right\rfloor}{\Delta}<0
\end{aligned}
$$

\section{Die Änderungen für ein erhöhtes $\delta$ :}

$$
\begin{aligned}
& \frac{d E_{1}}{d \delta}=\frac{E_{\tau}^{1}\left\{a_{1} p_{1}\left[\left(1-Y_{2}\right)^{2} u_{S S}^{2} E_{\tau}^{2}+a_{2}\left(\delta+a_{2}\right)\right]-a_{2} p_{2} Y_{2}\left(1-Y_{2}\right) u_{S S}^{1} E_{\tau}^{2}\right\}}{\Delta} \geq_{<} \\
& \frac{d S_{1}}{d \delta}=\frac{E_{\tau}^{1} p_{1}\left[\left(1-Y_{2}\right)^{2} u_{S S}^{2} E_{\tau}^{2}+a_{2}\left(\delta+a_{2}\right)\right]+a_{2} Y_{2} E_{\tau}^{2}\left(Y_{2}\left(\delta+a_{2}\right) p_{1}+\left(1-Y_{2}\right)\left(\delta+a_{1}\right) p_{2}\right)}{\Delta}>0
\end{aligned}
$$

$$
\frac{d p_{1}}{d \delta}=-\frac{a_{1} p_{1}\left[\left(1-Y_{2}\right)^{2} u_{S S}^{2} E_{\tau}^{2}+a_{2}\left(\delta+a_{2}\right)\right]-a_{2} p_{2} Y_{2}\left(1-Y_{2}\right) u_{S S}^{1} E_{\tau}^{2}}{\Delta} \geq 0
$$

$$
\frac{d \tau_{1}}{d \delta}=\frac{a_{1} p_{1}\left[\left(1-Y_{2}\right)^{2} u_{S S}^{2} E_{\tau}^{2}+a_{2}\left(\delta+a_{2}\right)\right]-a_{2} p_{2} Y_{2}\left(1-Y_{2}\right) u_{S S}^{1} E_{\tau}^{2}}{\Delta} \geq 0
$$


$\frac{d E_{2}}{d \delta}=\frac{a_{2} E_{\tau}^{2}\left\{Y_{2} a_{1}\left(\delta+a_{2}\right) p_{1}+\left(1-Y_{2}\right) p_{2}\left[u_{S S}^{1} E_{\tau}^{1}+a_{1}\left(\delta+a_{1}\right)\right]\right\}}{\Delta}>0$

$\frac{d S_{2}}{d \delta}=\frac{Y_{2}\left(1-Y_{2}\right) a_{1}\left(\delta+a_{2}\right) p_{1} E_{\tau}^{2}+\left(1-Y_{2}\right)^{2} p_{2} E_{\tau}^{2}\left[u_{S S}^{1} E_{\tau}^{1}+a_{1}\left(\delta+a_{1}\right)\right]}{\Delta}>0$

$\frac{d p_{2}}{d \delta}=\frac{Y_{2}\left(1-Y_{2}\right) a_{1} u_{S S}^{2} p_{1} E_{\tau}^{2}-a_{2} p_{2}\left[Y_{2}^{2} u_{S S}^{1} E_{\tau}^{2}+u_{S S}^{1} E_{\tau}^{1}+a_{1}\left(\delta+a_{1}\right)\right]}{\Delta} \geq 0$

$\frac{d \tau_{2}}{d \delta}=\frac{a_{2}\left\{Y_{2} a_{1}\left(\delta+a_{2}\right) p_{1}+\left(1-Y_{2}\right) p_{2}\left[u_{S S}^{1} E_{\tau}^{1}+a_{1}\left(\delta+a_{1}\right)\right]\right\}}{\Delta}<0$

4. Die Änderungen für ein erhöhtes $\mathbf{R}_{1}$ :

$$
\begin{aligned}
& \frac{d E_{1}}{d \mathbf{R}_{1}}=\frac{E_{\mathbf{R}}^{1}\left\{a_{1}\left(\delta+a_{1}\right)\left[\left(1-Y_{2}\right)^{2} u_{S S}^{2} E_{\tau}^{2}+a_{2}\left(\delta+a_{2}\right)\right]+a_{2}\left(\delta+a_{2}\right) Y_{2}^{2} u_{S S}^{1} E_{\tau}^{2}\right\}}{\Delta}>0 \\
& \frac{d S_{1}}{d R_{1}}=\frac{E_{R}^{1}\left(\delta+a_{1}\right)\left[\left(1-Y_{2}\right)^{2} u_{S S}^{2} E_{\tau}^{2}+a_{2}\left(\delta+a_{2}\right)\right]}{\Delta}>0 \\
& \frac{d p_{1}}{d R_{1}}=\frac{u_{S S}^{1} E_{R}^{1}\left[\left(1-Y_{2}\right)^{2} u_{S S}^{2} E_{\tau}^{2}+a_{2}\left(\delta+a_{2}\right)\right]}{\Delta}<0 \\
& \frac{d \tau_{1}}{d R_{1}}=-\frac{u_{S S}^{1} E_{R}^{1}\left[\left(1-Y_{2}\right)^{2} u_{S S}^{2} E_{\tau}^{2}+a_{2}\left(\delta+a_{2}\right)\right]}{\Delta}>0
\end{aligned}
$$




$$
\begin{aligned}
& \frac{d E_{2}}{d \mathbf{R}_{1}}=-\frac{E_{\mathbf{R}}^{1} \mathrm{a}_{2}\left(\delta+\mathrm{a}_{2}\right) \mathrm{Y}_{2} u_{S S}^{1} E_{\mathrm{\tau}}^{2}}{\Delta}<0 \\
& \frac{d S_{2}}{d \mathbf{R}_{1}}=-\frac{E_{\mathbf{R}^{1}\left(\delta+\mathrm{a}_{2}\right) \mathrm{Y}_{2}\left(1-\mathrm{Y}_{2}\right) u_{S S}^{1} E_{\mathrm{\tau}}^{2}}}{\Delta}<0 \\
& \frac{d p_{2}}{d \mathbf{R}_{1}}=-\frac{E_{\mathbf{R}_{2} \mathrm{Y}_{2}\left(1-\mathrm{Y}_{2}\right) u_{S S}^{1} u_{S S}^{2} E_{\mathrm{\tau}}^{2}}^{\Delta}>0}{\Delta}>0 \\
& \frac{d \tau_{2}}{d \mathbf{R}_{1}}=-\frac{E_{\mathbf{R}^{\mathrm{a}}}^{1} \mathrm{a}_{2}\left(\delta+\mathrm{a}_{2}\right) \mathrm{Y}_{2} u_{S S}^{1}}{\Delta}>0
\end{aligned}
$$

5. Die Änderungen für ein erhöhtes $\mathbf{R}_{2}$ :

$$
\begin{aligned}
& \frac{d E_{1}}{d \mathbf{R}_{2}}=-\frac{E_{\mathbf{R}_{2}}^{2} \mathrm{a}_{2}\left(\delta+\mathrm{a}_{2}\right) \mathrm{Y}_{2} u_{S S}^{1} E_{\mathrm{\tau}}^{1}}{\Delta}<0 \\
& \frac{d S_{1}}{d \mathbf{R}_{2}}=\frac{E_{\mathrm{R}}^{2}\left(\delta+a_{1}\right) a_{2}\left(\delta+a_{2}\right) \mathrm{Y}_{2}}{\Delta}>0 \\
& \frac{d p_{1}}{d \mathbf{R}_{2}}=\frac{E_{R^{2}}^{2}{ }_{S S}^{1} a_{2}\left(\delta+a_{2}\right) Y_{2}}{\Delta}<0 \\
& \frac{d \tau_{1}}{d \mathbf{R}_{2}}=-\frac{E_{R^{2} u_{S S}^{1} a_{2}\left(\delta+a_{2}\right) Y_{2}}}{\Delta}>0 \\
& \frac{d E_{2}}{d R_{2}}=\frac{E_{R_{2}}^{2} a_{2}\left(\delta+a_{2}\right)\left[u_{S S}^{1} E_{t}^{1}+a_{1}\left(\delta+a_{1}\right)\right]}{\Delta}>0
\end{aligned}
$$




$$
\begin{aligned}
& \frac{d S_{2}}{d \mathbf{R}_{2}}=\frac{E_{\mathbf{R}}^{2}\left(1-Y_{2}\right)\left(\delta+a_{2}\right)\left[u_{S S}^{1} E_{\tau}^{1}+a_{1}\left(\delta+a_{1}\right) \mid\right.}{\Delta}>0 \\
& \frac{d p_{2}}{d \mathbf{R}_{2}}=\frac{E_{\mathbf{R}}^{2}\left(1-Y_{2}\right) u_{S S}^{2}\left[u_{S S}^{1} E_{\tau}^{1}+a_{1}\left(\delta+a_{1}\right)\right]}{\Delta}<0 \\
& \frac{d \tau_{2}}{d \mathbf{R}_{2}}=-\frac{E_{\mathbf{R}}^{2}\left\{\left(1-Y_{2}\right)^{2} u_{S S}^{2}\left[u_{S S^{2}}^{1} E_{\tau}^{1}+a_{1}\left(\delta+a_{1}\right)\right]+Y_{2}^{2} a_{2}\left(\delta+a_{2}\right) u_{S S}^{1}\right\}}{\Delta}>0
\end{aligned}
$$

\section{Die Änderungen für ein erhöhtes $\gamma_{2}$ :}

Zur Analyse einer Änderung des Diffusionskoeffizienten müssen die Regionen näher spezifiziert werden, da das Verhältnis der Schattenpreise in beiden Regionen zueinander die Änderung der Steady-State-Werte bestimmt.

Es wird davon ausgegangen, daß die Präferenzen - und damit die Nutzenfunktionen - und die Produktionstechnologien in beiden Regionen identisch sind. Weiterhin wird unterstellt, daß auch die Ökosysteme in beiden Regionen bezüglich des Assimilationskoeffizienten identisch sind, so daß sich die Regionen nur durch die Diffusion unterscheiden. Es ist jetzt zu untersuchen, in welchem Verhältnis die Schattenpreise zueinander stehen.

Sei

$$
\begin{aligned}
& p_{1} \geq p_{2} \\
& \Rightarrow p_{1}=\left(u^{1} s^{j}(\delta+\alpha)\right) \geq\left(u^{2} S^{J}(\delta+\alpha)\right)=p_{2} \\
& \Rightarrow u^{1} S^{\prime 2}{ }_{S} \leq 1 \Rightarrow S_{1} \leq S_{2} \\
& \Rightarrow \alpha\left(S_{1}-S_{2}\right)=E_{1}-\left(1-2 \gamma_{2}\right) E_{2} \leq 0^{15)} \Rightarrow E_{1}<E_{2} \\
& \Rightarrow \tau_{1}>\tau_{2}
\end{aligned}
$$

15) Ist $\gamma_{2} \geq \frac{1}{2}$, dann tritt hier schon ein Widerspruch auf, da dann $E_{1}-\left(1-2 \gamma_{2}\right) E_{2}>0$ ist und damit $S_{1} \leq S_{2}$ nicht gelten kann. 


$$
\begin{aligned}
& \Rightarrow \tau_{2}+\left(1-\gamma_{2}\right) p_{2}-\gamma_{2} \tau_{1}=0 \Rightarrow \tau_{2}+\left(1-\gamma_{2}\right) p_{1}-\gamma_{2} \tau_{1} \geq 0 \Rightarrow \\
& \tau_{2}-\left(1-\gamma_{2}\right) \tau_{1}-\gamma_{2} \tau_{1} \geq 0 \\
& \Rightarrow \tau_{2}-\tau_{1} \geq 0 \Rightarrow \#
\end{aligned}
$$

\section{Dann gilt}

$$
p_{1}<p_{2}
$$

und damit

$$
p_{2}-p_{1}>0
$$

Für den oben dargestellten Fall ergeben sich die folgenden Änderungsrichtungen ${ }^{16)}$ :

$$
\begin{aligned}
& \frac{d E_{1}}{d Y_{2}}=-\frac{u_{S S}^{1} E_{\tau}^{1}\left\{\left[\left(1-Y_{2}\right) u_{S S}^{2} E_{\tau}^{2}+a_{2}\left(\delta+a_{2}\right)\right] E_{2}+Y_{2} E_{\tau}^{2} a_{2}\left(\delta+a_{2}\right)\left(p_{2}-p_{1}\right)\right\}}{\Delta} \geq_{<} \quad(<0) \\
& \frac{d S_{1}}{d Y_{2}}=\frac{\left(\delta+a_{1}\right)\left\{\left[\left(1-Y_{2}\right) u_{S S}^{2} E_{\tau}^{2}+a_{2}\left(\delta+a_{2}\right)\right] E_{2}+Y_{2} E_{\tau}^{2} a_{2}\left(\delta+a_{2}\right)\left(p_{2}-p_{1}\right)\right\}}{\Delta} \geq_{0} \quad(>0) \\
& \frac{d p_{1}}{d Y_{2}}=\frac{u_{S S}^{1}\left\{\left[\left(1-Y_{2}\right) u_{S S}^{2} E_{\tau}^{2}+a_{2}\left(\delta+a_{2}\right)\right] E_{2}+Y_{2} E_{\tau}^{2} a_{2}\left(\delta+a_{2}\right)\left(p_{2}-p_{1}\right)\right\}}{\Delta} \geq_{0} \quad(<0) \\
& \frac{d \tau_{1}}{d Y_{2}}=-\frac{u_{S S}^{1}\left\{\left[\left(1-Y_{2}\right) u_{S S}^{2} E_{\tau}^{2}+a_{2}\left(\delta+a_{2}\right)\right] E_{2}+Y_{2} E_{\tau}^{2} a_{2}\left(\delta+a_{2}\right)\left(p_{2}-p_{1}\right)\right\}}{\Delta} \geq 0 \quad(>0)
\end{aligned}
$$$$
\frac{d E_{2}}{d Y_{2}}=\frac{\left.E_{\tau}^{2}\left\{\mid u_{S S}^{1} E_{\mathrm{\tau}}^{1}+a_{1}\left(\delta+a_{1}\right)\right]\left[\left(1-Y_{2}\right) u_{S S}^{2} E_{2}+a_{2}\left(\delta+a_{2}\right)\left(p_{2}-p_{1}\right)\right]-Y_{2} E_{2} a_{2}\left(\delta+a_{2}\right) u_{S S}^{1}\right\}}{\Delta} \geq_{<0} \quad\left(\geq_{<} 0\right)
$$

16) In Klammern sind die Änderungsrichtungen für den entgegengesetzten Fall $\left(p_{1}>p_{2}\right)$ angegeben. 


$$
\begin{aligned}
& \frac{d S_{2}}{d Y_{2}}=-\frac{\left(\delta+a_{2}\right)\left\{\left[u_{S S}^{1} E_{\mathrm{t}}^{1}+a_{1}\left(\delta+a_{1}\right)\right]\left[E_{2}-\left(1-Y_{2}\right) E_{\tau}^{2}\left(p_{2}-p_{1}\right)\right]+Y_{2} E_{\tau}^{2} E_{2} u_{S S}^{1}\right\}}{\Delta}<0 \quad\left(\geq_{<} 0\right) \\
& \frac{d p_{2}}{d Y_{2}}=-\frac{u_{S S}^{2}\left\{\left[u_{S S}^{1} E_{\tau}^{1}+a_{1}\left(\delta+a_{1}\right)\right]\left[E_{2}-\left(1-Y_{2}\right) E_{\tau}^{2}\left(p_{2}-p_{1}\right)\right]+Y_{2} E_{\tau}^{2} E_{2} u_{S S}^{1}\right\}}{\Delta}>0 \quad(\geq<0) \\
& \frac{d \tau_{2}}{d Y_{2}}=\frac{\left[u_{S S}^{1} E_{\tau}^{1}+a_{1}\left(\delta+a_{1}\right)\right]\left[\left(1-Y_{2}\right) u_{S S}^{2} E_{2}+a_{2}\left(\delta+a_{2}\right)\left(p_{2}-p_{1}\right)\right]-Y_{2} E_{2} a_{2}\left(\delta+a_{2}\right) u_{S S}^{1}}{\Delta} \geq_{0} \quad(\geq<0)
\end{aligned}
$$




\section{ANHANG 4.III: Standardsetzung}

1. Die Änderungen für Region 2

Für Region 2 muß folgendes Gleichungssystem betrachtet werden unter der Annahme, daß der Emissionsstandard bindend ist:

$$
\begin{aligned}
\left(1-\gamma_{2}\right) \mathbf{E}_{2}-\alpha_{2} S_{2} & =0 \\
\left(\delta+\alpha_{2}\right) p_{2}-u_{S}{ }_{S} & =0 \\
\tau_{2}+\left(1-\gamma_{2}\right) p_{2}-\lambda^{2}{ }_{2} & =0 \\
\mathbf{E}_{2}-E^{2}\left(\tau_{2}\right) & =0
\end{aligned}
$$

Man erhält

$$
\begin{aligned}
& {\left[\begin{array}{cccc}
-\alpha_{2} & 0 & 0 & 0 \\
-u_{S S}^{2} & \delta+\alpha_{2} & 0 & 0 \\
0 & 1-\gamma_{2} & 1 & -1 \\
0 & 0 & -E^{2}{ }_{\tau} & 0
\end{array}\right]\left[\begin{array}{l}
d_{2} \\
d p_{2} \\
d \tau_{2} \\
d \lambda_{2}{ }^{2}
\end{array}\right]=\left[\begin{array}{l}
\mathbf{E}_{2} \\
0 \\
p_{2} \\
0
\end{array}\right] d \gamma_{2}+\left[\begin{array}{l}
0 \\
-p_{2} \\
0 \\
0
\end{array}\right] d \delta+} \\
& +\left[\begin{array}{c}
S_{2} \\
-p_{2} \\
0 \\
0
\end{array}\right] d \alpha_{2}+\left[\begin{array}{c}
0 \\
0 \\
0 \\
E_{R}^{2}
\end{array}\right] d \mathbf{R}_{2}+\left[\begin{array}{c}
-\left(1-\gamma_{2}\right) \\
0 \\
0 \\
-1
\end{array}\right] d \mathbf{E}_{2}
\end{aligned}
$$


1. Die Änderungen für ein erhöhtes $\gamma_{2}$ :

$$
\begin{aligned}
& \frac{d S_{2}}{d Y_{2}}=\frac{-\mathbf{E}_{2}}{a_{2}}<0 \\
& \frac{d p_{2}}{d Y_{2}}=-\frac{u_{S S}^{2} E_{2}}{a_{2}\left(\delta+a_{2}\right)}>0 \\
& \frac{d \tau_{2}}{d Y_{2}}=0 \\
& \frac{d \lambda_{2}^{2}}{d Y_{2}}=-\frac{\left(1-Y_{2}\right) u_{S S}^{2} E_{2}}{a_{2}\left(\delta+a_{2}\right)}>0
\end{aligned}
$$

2. Die Änderungen für ein erhöhtes $\delta$ :

$$
\begin{aligned}
& \frac{d S_{2}}{d \delta}=0 \\
& \frac{d p_{2}}{d \delta}=\frac{-p_{2}}{\delta+a_{2}}>0 \\
& \frac{d \tau_{2}}{d \delta}=0 \\
& \frac{d \lambda_{2}^{2}}{d \delta}=-\frac{\left(1-\mathrm{Y}_{2}\right) p_{2}}{\delta+\mathrm{a}_{2}}>0
\end{aligned}
$$


3. Die Änderungen für ein erhöhtes $\alpha_{2}$ :

$$
\begin{aligned}
& \frac{d S_{2}}{d a_{2}}=\frac{-S_{2}}{a_{2}}<0 \\
& \frac{d p_{2}}{d a_{2}}=\frac{-\left|u_{S S}^{2} S_{2}+a_{2} p_{2}\right|}{a_{2}\left(\delta+a_{2}\right)}>0 \\
& \frac{d \tau_{2}}{d a_{2}}=0 \\
& \frac{d \lambda_{2}^{2}}{d a_{2}}=-\frac{\left(1-\gamma_{2}\right)\left|u_{S S}^{2} S_{2}+a_{2} p_{2}\right|}{a_{2}\left(\delta+a_{2}\right)}<0
\end{aligned}
$$

4. Die Änderungen für ein erhöhtes $\mathbf{R}_{2}$ :

$$
\begin{aligned}
& \frac{d S_{2}}{d \mathbf{R}_{2}}=0 \\
& \frac{d p_{2}}{d \mathbf{R}_{2}}=0 \\
& \frac{d \tau_{2}}{d \mathbf{R}_{2}}=\frac{-E_{\mathbf{R}}^{2}}{E_{\tau}^{2}}>0 \\
& \frac{d \Lambda_{2}^{2}}{d \mathbf{R}_{2}}=\frac{-E_{\mathbf{R}}^{2}}{E_{\tau}^{2}}>0
\end{aligned}
$$


5. Die Änderungen für ein erhöhtes $\mathbf{E}_{2}$ :

$$
\begin{aligned}
& \frac{d S_{2}}{d \mathbf{E}_{2}}=\frac{1-\mathrm{v}_{2}}{\mathrm{a}_{2}}>0 \\
& \frac{d p_{2}}{d \mathrm{E}_{2}}=\frac{\left(1-\mathrm{v}_{2}\right) u_{S S}^{2}}{\mathrm{a}_{2}\left(\delta+\mathrm{a}_{2}\right)}<0 \\
& \frac{d \tau_{2}}{d \mathrm{E}_{2}}=\frac{1}{E_{\tau}^{2}}<0 \\
& \frac{d \mathrm{\Lambda}_{2}^{2}}{d \mathrm{E}_{2}}=\frac{\mathrm{a}_{2}\left(\delta+\mathrm{a}_{2}\right)+\left(1-\mathrm{v}_{2}\right)^{2} E_{\mathrm{\tau}}^{2} u_{S S}^{2}}{\mathrm{a}_{2}\left(\delta+\mathrm{a}_{2}\right) E_{\tau}^{2}}<0
\end{aligned}
$$

\section{Die Änderungen für Region 1}

Für Region 1 erhalten wir ein analoges Gleichungssystem zu demjenigen autonomer Regionalbehörden [vgl. Anhang 4.I]:

$$
\begin{aligned}
E_{1}-\alpha_{1} S_{1} & =-\gamma_{2} E_{2} \\
\left(\delta+\alpha_{1}\right) p_{1}-u_{S}^{1} & =0 \\
\tau_{1}+p_{1} & =0 \\
E_{1}-E^{1}\left(\tau_{1}\right) & =0
\end{aligned}
$$

Totale Differentation ergibt 


$$
\begin{aligned}
& {\left[\begin{array}{cccc}
1 & -\alpha_{1} & 0 & 0 \\
0 & -u_{S S}^{1} & \delta+\alpha_{1} & 0 \\
0 & 0 & 1 & 1 \\
1 & 0 & 0 & -E_{\tau}^{1}
\end{array}\right]\left[\begin{array}{l}
d E_{1} \\
d S_{1} \\
d p_{1} \\
d \tau_{1}
\end{array}\right]=\left[\begin{array}{c}
-E_{2} \\
0 \\
0 \\
0
\end{array}\right] d \gamma_{2}+\left[\begin{array}{c}
0 \\
-p_{1} \\
0 \\
0
\end{array}\right] d \delta+} \\
& +\left[\begin{array}{c}
s_{1} \\
-p_{1} \\
0 \\
0
\end{array}\right] d \alpha_{1}+\left[\begin{array}{c}
0 \\
0 \\
0 \\
E_{R}^{1}
\end{array}\right] d \mathbf{R}_{1}+\left[\begin{array}{c}
-\gamma_{2} \\
0 \\
0 \\
0
\end{array}\right] d \mathrm{E}_{2}
\end{aligned}
$$

Die Systemdeterminante ist

$$
\Delta_{1}=u_{S S}^{1} E^{1} \tau+\alpha_{1}\left(\delta+\alpha_{1}\right)>0
$$

2.1 Die Änderungen für ein größeres $\gamma_{2}$ :

$$
\begin{aligned}
& \frac{d E_{1}}{d Y_{2}}=-\frac{E_{\tau}^{1} u_{S S}^{1} \mathbf{E}_{2}}{\Delta_{1}}<0 \\
& \frac{d S_{1}}{d Y_{2}}=\frac{\left(\delta+a_{1}\right) \mathbf{E}_{2}}{\Delta_{1}}>0 \\
& \frac{d p_{1}}{d Y_{2}}=\frac{u_{S S^{1}} \mathbf{E}_{2}}{\Delta_{1}}<0 \\
& \frac{d \tau_{1}}{d Y_{2}}=\frac{-u_{s S_{2}}^{1} \mathbf{E}_{2}}{\Delta_{1}}>0
\end{aligned}
$$


2.2 Die Änderungen für ein erhöhtes $\delta$ :

$$
\begin{aligned}
& \frac{d E_{1}}{d \delta}=\frac{E_{\mathrm{\tau}}^{1} a_{1} p_{1}}{\Delta_{1}}>0 \\
& \frac{d S_{1}}{d \delta}=\frac{E_{\tau}^{1} p_{1}}{\Delta_{1}}>0 \\
& \frac{d p_{1}}{d \delta}=\frac{-a_{1} p_{1}}{\Delta_{1}}>0 \\
& \frac{d \tau_{1}}{d \delta}=\frac{a_{1} p_{1}}{\Delta_{1}}<0
\end{aligned}
$$

2.3 Die Änderungen für ein erhöhtes $\alpha_{1}$ und $\mathbf{R}_{1}$ :

Hier ändert sich gegenüber dem autonomen Fall nichts, so da $ß$ auf eine Ableitung verzichtet wird [vgl. Anhang 4.I, 2.3 und 2.4].

2.4 Die Änderungen in Region 1 bei erhöhtem $\alpha_{2}$ und $\mathbf{R}_{2}$ :

Da der Emissionsstandard bindend ist, besteht keine Abhängigkeit von $\alpha_{2}$ und $\mathbf{R}_{2}$ für Region 1. Es gilt

$$
\frac{d E_{1}}{d \mathrm{a}_{2}}=\frac{d S_{1}}{d \mathrm{a}_{2}}=\frac{d p_{1}}{d \mathrm{a}_{2}}=\frac{d \tau_{1}}{d \mathrm{a}_{2}}=\frac{d E_{1}}{d \mathbf{R}_{2}}=\frac{d S_{1}}{d \mathbf{R}_{2}}=\frac{d p_{1}}{d \mathbf{R}_{2}}=\frac{d \tau_{1}}{d \mathbf{R}_{2}}=0
$$




\section{Kapitel 5: Interregionale Verflechtung durch Handel}

\section{PROBLEMSTELLUNG}

In diesem Teil der Arbeit wollen wir uns mit einem anderen Aspekt der regionalen Umweltpolitik beschäftigen; und zwar interessieren wir uns für die Frage, welche Auswirkungen Handelsbeziehungen zwischen Regionen auf die Umweltallokation haben. In diesem Zusammenhang können Regionen auch Staaten sein, die entweder autonom sind oder in einem Staatenverbund zusammengeschlossen sind. Die zwei Regionen sind Bestandteil eines "kleinen" Landes, das keinen Einfluß auf den Weltmarkt ausübt, für die Ökonomie sind die Weltmarktpreise vorgegeben und durch ihr Angebots- und Nachfrageverhalten ändert sich nichts für die anderen Beteiligten am Weltmarkt ${ }^{1}$. Die Struktur der Ökonomie ist in Schaubild 5.1 dargestellt. In diesem Kapitel soll von Diffusionsvorgängen abstrahiert werden, um die "reinen" Handelseffekte in dem intertemporalen Modellkontext isolieren zu können.

Da hier der Fall ohne Diffusion betrachtet wird, gilt

$$
\gamma_{1}=\gamma_{2}=0
$$

Aus den ökologischen Zustand-Funktionen wird-also

$$
\frac{d S_{i}}{d t}=E_{i}-a_{i} S_{i}^{2)}
$$

und die ökologischen Stationaritätsbedingungen sind

$$
S_{i}=\frac{1}{a_{i}} E_{i}
$$

Weiter gelten die in Kapitel 3, Abschnitt 1.3, beschriebenen Handelsbedingungen:

1) Für ein ähnliches Modell vgl. Asako (1979)

2) Vgl. Gleichung (3.4). 


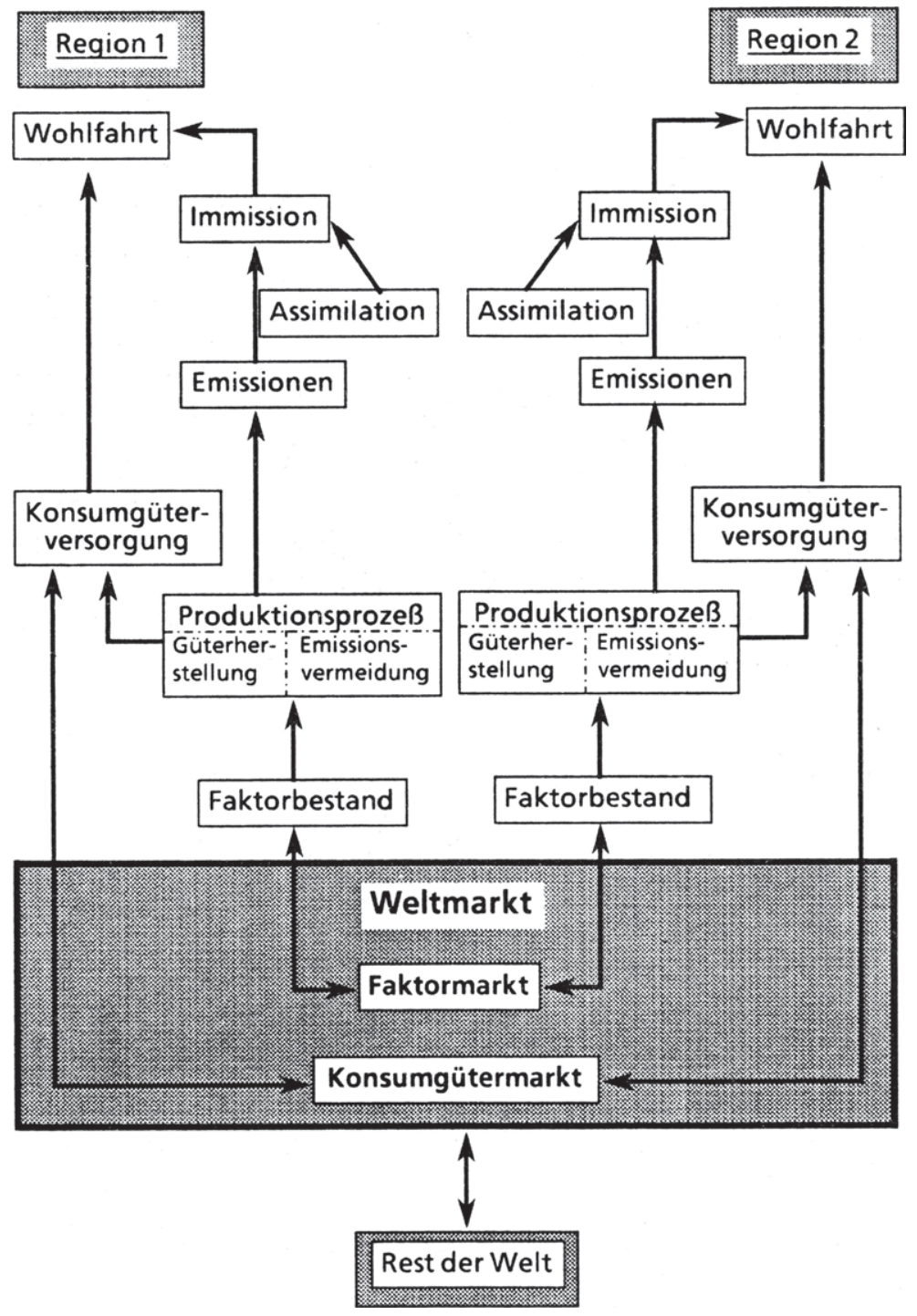

Schaubild 5.1: Die Struktur der Ökonomie 
Es gilt die Annahme, daß die Regionen Bestandteil eines "kleinen" Landes im Sinne der Außenhandelstheorie sind. Deshalb gilt

$$
q(t)=\mathbf{q} \quad \forall t \in[0, \infty),
$$

der Relativpreis ist konstant ${ }^{3)}$. Für die Zahlungsbilanzrestriktion gilt dann

$$
C_{i}-X_{i}+\mathbf{q}\left(R_{i}-\mathbf{R}_{i}\right)=0
$$

3) Liegt keine Diffusion zwischen den Regionen vor, und ist der Weltmarktpreis vorgegeben bzw. die Regionen können unbegrenzt Faktoren einkaufen, gibt es keinen Unterschied zwischen den autonomen Problemen und dem Problem der Zentralbehörde, wenn die Regionen gleich gewichtet werden, so daß hier nur das autonome Problem untersucht wird. 


\section{DIE OPTIMIERUNGSPROBLEME UND DIE OPTIMAL- BEDINGUNGEN}

Die Regionalbehörden der beiden Regionen sehen sich folgenden intertemporalen Optimierungsproblemen gegenüber $(i=1,2)$ :

$$
\begin{aligned}
& \operatorname{Max} W_{i}=\int_{0}^{\infty} u^{i}\left(C_{i}, S_{i}\right) e^{-\delta t} d t \\
& \text { u.d.B. (3.1) } f\left(R_{i}, E_{i}\right)-X_{i} \geq 0 \\
& \text { (3.2) } a_{i} R_{i}-E_{i} \geq 0 \\
& \text { (5.4) } C_{i}-X_{i}+q\left(R_{i}-R_{i}\right)=0 \\
& \text { (5.2) } \frac{d S_{i}}{d t}=E_{i}-a_{i} S_{i} \\
& X_{i}, C_{i}, R_{i}, E_{i} \geq 0 \forall t \in[0, \infty) \\
& S_{i}(0)=S_{i}{ }^{0} \text { gegeben } \\
& \delta \text { gegeben und positiv }
\end{aligned}
$$

Dies führt zu der Lagrange-Funktion in laufenden Werten

$$
\begin{aligned}
L^{i}=u^{i}\left(C_{i}, S_{i}\right)+ & p_{i}\left[E_{i}-\alpha_{i} S_{i}\right]+\lambda^{i}{ }_{1}\left[f^{i}\left(R_{i}, E_{i}\right)-X_{i}\right]+\lambda^{i}{ }_{2}\left[a_{i} R_{i}-E_{i}\right] \\
& +\lambda^{i}{ }_{3} C_{i}+\lambda^{i}{ }_{4} R_{i}+\lambda^{i}{ }_{5} E_{i}+\lambda^{i}{ }_{6} X_{i}+\mu^{i}\left[C_{i}-X_{i}+\mathbf{q}\left(R_{i}-\mathbf{R}_{i}\right)\right]
\end{aligned}
$$

Damit erhält man folgende Optimalbedingungen:

$$
L_{C}^{i}=u_{C}^{i}+\lambda^{i}{ }_{3}+\mu^{i}=0
$$$$
C_{i} \geq 0
$$

$$
L_{X}^{i}=-\lambda_{1}^{i}+\lambda_{6}^{i}-\mu^{i}=0
$$$$
x_{i} \geq 0
$$

$$
L_{R}^{i}=\lambda_{1}^{i} f_{R}{ }_{R}+\lambda^{i}{ }_{2} a_{i}+\lambda_{4}^{i}+\mathbf{q} \mu^{i}=0
$$$$
R_{i} \geq 0
$$

$$
L_{E}^{i}=p_{i}+\lambda_{1}^{i} f_{E}^{i}-\lambda_{2}^{i}+\lambda_{5}^{i}=0
$$$$
E_{i} \geq 0
$$

(v)

$$
L_{p}^{i}=\frac{d S_{i}}{d t}=E_{i}-a_{i} S_{i}
$$




$$
L_{S}^{i}=-\frac{d p_{i}}{d t}+\delta p_{i}=u_{S}^{i}-a_{i} p_{i}
$$

$$
\lambda_{1}^{i}\left[f^{i}\left(R_{i}, E_{i}\right)-X_{i}\right]=0
$$$$
\lambda_{1}^{i} \geq 0
$$

$$
\lambda_{2}^{i}\left[a_{i} R_{i}-E_{i}\right]=0
$$$$
\lambda_{2}^{i} \geq 0
$$

$$
\lambda_{3}^{i} C_{i}=0
$$$$
\lambda_{3}^{i} \geq 0
$$

$$
\lambda^{i}{ }_{4} R_{i}=0
$$$$
\lambda^{i}{ }_{4} \geq 0
$$

$$
\lambda_{5}^{i} E_{i}=0
$$$$
\lambda_{5}^{i} \geq 0
$$

$$
\lambda_{6}^{i} X_{i}=0
$$

$$
\mu^{i}\left[C_{i}-X_{i}+\mathbf{q}\left(R_{i}-\mathbf{R}_{i}\right)\right]=0
$$

Es gilt wieder, daß der Konsum in jedem Zeitpunkt positiv ist. Dann folgt

$$
\lambda_{3}^{i}=0 \quad \text { und } \quad u_{C}^{i}=-\mu^{i}>0
$$

Man kann eine Beziehung zwischen dem Konsum und dem Einsatz an Ressourcen und den Emissionen ableiten:

$$
C_{i}=f\left(R_{i}, E_{i}\right)-\mathbf{q}\left(R_{i}-\mathbf{R}_{i}\right)>0
$$

Es können zwei Fälle auftreten:

- Einmal verkauft die Regionalbehörde alle vorhandenen Ressourcen auf dem Weltmarkt, die im Produktionsprozeß eingesetzten Ressourcen sind Null:

$$
R_{i}=E_{i}=X_{i}=0 \text { und } C_{i}=\mathbf{q} \mathbf{R}_{i}
$$

der Gegenwert der verkauften Ressourcen wird konsumiert"). Gilt dies, dann muß folgende Bedingung erfüllt sein

$$
\left.f_{R}\right|_{R_{i}=0} \leq q^{5)}
$$

4) In Siebert (1983b) werden solche Fălle für erschöpfbare Ressourcen untersucht.

5) Dies folgt aus den Bedingungen (5.8.i), (5.8.ii) und (5.8.iii). 
Die Grenzproduktivität der ersten Einheit der Ressurce muß relativ niedrig sein und der Relativpreis $\mathbf{q}=\left(q_{R} / q_{X}\right)$ muß hoch sein. Es lohnt sich dann nicht, die Produktion aufzunehmen, da durch den Einsatz einer Ressourceneinheit im Produktionsprozeß der Konsum sinken würde ${ }^{6}$.

Dann gibt es in dieser Region kein Umweltproblem und damit muß keine Umweltpolitik betrieben werden.

Wir gehen davon aus, daß die Technologie in den Regionen so beschaffen ist, daß es sich nur dann nicht lohnt zumindest mit der Produktion zu beginnen, wenn der Relativpreis extrem hoch ist (im Grenzfall unendlich), d. h. man das Konsumgut $X$ praktisch umsonst erhält bzw. der Faktor extrem teuer ist. Dieser Fall wird im folgenden nicht weiter untersucht.

- Zum anderen kann gelten, daß der Ressourceneinsatz positiv ist und damit auch die Güterproduktion. Von dieser Konstellation wollen wir bei den folgenden Überlegungen ausgehen.

Da $R_{i}>0$ und $X_{i}>0$ gilt, folgt $\lambda_{4}^{i}=\lambda_{6}^{i}=0$. Deshalb gilt

$$
-\mu^{i}=u_{C}^{i}=\lambda_{1}^{i} \text {. }
$$

Aus Bedingung (5.8.iii) erhält man

$$
u_{C}^{i}\left(f_{R}^{i}-\mathbf{q}\right)+\lambda_{2}^{i} a_{i}=0
$$

und daraus folgt

$$
\lambda_{2}^{i}=-\frac{u_{C}^{i}}{a_{i}}\left(f_{R}-q\right) \quad \begin{cases}=0 & \text { für } E_{i}<a_{i} R_{i} \\ \geq 0 & \text { für } E_{i}=a_{i} R_{i}\end{cases}
$$

Durch Gleichung (5.9) ist eine Beziehung zwischen den Emissionen und dem Ressourceneinsatz definiert:

6) $\partial C_{i} / \partial R_{i}=f_{R}-\mathrm{q} \leq 0$ für $R_{i}=0$. 


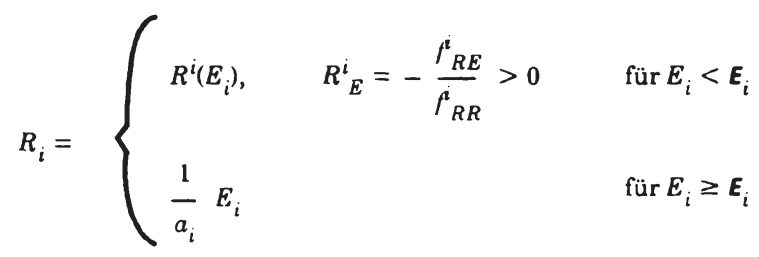

wobei $E_{i}$ diejenige Emissionsmenge ist, für die $f_{R}^{i}=q$ und $E_{i}=a_{i} R_{i}$ gilt.

Diese Funktion wird in Schaubild 5.2 graphisch abgeleitet. Im oberen Quadranten ist zum einen die Produktionsfunktion für jeweils konstante Emissionen $\mathrm{E}_{i}{ }^{\odot}<\mathrm{E}_{i}{ }^{\odot}<\mathrm{E}_{i}{ }^{\oplus}$ dargestellt. Zum anderen ist für konstanten Weltmarktpreis $\mathbf{q}$ die Budgetrestriktion für konstantes $\mathrm{C}_{i}{ }^{\odot}<\mathrm{C}_{i}{ }^{3}<\mathrm{C}_{i}{ }^{\odot}$ als Gerade eingezeichnet. Die Beziehung

$$
f_{R}=q \quad\left(\Leftrightarrow \lambda_{2}^{t}=0\right)
$$

ergibt sich aus der Gleichheit der Steigungen der Budgetrestriktion einerseits und der Produktionsfunktion andererseits. Durch die Kuppelproduktionsgrenze $E_{i}=a_{i} R_{i}$ können Emissionen, die oberhalb von $E_{i}$ liegen, nicht erreicht werden, da das Gesetz der Erhaltung der Masse hier berücksichtigt werden muß, so daß, wenn mehr als $\boldsymbol{R}_{i}$ Ressourcen eingesetzt werden, die Gleichheit der Grenzproduk tivität mit dem Relativpreis nicht mehr erreicht werden kann. Im Punkt $\left(\boldsymbol{E}_{i^{\prime}} \boldsymbol{R}_{i}\right)$ wird die Kuppelproduktionskapazität gerade ausgenutzt und es gilt die Gleichheit der Grenzproduktivität der Ressource mit dem konstanten Weltmarktpreis q.

Mit Hilfe der Beziehung (5.10) kann eine Funktion zwischen den Emissionen und dem Konsum abgeleitet werden, die im folgenden Konsum-Emissions-Funktion genannt wird: 


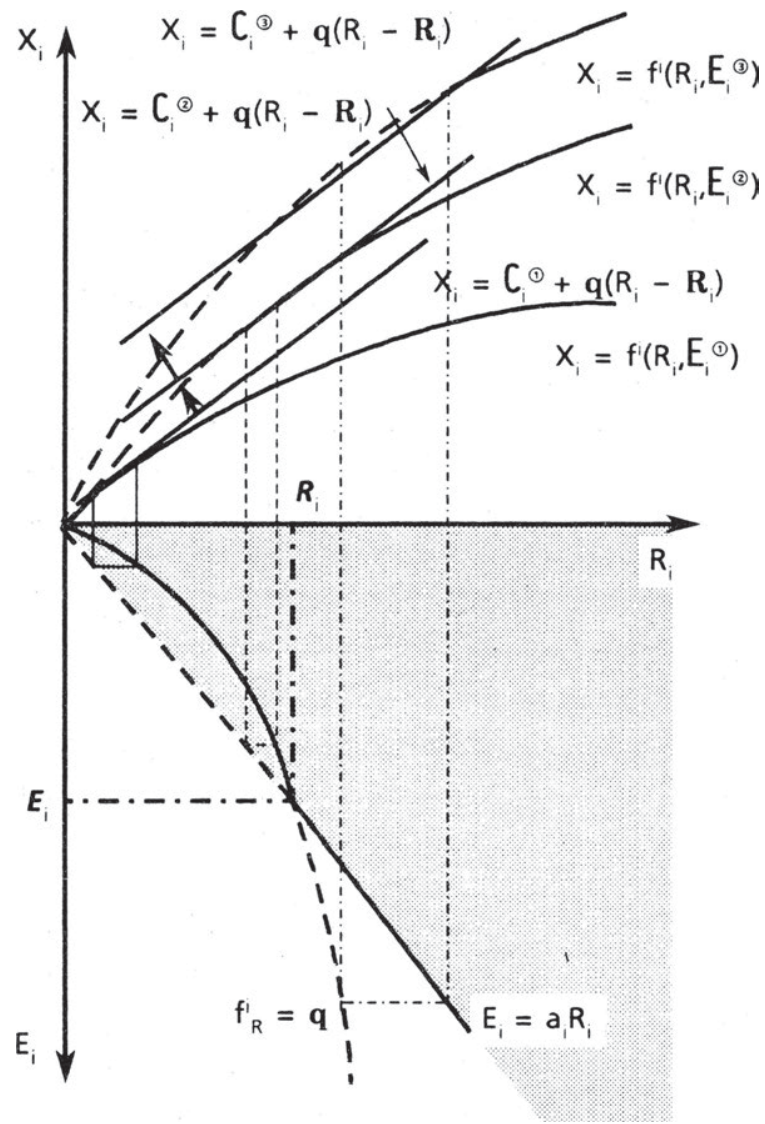

Schaubild 5.2: Die Beziehung zwischen $E_{i}$ und $R_{i}$ 


$$
\begin{aligned}
& C_{i}=C^{i}\left(E_{i}\right) \\
& \text { mit } C_{E}^{i}= \begin{cases}f_{E} & \text { für } E_{i}<E_{i} \\
\frac{1}{a_{i}}\left[f_{R}-\mathrm{q}\right]+f_{E} & \text { für } E_{i} \geq E_{i}\end{cases} \\
& C_{E E}^{\prime l}=\left\{\begin{array}{cc}
f_{E E}^{i}-\frac{\left(f_{R E}\right)^{2}}{f_{R R}}<0 & \text { für } E_{i}<E_{i} \\
\frac{1}{a_{i}^{2}} f_{R R}^{i}+\frac{2}{a_{i}} f_{E R}+f_{E E}<0 \text { f) für } E_{i} \geq E_{i}
\end{array}\right.
\end{aligned}
$$

Aus (5.12) kann ein Emissionsniveau $E_{i}{ }^{\max }$ abgeleitet werden, für das ein maximaler Konsum erreicht wird, danach wird bei weiterer Erhöhung der Emissionen (d. h. des Ressourceneinsatzes) der Konsum wieder geringer. Es gilt für $E_{i}>E_{i}^{\max }$

$$
\mathbf{q}>f_{R}+a_{i} f_{E}
$$

da die Ausgaben für eine weitere Ressourceneinheit größer sind als der zusätzliche Output dieser eingesetzten Ressourceneinheit. Daß die KonsumEmissions-Funktion in $E_{i}^{\max }$ ein Maximum hat, folgt aus $C_{E E}^{i}<0$ [vgl. Gleichung (5.12) und Fußnote 7].

Schaubild 5.3 zeigt die so gewonnene Konsum-Emissions-Funktion. Da ab $E_{i}=E_{i}$ die Beziehung $E_{i}=a_{i} R_{i}$ gilt, lohnt es sich für die Region $i$ nicht, mehr als $R_{i}^{\max }$ an Faktoren einzusetzen, da dann der Konsum fällt während die Emissionen steigen und dies gleichermaßen negative Wohlfahrtseffekte bewirkt. Damit ist ein maximaler Ressourceneinsatz definiert, der nicht überschritten wird und damit auch ein maximales Emissionsniveau $E_{i}^{\max }$.

7) Es gilt $f_{R R} f_{E E}^{i}-\left(f_{E R}^{i}\right)^{2}>0 \Leftrightarrow f_{R R}<\left[\left(f_{E R}^{i}\right)^{2} / f_{E E}^{i}\right] \Rightarrow$

$$
\begin{aligned}
& \left.\left[d^{2} C_{i}\right] d R_{i}^{2}\right]=f_{R R}+2 a_{i} f_{E R}+a_{i}^{2} f_{E E}^{i}<\left[\left(f_{E R}\right)^{2} / f_{E E}\right]+2 a_{i} f_{E R}+a_{i}^{2} f_{E E}= \\
& {\left[1 / f_{E E}^{i}\right]\left[\left(f_{E R}\right)^{2}+2 a_{i} f_{E R} f_{E E}+a_{i}^{2}\left(f_{E E}\right)^{2}\right]=\left[1 / f_{E E}^{i}\right]\left[f_{E R}^{i}+a_{i} f_{E E}^{i}\right]^{2} \leq 0 .}
\end{aligned}
$$




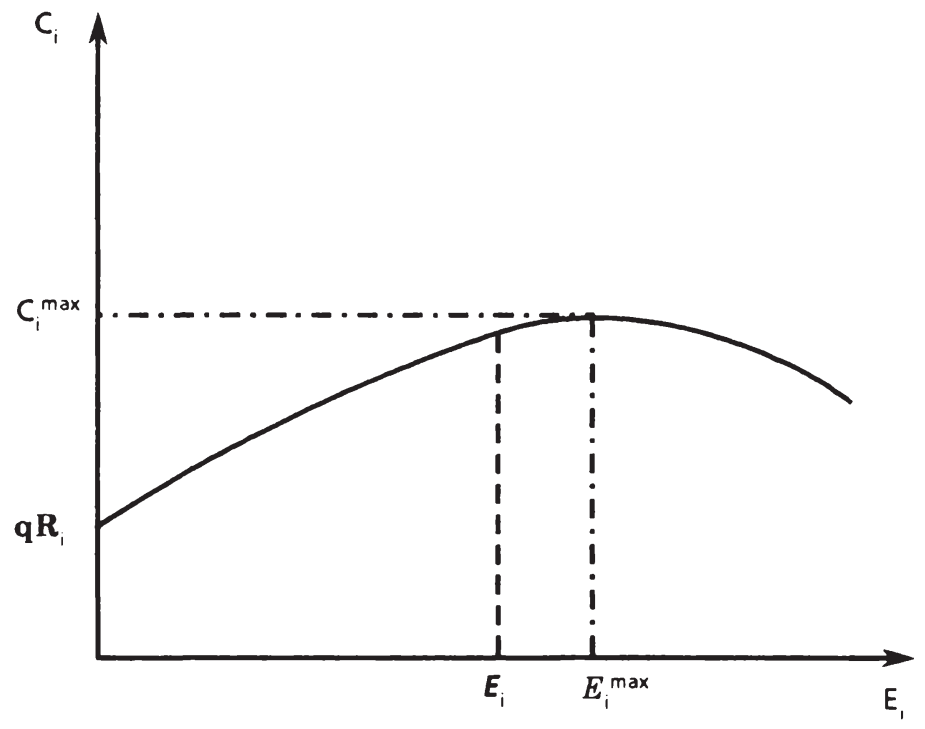

Schaubild 5.3: Die Beziehung zwischen $E_{i}$ und $C_{i}$

Die Steuerfunktionen können jetzt folgendermaßen abgeleitet werden ${ }^{8)}$

$$
\tau_{i}=u_{C}^{i} C_{E}^{i}
$$

Die optimale Steuer $\tau_{i}$ ist gerade der Betrag, der dem Beitrag der letzten Emissionseinheit zur Wohlfahrt im Zeitpunkt $t$ entspricht. Daraus kann folgende Funktion abgeleitet werden:

$$
\begin{aligned}
E_{i}=E^{i}\left(\tau_{i}\right) & \text { für } \tau_{i} \in\left[0, \tau_{i}{ }^{\prime}\right], \tau_{i}{ }^{\prime} \equiv u_{C}^{i} C^{i}{ }_{E} \mid E_{i}=0 \\
\text { mit } & E_{\mathrm{\tau}}^{i}=\frac{1}{u_{C C}^{i}\left(C_{E}^{i}\right)^{2}+u_{C}^{i} C_{E E}^{i}}<0 \quad\left(E_{i} \neq E_{i}\right)
\end{aligned}
$$

8) Vgl. die Gleichungen (3.25), (3.26) und (3.27). 
Die Beziehung zwischen der optimalen Steuer und dem Zukunftsschaden ist dann

$$
\tau_{i}= \begin{cases}\tau_{i}^{\prime} & \text { für } p_{i} \leq p_{i}^{\prime}, p_{i}^{\prime}=-\tau_{i}^{\prime} \\ -p_{i} & \text { für } p_{i} \in\left(p_{i}^{\prime}, 0\right) \\ 0 & \text { für } p_{i}=0\end{cases}
$$

In Schaubild 5.4 ist die Beziehung zwischen den Emissionen, der Steuer

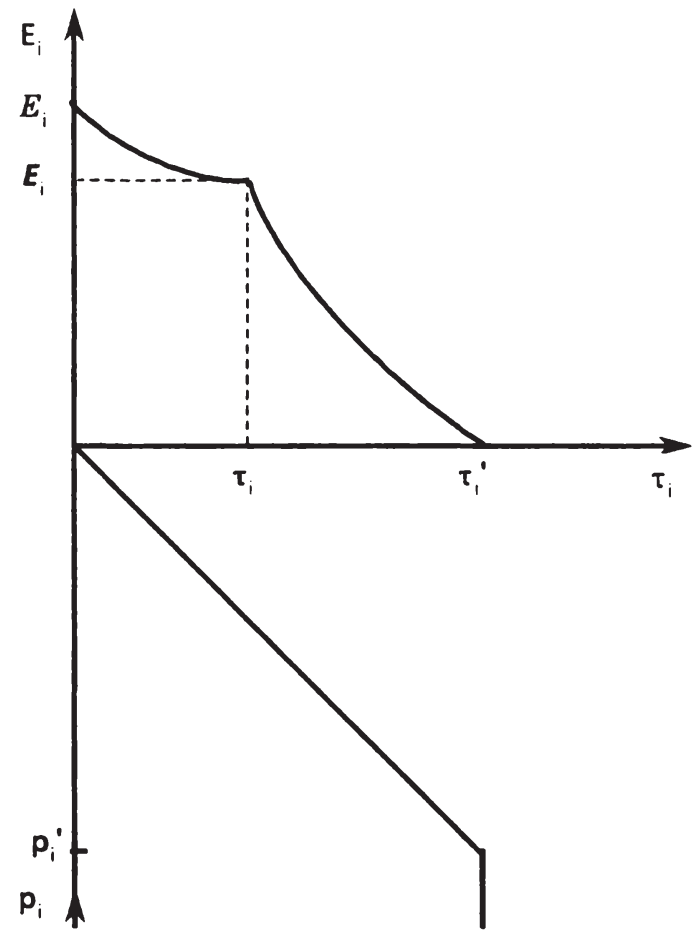

Schaubild 5.4: Die Beziehungen zwischen Emissionen, Steuern und Schattenpreis 
und dem Schattenpreis dargestellt. Im Punkt $\left(\tau_{i^{\prime}}, \boldsymbol{E}_{i}\right)$ hat die $E^{i}\left(\tau_{i}\right)$ Kurve einen Knick. Es kann gezeigt werden, daß bei der Annäherung von links an $\boldsymbol{\tau}_{i}$ die Kurve flacher verläuft als bei der Annäherung von rechts ${ }^{9}$.

\section{DIE ABLEITUNG DER OPTIMALPFADE UND DES GLEICHGEWICHTS}

Durch einsetzen von (5.14) und (5.15) in (5.8.v) und (5.8.vi) erhält man folgendes Differentialgleichungssystem ${ }^{10}\left(E_{i} \neq E_{l}\right)$ :

$$
\frac{d S_{i}}{d t}=E^{i}\left(-p_{i}\right)-a_{i} S_{i}
$$

$$
\frac{d p_{i}}{d t}=\left(\delta+a_{i}\right) p_{i}-u_{S}^{i}
$$

In Schaubild 5.5 ist der Ablauf der Annäherung an das Gleichgewicht für unterschiedliche Werte von $S_{i}{ }^{0}$ dargestellt. Im I. Quadranten ist die $E^{i}\left(\tau_{i}\right)$ Kurve eingezeichnet, im II. Quadranten die stationäre Ökozustand-Funktion, im III. Quadranten die Beziehung zwischen dem optimalen Ressourceneinsatz und dem optimalen Emissionsniveau. Im IV. Quadranten ist die Beziehung zwischen Steuer und Zukunftsschaden eingezeichnet. Im V.

9) Sei $E_{\mathrm{\tau}}^{i-}=\lim _{\mathrm{\tau}_{i} \rightarrow \tau_{i}, \tau_{i}<\tau_{i}} E_{\mathrm{\tau}}^{i}$ und $E_{\mathrm{\tau}}^{i+}=\lim _{\tau_{i} \rightarrow \tau_{i}, \tau_{i}>\tau_{i}} E_{\mathrm{r}}^{i}$ In $E_{i}=E_{i}$ gilt $E_{i}=a_{i} R_{i}$ und $f_{R}^{i}=\mathbf{q}$. Behauptung: $E_{\mathrm{\tau}}^{i-} \geq E_{\mathrm{\tau}}^{i+} \Leftrightarrow \frac{1}{E_{\tau}^{i+}} \geq \frac{1}{E_{\tau}^{i-}}$

$$
\begin{aligned}
& \left.\Leftrightarrow u_{C C}^{i}\left(f_{E}^{i}\right)^{2}+u_{C}^{i} f_{E E}^{i}-u_{C}^{i} \frac{\left(f_{E R}^{i}\right)^{2}}{f_{R R}^{i}} \geq u_{C C}^{i}\left[f_{E}^{i}+\frac{1}{a_{i}}\left(f_{R}^{i}-q\right)\right]^{2}+u_{C}^{i} \mid \frac{1}{a_{i}^{2}} f_{R R}^{i}+\frac{2}{a_{i}} f_{R E}^{i}+f_{E E}^{i}\right] \\
& \Leftrightarrow 0 \geq u_{C}^{i}\left[\frac{1}{a_{i}^{2}} f_{R R}^{i}+\frac{2}{a_{i}} f_{R E}^{i}+\frac{\left(f_{E R}^{i}\right)^{2}}{f_{R R}^{i}}\left|\Leftrightarrow 0 \geq \frac{1}{f_{R R}^{i}}\right| \frac{1}{a_{i}} f_{R R}^{i}+\left.f_{R E}^{i}\right|^{2}\right. \text { q.e.d. }
\end{aligned}
$$

10) Unter der Annahme, da $\beta E_{i}>0$ gilt $\forall t \in[0, \infty)$. Zur Lösbarkeit dieses Differentialgleichungssystems vgl. die Ausführungen zum Grundmodell in Kapitel 3. 
Quadranten wird das Steady-State für unterschiedliche Bewertungen des Immissionsbestandes, d. h. unterschiedlichen $d p_{i} / d t=0$ Funktionen, abgeleitet. Ein Steady-State ist im Schnittpunkt der $d p_{i} / d t=0$ Kurve mit der $d S_{i} / d t=0$ Kurve erreicht. Wird der Zukunftsschaden durch eine Immissionseinheit hoch bewertet, dann verläuft die $d p_{i} / d t=0$ Kurve steil, so da $B$ das Steady-State bei niedrigem $p_{i}$ und niedrigem $S_{i}$ liegt. Ist der Zukunfts-

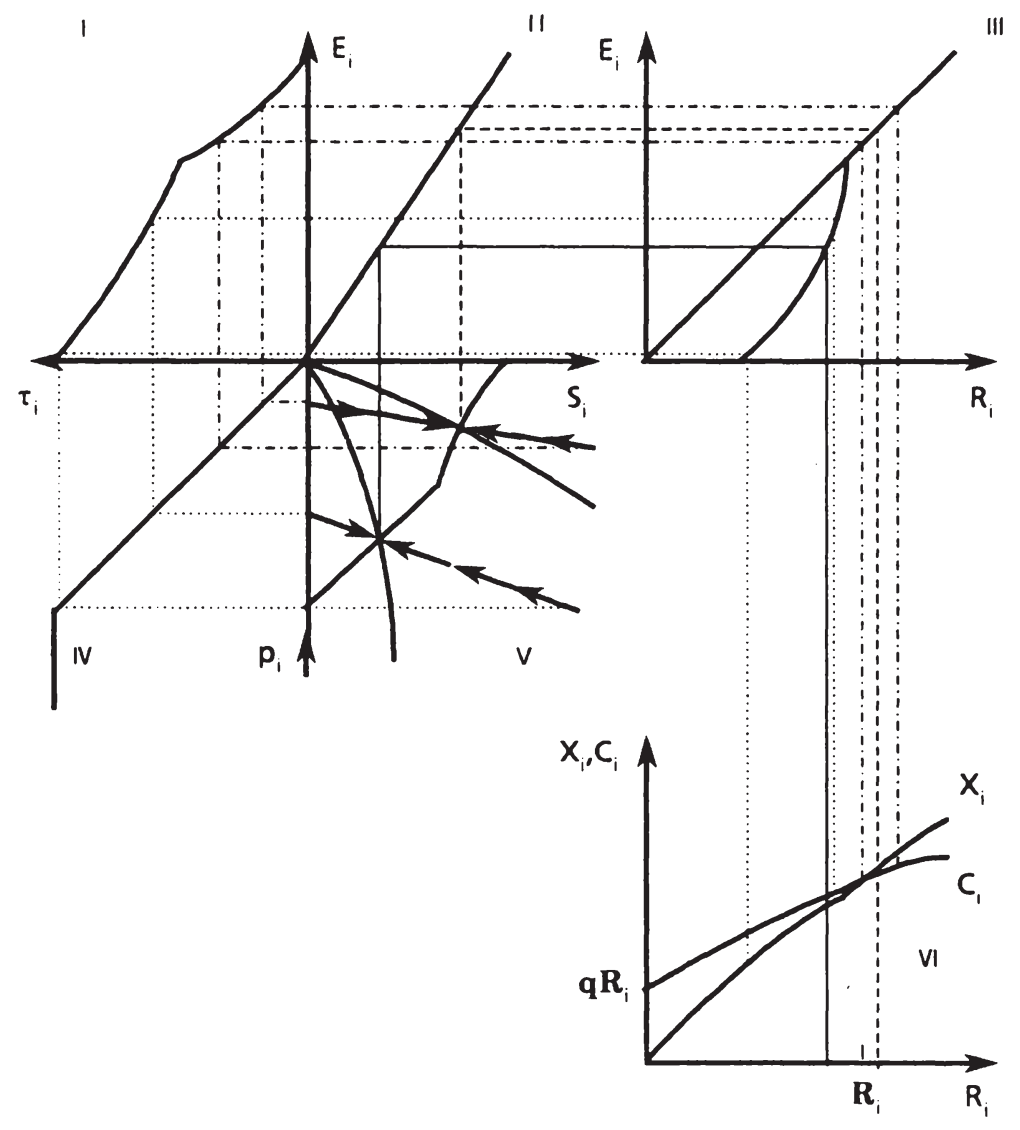

Schaubild 5.5: Die Optimaltrajektorien mit Handel 
schaden jedoch niedrig bewertet, dann verläuft die $d p_{i} / d t=0$ Kurve flacher und das Gleichgewicht $\left(p_{i}^{\infty}, S_{i}^{\infty}\right)$ ist bei hohem Immissionsbestand und niedriger Bewertung desselben $\left(p_{i}^{\infty}\right.$ ist groß). Im VI. Quadranten schließlich sind die optimalen Konsum- und Produktionsfunktionen eingezeichnet.

Ist $S_{i}{ }^{0}>S_{i}{ }^{\infty}$, dann wird am Anfang wenig emittiert. Es kann vorkommen, daß ein Teil des Ressourcenbestandes exportiert wird und ein Teil der Konsumgüter importiert wird. Die Emissionen und der Ressourceneinsatz steigen im Zeitablauf und es kann sein, daß die Region vom Ressourcenanbieter zum Ressourcennachfrager wird.

Ist $S_{i}{ }^{0}<S_{i}^{\infty}$, es wurde bisher noch wenig produziert bzw. wenig verschmutzt, kann zu Beginn des Planungszeitraumes viel emittiert werden, da die Immissionen sehr niedrig sind und die Aufnahmekapazität des Ökosystems groß ist. Im Zeitablauf steigen die Immissionen solange, bis das Steady-State erreicht ist. Deshalb müssen die Emissionen von ihrem hohen Niveau herabgesetzt werden, die Emissionen sinken also im Zeitablauf und deshalb sinkt auch die Güterproduktion, so daß die Region von einem Ressourcenimporteur zu einem Ressourcenexporteur werden kann. Die Umweltpolitik hat demnach auf das Marktverhalten der Regionen starken Einfluß.

Im Steady-State gilt

$$
\frac{u_{S}^{i}}{\delta+a_{i}}=-u_{C}^{i} C_{E}^{i}
$$

d.h. die Regionalregierung ist indifferent durch eine zusätzliche Emissionseinheit die Immissionen zu erhöhen und damit den Nutzen zu mindern und den Nutzen durch eine zusätzliche Emissionseinheit zu mehren, indem mehr Konsumgut produziert wird. 


\section{DIE AUSWIRKUNGEN VON PARAMETERÄNDERUNGEN AUF DAS STEADY-STATE}

Betrachten wir auch hier die Auswirkungen von Parameteränderungen auf das Steady-State. Die Änderungsrichtungen sind in folgender Tabelle zusammengefaßt. Die Ableitung der Resultate erfolgt im Anhang zu diesem Kapitel.

\begin{tabular}{|c|ccccc|}
\hline & $d E_{i}$ & $d S_{i}$ & $d p_{i}$ & $d \tau_{i}$ & $d C_{i}$ \\
\hline$d \delta$ & $\oplus$ & $\oplus$ & $\oplus$ & $\Theta$ & $\oplus$ \\
$d \boldsymbol{\alpha}_{i}$ & $\oplus$ & $?$ & $\oplus$ & $\Theta$ & $\oplus$ \\
$d \mathbf{R}_{i}$ & $\Theta$ & $\Theta$ & $\oplus$ & $\Theta$ & $\oplus$ \\
$d \mathbf{q}$ & $\Theta$ & $\Theta$ & $\oplus$ & $\Theta$ & $?$ \\
\hline
\end{tabular}

Tabelle 5.1: Die Effekte von Parameteränderungen

\subsection{DIE ABHÄNGIGKEIT DES STEADY-STATES VON DER SOZIALEN DISKONTRATE UND VON DEN ASSIMILATIONSKOEFFIZIENTEN}

Die Änderungen des Steady-States bei einer Änderung der sozialen Diskontrate sind eindeutig: Je höher die Diskontrate ist, desto mehr Faktoren werden auf dem Weltmarkt gekauft und eingesetzt und desto mehr wird deshalb emittiert, damit steigen auch die Immissionen, und die optimale Steuer sinkt, während der Konsum steigt. Wird also die Wohlfahrt der Zukunft niedrig bewertet, wird weniger Gewicht auf die Umweltqualität gelegt, die den zukünftigen Nutzen beeinflußt, sondern es wird in der Gegenwart mehr konsumiert, dies erhöht den gegenwärtigen Nutzen.

Je höher der Assimilationskoeffizient in einer Region ist, desto mehr wird emittiert und damit produziert und desto mehr wird konsumiert. Die optimale Steuer ist umso kleiner und der bewertete Schaden aus der Emissionstätigkeit ist umso niedriger je größer die Assimilationsfähigkeit ist. Über die Änderung des optimalen Immissionsbestandes kann wiederum keine eindeutige Aussage gemacht werden. 
Es gilt

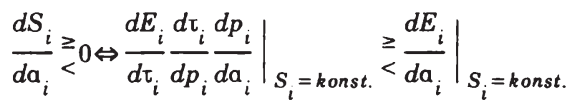

Diese Bedingung ist äquivalent zu der in Kapitel 3 abgeleiteten Bedingung (3.36), so daß hier auf eine Diskussion verzichtet werden kann. Die Abhängigkeit der Immissionen vom Assimilationskoeffizienten ist damit in der Richtung unabhängig von der Mobilität der Güter und Faktoren. [Vgl. hierzu auch Schaubild 3.6].

Wie ein Vergleich der Gleichungssysteme zeigt, sind die Ergebnisse, die wir für den autarken Fall in Kapitel 3 erhalten haben, ohne Einschränkung auf die Analyse mit Güter- und Faktormobilität übertragbar. Da keine direkten Rückwirkungen zwischen den Regionen bestehen, sondern diese hier nur über Handelsbeziehungen indirekt verbunden sind (vernachlässigt man die identische Zeitpräferenzrate, die ebenfalls eine schwache Verbundenheit ausdrückt), ist es nicht verwunderlich, daß die Ergebnisse hier so vollkommen äquivalent sind.

\subsection{UNTERSCHIEDLICHE FAKTORAUSSTATTUNGEN}

Untersuchen wir jetzt die Frage, ob es in der Ökonomie zu einer Angleichung der Emissionsteuern in den Regionen kommt, wenn die Faktorausstattungen in den Regionen unterschiedlich sind bei sonst identischen Regionen.

Sei

$$
\mathbf{R}_{1}>\mathbf{R}_{2}
$$

Die Konsum-Emissions-Funktion der Region 1 verläuft jeweils oberhalb der Konsum-Emissions-Funktion der Region 2, während die Begrenzung der Emissionen in beiden Regionen identisch ist, so daß für beide Regionen $\boldsymbol{R}_{i}$ und $R_{i}^{\text {max }}$ gleich ist und damit auch $E_{i}$ und $E_{i}^{\text {max }}$. Für die $E^{i}\left(\tau_{i}\right)$-Funktionen ergibt sich für größeres $\mathbf{R}_{i}$ eine Verschiebung nach innen, wobei für $\tau_{i}$ $=0$ die beiden Funktionen einen gemeinsamen Punkt haben. Im folgenden Schaubild ist diese Situation dargestellt. Der Ressourceneinsatz, und 


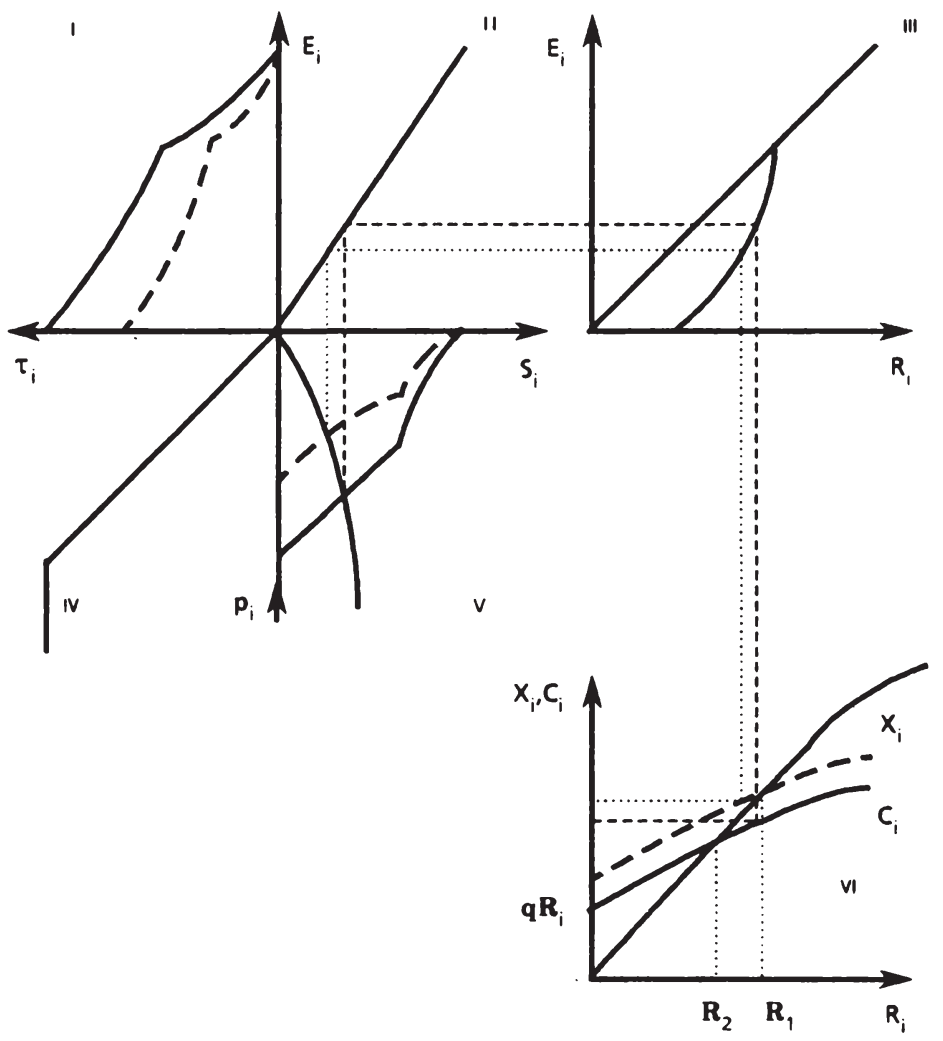

Schaubild 5.6: Unterschiedliche Ressourcenausstattung und Handel

damit auch die Emissionen, ist in der Region niedriger, in der mehr Faktoren vorhanden sind, d. h. Region 1, als in Region 2. Auch die Immissionen sind im Steady-State niedriger, da weniger emittiert wird. Dann gilt aber, da für beide Regionen die gleiche $d p_{i} d t=0$ Kurve gilt, daß $p_{1}^{\infty}$ größer ist als $p_{2}{ }^{\infty}$, wegen der negativen Steigung der $d p_{i} / d t=0$ Kurve und deshalb auch - abgesehen von Randlösungen ${ }^{11)}$ - daß die optimale Steuer $\tau_{1}{ }^{\infty}$ der

11) Es wird hier von der Annahme ausgegangen, $\operatorname{da} B E_{i}>0$ ist $(i=1,2) \forall t \in[0, \infty)$. 
Region 1 kleiner ist als die optimale Steuer $\tau_{2}{ }^{\infty}$ in Region 2. Der Konsum in Region 1 ist im Steady-State größer als in Region 2, jedoch wird die Konsumgüterversorgung tendenzmäßig immer mehr durch Importe vorgenommen.

Zusammenfassend läßt sich sagen, daß je größer die Ausstattung der Region mit Ressourcen ist, desto weniger wird in der Region produziert. Durch Berücksichtigung der Umweltqualität in den Wohlfahrtsfunktionen der Regionen sinkt die Bereitschaft, das eigene Land zu verschmutzen, wenn man relativ günstig auf dem Weltmarkt einkaufen kann. Eine Angleichung der Emissionssteuer ist im Steady-State jedoch nicht gegeben, da für identische optimale Steuersetzung der Zukunftsschaden unabhängig von der Höhe des Immissionsbestandes immer gleich bewertet werden müßte. Dadurch, daß ein höherer Immissionsbestand die Wohlfahrt überproportional senkt ${ }^{12}$, sind identische Steuern ausgeschlossen.

Im Vergleich zu dem in Kapitel 3 untersuchten Fall autarker Regionen, gibt es hier einen interessanten Unterschied. In Kapitel 3 wurde gezeigt, daß die Emissionen bei höherem Ressourcenbestand steigen [vgl. Anhang Kapitel 3]. Hier jedoch sinken die Emissionen für höheren Faktorbestand [vgl. die Ableitung im Anhang].

Dieser Unterschied kann folgendermaßen erklärt werden:

Bei autarken Regionen können höchstens $\mathbf{R}_{i}$ Faktoren eingesetzt werden und es ist optimal, dies auch zu tun. Wird jetzt der Faktorbestand größer, ist es weiterhin optimal, alle Faktoren im Produktionsprozeß einzusetzen. Dadurch wird die Grenzproduktivität der Emissionen größer $\left(f_{E R}>0\right)^{13}$. Durch die höhere Grenzproduktivität lohnt es sich für die Region, mehr zu emittieren und damit überproportional mehr zu produzieren.

Sind die Regionen jedoch an einen Weltmarkt angebunden, "verbessert" sich durch den höheren Faktorbestand die Handelsposition der Region. Bei einem konstanten Weltmarktpreis kann die Region aufgrund der Zah-

12) d. h. $u_{S S}^{i}<0$. Für $u_{S S}^{i}=0$ ist $p_{1}=p_{2}=\left[u_{S}^{i} d\left(\delta+\alpha_{i}\right)\right]=$ konst., unabhängig davon, wie groß $S_{i}$ ist.

13) Falls dies nicht der Fall ist $\left(\dot{f}_{E \mathbf{R}}=0\right)$, dann sinken auch im autarken Fall die Emissionen mit steigendem Faktorbestand. Vgl. Fußnote 46, Kapitel 3. 
lungsbilanzrestriktion bei höherem Faktorbestand und unveränderter Produktion mehr konsumieren. Diese Aussage entspricht dem in der Aussenhandelstheorie wohlbekannten Rybczinski-Theorem, wonach "bei konstanten Güterpreisen und bei Zunahme eines Faktors (.) der Output desjenigen Gutes zu(nimmt), das den zunehmenden Faktor intensiv nutzt. Der Output des anderen Gutes nimmt ab" [Siebert $(1977,59)]^{14}$.

Der entscheidende Unterschied liegt in der Art der Faktorbegrenzung. Im autarken Fall erfolgt sie direkt über eine Mengenbegrenzung, während mit Handelsbeziehungen die Restriktion über den Zahlungsbilanzausgleich erfolgt und damit eine Wertbegrenzung gegeben ist; dann hängt der Faktoreinsatz entscheidend von der Höhe des Relativpreises ab und die Mengenbegrenzung ist von zweitrangiger Bedeutung.

\subsection{Die AusWiRKungen DER HÖHE DES RElativPREiSes AUF DAS STEADY-STATE}

Der Relativpreis q ist ein Parameter, auf den die betrachtete Ökonomie keinen Einfluß hat, da ihre Angebots- und Nachfragemengen klein sind im Vergleich zu den anderen Marktteilnehmern, dem "Rest der Welt". Eine Variation des Relativpreises zeigt dann die Abhängigkeit der Ökonomie von Gegebenheiten, die außerhalb ihres Gebietes liegen.

Es gilt, daß die Emissionen mit steigendem Relativpreis fallen, die Immissionen sinken, die Steuer sinkt und der Schattenpreis steigt, während über die Konsumänderungen keine eindeutige Aussage abgeleitet werden kann.

Ist Region $i$ in der Ausgangslage Ressourcenimporteur, d. h. $C^{i}{ }_{\mathbf{q}}=$ $-\left(R_{i}-R_{i}\right)<0$, dann ist die Änderung des Konsums bei steigendem Relativpreis eindeutig: der Konsum sinkt. Durch die Verteuerung des Faktors muß bei konstanter Produktion mehr Konsumgut exportiert werden, damit die Faktormenge konstant bleibt (dies folgt aus der Zahlungsbilanzrestriktion (5.5)), bzw. bei konstantem Importwert $\mathrm{q} R_{i}$ sinkt die Produktion, da weniger Faktoren zur Verfügung stehen.

14) Vgl. hierzu auch Asako (1979), der in einem anderen Modellzusammenhang zu einer ãquivalenten Aussage kommt. 
Ist Region $i$ in der Ausgangslage Ressourcenexporteur, ist die Auswirkung des steigenden Relativpreises nicht mehr eindeutig; dann gilt

$$
\frac{d C_{i}}{d \mathbf{q}} \geq 0 \Leftrightarrow \frac{u_{S S}^{i}}{a_{i}\left(\delta+a_{i}\right)} \frac{C_{\mathbf{q}}^{i}}{C_{E}^{i}}>-u_{C}^{i}\left(\frac{C_{E E}^{i} C_{\mathbf{q}}^{i}}{C_{E}^{i}}-C_{E \mathbf{q}}^{i}\right)
$$

Dies ist äquivalent zu

$$
\left|\frac{d \tau_{i}}{d p_{i}} \frac{d p_{i}}{d S_{i}} \frac{d S_{i}}{d E_{i}} \frac{d E_{i}}{d \mathbf{q}}\right|_{C_{i}=\text { konst. }}\left|\underset{<\mid}{\geq \mid \frac{d \tau_{i}}{d \mathbf{q}}}\right|_{C_{i}=\text { konst. }} \mid
$$

Es wird die Größe zweier Effekte betrachtet, die für konstantes $C_{i}$ auf die Steuer $\tau_{i}$ wirken durch ein erhöhtes q. Effekt (1) gibt die Änderung der Steuer für konstanten Konsum an, die sich über die Änderung der Immissionen ergibt, während Effekt (2) die Änderung der Steuer über die Änderung der Emissionen für konstantes $C_{i}$ angibt. Ist der Faktorbestand der Region $i$ genügend groß bzw. die Umweltpolitik sehr streng, dann steigt durch den steigenden Relativpreis auf dem Weltmarkt der Gleichgewichtskonsum dieser Region. Dieser Fall ist in Schaubild 5.7 dargestellt.

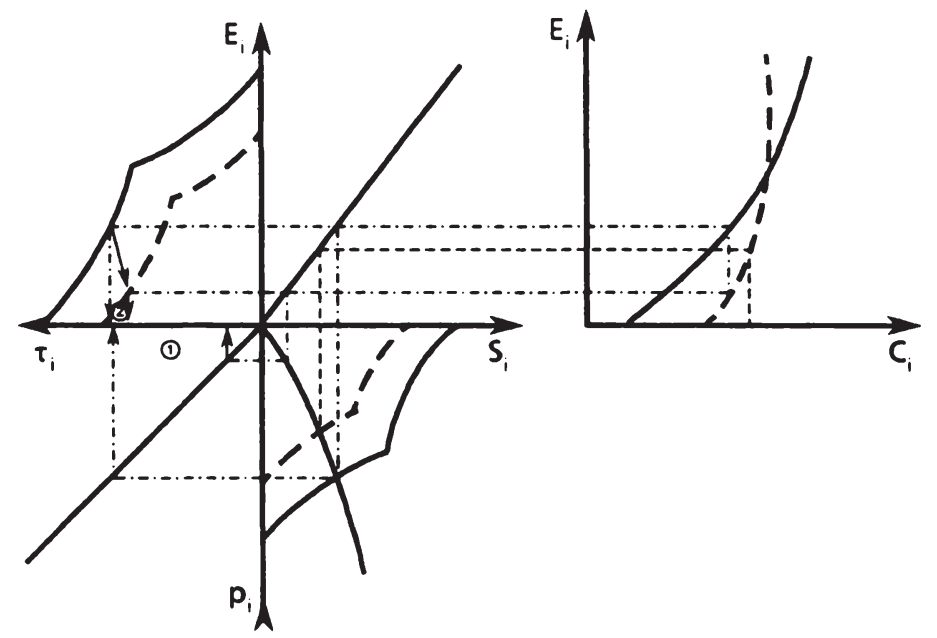

Schaubild 5.7: Die Änderung des Konsums für eine Relativpreiserhöhung 
Daß Region $i$ für steigenden Konsum Ressourcenexporteur ist, ist eine notwendige aber nicht hinreichende Bedingung. Ohne Umweltpolitik wäre die Bedingung hinreichend, jedoch kann bei Umweltpolitik der Wohlfahrtseffekt durch die Preissteigerung der Ressourcen überkompensiert werden durch den Wohlfahrtseffekt durch die Verminderung der Immissionen, so daß es sich für Region $i$ lohnen kann, weniger zu konsumieren, weniger zu emittieren und damit die Immissionen stärker zu senken.

\section{ZUSAMMENFASSUNG}

In diesem Kapitel wird das Grundmodell des Kapitels 3 dahingehend modifiziert, da $\beta$ Güter- und Faktorströme mit in die Analyse mit einbezogen werden können. Es wird gezeigt, daß die Umweltpolitik auf das Marktverhalten der Regionen starken Einfluß hat und umgekehrt.

Die Werte der Steady-State-Variablen werden entscheidend von den Handelsbeziehungen beeinflußt. Die in Kapitel 3 abgeleiteten Änderungsrichtungen bei autarken Regionen können hier als Referenzpunkt der Auswirkungen der Parameter auf das Steady-State genommen werden. Im einzelnen ergibt sich:

(i) Die Eigenschaften der Ökosysteme $\left(\alpha_{i}\right)$ und die Höhe der sozialen Diskontrate $\delta$ sind sowohl ohne als auch mit Güter- und Faktormobilität in ihren Auswirkungen auf die Steady-State-Variablen äquivalent.

(ii) Unterschiede ergeben sich jedoch bei den Auswirkungen der Höhe des Faktorbestandes auf das Steady-State. Sind die Regionen durch Handelsbeziehungen an den Weltmarkt angebunden, existiert neben der Mengenbegrenzung für den Einsatz des Faktors, die nur von zweitrangiger Bedeutung ist, eine wertmäßige Begrenzung des Faktoreinsatzes, die von entscheidender Bedeutung für das Steady-State ist. Die Rolle, die der Faktorbestand im autarken Fall spielt, wird bei den Handelsbeziehungen teilweise von dem konstanten Relativpreis übernommen. Es wird ein analoges Resultat zum Rybczinski Theorem der Außenhandelstheorie erzielt. 


\section{Anhang 5}

Totale Differentiation des Gleichungssystems

$$
\begin{aligned}
E_{i}-\alpha_{i} S_{i} & =0 \\
\left(\delta+\alpha_{i}\right) p_{i}-u_{S}^{i} & =0 \\
\tau_{1}+p_{i} & =0 \\
\tau_{i}-E^{i}\left(\tau_{i}\right) & =0
\end{aligned}
$$

führt zu der Matrixgleichung

$$
\left[\begin{array}{cccc}
1 & -\alpha_{i} & 0 & 0 \\
0 & -u_{S S}^{i} & \delta+\alpha_{i} & 0 \\
0 & 0 & 1 & 1 \\
1 & 0 & 0 & -E_{\tau}^{i}
\end{array}\right]\left[\begin{array}{c}
d E_{i} \\
d S_{i} \\
d p_{i} \\
d \tau_{i}
\end{array}\right]=\left[\begin{array}{c}
S_{i} \\
-p_{i} \\
0 \\
0
\end{array}\right] d \alpha_{i}+\left[\begin{array}{c}
0 \\
p_{i} \\
0 \\
0
\end{array}\right] d \delta+\left[\begin{array}{c}
0 \\
0 \\
0 \\
E^{i}
\end{array}\right] d \mathbf{R}_{i}+\left[\begin{array}{c}
0 \\
0 \\
0 \\
E^{i} \mathbf{q}
\end{array}\right] d \mathbf{q}
$$

wobei

$$
E_{\mathbf{q}}^{i}=-\frac{u_{C C}^{i} C_{E}^{i} C_{\mathbf{q}}^{i}+u_{C}^{i} C_{E \mathbf{q}}^{i}}{u_{C C}^{i}\left(C_{E}^{i}\right)^{2}+u_{C}^{i} C_{E E}^{i}}
$$

und

$$
E_{\mathbf{R}}^{i}=-\frac{u_{C C}^{i} C_{E}^{i} C_{\mathbf{R}}^{i}}{u_{C C}^{i}\left(C_{E}^{i}\right)^{2}+u_{C}^{i} C_{E E}^{i}}<0
$$

Weiter gilt

$$
\begin{aligned}
& C_{E \mathbf{q}}^{i}= \begin{cases}\frac{f_{R E}}{f_{R R}}<0 & \text { für } E_{i}<E_{i} \\
-\frac{1}{a_{i}} & \text { für } E_{i} \geq E_{i}\end{cases} \\
& C_{\mathbf{q}}^{i}=-M_{i}=-\left(R_{i}-\mathbf{R}_{i}\right)\left\{{ }_{=}\right\}_{0}
\end{aligned}
$$




$$
C_{\mathbf{R}}^{i}=\mathbf{q}
$$

Die Systemdeterminante ist

$$
\Delta_{i}=\alpha_{i}\left(\delta+\alpha_{i}\right)+u_{S S}^{i} E_{\tau}^{i}>0
$$

1. Die Abhängigkeit der Steady-State-Variablen von $\alpha_{i}$ und $\delta$

Ein Vergleich des Gleichungssystems dieses Anhangs mit demjenigen in Kapitel 3 zeigt, daß sich die Gleichungssysteme nur in der betragsmäßigen Höhe von $E^{i}{ }_{\tau}$ unterscheiden. Da jedoch auch das Vorzeichen der Ableitung der $E^{i}\left(\tau_{i}\right)$-Funktionen identisch negativ ist, kann hier auf eine Auflistung der Ableitungen verzichtet werden [vgl. Anhang zu Kapitel 3, 1. und 2.].

Die Änderung des Konsums ergibt sich für $\alpha_{i}$ folgendermaßen:

$$
\frac{d C_{i}}{d a_{i}}=C_{E}^{i} \frac{d E_{i}}{d a_{i}}=\frac{C_{E}^{i} E_{\tau}^{i}\left[u_{S S}^{i} S_{i}+a_{i} p_{i}\right]}{\Delta_{i}}>0
$$

und bei $\delta$

$$
\frac{d C_{i}}{d \delta}=C_{E}^{i} \frac{d E_{i}}{d \delta}=\frac{C_{E}^{i} E_{t}^{i} a_{i} p_{i}}{\Delta_{i}}>0
$$

2. Die Abhängigkeit der Steady-State-Variablen vom Relativpreis

Hier muß zuerst untersucht werden, welches Vorzeichen $E_{q}^{i}$ hat

$E_{\mathbf{q}}^{i}{ }^{>}={ }_{<} 0 \Leftrightarrow u_{C C}^{i} C_{E}^{i} C_{\mathrm{q}}^{i}+u_{C}^{i} C_{E \mathbf{q}}^{i}{ }^{>}={ }_{<} 0$

Behauptung: $\quad u_{C C}^{i} C_{E}^{i} C^{i}{ }_{\mathrm{q}}+u_{C}^{i} C_{E \mathbf{q}}^{i}<0$

Ist $C_{q}^{i} \geq 0, d$. h. die Region $i$ ist nicht Ressourcenimporteur, dann ist obige Behauptung erfüllt.

Sei jetzt $C_{q}^{i}<0$, die Region ist Ressourcenimporteur, dann gilt 


$$
u_{C C}^{i} C_{E}^{i} C_{\mathbf{q}}^{i}+u_{C}^{i} C_{E \mathbf{q}}^{i}<0 \Leftrightarrow \frac{u_{C C}^{i}}{u_{C}^{i}}+\frac{C_{E \mathbf{q}}^{i j}}{C_{E}^{i} C_{\mathbf{q}}^{i}}>0 \Leftrightarrow \frac{u_{C C}^{i}}{u_{C}^{i}} C_{i}+\frac{C_{E \mathbf{q}}^{i}}{C_{E}^{i} C_{\mathbf{q}}^{i}} C_{i}>0
$$

Aus den Annahmen über die Wohlfahrtsfunktion folgt

$$
-\frac{u_{C C}^{i}}{u_{C}^{i}} C_{i}<1
$$

Es muß also gezeigt werden, daß

$$
\frac{C_{E \mathbf{q}}^{i}}{C_{E}^{i} C_{\mathbf{q}}^{i}} C_{i} \geq 1
$$

ist.

Betrachten wir zuerst den Fall $E_{i} \in\left[\boldsymbol{E}_{i}, E_{i}{ }^{\max }\right)$

$$
\begin{aligned}
& \frac{C_{E \mathbf{q}}^{i} C_{i}}{C_{E}^{i} C_{\mathrm{q}}^{i}}=\frac{\frac{1}{a_{i}} C_{i}}{\left[\frac{1}{a_{i}}\left(f_{R}^{i}-\mathrm{q}\right)+f_{E}^{i}\right]\left(R_{i}-\mathbf{R}_{i}\right)} \geq 1 \\
& \Leftrightarrow \frac{1}{a_{i}}\left[X_{i}-\mathrm{q}\left(R_{i}-\mathbf{R}_{i}\right)\right] \geq\left(\frac{1}{a_{i}} f_{R}^{i}+f_{E}^{i}\right)\left(R_{i}-\mathbf{R}_{i}\right)-\frac{1}{a_{i}} \mathbf{q}\left(R_{i}-\mathbf{R}_{i}\right) \\
& \Leftrightarrow \frac{1}{a_{i}} X_{i} \geq\left(\frac{1}{a_{i}} f_{R}^{i}+f_{E}\right)\left(R_{i}-\mathbf{R}_{i}\right) \Leftrightarrow \frac{X_{i}}{\left(\frac{1}{a_{i}} f_{R}^{i}+f_{E}^{i}\right) E_{i}} \geq \frac{R_{i}-\mathbf{R}_{i}}{R_{i}} \\
& \Leftrightarrow \frac{X_{i}}{\left(\frac{1}{a_{i}} f_{R}^{i}+f_{E}^{i}\right) E_{i}} \geq \frac{R_{i}-\mathbf{R}_{i}}{R_{i}} \Leftrightarrow\left(\frac{1}{a_{i}} f_{R^{i}}+f_{E}^{i}\right) \frac{E_{i}}{X_{i}} \leq \frac{R_{i}}{R_{i}-\mathbf{R}_{i}}
\end{aligned}
$$

Da die Funktion $f\left(a_{i}^{-1} E_{i}, E_{i}\right)$ konkav ist und für $E_{i}=a_{i} R_{i}=0$ durch den Ursprung geht, gilt 


$$
\left(\frac{1}{a_{i}} f_{R}^{i}+f_{E}^{i}\right) \frac{E_{i}}{X_{i}}<1<\frac{R_{i}}{R_{i}-R_{i}} \text { q.e.d. }
$$

Sei jetzt $E_{i}<E_{i}$ :

$$
\begin{aligned}
& \frac{C_{E \mathbf{q}}^{i} C_{i}}{C_{E}^{i} C_{\mathbf{q}}^{i}}=\frac{\frac{f_{E R}^{i}}{f_{R R}^{i}} C_{i}}{-f_{E}^{i}\left(R_{i}-\mathbf{R}_{i}\right)} \geq 1 \Leftrightarrow \\
& C_{i} \geq-\frac{f_{E}^{i}\left(R_{i}-\mathbf{R}_{i}\right) f_{R R}^{i}}{f_{E R}^{i}}=\frac{X_{i}-\mathbf{q} R_{i}}{R_{i}}\left(R_{i}-\mathbf{R}_{i}\right) \Leftrightarrow \\
& X_{i}-\mathbf{q}\left(R_{i}-\mathbf{R}_{i}\right) \geq \frac{X_{i}}{R_{i}}\left(R_{i}-\mathbf{R}_{i}\right)-\mathbf{q}\left(R_{i}-\mathbf{R}_{i}\right) \Leftrightarrow \mathbf{R}_{i} \geq 0 \text { q.e.d. }
\end{aligned}
$$

Dann ergeben sich die folgenden Abhängigkeiten

$$
\begin{aligned}
& \frac{d E_{i}}{d \mathbf{q}}=\frac{a_{i}\left(\delta+a_{i}\right) E_{\mathbf{q}}^{i}}{\Delta_{i}}<0 \\
& \frac{d S_{i}}{d \mathbf{q}}=\frac{\left(\delta+a_{i}\right) E_{\mathbf{q}}^{i}}{\Delta_{i}}<0 \\
& \frac{d \tau_{i}}{d \mathbf{q}}=-\frac{d p_{i}}{d \mathbf{q}}=\frac{-u_{S S}^{i} E_{\mathbf{q}}^{i}}{\Delta_{i}}<0 \\
& \frac{d C_{i}}{d \mathbf{q}}=\frac{u_{S S}^{i} C_{\mathbf{q}}^{i} E_{\tau}^{i}+a_{i}\left(\delta+a_{i}\right)\left[C_{E}^{i} E_{\mathrm{q}}^{i}++C_{\mathbf{q}}^{i}\right]}{\Delta_{i}} \geq 0
\end{aligned}
$$

15) Dies folgt aus der Homogenität der Produktionsfunktion vom Grade $k<1$ bezüglich $R_{i}$ 
3. Die Auswirkungen der Höhe des Faktorbestandes

Formal ergeben sich hier die gleichen Änderungen wie im autarken Fall des Kapitels 3, jedoch hat $E_{\mathbf{R}}^{i}$ bei Handel ein anderes Vorzeichen:

$$
\begin{aligned}
& \frac{d E_{i}}{d \mathbf{R}_{i}}=\frac{a_{i}\left(\delta+a_{i}\right) E_{\mathbf{R}}^{i}}{\Delta_{i}}<0 \\
& \frac{d S_{t}}{d \mathbf{R}_{i}}=\frac{\left(\delta+a_{i}\right) E_{\mathbf{R}}^{i}}{\Delta_{i}}<0 \\
& \frac{d \tau_{i}}{d \mathbf{R}_{i}}=-\frac{d p_{i}}{d \mathbf{R}_{i}}=\frac{-u_{S S}^{i} E_{\mathbf{R}}^{i}}{\Delta}<0 \\
& \frac{d C_{i}}{d \mathbf{R}_{i}}=\frac{\mathbf{q} E_{\tau}^{i}\left[a_{i}\left(\delta+a_{i}\right) u_{C}^{i} C_{E E}^{i}+u_{S S}^{i}\right]}{\Delta_{i}}>0
\end{aligned}
$$




\section{Kapitel 6: Interregionale Verflechtung durch Diffusion und}

\section{Handel}

\section{PROBLEMSTELLUNG}

In diesem Teil der Arbeit werden die Modelle von Kapitel 4 und Kapitel 5 zusammengefaßt. Es werden dynamische Modelle vorgestellt, die explizit Handelsbeziehungen berücksichtigen und den Schadstoffexport über Grenzen hinweg unter Einbeziehung der Schadstoffakkumulation diskutieren. In Schaubild 6.1 ist die Struktur der Ökonomie dargestellt.

In Analogie zu Kapitel 5 wird der Fall untersucht, daß die betrachtete Ökonomie ein kleiner Bestandteil eines größeren Gebildes ist, die Preise auf den Märkten werden von der Ökonomie nicht beeinflußt.

Die so definierte Ökonomie wird wiederum unter den in Kapitel 4 durchgespielten institutionellen Arrangements betrachtet: Autonome Regionalregierungen, Zentralregierung und autonome Regionalregierungen, die von der Zentralregierung vorgegebene Restriktionen beachten müssen.

Es gelten die folgenden Spezifizierungen der Ökozustand-Funktionen [vgl. die Gleichungen (4.1.i) und (4.1.ii) sowie (4.2.i) und (4.2.ii)]:

(i)

$$
\frac{d S_{1}}{d t}=E_{1}+\mathrm{Y}_{2} E_{2}-\mathrm{a}_{1} S_{1}
$$

(ii)

$$
\frac{d S_{2}}{d t}=\left(1-\mathrm{Y}_{2}\right) E_{2}-\mathrm{a}_{2} S_{2}
$$

woraus sich folgende Stationaritätsbedingungen ergeben

$$
S_{1}=\frac{1}{a_{1}}\left[E_{1}+\mathrm{Y}_{2} E_{2}\right]
$$

$$
S_{2}=\frac{1-\mathrm{Y}_{2}}{\mathrm{a}_{2}} E_{2}
$$

Es werden wiederum die unterschiedlichen institutionellen Arrangements unter der Prämisse untersucht, daß die betrachtete Ökonomie ein kleines 


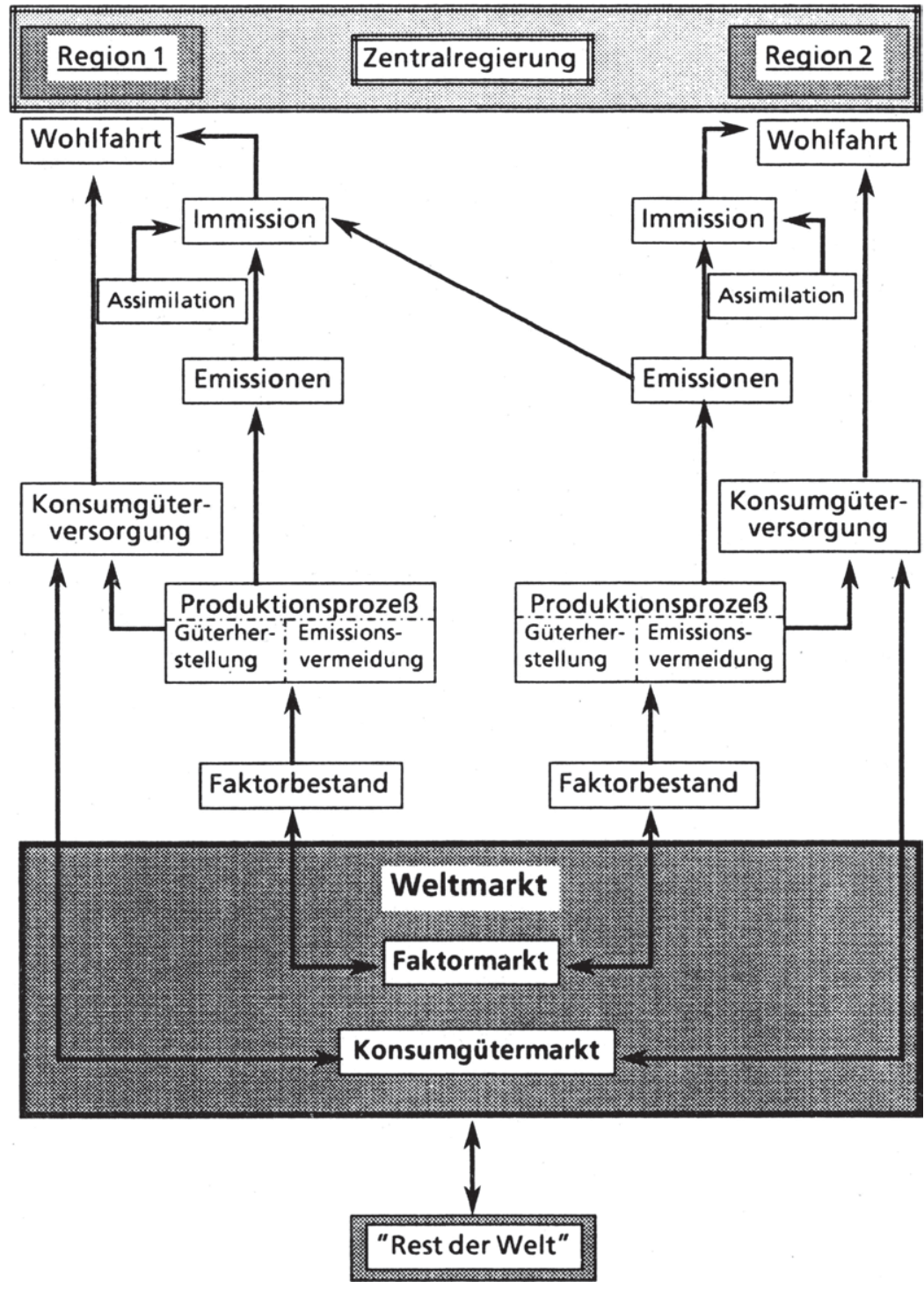

Schaubild 6.1: Die Struktur der Ökonomie 
Land ist, d. h. der Weltmarktrelativpreis konstant vorgegeben ist:

(6.3) $\quad q(t)=\frac{q_{R}(t)}{q_{X}(t)}=q=$ konstant $\quad q_{X}(t)>0 \forall t \in[0, \infty)$.

Daher gilt die Zahlungsbilanzrestriktion [vgl. Gleichung (3.8')]

(6.4) $\quad C_{i}-X_{i}+q\left(R_{i}-R_{i}\right)=0$ 


\section{AUTONOME REGIONALBEHÖRDEN}

\subsection{DIE OPTIMIERUNGSPROBLEME UND DIE OPTIMALBEDINGUNGEN ${ }^{1)}$}

In der hier vorgenommenen Analyse wird davon ausgegangen, daß die Regionen autonome Regionalregierungen haben, die die Wohlfahrt ihrer jeweiligen Region maximieren, ohne die Auswirkungen auf die andere Region zu beachten. Für Region 2 ergibt sich das folgende intertemporale Maximierungsproblem:

$$
\begin{aligned}
& \operatorname{Max} W_{2}=\int_{0}^{\infty} u^{2}\left(C_{2}, S_{2}\right) e^{-\delta t} d t \\
& \text { u.d.B. (3.1) } \quad f^{2}\left(R_{2}, E_{2}\right)-X_{2} \geq 0 \\
& \text { (3.2) } \quad a_{2} R_{2}-E_{2} \geq 0 \\
& \text { (6.3) } \quad C_{2}-X_{2}+q\left(R_{2}-R_{2}\right)=0 \\
& \text { (6.1.ii) } \frac{d S_{2}}{d t}=\left(1-Y_{2}\right) E_{2}-a_{2} S_{2} \\
& X_{2}, C_{2}, R_{2}, E_{2} \geq 0 \forall t \in[0, \infty) \\
& S_{2}(0)=S_{2}{ }^{0} \text { gegeben } \\
& \delta \text { gegeben und positiv }
\end{aligned}
$$

Für Region 1 ergibt sich das analoge Problem:

$$
\begin{aligned}
& \operatorname{Max} W_{1}=\int_{0}^{\infty} u^{1}\left(C_{1}, S_{1}\right) e^{-\delta t} d t \\
& \text { u.d.B. (3.1) } \quad f^{1}\left(R_{1}, E_{1}\right)-X_{1} \geq 0 \\
& \text { (3.2) } \quad a_{1} R_{1}-E_{1} \geq 0 \\
& \text { (6.3) } C_{1}-X_{1}+\mathbf{q}\left(R_{1}-\mathbf{R}_{1}\right)=0 \\
& \text { (6.1.i) } \frac{d S_{1}}{d t}=E_{1}+Y_{2} E_{2}-a_{1} S_{1}
\end{aligned}
$$

1) Vgl. hierzu das analoge Problem des Kapitels 4, Abschnitt 2. 


$$
\begin{aligned}
& X_{1}, C_{1}, R_{1}, E_{1} \geq 0 \forall t \in[0, \infty) \\
& S_{1}(0)=S_{1}^{0} \text { gegeben }
\end{aligned}
$$

$\delta$ gegeben und positiv

$$
E_{2}=E_{2}^{\infty} \text { gegeben }
$$

Dies führt zu den Lagrange-Funktionen in laufenden Werten $(i, j=1,2$; $i \neq j ; \gamma_{1}=0, \gamma_{2}>0$ ):

$$
\begin{aligned}
L^{i}=u^{i}\left(C_{i}, S_{i}\right)+ & p_{i}\left[\left(1-Y_{i}\right) E_{i}+\gamma_{j} E_{j}-\alpha_{i} S_{i}\right] \\
& +\lambda^{i}{ }_{1}\left[f\left(R_{i}, E_{i}\right)-X_{i}\right]+\lambda^{i}{ }_{2}\left[a_{i} R_{i}-E_{i}\right] \\
& +\lambda^{i}{ }_{3} C_{i}+\lambda^{i}{ }_{4} R_{i}+\lambda^{i}{ }_{5} E_{i}+\lambda^{i}{ }_{6} X_{i} \\
& +\mu^{i}\left[C_{i}-X_{i}+\mathbf{q}\left(R_{i}-R_{i}\right)\right]
\end{aligned}
$$

Damit erhält man folgende Optimalbedingungen:

(i)

$$
L_{C}^{i}=u_{C}^{i}+\lambda_{3}^{i}+\mu^{i}=0
$$

(ii)

$$
L^{i}{ }_{X}=-\lambda^{i}{ }_{1}+\lambda_{6}^{i}-\mu^{i}=0
$$$$
x_{i} \geq 0
$$

$$
L_{R}^{i}=\lambda^{i}{ }_{1} \dot{f}_{R}+\lambda^{i}{ }_{2} a_{i}+\lambda^{i}{ }_{5}+\mathrm{q} \mu^{i}=0
$$$$
\boldsymbol{R}_{i} \geq 0
$$

(iv.1)

$$
L_{E}^{1}=p_{1}+\lambda^{1}{ }_{1} f_{E}{ }_{E}-\lambda^{1}{ }_{2}+\lambda^{1}{ }_{5}=0
$$

$$
\begin{aligned}
& L_{p}^{1}=\frac{d S_{1}}{d t}=E_{1}+Y_{2} E_{2}-a_{1} S_{1} \\
& L_{p}^{2}=\frac{d S_{2}}{d t}=\left(1-Y_{2}\right) E_{2}-a_{2} S_{2}
\end{aligned}
$$

(vi)

$$
L_{S}^{i}=-\frac{d p_{i}}{d t}+\delta p_{i}=u_{S}^{i}-a_{i} p_{i}
$$

(6.8)

(vii)

$$
\lambda^{i}{ }_{1}\left[f\left(R_{i}, E_{i}\right)-X_{i}\right]=0
$$

(viii)

$$
\lambda^{i}{ }_{2}\left[a_{i} R_{i}-E_{i}\right]=0
$$


(xi)

(xii)

(xiii)

$$
\begin{array}{ll}
\lambda_{5}^{i} E_{i}=0 & \lambda_{5}^{i} \geq 0 \\
\lambda_{6}{ }_{6}{ }_{6}{ }_{i}=0 & \lambda^{i}{ }_{6} \geq 0
\end{array}
$$

$$
\mu^{i}\left[C_{i}-X_{i}+\mathbf{q}\left(R_{i}-\mathbf{R}_{i}\right)\right]=0
$$

Die Diskussion der Optimalbedingungen ist die gleiche wie in Kapitel 5, Abschnitt 2, so daß an dieser Stelle auf die Gleichungen (5.9), (5.10), (5.12), (5.13) und (5.14) verwiesen werden kann, die auch für Kapitel 6 gelten.

Für die Beziehung zwischen optimaler Steuer und dem Schattenpreis ergibt sich jedoch eine Änderung dadurch, daß die Diffusionsbeziehungen berücksichtigt werden. Für Region 1 ergibt sich die analoge Beziehung wie Gleichung (5.15):

$$
\tau_{1}= \begin{cases}\tau_{1} & \text { für } p_{1} \geq p_{1} ; \\ -p_{1} & \text { für } p_{1} \in\left(p_{1} ; 0\right) \\ 0 & \text { für } p_{1}=0\end{cases}
$$

Für Region 2 ergibt sich folgende Beziehung:

$$
\tau_{2}= \begin{cases}\tau_{2}{ }^{\prime} & \text { für } p_{2} \leq p_{2}^{\prime}, \quad p_{2}^{\prime} \equiv \frac{-\tau_{2}{ }^{\prime}}{1-\gamma_{2}} \\ -\left(1-\gamma_{2}\right) p_{2} & \text { für } p_{2} \in\left(p_{2}^{\prime}, 0\right) \\ 0 & \text { für } p_{2}=0\end{cases}
$$

In Analogie zu Gleichung (4.11) wird bei Bedingung (6.10) berücksichtigt, da $\beta$ nur das $\left(1-\gamma_{2}\right)$-fache einer Emissionseinheit in Region 2 verbleibt, so daß die Steuer nur das $\left(1-\gamma_{2}\right)$-fache der intertemporalen Wohlfahrtsänderung durch eine Emissionseinheit ist.

Für bis auf die Diffusionsbeziehungen identische Regionen sind die Beziehungen zwischen Emissionen, Schattenpreis und Steuern in Schaubild 6.2 
zusammengefaßt [vgl. hierzu auch die Schaubilder 4.2 und 5.4].

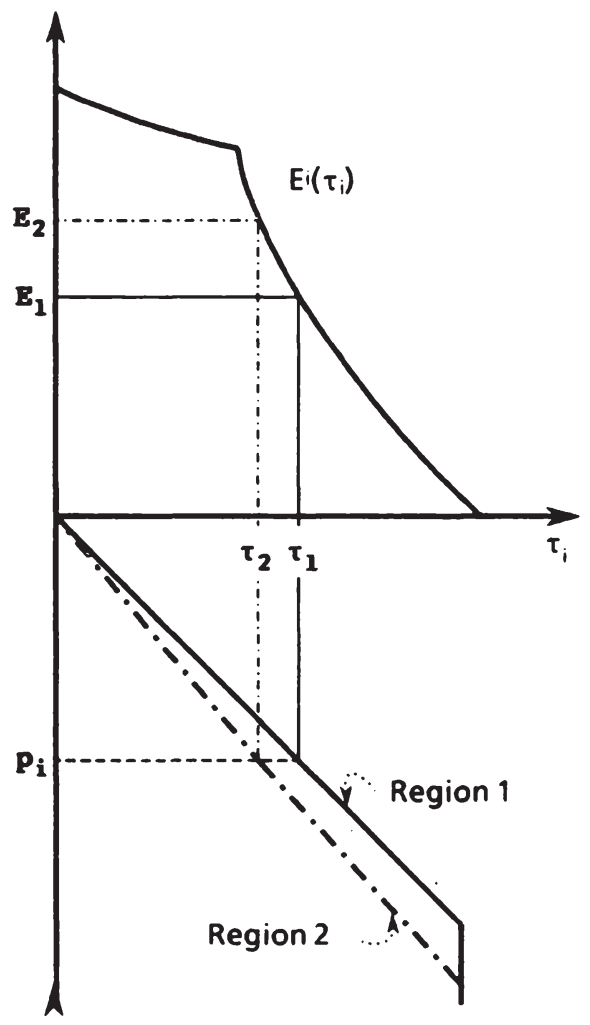

Schaubild 6.2: Die Beziehungen zwischen Emissionen, Steuer und Zukunftsschaden 


\subsection{DAS STEADY-STATE DER ÖKONOMIE}

\subsubsection{Die Ableitung für Region 2}

Die Ableitung erfolgt analog zu der in Kapitel 5 gegebenen, unter Berücksichtigung, daß nur das $\left(1-\gamma_{2}\right)$-fache der Emissionen in Region 2 verbleibt, so da $\beta$ hier auf eine Darstellung verzichtet werden kann [vgl. auch Kapitel 4, Abschnitt 2.2].

\subsubsection{Das Steady-State für Region 1}

Für Region 1 kommt eine Abhängigkeit von Region 2 hinzu, da sie einen Teil der Emissionen dieser Region in ihr Ökosystem aufnehmen muß. Da jedoch die Regionalregierung von Region 1 annahmegemäß die SteadyState-Emissionen aus Region 2 kennt und nur Steady-States betrachtet werden, kann das Differentialgleichungssystem

(i)

$$
\frac{d S_{1}}{d t}=E^{1}\left(-p_{1}\right)+Y_{2} E_{2}-a_{1} S_{1}
$$

(ii)

$$
\frac{d p_{1}}{d t}=\left(\delta+a_{1}\right)-u_{S}^{1}
$$

für ein gegebenes $S_{1}{ }^{0}$ und ein geeignet gewähltes $p_{1}(0)$ eindeutig gelöst werden und man erhält eine stationäre Lösung $\left(p_{1}{ }^{\infty}, S_{1}{ }^{\infty}\right)$, die jedoch abhängig ist von den Emissionen aus Region 2. Je größer $E_{2}{ }^{\infty}$ ist, desto niedriger ist $p_{1}^{\infty}$ und desto höher ist $S_{1}^{\infty}$. In Schaubild 6.3 sind die Anpassungen der Region 1 dargestellt ${ }^{2)}$.

Je höher der Emissionsimport aus Region 2 ist, desto weiter nach außen verschiebt sich die stationäre Ökozustand-Funktion der Region 1 (Quadrant II). D. h. aber, daß auch die Funktion $d S_{1} / d t=0$ keinen festen Verlauf mehr hat, sondern je größer $E_{2}{ }^{\infty}$ ist, desto weiter außen verläuft diese Funktion (Quadrant V).

Je größer die importierten Emissionen aus Region 2 sind, desto weniger

2) Vgl. hierzu die analogen Ausführungen in Kapitel 4, Abschnitt 2.3. 


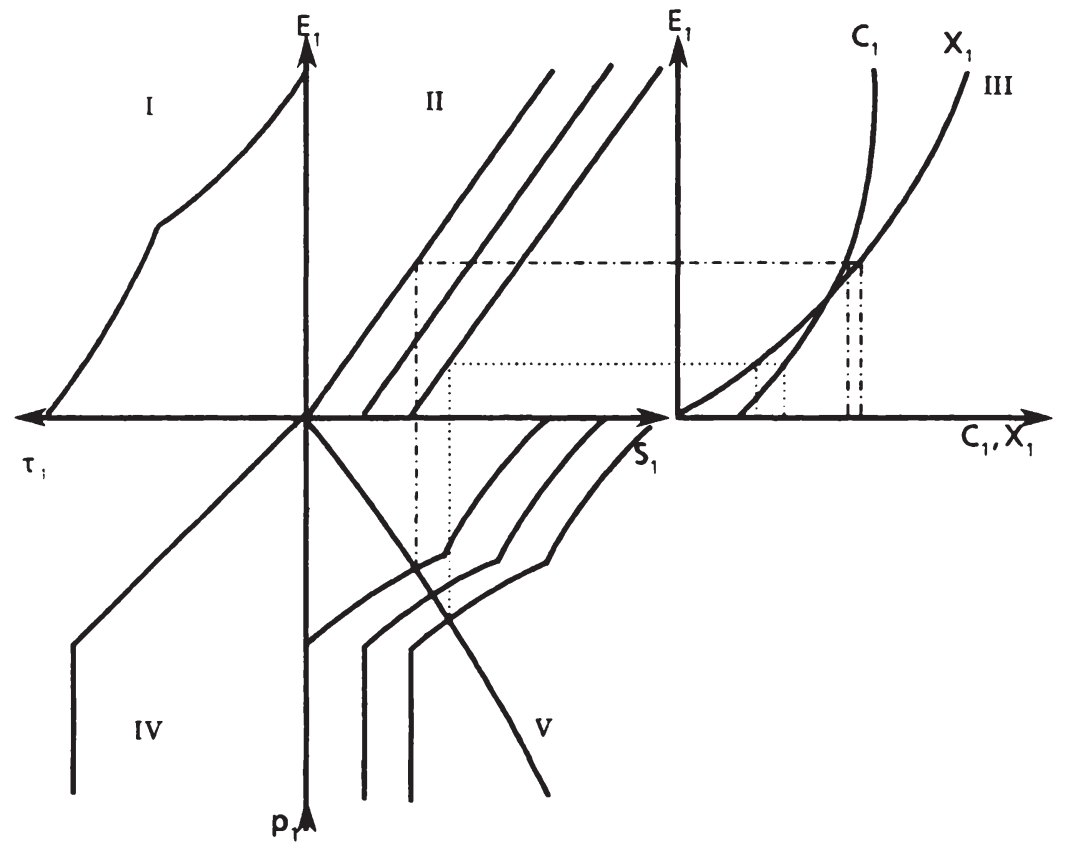

Schaubild 6.3: Die Anpassungen der Region 1

wird in Region 1 produziert, desto wahrscheinlicher ist es, daß die Region Faktoren exportiert, um fertige Konsumgüter zu importieren, da so die Eigenverschmutzung relativ niedrig wird und damit ein angestrebtes Umweltziel erreicht werden kann. 
Ein Gesamt-Steady-State der Ökonomie ist dann erreicht, wenn alle Werte der Variablen in der Ökonomie stationär sind:

$$
\frac{d S_{1}}{d t}=\frac{d p_{1}}{d t}=\frac{d S_{2}}{d t}=\frac{d p_{2}}{d t}=0
$$

Für Region 1 kann man Steady-State Werte der Variablen in Abhängigkeit alternativer Gleichgewichtswerte der Emissionen aus Region 2 errechnen [vgl. Kapitel 4, Abschnitt 2.4.1]:

$$
\begin{aligned}
E_{1}-E^{1}\left(\tau_{1}\right) & =0 \\
\tau_{1}+p_{1} & =0 \\
E_{1}-\alpha_{1} S_{1} & =-\gamma_{2} E_{2} \\
\left(\delta+\alpha_{1}\right) p_{1}-u_{S}^{1} & =0
\end{aligned}
$$

Totale Differentiation nach $E_{2}$ ergibt folgendes Gleichungssystem $\left(E_{1} \neq\right.$ $\left.E_{1}\right)$

(6.12) $\left[\begin{array}{cccc}1 & -\alpha_{1} & 0 & 0 \\ 0 & -u_{S S}^{1} & \delta+\alpha_{1} & 0 \\ 0 & 0 & 1 & 1 \\ 1 & 0 & 0 & -E^{1} \tau\end{array}\right]\left[\begin{array}{l}\frac{d E_{1}{ }^{*}}{d E_{2}} \\ \frac{d S_{1}^{*}}{d E_{2}} \\ \frac{d p_{1}^{*}}{d E_{2}} \\ \frac{d \tau_{1}^{*}}{d E_{2}}\end{array}\right]=\left[\begin{array}{c}-\gamma_{2} \\ 0 \\ 0 \\ 0\end{array}\right]$

Die Systemdeterminante ist

$$
\Delta_{1}=u_{S S^{1}}^{E^{1}}+\alpha_{1}\left(\delta+\alpha_{1}\right)
$$


und man erhält für parametrische Änderungen des Steady-State-Wertes der Emissionen aus Region 2 folgende Änderungen der Steady-State-Werte in Region 1:

$$
\begin{aligned}
& \frac{d E_{1}^{*}}{d E_{2}}=\frac{-\mathrm{Y}_{2} u_{S S}^{1} E_{\mathrm{r}}^{1}}{\Delta_{1}}<0 \\
& \frac{d S_{1}^{*}}{d E_{2}}=\frac{\mathrm{Y}_{2}\left(\delta+\mathrm{a}_{1}\right)}{\Delta_{1}}>0 \\
& \frac{d p_{1}^{*}}{d E_{2}}=\frac{\mathrm{Y}_{2} u_{S S}^{1}}{\Delta_{1}}<0 \\
& \frac{d \tau_{1}^{*}}{d E_{2}}=-\frac{\mathrm{Y}_{2} u_{S S}^{1}}{\Delta_{1}}>0
\end{aligned}
$$

Die Steady-State-Werte der Emissionen in Region $1 E_{1}{ }^{*}$ sinken für steigendes $E_{2}$, während die Steuern $\tau_{1}{ }^{*}$ und die Immissionen $S_{1}{ }^{*}$ steigen.

Betrachten wir jetzt die Funktion

$$
E_{1}^{*}=\varepsilon^{1}\left(E_{2}\right), \quad \varepsilon_{E}^{1} \in\left(-\gamma_{2}, 0\right)
$$

Ein Gesamtgleichgewicht der Ökonomie ist dann erreicht, wenn gilt

$$
E_{1}^{\infty}=\varepsilon^{1}\left(E_{2}^{\infty}\right.
$$

Graphisch läßt sich das Gesamtgleichgewicht wie in Schaubild 6.4 dargestellt ableiten.

Da die Emissionen in Region 2 unabhängig sind von den Emissionen in Region 1, ist der Steady-State-Wert als Vertikale in Schaubild 6.4 eingezeichnet. 


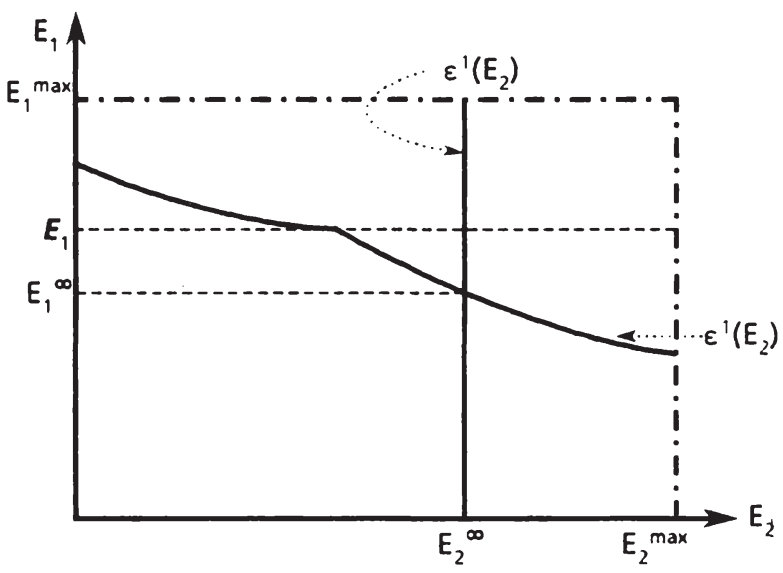

Schaubild 6.4: Die Ableitung des Gleichgewichts

Die $\varepsilon^{1}\left(E_{2}\right)$-Funktion hat einen fallenden Verlauf. In $E_{1}{ }^{*}=E_{1}$ hat sie einen Knick.

Im Schnittpunkt von $E_{2}{ }^{\infty}$ mit $\varepsilon^{1}\left(E_{2}{ }^{\infty}\right)$ liegt das Gesamt-Steady-State der Ökonomie. 


\subsection{Die EigenSCHAFTEN DES GLEICHGEWICHTS}

Es wird jetzt unterstellt, daß sich die Gesamtökonomie in einem Gleichgewicht befindet und es wird untersucht, welche Auswirkungen die Größe der Parameter auf das stationäre Gleichgewicht haben. Im Anhang zu diesem Kapitel wird die Ableitung der Ergebnisse dargestellt. Hier werden die Änderungsrichtungen in folgender Tabelle zusammengefaßt:

\begin{tabular}{|c|c|c|c|c|c|c|c|c|c|c|}
\hline & $d E_{1}$ & $d S_{1}$ & $d p_{1}$ & $d \tau_{1}$ & $d C_{1}$ & $d E_{2}$ & $d S_{2}$ & $d p_{2}$ & $d \tau_{2}$ & $d C_{2}$ \\
\hline$d \delta$ & $?$ & $\oplus$ & $?$ & $?$ & $?$ & $\oplus$ & $\oplus$ & $\oplus$ & $\theta$ & $\oplus$ \\
\hline$d \alpha_{1}$ & $\oplus$ & $?$ & $\oplus$ & $\theta$ & $\oplus$ & - & $\bullet$ & $\bullet$ & $\bullet$ & $\bullet$ \\
\hline$d \mathbf{R}_{1}$ & $\theta$ & $\theta$ & $\oplus$ & $\theta$ & $\oplus$ & $\bullet$ & • & - & - & - \\
\hline$d \alpha_{2}$ & $\theta$ & $\oplus$ & $\theta$ & $\oplus$ & $\theta$ & $\oplus$ & $?$ & $\oplus$ & $\theta$ & $\oplus$ \\
\hline$d \mathbf{R}_{2}$ & $\oplus$ & $\theta$ & $\oplus$ & $\theta$ & $\oplus$ & $\theta$ & $\theta$ & $\oplus$ & $\theta$ & $\oplus$ \\
\hline$d \mathbf{q}$ & $?$ & $\theta$ & $\oplus$ & $\theta$ & $?$ & $\theta$ & $\theta$ & $\oplus$ & $\theta$ & $?$ \\
\hline$d \gamma_{2}$ & $\theta$ & $\oplus$ & $\theta$ & $\oplus$ & $\theta$ & $\oplus$ & $?$ & $?$ & $\theta$ & $\oplus$ \\
\hline
\end{tabular}

- keine Auswirkungen

Tabelle 6.1: Die Änderungen des Steady-States in Abhängigkeit der Größe der Parameter

\subsubsection{Die Auswirkungen der Höhe der Diskontrate}

Die Höhe der Diskontrate hat auf die Steady-State-Werte der Region 2 eindeutige Auswirkungen. Je größer die Diskontrate ist, desto höher sind die Emissionen, die Immissionen und der Konsum, während die Steuer und der bewertete Zukunftsschaden umso niedriger sind. Für Region 1 erhält man nur ein eindeutiges Vorzeichen für die Immissionen: sie steigen, je größer $\delta$ ist. Die Wirkungen auf die anderen Steady-State-Werte in Region 1 sind nicht eindeutig. Hier ergeben sich die analogen Effekte' wie im autarken Fall [vgl. Bedingung (4.23) und die Diskussion dazu in Kapitel 4, Abschnitt 2.4.2.1].

Die Auswirkungen der Höhe der Diskontrate auf das Steady-State wird also durch die Diffusionsbeziehungen entscheidend bestimmt, während der Einfluß der Handelsbeziehungen nur schwach ins Gewicht fällt. 


\subsubsection{Die Auswirkungen der Größe der Faktorausstattungen}

Die Auswirkungen der Höhe der Faktorausstattung auf das Steady-State sind analog zu den in Kapitel 5 diskutierten Auswirkungen [vgl. Abschnitt 2.4.2], wobei jedoch noch die Effekte für Region 1 hinzukommen, die sich aus der Abhängigkeit durch die Diffusionsbeziehungen der Region 1 von Region 2 ergeben. Konträr zu dem in Kapitel 4 [Abschnitt 2.4.2.3] diskutierten Fall von autarken Regionen, ist es für die emissionsimportierende Region 1 vorteilhaft, wenn Region 2, die emissionsexportierende Region, reich mit Produktionsfaktoren ausgestattet ist, da, je reicher die Region 2 ist, dort umso weniger produziert wird und damit umso weniger Emissionen von Region 2 nach Region 1 exportiert werden. Je größer der Faktorbestand in Region 2 ist, umso mehr kann in Region 1 produziert werden (d. h. emittiert) und umso größer ist der Konsum in Region 1. Die Immissionen dagegen sind in Region 1 c. p. umso niedriger, je höher der Faktorbestand in Region 2 ist.

Je größer der Faktorbestand c. p. in Region 2 ist, desto höher ist die Steady-State Wohlfahrt in Region 1 und in Region 2 und damit in der Gesamtökonomie.

\subsubsection{Die Auswirkungen der Höhe der Assimilationskoeffizienten}

Auch die Auswirkungen der Größe der Assimilationskoeffizienten auf das Steady-State sind hauptsächlich durch die Diffusionsbeziehungen bestimmt, so daß wiederum auf Abschnitt 2.4.2.2 in Kapitel 4 und Abschnitt 2.3 in Kapitel 3 verwiesen werden kann.

Die Handelsbeziehungen der Regionen sind bei unterschiedlichen Assimilationskoeffizienten nicht entscheidend für die Steady-State Variablen, sondern die Auswirkungen, die sich über das Ökosystem ergeben, sind ausschlaggebend.

\subsubsection{Die Auswirkungen der Höhe des Relativpreises}

Eine exogene Erhöhung des Relativpreises q bedeutet, daß der Faktor relativ zum Konsumgut teurer wird. Diese Preiserhöhung kann sich durch 
eine Nachfrageverschiebung auf dem Weltmarkt ergeben, was in dieser Analyse jedoch von zweitrangiger Bedeutung ist.

Bei steigendem Relativpreis q sinken die Steady-State Immissionen in Region 1 und der Zukunftsschaden sinkt ebenfalls (d. h. die Steuer sinkt), während für die Emissionen und den Konsum keine eindeutige Aussage gemacht werden kann. In Region $2^{3}$ sinken die Emissionen, die Immissionen und der Zukunftsschaden, was impliziert, daß auch die Steuer sinkt, während für den Konsum wiederum keine eindeutige Aussage gemacht werden kann.

Betrachten wir zuerst die Änderung des Konsums in Region 2 bei steigendem Relativpreis:

Ist Region 2 in der Ausgangslage Ressourcenimporteur, d. h. $C^{2}{ }_{q}=$ $-\left(R_{2}-R_{2}\right)<0$, dann ist die Änderung des Konsums bei steigendem Relativpreis eindeutig: der Konsum sinkt. Durch die Verteuerung des Faktors muß bei konstanter Produktion mehr Konsumgut exportiert werden, damit die Faktormenge konstant bleibt (dies folgt aus der Zahlungsbilanzrestriktion (6.3)), bzw. bei konstanten Importwert $\mathrm{q} R_{2}$ sinkt die Produktion, da weniger Faktoren zur Verfügung stehen.

Ist Region 2 in der Ausgangslage Ressourcenexporteur, ist die Auswirkung des steigenden Relativpreises nicht mehr eindeutig; dann gilt:

$$
\frac{d C_{2}}{d \mathbf{q}} \geq_{0} \Leftrightarrow\left(1-\mathrm{\gamma}_{2}\right) \frac{\left(1-\mathrm{\gamma}_{2}\right) u_{S S}^{2}}{\mathrm{a}_{2}\left(\delta+\mathrm{a}_{2}\right)} \frac{C_{\mathrm{q}}^{2}}{C_{E}^{2}}>-u_{C}^{2}\left(\frac{C_{E E}^{2} C_{\mathrm{q}}^{2}}{C_{E}^{2}}-C_{E \mathbf{q}}^{2}\right)
$$

Dies ist äquivalent zu

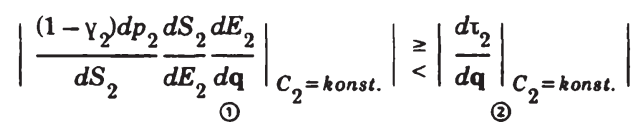

Es wird also die Größe zweier Effekte betrachtet, die für konstantes $C_{2}$ auf die Steuer $\tau_{2}$ wirken durch erhöhtes q. Effekt (1) gibt die Änderung der

3) Vgl. die äquivalenten Aussagen in Kapitel 5. 
Steuer für konstanten Konsum an, die sich über die Änderung des Schattenpreises und damit der Immissionen ergibt, während Effekt (2) die Änderung der Steuer über die Änderung der Emissionen für konstantes $C_{2}$ angibt.

Ist der Faktorbestand in Region 2 genügend groß bzw. die Umweltpolitik sehr restriktiv, dann steigt durch den steigenden Relativpreis auf dem Weltmarkt der Gleichgewichtskonsum in dieser Region. Dieser Fall ist in Schaubild 6.5 dargestellt. Daß Region 2 für steigenden Konsum Ressour-

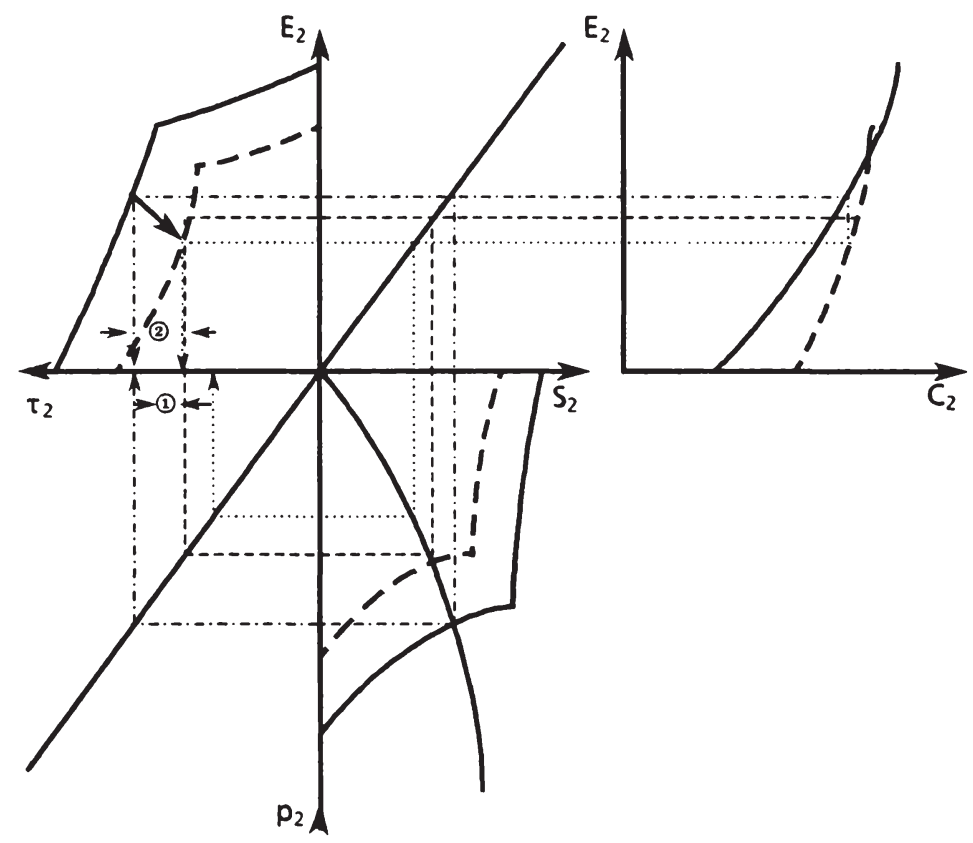

Schaubild 6.5: Die Änderung des Konsums für erhöhtes q in Region 2 cenexporteur ist, ist eine notwendige, aber nicht hinreichende Bedingung. Ohne Umweltpolitik wäre die Bedingung hinreichend, jedoch kann bei Umweltpolitik der Wohlfahrtseffekt durch die Preissteigerung der Ressource überkompensiert werden durch den Wohlfahrtseffekt durch die Verminderung der Immissionen, so daß es sich für die Region lohnen 
kann, weniger zu konsumieren und damit weniger zu emittieren und damit die Immissionen stärker zu senken [vgl. hierzu die äquivalente Ableitung in Kapitel 5].

Betrachten wir jetzt Region 1 bei steigendem Relativpreis q. Wie aus Tabelle 6.1 ersichtlich, ist der Effekt auf die Emissionen nicht eindeutig. Es gilt

$$
\frac{d E_{1}}{d \mathbf{q}} \geq_{0} \Leftrightarrow-\frac{u_{S S}^{1}}{a_{1}\left(\delta+a_{1}\right)} Y_{2} E_{\mathbf{q}}^{* 2}>u_{C}^{1} C_{E \mathbf{q}}^{1}+u_{C C}^{1} C_{E}^{1} C_{\mathbf{q}}^{1}
$$

Dies ist äquivalent zu

$$
\left.\left|\frac{d \tau_{1}}{d S_{1}} \frac{d S_{1}}{d E_{2}}\right|_{E_{1}=\text { konst. }} \frac{d E_{2}}{d \mathbf{q}}|\geq| \frac{d \tau_{1}}{d \mathbf{q}}\right|_{E_{1}=\text { konst. }} \mid
$$

Es sind wieder zwei Effekte zu betrachten: Effekt (1) gibt die Steueränderung für sinkendes $E_{2}\left(d E_{2} / d q<0\right)$ an, also den Effekt, der durch die Diffusion von Region 2 auf Region 1 wirkt, während Effekt (2) die direkte Steueränderung durch die Vergrößerung des Relativpreises angibt. In Schaubild 6.6 sind die Effekte graphisch dargestellt.

Effekt (1) ist umso größer, je kleiner die Emissionen in Region 1 in der Ausgangslage sind, d. h. je restriktiver die Umweltpolitik in dieser Region ist bzw. je kleiner die Assimilationsfähigkeit des Ökosystems ist, denn dieser Effekt hängt entscheidend von der Steigung der $d p_{1} / d t=0$-Kurve ab. Je steiler diese ist, desto größer ist der Diffusionseffekt (1). Effekt (3) der direkte Relativpreiseffekt, gibt an, wie sich die Steuer ändert bei konstanten Emissionen in Region 1, wenn sich der Relativpreis ändert.

Zum Abschluß untersuchen wir die Konsumänderung in Region 1 bei einer Relativpreiserhöhung:

$$
\frac{d C_{1}}{d q} \geq_{0} \Leftrightarrow u_{S S}^{1} C_{\mathrm{q}}^{1}+a_{1}\left(\delta+a_{1}\right) u_{C}^{1}\left|C_{E E}^{1} C_{q}^{1}-C_{E q}^{1} C_{E}^{1}\right| \leq u_{S S}^{1} C_{E}^{1} \mathrm{Y}_{2} E_{\mathrm{q}}^{* 2}
$$




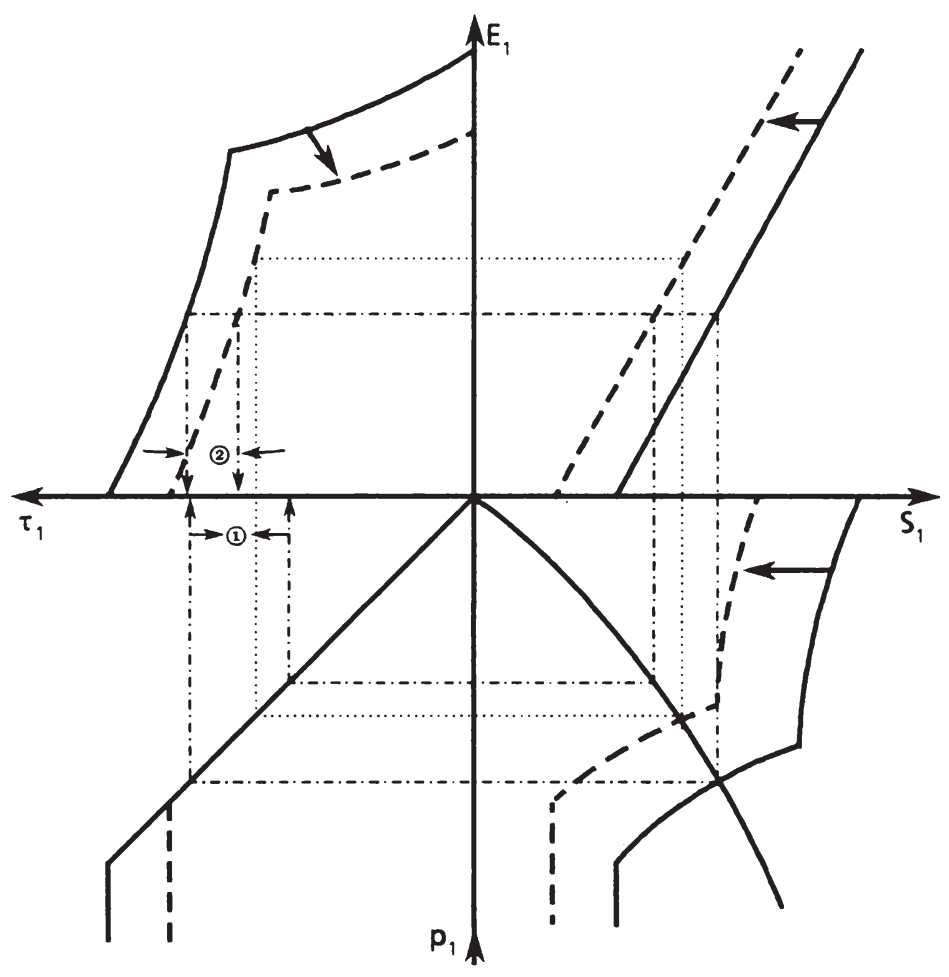

Schaubild 6.6: Emissionsänderung in Region 1 bei steigendem Relativpreis

Dies ergibt

$$
\left.\left|\frac{d \tau_{1}}{d S_{1}}\right| \frac{d S_{1}}{d E_{2}}\right|_{E_{1}=\text { konst. }} \frac{d E_{2}}{d \mathbf{q}}+\left.\left.\frac{d S_{1}}{d E_{1}}\right|_{E_{2}=\text { konst. }} \frac{d E_{1}}{d q}\right|_{C_{1}=\text { konst }}|| \geq\left|\frac{d \tau_{1}}{d q}\right|_{C_{1}=\text { konst. }} \mid
$$

In Analogie zu Region 2 gibt Effekt (1) die Änderung der Steuer für konstanten Konsum an, die sich über die Änderung der Immissionen ergibt, während Effekt (2) die Änderung der Steuer über die Änderung der Emissionen in Region 1 für konstantes $C_{1}$ angibt. Der Immissionseffekt (1) wird durch die Emissionssenkung in Region 2 verstärkt. Für Region 1 ist es we- 
der hinreichend noch notwendig, daß sie Ressourcenexporteur sein muß, um den Konsum zu steigern. Ist die Emissionssenkung in Region 2 durch einen größeren Relativpreis genügend groß, kann es sein, daß Region 1 Ressourcenimporteur ist und der Gleichgewichtskonsum in Region 1 steigt. Dieser Fall ist in Schaubild 6.7 dargestellt. Andererseits kann es

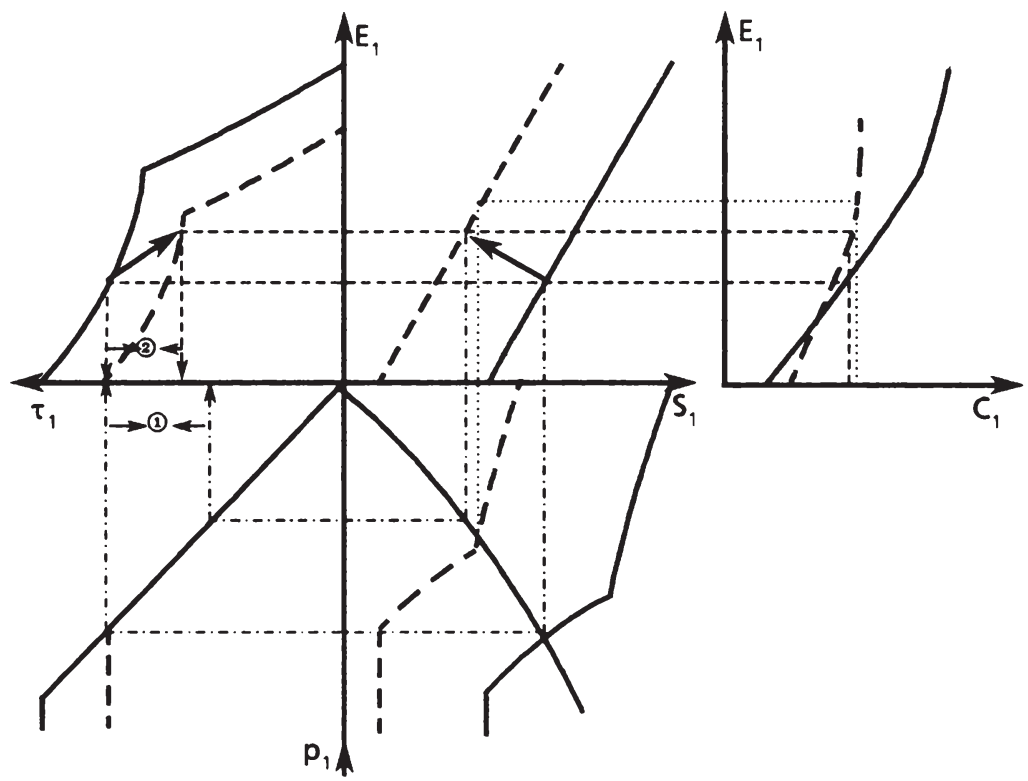

Schaubild 6.7: Die Konsumänderung in Region 1 bei steigendem $q$

sein, wenn die Emissionsminderung in Region 2 genügend klein ist, daß trotz Ressourcenexports der Konsum in Region 1 sinkt.

Eine exogene Relativpreissteigerung senkt die Immissionen in der Ökonomie, d. h. sowohl in Region 1 als auch in Region $2 \operatorname{sinken~} S_{1}{ }^{\infty}$ bzw. $S_{2}{ }^{\infty}$. Den entscheidenden Beitrag dazu liefert die Senkung der Emissionen in Region 2. Ist diese Senkung genügend groß, dann können die Emissionen in Region 1 trotz der Immissionssenkung steigen. Über die Änderung der Konsummenge kann keine eindeutige Aussage gemacht werden. Sind die Regionen beide Ressourcenexporteure und ist der Ressourcenbestand genügend groß, dann steigt der Konsum. In diesem Fall ist eine eindeutige 
Wohlfahrtsverbesserung der Ökonomie durch den steigenden Relativpreis gegeben. Sinkt der Konsum, dann ist die Wohlfahrtsänderung abhängig vom Ausmaß der Effekte. Durch die Immissionssenkung hat man einen positiven Wohlfahrtseffekt, der jedoch durch den negativen Wohlfahrtseffekt durch den verminderten Konsum konterkariert wird.

\subsubsection{Die Auswirkungen der Höhe des Diffusionskoeffizienten}

Eine Erhöhung des Diffusionskoeffizienten kann dahingehend interpretiert werden, daß in Region 2 eine Politik des hohen Schornsteins betrieben wird. D. h. in Region 2 wird versucht, durch vermehrten Emissionsexport nach Region 1 das Umweltproblem in der eigenen Region zu mindern.

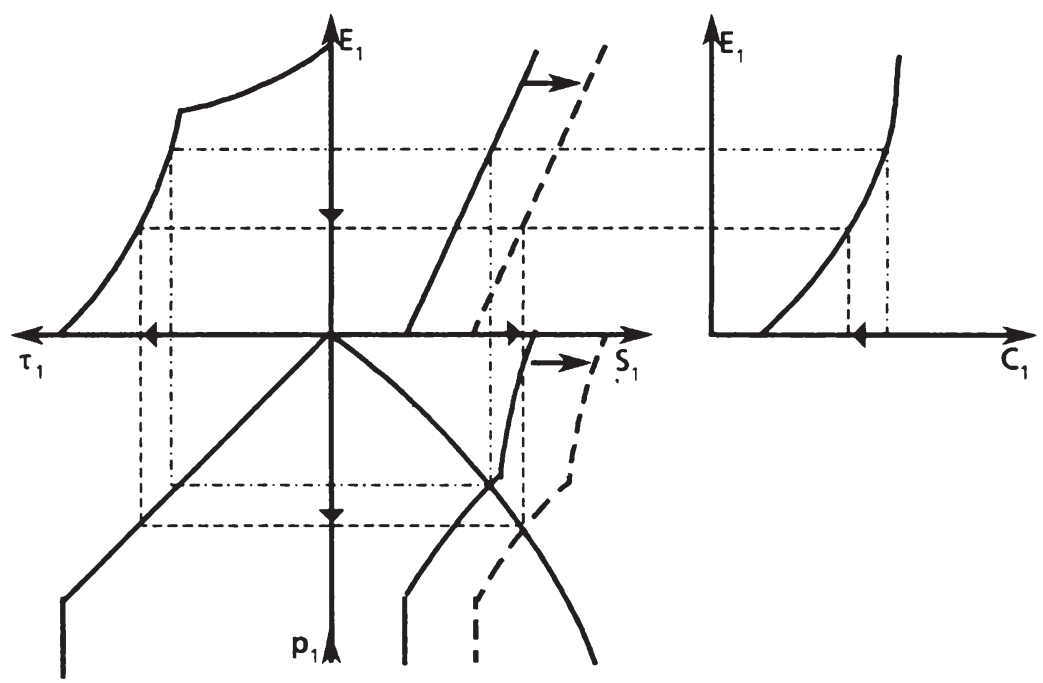

Schaubild 6.8: Änderungen in Region 1 bei erhöhtem Diffusionskoeffizienten

Wie aus Tabelle 6.1 ersichtlich, ergeben sich eindeutige Auswirkungen auf Region 1. Da die Emissionen in Region 2 steigen und dann ein größerer Anteil in das Ökosystem der Region 1 gelangt, muß Region 1 Wohlfahrtsverluste hinnehmen: Die Steady-State-Immissionen steigen und der Steady-State-Konsum sinkt, beides hat negative Wohlfahrtseffekte für Regi- 
on 1. In Schaubild 6.8 sind die Änderungen für Region 1 dargestellt. Die Pfeile geben die Änderungsrichtung an.

Für Region 2 erhalten wir eindeutige Effekte bezüglich der Emissionen $\left[d E_{2} / d \gamma_{2}>0\right]$, der Steuer $\left[d \tau_{2} / d \gamma_{2}<0\right]$ und des Konsums $\left[d C_{2} / d \gamma_{2}>0\right]$. Nicht eindeutig ist dagegen die Entwicklung der Immissionen und des Schattenpreises. Es gilt

$$
\frac{d S_{2}}{d \mathrm{Y}_{2}} \geq_{0}<\frac{d p_{2}}{d \mathrm{Y}_{2}} \leq_{0} \Leftrightarrow-\left(1-\mathrm{Y}_{2}\right) p_{2}+\left[u_{C}^{2} C_{E E}^{2}+u_{C C}^{2}\left(C_{E}^{2}\right)^{2} \mid E_{2<0} \geq_{0}\right.
$$

Dies ist äquivalent zu

$$
-\frac{d E_{2}}{d \tau_{2}} \frac{2}{E_{2}} \underset{1}{\gtrless}
$$

Ist die Steuerelastizität der Emissionen größer (kleiner) als Eins, d. h. die Emissionen sinken bei einer Steuererhöhung überproportional (unterproportional), dann nehmen die Immissionen bzw. nimmt der Zukunftsschaden bei einer exogenen Erhöhung des Diffusionsparameters zu (ab). Die Effekte einer Erhöhung des Diffusionskoeffizienten auf Region 2 sind in Schaubild 6.9 zusammengefaßt.

Betrachten wir zuerst den Fall der $d p_{2}{ }^{1} / d t=0$-Kurve. Diese Kurve verläuft relativ flach. Das bedeutet, daß die Umweltpolitik relativ schwach greift, schon in der Ausgangslage wird viel emittiert. Wird dann der Diffusionskoeffizient erhöht, steigen die Emissionen und die Steuer sinkt (eindeutige Effekte), jedoch sinken die Immissionen und der Zukunftsschaden $\left(p_{2}\right.$ steigt), und der Konsum steigt. In der Region 2 werden Wohlfahrtsgewinne realisiert. Im Fall der $d p_{2}{ }^{2} / d t=0$-Kurve sinkt zwar die Steuer ebenfalls und die Emissionen - und damit auch der Konsum - steigen, jedoch steigen auch die Immissionen und der Zukunftsschaden. Hier kann jetzt keine Aussage über die Wohlfahrtsänderung in Region 2 gemacht werden, da sich zwei entgegengesetzte Effekte gegenüberstehen. Durch die Immissionserhöhung ist ein negativer Wohlfahrtseffekt gegeben, während die Konsumsteigerung eine positive Wohlfahrtsänderung ergibt. Es kann also bei einer in der Ausgangslage strengen Umweltpolitik der Fall 


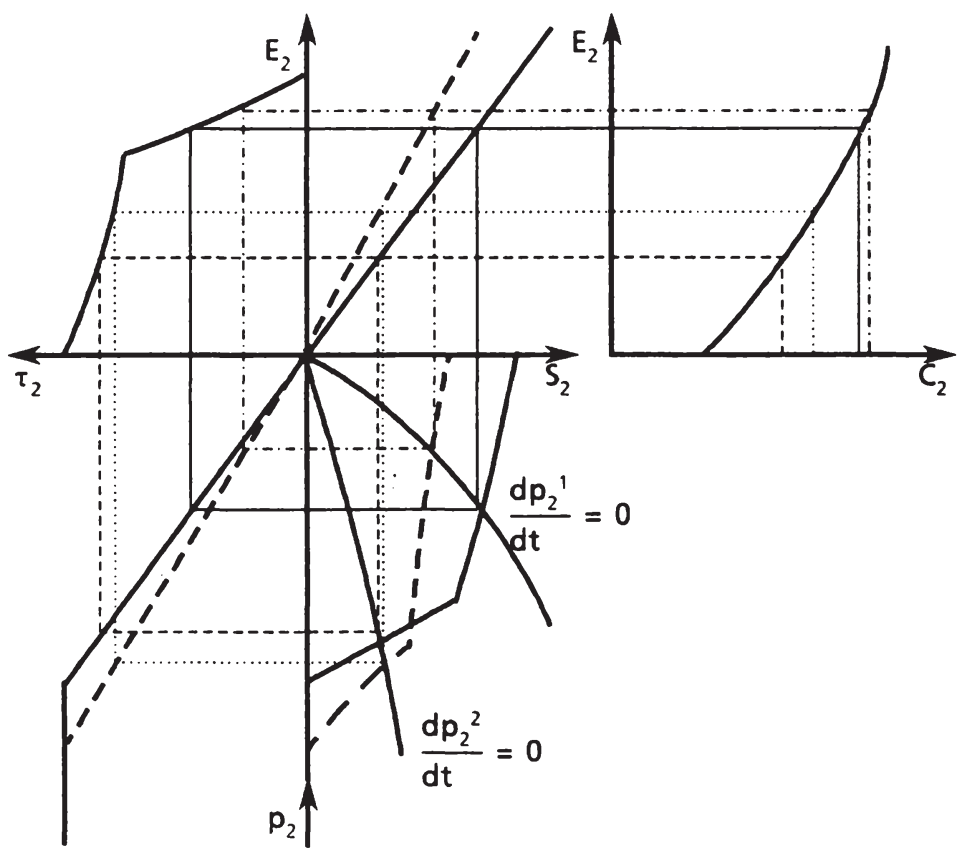

Schaubild 6.9: Die Effekte einer Diffusionskoeffizientenerhöhung auf Region 2

eintreten, daß durch eine Erhöhung des Diffusionskoeffizienten eine Wohlfahrtsminderung eintritt. Dies widerspricht sicherlich dem Anliegen einer Politik des hohen Schornsteins, mit Hilfe des Exports von Emissionen die Wohlfahrt zu verbessern. In Kapitel 4 wurde ein äquivalentes Ergebnis erzielt. Die Auswirkungen einer Politik des hohen Schornsteins auf die emissionsexportierende Region sind damit unabhängig davon, ob die Region als Bestandteil eines kleinen Landes über Handelsbeziehungen an einen Weltmarkt angebunden ist oder ob sie autark in ihrer Güterversorgung ist. 


\section{ZENTRALBEHÖRDE}

\subsection{DAS OPTIMIERUNGSPROBLEM UND DIE OPTIMALBEDINGUNGEN}

Die Zentralbehörde sieht sich folgendem Optimierungsproblem gegenüber

$$
\begin{aligned}
& \operatorname{Max} W=\int_{0}^{\infty}\left[\sum_{k=1}^{2} \beta_{k} u^{k}\left(C_{k}, S_{k}\right)\right] e^{-\delta t} d t \\
& \text { u.d.B. (3.1) } \quad f\left(R_{i}, E_{i}\right)-X_{i} \geq 0 \\
& \text { (3.2) } \quad a_{i} R_{i}-E_{i} \geq 0 \\
& \text { (6.4) } \quad C_{i}-X_{i}+q\left(R_{i}-R_{i}\right)=0 \\
& \text { (6.1.i) } \frac{d S_{1}}{d t}=E_{1}+\mathrm{Y}_{2} E_{2}-\mathrm{a}_{1} S_{1} \\
& \text { (6.1.ii) } \frac{d S_{2}}{d t}=\left(1-\mathrm{Y}_{2}\right) E_{2}-\mathrm{a}_{2} S_{2} \\
& C_{i}, X_{i}, R_{i}, E_{i} \geq 0 \quad \forall t \in[0, \infty), i=1,2 \\
& S_{i}(0)=S_{i}{ }^{0} \text { gegeben } \\
& \delta \text { gegeben und positiv }
\end{aligned}
$$

Hierauf wird die Methode der Kontrolltheorie angewandt und man erhält folgende Lagrange-Funktion in laufenden Werten:

$$
\begin{aligned}
& L=\sum_{k=1}^{2} B_{k}\left\{u^{k}\left(C_{k}, S_{k}\right)+\lambda^{k}{ }_{1}\left[f^{k}\left(R_{k}, E_{k}\right)-X_{k}\right]+\lambda^{k}{ }_{2}\left[a_{k} R_{k}-E_{k}\right]\right. \\
&+\lambda^{k}{ }_{3} C_{k}+\lambda^{k}{ }_{4} X_{k}+\lambda^{k}{ }_{5} R_{k}+\lambda^{k}{ }_{6} E_{k} \\
&\left.+\mu^{k}\left[C_{k}-X_{k}+q\left(R_{k}-R_{k}\right)\right]\right\} \\
&+\beta_{1} p_{1}\left[E_{1}+\gamma_{2} E_{2}-\alpha_{1} S_{1}\right]+B_{2} p_{2}\left[\left(1-\gamma_{2}\right) E_{2}-\alpha_{2} S_{2}\right]
\end{aligned}
$$

woraus sich folgende Bedingungen ergeben $(i=1,2)$ : 
(i) $\quad L_{C i}=B_{i}\left\{u_{C}^{i}+\lambda^{i}{ }_{3}+\mu^{i}\right\}=0$

$C_{i} \geq 0$

(ii) $\quad L_{X i}=B_{i}\left\{-\lambda_{1}^{i}+\lambda_{4}^{i}-\mu^{i}\right\}=0$

$X_{i} \geq 0$

(iii) $L_{R i}=B_{i}\left\{\lambda^{i}{ }_{1} f_{R}+\lambda^{i}{ }_{2} a_{i}+\lambda^{i}{ }_{5}+\mu^{i} \mathbf{q}\right\}=0$

$R_{i} \geq 0$

(iv.1) $L_{E 1}=B_{1}\left\{\lambda^{1}{ }_{1} f_{E}{ }_{E}-\lambda^{1}{ }_{2}+\lambda^{1}{ }_{6}+p_{1}\right\}=0$

$E_{1} \geq 0$

(iv .2) $L_{E 2}=B_{2}\left\{\lambda^{2}{ }_{1} \rho_{E}{ }_{E}-\lambda^{2}{ }_{2}+\lambda^{2}{ }_{6}+\left(1-\gamma_{2}\right) p_{2}\right\}+B_{1} \gamma_{2} p_{1}=0$

$E_{2} \geq 0$

(v.1) $L_{p 1}=\beta_{1} \frac{d S_{1}}{d t}=\beta_{1}\left\{E_{1}+\Upsilon_{2} E_{2}-a_{1} S_{1}\right\}$

(v.2) $L_{p 2}=\beta_{2} \frac{d S_{2}}{d t}=\beta_{2}\left\{\left(1-\mathrm{Y}_{2}\right) E_{2}-a_{2} S_{2}\right\}$

(6.25) (vi)

$$
L_{S i}=\beta_{i}\left\{-\frac{d p_{i}}{d t}+\delta p_{i}\right\}=\beta_{i}\left\{u_{S}^{i}-a_{i} p_{i}\right\}
$$

(vii)

$$
B_{i} \lambda^{\prime}{ }_{1}\left[\dot{f}\left(R_{i}, E_{i}\right)-X_{i}\right]=0
$$

$\lambda_{1}^{i} \geq 0$

(viii)

$$
B_{i} \lambda^{\prime}{ }_{2}\left[a_{i} R_{i}-E_{i}\right]=0
$$

$\lambda_{2}^{i} \geq 0$

(ix)

$$
B_{i} \lambda_{3}^{i} C_{i}=0
$$

$\lambda_{3}^{i} \geq 0$

(x)

$$
B_{i} \lambda^{i}{ }_{4} X_{i}=0
$$

$\lambda^{i} \geq 0$

(xi)

$$
B_{i} \lambda^{i} R_{5}=0
$$

$\lambda^{i}{ }_{5} \geq 0$

(xii)

$$
B_{i} \lambda{ }_{6}^{i} E_{i}=0
$$

$\lambda_{6}^{\prime} \geq 0$

(xiii)

$$
B_{i} \mu^{i}\left[C_{i}-X_{i}+\mathbf{q}\left(R_{i}-\mathbf{R}_{i}\right)\right]=0
$$

Auch hier wird davon ausgegangen, daß die Regionennutzen mit dem gleichen Gewicht in das Optimierungskalkül der Zentralbehörde eingehen. Es gilt $B_{1}=B_{2}=\frac{1}{2}$; damit können die Gewichte bei der folgenden Diskussion vernachlässigt werden.

Die Diskussion der Optimalbedingungen ist auch hier analog zu der in Kapitel 5, Abschnitt 2, so daß die Gleichungen (5.10), (5.12) und (5.14) weiterhin gelten.

Die Beziehung zwischen Steuer und Schattenpreis der Region 1 ist weiterhin durch Gleichung (6.9) gegeben. 
Die Beziehung zwischen Steuer und Schattenpreis der Region 2 ergibt sich analog zu Gleichung (4.27), d. h. es gilt

(6.26) $\quad \tau_{2}= \begin{cases}\tau_{2}^{\prime} & \text { für } p_{2} \leq p_{2}^{\prime}, p_{2}^{\prime} \equiv \frac{-\tau_{2}{ }^{\prime}}{1-\gamma_{2}}+\frac{\gamma_{2}}{1-\gamma_{2}} p_{1} \\ -\left(1-\gamma_{2}\right) p_{2}-\gamma_{2} p_{1} & \text { für } p_{2} \in\left(p_{2}^{\prime}, 0\right) \\ 0 & \text { für } p_{2}=0\end{cases}$

Hier werden also wiederum die Auswirkungen der Emissionen der Region 2 auf Region 1 berücksichtigt. In Analogie zu der in Kapitel 4, Abschnitt 3 vorgenommenen Analyse erhalten wir folgendes Differentialgleichungssystem [vgl. das Differentialgleichungssystem (4.28)]

(i)

$$
\frac{d S_{1}}{d t}=E^{1}\left(\tau_{1}\right)+\mathrm{v}_{2} E^{2}\left(\mathrm{\tau}_{2}\right)-\mathrm{a}_{1} S_{1}
$$

$$
\frac{d p_{1}}{d t}=\left(\delta+a_{1}\right) p_{1}-u_{S}^{1}
$$

$$
0=\tau_{1}+p_{1}
$$

$$
\frac{d S_{2}}{d t}=\left(1-\mathrm{Y}_{2}\right) E^{2}\left(\tau_{2}\right)-a_{2} S_{2}
$$

$$
\begin{aligned}
& \frac{d p_{2}}{d t}=\left(\delta+a_{2}\right) p_{2}-u_{S}^{2} \\
& 0=\tau_{2}+\left(1-\gamma_{2}\right) p_{2}+\gamma_{2} p_{1}
\end{aligned}
$$

Die formale Analyse von Kapitel 4 kann hier übernommen werden, da die Auswirkungen der Handelsbeziehungen der Ökonomie sich nur im Kurvenverlauf der $E^{i}\left(\tau_{i}\right)$-Funktionen zeigen. Für die Steady-State-Emissionsreaktionsfunktion der Region 2 in Abhängigkeit von den Emissionen der Region 1 erhält man $\left(E_{2} \neq E_{2}\right)$

$$
\frac{d E_{2}^{*}}{d E_{1}}=\frac{a_{2}\left(\delta+a_{2}\right) Y_{2} E_{\tau}^{2}}{E_{\tau}^{1} \Delta_{1}}>0
$$


Da die $E^{2}\left(\tau_{2}\right)$-Funktion in $E_{2}$ einen Knick hat [vgl. Fußnote 7, Kapitel 5], kann gezeigt werden, daß die $\varepsilon^{2}\left(E_{1}\right)$-Funktion in $E_{2}$ ebenfalls einen Knick hat, und zwar ist die Steigung bei der Annäherung von "unten" an $E_{2}\left(E_{2}\right.$ $\left.<E_{2}\right)$ größer als bei der Annäherung von "oben" $\left(E_{2}<E_{2}\right)^{4}$.

Diese Steady-State Reaktionsfunktion hat jedoch noch einen zweiten "Knick", der sich durch den Knick in der $E^{1}\left(\tau_{1}\right)$-Funktion überträgt. Bei der Annäherung von links an $E_{1}$ ist die Steigung der Reaktionsfunktion kleiner als bei der Annäherung von rechts.

Graphisch kann damit das Gesamt-Steady-State folgendermaßen dargestellt werden:

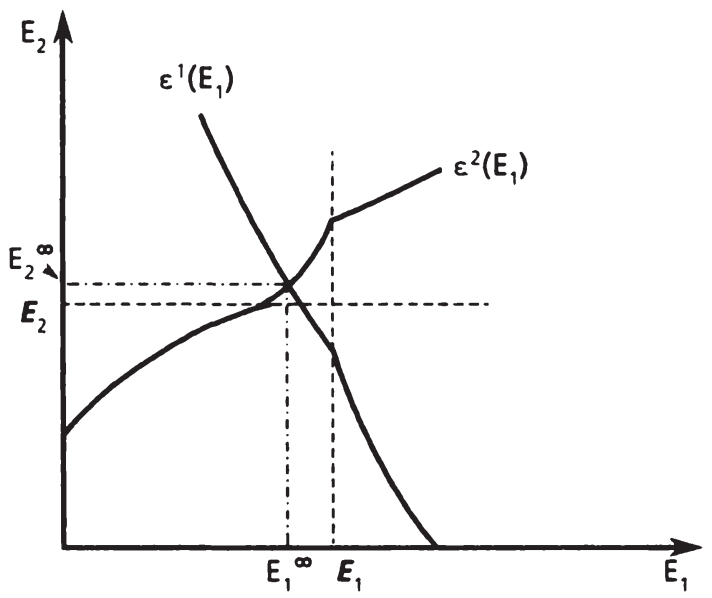

Schaubild 6.11: Die graphische Darstellung des Steady-States

4) Es gilt $E_{\tau}^{2+} \leq E_{\tau}^{2-} \Rightarrow \Delta_{2}^{+} \geq \Delta_{2}^{-} \Rightarrow \frac{d E_{2}^{*+}}{d E_{1}} \geq \frac{d E_{2}^{*}}{d E_{1}}$ 
Folgende Tabelle zeigt die Auswirkungen, die sich über die Größe der Parameter auf das Steady-State ergeben.

\begin{tabular}{|c|c|c|c|c|c|c|c|c|c|c|}
\hline & $d E_{1}$ & $d S_{1}$ & $d p_{1}$ & $d \tau_{1}$ & $d C_{1}$ & $d E_{2}$ & $d S_{2}$ & $d p_{2}$ & $d \tau_{2}$ & $d C_{2}$ \\
\hline$d \delta$ & $?$ & $\oplus$ & $?$ & $?$ & $?$ & $\oplus$ & $\oplus$ & $?$ & $\Theta$ & $\oplus$ \\
\hline$d \alpha_{1}$ & $\oplus$ & $?$ & $\oplus$ & $\Theta$ & $\oplus$ & $\oplus$ & $\oplus$ & $\Theta$ & $\Theta$ & $\oplus$ \\
\hline$d \mathbf{R}_{1}$ & $\Theta$ & $\Theta$ & $\oplus$ & $\Theta$ & $\oplus$ & $\oplus$ & $\oplus$ & $\Theta$ & $\Theta$ & $\oplus$ \\
\hline$d \alpha_{2}$ & $\Theta$ & $\oplus$ & $\Theta$ & $\oplus$ & $\Theta$ & $\oplus$ & $?$ & $\oplus$ & $\Theta$ & $\oplus$ \\
\hline$d \mathbf{R}_{2}$ & $\oplus$ & $\Theta$ & $\oplus$ & $\Theta$ & $\oplus$ & $\Theta$ & $\Theta$ & $\oplus$ & $\Theta$ & $\oplus$ \\
\hline$d \mathbf{q}$ & $?$ & $\Theta$ & $\oplus$ & $\Theta$ & $?$ & $?$ & $?$ & $?$ & $\Theta$ & $?$ \\
\hline $\begin{array}{c}d \gamma_{2} \\
\left(p_{2}>p_{1}\right)\end{array}$ & $?$ & $?$ & $?$ & $?$ & $?$ & $?$ & $\Theta$ & $\oplus$ & $?$ & $?$ \\
\hline
\end{tabular}

Tabelle 6.2: Auswirkungen von Parameteränderungen bei einer Zentralregierung

\subsubsection{Die Auswirkungen von $\delta, a_{i}$ und $\gamma_{2}$}

Ein Vergleich der Vorzeichen der Tabelle 6.2 und der Tabelle 4.2 zeigt, daß die Auswirkungen der sozialen Zeitpräferenzrate, der Assimilationsfähigkeit der Ökosysteme und des Diffusionskoeffizienten äquivalent sind zu denen ohne Handelsbeziehungen [vgl. auch die analytischen Ableitungen in den Anhängen 4.II und 6.II].

Die Auswirkungen der Höhe dieser Parameter auf das Steady-State sind also weitgehend unabhängig von den Handelsbeziehungen. Die Größe dieser Parameter hat auf das Steady-State - zumindest der Richtung nach die gleichen Auswirkungen, ob die Faktoren und Güter mobil oder immobil sind. Hier ist die Diffusionsbeziehung zwischen den Regionen entscheidend, welche Änderungsrichtung die Steady-State-Variablen bei Variation dieser Parameter haben. Für die Diskussion im einzelnen kann auf die Abschnitte 3.3.1, 3.3.2 und 3.3.4 des Kapitels 4 verwiesen werden, da sich hier die vollkommen äquivalenten Bedingungen ergeben. 


\subsubsection{Die Auswirkungen der Höhe der Ressourcenausstattungen}

Auch hier ergibt sich, genau wie im Fall autonomer Regionalbehörden, eine Umkehr der Vorzeichen gegenüber dem Fall autarker Regionen. Betrachten wir zuerst die Ressourcenausstattung der Region 1:

Je größer der Faktorbestand der Region 1 ist, desto weniger wird in Region 1 produziert und damit emittiert. Die Emissionssteuer wird kleiner, jedoch der Konsum steigt. Obwohl der Emissionsimport aus Region 2 steigt, sinken die Immissionen und der Schaden $\left(p_{1}\right.$ steigt).

Auf Region 2 hat die Höhe der Faktorausstattung der Region 1 ebenfalls Auswirkungen. Die Emissionen, die Immissionen und der Konsum steigen, während der Schaden und die Steuer sinken. Dieses Ergebnis ist überraschend, da wir für die Emissionsreaktionsfunktion der Region 2 einen positiv steigenden Verlauf abgeleitet haben, $d$. h. daß je niedriger die Emissionen in Region 1 sind, auch die Emissionen in Region 2 umso niedriger sein müssen. Hier ist jetzt der umgekehrte Fall gegeben. Durch den höheren Faktorbestand in Region 1 sinken zwar die Emissionen in Region 1 , jedoch sinkt auch die Bewertung des Schadens in Region $1\left[d p_{1} / d \mathbf{R}_{1}\right.$ $>0]$. Die Bewertung des Schadens der Region 2 bei konstanten Emissionen sinkt damit auch in Region 2. Durch die Beziehung $E_{2}=E^{2}\left(\tau_{2}\right)$ steigen jedoch dann die Emissionen in Region 2. Es erfolgt also eine Verschiebung der Steuergeraden von Region 2. In Schaubild 6.11 sind die Effekte zusammengefaßt. Die Pfeile geben die Änderungsrichtung an.

Betrachten wir nun die Effekte der Höhe der Faktorausstattung der Region 2:

Analog zum Fall ohne Diffusion [vgl. Kapitel 5], sinken die Emissionen, die Immissionen, die Steuer und die Bewertung des Schadens $\left[p_{2}\right.$ wird größer] in Region 2, je größer die Faktorausstattung der Region 2 ist. Für Region 1 ergibt sich, daß die Emissionen und der Konsum steigen, während die Immissionen, die Steuer und die Bewertung des Schadens sinken [ $p_{1}$ wird größer]. Diese Effekte auf Region 1 ergeben sich durch die verminderten Emissionen in Region 2, durch die Region 1 in der Lage ist, vermehrt zu emittieren und gleichzeitig die Immissionen bei höherem Konsum zu senken.

Die Höhe des Faktorbestandes der Region 2 bei einer Zentralregierung hat 


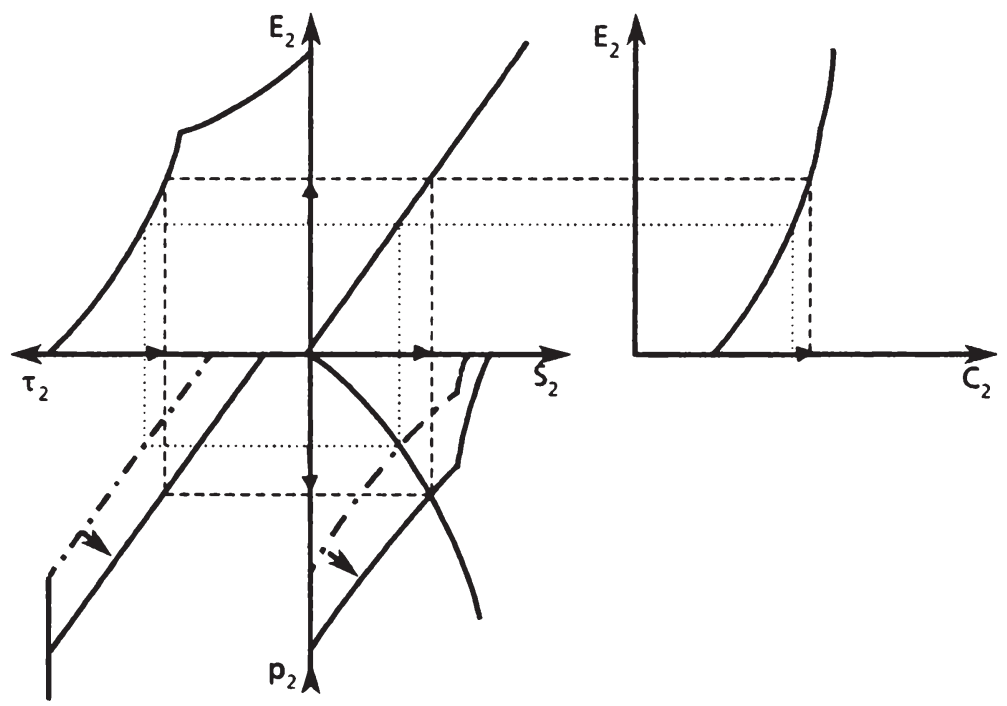

Schaubild 6.11: Die Änderungen in Region 2 bei erhöhtem Faktorbestand in Region 1

auf die Steady-State Variablen der Richtung nach die äquivalenten Auswirkungen wie bei autonomen Regionen [vgl. Abschnitt 2.3.4 dieses Kapitels] und genau die entgegengesetzten Auswirkungen wie für immobile Güter und Faktoren, unabhängig von dem institutionellen Arrangement [vgl. die Ausführungen in Kapitel 4].

\subsubsection{Die Auswirkungen der Höhe des Relativpreises auf das Steady- State}

Je höher der Relativpreis ist, desto niedriger sind die Immissionen, die Steuer und der Schaden in Region 1 und auch die Steuer in Region 2 ist niedriger. Für die Änderungsrichtungen der anderen Variablen $\left(E_{1}, C_{1}\right.$, $E_{2}, S_{2}, p_{2}, C_{2}$ ) kann kein eindeutiges Vorzeichen abgeleitet werden.

Betrachten wir zuerst Region 2:

Die Änderung der Emissionen, der Immissionen und des Schadens in Region 2 bei einem steigenden Relativpreis kann nicht bestimmt werden. Sie können sowohl steigen, gleich bleiben oder fallen. 
Für die Änderungsrichtung des Konsumฐin Region 2 bei steigendem Relativpreis ist neben der Änderungsrichtung der Emissionen auch die Faktorausstattung der Region 2 relevant. Steigen (sinken) die Emissionen in Region 2 und ist Region 2 Faktorexporteur (-importeur), dann steigt (sinkt) der Konsum in Region 2 bei steigendem Relativpreis.

Die Änderungsrichtungen in Abhängigkeit der Änderungsrichtung der Emissionen und in Abhängigkeit der Handelsposition können folgendermaßen zusammengefaßt werden ${ }^{5)}$ :

\begin{tabular}{|c|c|c|c|}
\hline$\frac{d C_{i}}{d q}$ & $\frac{d E_{i}}{d q}>0$ & $\frac{d E_{i}}{d q}=0$ & $\frac{d E_{i}}{d q}<0$ \\
\hline$M_{i}>0$ & $?$ & $<0$ & $<0$ \\
\hline$M_{i}=0$ & $>0$ & $=0$ & $<0$ \\
\hline$M_{1}<0$ & $>0$ & $>0$ & $?$ \\
\hline
\end{tabular}

Tabelle 6.3: Die mögliche Änderungsrichtungen des Konsums

Sind die Emissionen trotz Relativpreissteigerung konstant, dann ist die Änderungsrichtung des Konsums nur von der Handelsposition der Region abhängig. Ist Region 2 Ressourcenimporteur $-M_{2}>0$ - (-exporteur $M_{2}<0-$ ), dann sinkt (steigt) der Konsum in dieser Region.

Steigen dagegen die Emissionen, dann steigt der Konsum, wenn die Region Faktoren exportiert. Ist die Region andererseits Faktorimporteur, dann kann es trotz Steigerung der Emissionen vorkommen, daß der Konsum sinkt. Hat also Region 2 wenig Faktoren als Grundausstattung und sie muß einen hohen Betrag an Faktoren importieren, dann müssen in dieser Region Wohlfahrtsverluste hingenommen werden. Sinken die Emissionen, ist der diametral entgegengesetzte Fall zum Fall steigender Emissionen gegeben: Trotz Senkung der Emissionen und der Produktion ${ }^{6}$ kann

5) Da für Region 1 bezüglich des Konsums eine analoge Argumentation gilt, sind die Subskripte in der Tabelle mit $i=1,2$ ausgewiesen.

6) $d R_{2} / d q<0$ für $d E_{2} / d q<0$, so daß sowohl die eingesetzten Produktionsfaktoren als auch die Emissionen sinken und damit auch die Produktion des Gutes. 
bei genügend hoher Faktorausstattung der Region der Konsum gesteigert werden.

Die Änderung der Emissionen in Region 1 sind abhängig davon, ob die Emissionen in Region 2 steigen oder fallen. Da die Steady-State-Immissionen in Region 1 sinken, müssen die Emissionen in Region 1 ganz sicher dann sinken, wenn die Emissionen in Region 2 nicht fallen und zwar müssen sie stärker sinken als der Anteil der aus Region 2 importierten Emissionen steigt, da gilt

$$
\frac{d E_{1}}{d \mathbf{q}}<-\mathrm{Y}_{2} \frac{d E_{2}}{d \mathbf{q}}
$$

Andererseits können die Emissionen in Region 1 nicht so stark steigen, wie der Anteil der aus Region 2 importierten Emissionen sinkt.

Für den Konsum der Region 1 erhält man eine analoge Argumentation wie für Region 2: Der Konsum kann dann steigen, wenn die Emissionen in Region 1 steigen können und die Region Faktorexporteur ist oder wenn die Emissionen sinken und der Faktorbestand genügend hoch ist, so daß weniger produziert wird und die Faktoren gegen Konsumgüter eingetauscht werden. 


\section{AUTONOME REGIONALREGIERUNGEN MIT ZENTRALER STANDARDSETZUNG}

Auch hier wird, in Analogie zu Kapitel 4, untersucht, welche Änderungen sich ergeben, wenn die Regionen weiterhin autonom sind, jedoch eine Zentralbehörde eingreift, um die sozialen Kosten dem Verursacher zuzuordnen. Die Zentralbehörde gibt wieder für Region 2 einen Emissionsstandard vor, den die Emissionen von Region 2 nicht überschreiten dürfen.

Das Optimierungsproblem für Region 1 ändert sich gegenüber demjenigen im autonomen Fall nicht, so daß hier wieder nur die Änderungen für Region $2 \mathrm{zu}$ betrachten sind.

\subsection{DAS OPTIMIERUNGSPROBLEM FÜR REGION 2 UND DIE OPTIMALBEDINGUNGEN}

Es gilt hier Gleichung (4.36)

$$
E_{2}(t) \leq \mathbf{E}_{2} \quad \forall t \in[0, \infty)
$$

Damit hat die Regionalbehörde von Region 2 folgendes Optimierungsproblem zu lösen:

$$
\begin{aligned}
& \operatorname{Max} W_{2}=\int_{0}^{\infty} u^{2}\left(C_{2}, S_{2}\right) e^{-\delta t} d t \\
& \text { u.d.B. (3.1) } \quad f^{2}\left(R_{2}, E_{2}\right)-X_{2} \geq 0 \\
& \begin{aligned}
\text { (3.2) } & a_{2} R_{2}-E_{2} \geq 0 \\
(6.29) & E_{2}-E_{2} \geq 0 \\
(6.4) & C_{2}-X_{2}+q\left(R_{2}-R_{2}\right)=0 \\
(6.1 . i i) & \frac{d S_{2}}{d t}=\left(1-\gamma_{2}\right) E_{2}-a_{2} S_{2} \\
& X_{2}, C_{2}, R_{2}, E_{2} \geq 0 \forall t \in[0, \infty) \\
& S_{2}(0)=S_{2}{ }^{0} \text { gegeben } \\
& \delta \text { gegeben und positiv }
\end{aligned}
\end{aligned}
$$


Daraus folgt für die Lagrange-Funktion in laufenden Werten:

$$
\begin{aligned}
L^{2}=u^{2}\left(C_{2}, S_{2}\right) & +p_{2}\left[\left(1-\gamma_{2}\right) E_{2}-\alpha_{2} S_{2}\right] \\
& +\lambda^{2}\left[f^{2}\left(R_{2}, E_{2}\right)-X_{2}\right]+\lambda^{2}{ }_{2}\left[a_{2} R_{2}-E_{2}\right]+\lambda^{2}{ }_{3}\left[E_{2}-E_{2}\right] \\
& +\lambda^{2}{ }_{4} C_{2}+\lambda^{2}{ }_{5} R_{2}+\lambda^{2}{ }_{6} E_{2}+\lambda^{2}{ }_{7} X_{2} \\
& +\mu^{2}\left[C_{2}-X_{2}+\mathbf{q}\left(R_{2}-\mathbf{R}_{2}\right)\right]
\end{aligned}
$$

Woraus sich folgende Optimalbedingungen ergeben:

$$
L_{C}^{2}=u_{C}^{2}+\lambda^{2}{ }_{4}+\mu^{2}=0
$$$$
c_{2} \geq 0
$$

$$
L^{2}{ }_{X}=-\lambda^{2}{ }_{1}+\lambda^{2}{ }_{7}-\mu^{2}=0
$$$$
x_{2} \geq 0
$$

$$
L^{2}{ }_{R}=\lambda^{2}{ }_{1} f_{R}{ }_{R}+\lambda^{2}{ }_{2} a_{2}+\lambda^{2}{ }_{5}+\mathrm{q} \mu^{2}=0
$$$$
R_{2} \geq 0
$$

$$
L_{E}^{2}=\left(1-\gamma_{2}\right) p_{2}+\lambda^{2}{ }_{1} P_{E}-\lambda^{2}{ }_{2}-\lambda^{2}{ }_{3}+\lambda^{2}{ }_{6}=0
$$$$
E_{2} \geq 0
$$

(v)

$$
L_{p}^{2}=\frac{d S_{2}}{d t}=\left(1-\gamma_{2}\right) E_{2}-a_{2} S_{2}
$$

$$
L_{S}^{2}=-\frac{d p_{2}}{d t}+\delta p_{2}=u_{S}^{2}-a_{2} p_{2}
$$

(6.32)

$$
\text { (vii) }
$$

$$
\lambda^{2}{ }_{1}\left[P^{P}\left(R_{2}, E_{2}\right)-X_{2}\right]=0
$$

$$
\lambda_{2}^{2}\left[a_{2} R_{2}-E_{2}\right]=0
$$$$
\lambda^{2}{ }_{2} \geq 0
$$

$$
\lambda^{2}{ }_{3}\left[E_{2}-E_{2}\right]=0
$$$$
\lambda^{2}{ }_{3} \geq 0
$$

$$
\lambda^{2}{ }_{4} C_{2}=0
$$$$
\lambda^{2}{ }_{4} \geq 0
$$

$$
\lambda^{2}{ }_{5} R_{2}=0
$$$$
\lambda^{2}{ }_{5} \geq 0
$$

$$
\lambda^{2}{ }_{6} E_{2}=0
$$$$
\lambda_{6}^{2} \geq 0
$$

$$
\lambda^{2}{ }_{7} X_{2}=0
$$$$
\lambda^{2}{ }_{7} \geq 0
$$

(xiv)

$$
\mu^{2}\left[C_{2}-X_{2}+q\left(R_{2}-R_{2}\right)\right]=0
$$

Annahmegemäß gilt wieder $C_{2}>0 \forall t \in[0, \infty)$ auf dem Optimalpfad. Damit ist $\lambda^{2}{ }_{4}=0$ und es gilt

$$
u_{C}^{2}=-\mu^{2}>0 .
$$

Damit gilt 


$$
C_{2}=X_{2}-\mathbf{q}\left(R_{2}-R_{2}\right)>0
$$

Gehen wir davon aus, daß $X_{2}>0$ ist, dann ist auch $R_{2}>0$ und damit $\lambda^{2}{ }_{5}$ $=\lambda^{2}{ }_{7}=0$. Dann gilt wiederum

$$
-\mu^{2}=\lambda_{1}^{2}>0
$$

und damit folgt

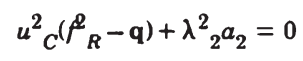

Wir erhalten

$$
\lambda^{2}{ }_{2}=-\frac{u_{C}^{2}}{a_{2}}\left(\tilde{f}_{R}-q\right) \quad \begin{cases}=0 & \text { für } E_{i}<a_{2} R_{2} \\ \geq 0 & \text { für } E_{i}=a_{2} R_{2}\end{cases}
$$

Aus dieser Beziehung kann in Analogie zu Gleichung (5.7) wieder eine Beziehung zwischen dem Faktoreinsatz und den Emissionen abgeleitet werden:

$$
R_{2}=\left\{\begin{array}{lll}
R^{2}\left(E_{2}\right), & R_{E}^{2}=-\frac{f_{R E}^{2}}{f_{R R}}>0 & \text { für } E_{2}<E_{2} \\
\frac{1}{a_{2}} E_{2} & & \text { für } E_{2} \geq E_{2}
\end{array}\right.
$$

wobei $E_{2}$ derjenige Emissionsbetrag ist, für den $E_{2}=a_{2} R_{2}$ und $f_{R}=q$ gilt.

Daraus ergibt sich eine Beziehung zwischen Konsum und Emissionen [vgl. Gleichung (5.12)]

$$
C_{2}=C^{2}\left(E_{2}\right)
$$

mit den gleichen Eigenschaften wie Beziehung (5.12). 
Aus den Optimalbedingungen (6.32.iv) ergibt sich

$$
\left(1-\gamma_{2}\right) p_{2}+u_{C}^{2} C_{E}^{2}-\lambda_{3}^{2}+\lambda_{6}^{2}=0
$$

Im folgenden wird davon ausgegangen, daß der Emissionsstandard für Region 2 bindend ist. Dann gilt

$$
E_{2}=E_{2}>0
$$

und damit $\lambda_{6}^{2}=0$.

Da damit die Emissionen im Zeitablauf konstant sind, ist wegen der Konstanz des Relativpreises, auch der Faktoreinsatz konstant und deshalb wird auch der Konsum auf

$$
C_{2}=\mathrm{C}_{2}
$$

festgelegt.

Aus der Beziehung zwischen Steuer und Emissionen ergibt sich damit

$$
\tau_{2}=u_{C}^{2} C^{2}{ }_{E}=\tau_{2}
$$

Die Beziehung zwischen der Steuer und dem Schattenpreis ist dann

$$
\tau_{2}=-\left(1-\gamma_{2}\right) p_{2}+\lambda^{2}{ }_{3}
$$

Die Steuer ist also wieder aus zwei Teilen zusammengesetzt: Einmal drückt sie den Schaden aus, der sich durch die Emissionstätigkeit ergibt und zum anderen wird sie erhöht durch die Kosten der Einhaltung des Emissionsstandards [vgl. die Diskussion zu Gleichung (4.4.1)].

Die Auswirkungen der Emissionsstandardsetzung auf Region 2 können in zwei Fälle unterschieden werden:

(1.) Das unbeschränkte Optimum $E^{a}{ }_{2}$ für Region 2 liegt im Bereich $E^{a}{ }_{2}<$ $E_{2}$. Für einen bindenden Emissionsstandard gilt

$$
E_{2}<E^{a}{ }_{2}<E_{2}
$$


Aus Gleichung (6.36) erhält man $\lambda^{2}{ }_{2}=0$ und deshalb

$$
f_{R}=\mathrm{q} ;
$$

das Wertgrenzprodukt des Faktors ist dann weiterhin gleich seinem Preis. Jedoch ist der Faktoreinsatz kleiner und damit die Produktion als im unbeschränkten Optimum; damit ist auch der Konsum kleiner [vgl. Schaubild 6.12].

(2.) Das unbeschränkte Optimum liegt in dem Bereich $E^{a}{ }_{2}>E_{2}$. Für den bindenden Emissionsstandard soll gelten ${ }^{7)}$

$$
E^{a}{ }_{2}>E_{2}>E_{2}
$$

Hier gilt nicht mehr $f_{R}=q$, sondern

$$
f_{R}<\mathbf{q}
$$

und

$$
\mathrm{E}_{2}=a_{2} R_{2}
$$

Auch hier sinkt der Faktoreinsatz und die Produktion gegenüber dem unbeschränkten Optimum in Region 2 und auch der Konsum sinkt [vgl. Schaubild 6.13].

Durch das Eingreifen der Zentralregierung über eine Emissionsstandardsetzung wird die Handelsposition der Region 2 maßgeblich mitbestimmt. Ist die Begrenzung der Emissionen genügend hoch, kann es sein, daß Region 2 vom Faktorimporteur zum Faktorexporteur wird. Im Gegensatz zum autarken Fall des Kapitels 4, kann Region 2 jedoch den Produktionsrückgang entweder durch vermehrten Faktorexport oder verminderten Faktorimport teilweise ausgleichen, so daß die Konsummenge weniger stark sinkt wie im autarken Fall.

7) Ist $E_{2}^{a}>E_{2}>E_{2}$, dann gilt für das unbeschränkte Optimum $E_{2}=a_{2} R_{2}$, während für das Optimum bei Standardsetzung $f_{R}=q$ gilt. Die Produktion und der Konsum sinken jedoch in jedem Fall. 


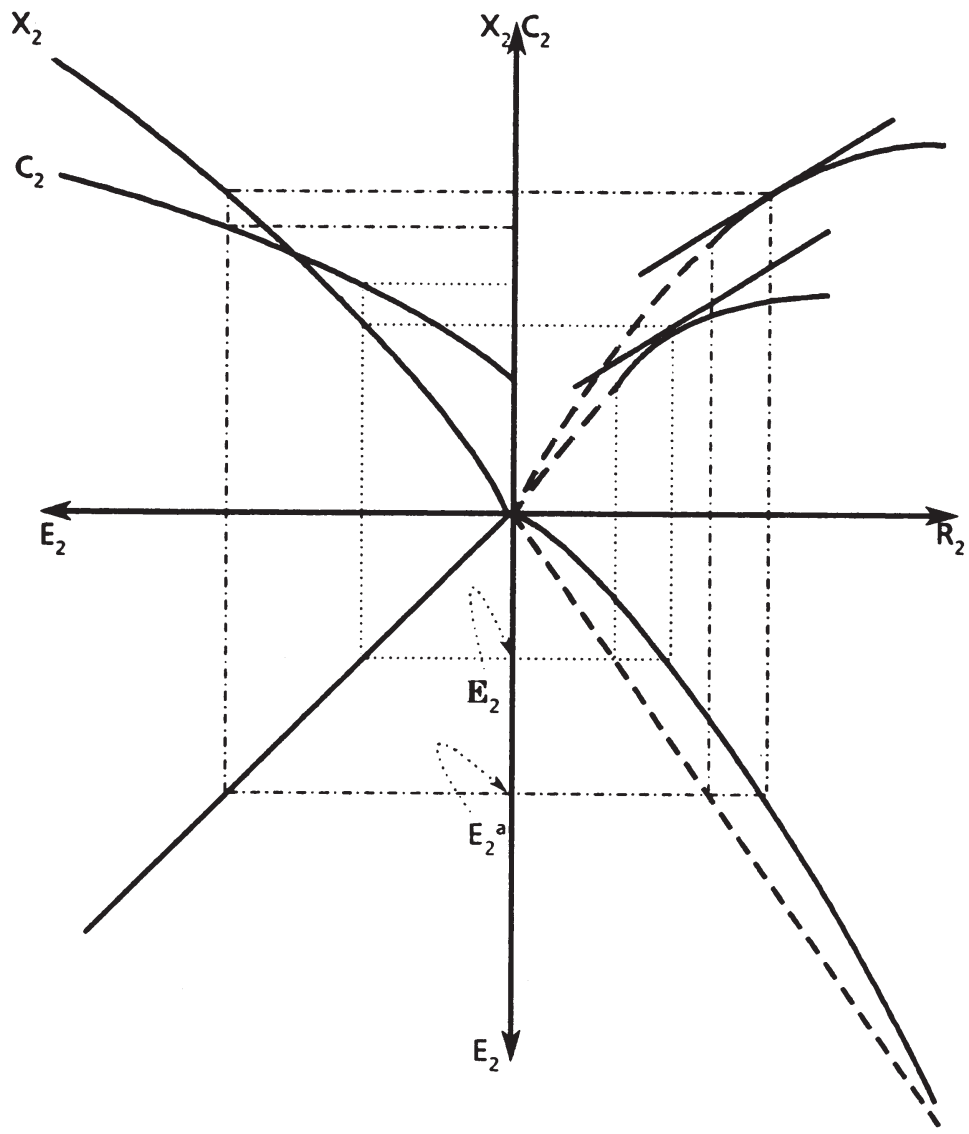

Schaubild 6.12: Die Auswirkungen der Standardsetzung auf Region 2

Zu der Analyse der Anpassungen an das Steady-State vergleiche die Analyse in Kapitel 4, Abschnitt 4.1, die hier analog erfolgt, so daß auf eine Diskussion verzichtet werden kann. 


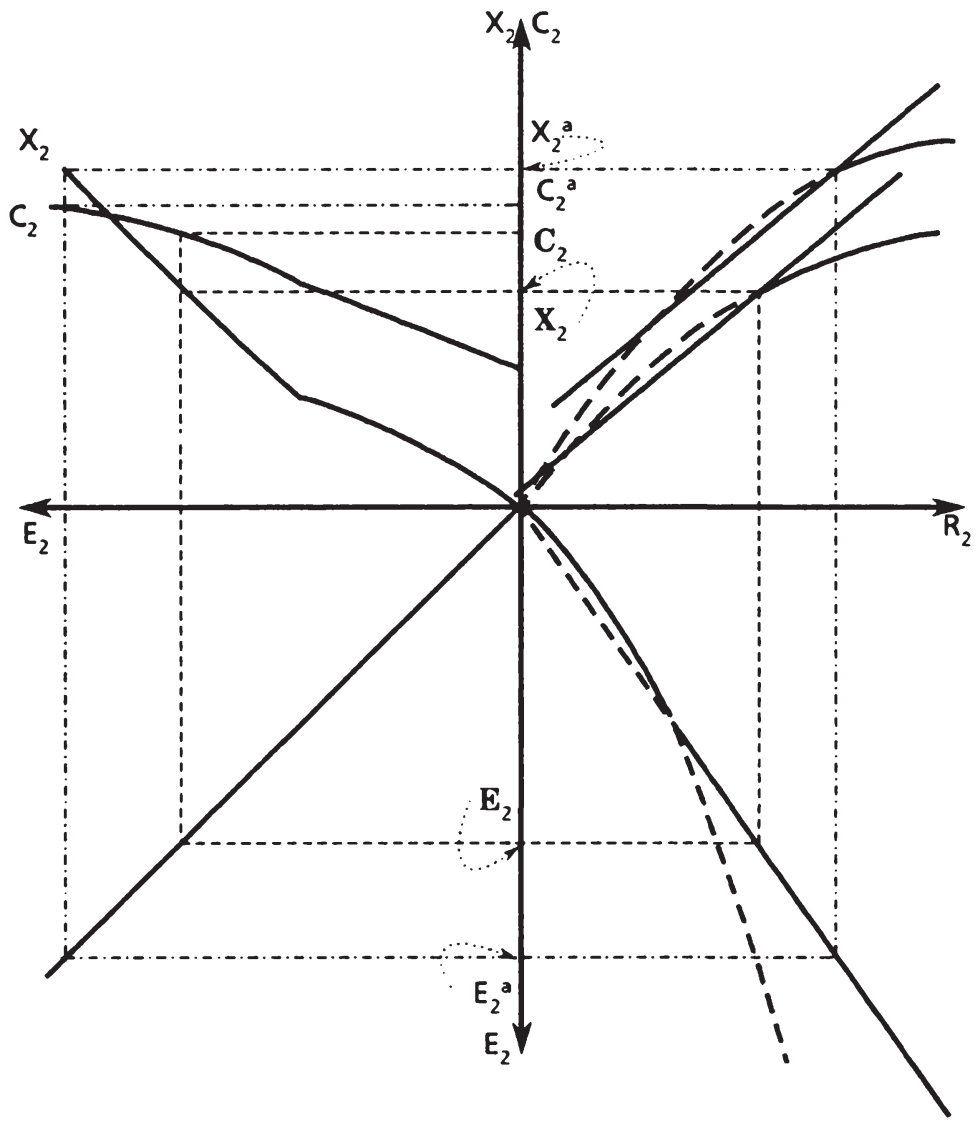

Schaubild 6.13: Die Auswirkungen der Standardsetzung auf Region 2 bei Ausnutzung der Kuppelproduktionsgrenze 
4.2 DiE AUSWIRKUNGeN DER GRÖßE DER PARAMETER AUF DAS STEADY-STATE

Die Auswirkungen von Parameteränderungen auf die Steady-State-Variablen sind in folgender Tabelle zusammengefaßt.

\begin{tabular}{|c|c|c|c|c|c|c|c|c|c|c|}
\hline & $d E_{1}$ & $d S_{1}$ & $d p_{1}$ & $d \tau_{1}$ & $d C_{1}$ & $d S_{2}$ & $d p_{2}$ & $d \tau_{2}$ & $d \lambda^{2}{ }_{3}$ & $d C_{2}$ \\
\hline$d \delta$ & $\oplus$ & $\oplus$ & $\oplus$ & $\Theta$ & $\oplus$ & 0 & $\oplus$ & 0 & $\oplus$ & 0 \\
\hline$d \alpha_{2}$ & 0 & 0 & 0 & 0 & 0 & $\Theta$ & $\oplus$ & 0 & $\Theta$ & 0 \\
\hline$d \mathbf{R}_{2}$ & 0 & 0 & 0 & 0 & 0 & 0 & 0 & $\Theta$ & $\Theta$ & $\oplus$ \\
\hline$d \mathbf{q}$ & $\Theta$ & $\Theta$ & $\oplus$ & $\Theta$ & $?$ & 0 & 0 & $\Theta$ & $\Theta$ & $?$ \\
\hline$d \gamma_{2}$ & $\Theta$ & $\oplus$ & $\Theta$ & $\oplus$ & $\Theta$ & $\Theta$ & $\oplus$ & 0 & $\oplus$ & 0 \\
\hline$d \mathbf{E}_{2}$ & $\Theta$ & $\oplus$ & $\Theta$ & $\oplus$ & $\Theta$ & $\oplus$ & $\Theta$ & $\Theta$ & $\Theta$ & $\oplus$ \\
\hline
\end{tabular}

Tabelle 6.4: Die Änderungen des Steady-States in Abhängigkeit der Größe der Parameter der Region 2

\subsection{1 $\quad$ Region 2}

Ein Vergleich mit den Vorzeichen der Tabelle 4.3 ergibt, daß die Handelsbeziehungen für die Änderungen der Steady-State-Werte bezüglich des Diffusionskoeffizienten, des Emissionsstandards, der Assimilationsfähigkeit und der Diskontrate von zweitrangiger Bedeutung sind, da sich die gleichen Vorzeichen wie im autarken Fall ergeben. Bei der Abhängigkeit von der Ressourcenausstattung der Region 2 ergeben sich jedoch Änderungen. Die Immissionen und die daraus resultierenden Schäden sind weiterhin unabhängig von der Faktorausstattung der Region bei bindendem Emissionsstandard, jedoch ist die Emissionssteuer umso niedriger, je mehr Faktoren in der Region als Grundausstattung vorhanden sind, da die Kosten des Konsumverzichts $\lambda^{2}{ }_{3}$ sinken und der Konsum steigt. In dem in Abschnitt 2 dieses Kapitels analysierten Fall autonomer Regionalbehörden hatten wir das Ergebnis, daß je mehr Faktoren die Region 2 besitzt, sie umso weniger in den eigenen Grenzen produziert, sondern die Produktion immer mehr nach "draußen" verlagert. Diese Verlagerung der 
Produktion, die bei Standardsetzung sogar vermehrt auftritt, da weniger Faktoren im Produktionsprozeß der Region 2 eingesetzt werden dürfen als "regionenegoistisch" optimal ist, vermindert die Kosten des Konsumverzichts und damit die Steuer in Region 2.

Diese Wirkungen der Größe der Faktorausstattung auf die Steuer und die Kosten des Konsumverzichts sind den in Kapitel 4, Absatz 4.2 abgeleiteten Auswirkungen genau entgegengesetzt, da bei autarken Regionen die emissionsexportierende Region bei einem bindenden Emissionsstandard keine Möglichkeiten hat, die Produktion zu verlagern, sondern die Faktoren alle in den Produktionsprozeß einsetzt, um bei konstanten Emissionen mehr zu produzieren.

Die Auswirkungen der Höhe des Relativpreises auf die Steady-State Variablen sind in der vierten Zeile der Tabelle 6.4 aufgelistet. Da der Emissionsstandard weiterhin bindend sein soll, verändern sich die Immissionen und der Schattenpreis, d. h. der bewertete Schaden aus der Emissionstätigkeit, nicht. Je höher der Relativpreis ist, desto niedriger ist die Steuer in Region 2 und desto niedriger sind die Kosten des Konsumverzichts. Die Änderung des Konsums ist abhängig davon, ob die Region Faktorimporteur oder Faktorexporteur ist. Ist die Region Faktorimporteur, dann müssen wegen der Verteuerung des Faktors relativ zum Konsumgut mehr Konsumgüter verkauft werden, um die gleiche Menge an Faktoren einsetzen zu können. Da die Produktion für einen bindenden Emissionsstandard konstant ist, müssen mehr Güter verkauft werden und damit sinkt der Konsum in der Region. Dieses Ergebnis ist äquivalent zu dem der autonomen Regionalbehörden [vgl. Abschnitt 2.3.4 dieses Kapitels].

Ist die Region dagegen Ressourcenexporteur, steigt der Konsum mit steigendem Relativpreis. Für die gleiche Menge an Faktoren, die die Region verkauft, erhält sie eine größere Menge an Konsumgütern, so daß in der Region mehr konsumiert wird. Unabhängig von der Handelsposition der Region sinken die Kosten des Konsumverzichts für steigenden Relativpreis, da, wenn einerseits der Konsum steigt (die Region ist Faktorexporteur), man näher an die "regionenegoistisch" optimale Konsummenge herankommt, während andererseits, wenn die Region Faktorimporteur ist, der Konsum zwar sinkt, jedoch die Senkung weniger stark ist wie bei auto- 
nomen Regionalregierungen [vgl. die Gleichungen in Anhang 6.III], so daß die Kosten des Konsumverzichts sinken.

\subsection{2 $\quad \underline{\text { Region } 1}$}

Die soziale Zeitpräferenzrate $\delta$ hat die äquivalenten Auswirkungen auf die Steady-State Variablen der Region 1 wie im Fall ohne Diffusion [vgl. Kapitel 5, Abschnitt 2.3.3]: die Emissionen, die Immissionen und der Konsum sind umso höher und die Steuer und die Bewertung des Schadens sind umso niedriger, je höher die soziale Diskontrate ist. Die Änderung der Assimilationskapazität des Ökosystems der Region 2 und der Faktorausstattung der Region 2 haben bei einem bindenden Emissionsstandard keine Auswirkungen auf die Steady-State Variablen der Region 1. Diese Unabhängigkeit der emissionsimportierenden Region von der emissionsexportierenden Region hat sich auch bei den Untersuchungen von autarken Regionen ergeben, so daß hier die Handelsbeziehungen von zweitrangiger Bedeutung sind. Auch die Auswirkungen einer Erhöhung des Relativpreises sind vollkommen analog zu denen ohne Diffusionsbeziehungen zwischen den Regionen: die Emissionen, die Immissionen, die Steuer und die Bewertung des Schadens sinken. Die Änderung des Konsums ist wieder von Bedingung (5.20) abhängig, so daß die in Kapitel 5, Abschnitt 4.3 gemachten Aussagen gültig bleiben.

Die Auswirkungen einer Erhöhung des Diffusionskoeffizienten und des Emissionsstándards ergeben sich analog zu den in Kapitel 4, Abschnitt 4.2.2 abgeleiteten Auswirkungen: die Emissionen und der Konsum sinken, während die Immissionen, die Steuer und die Bewertung des Schadens steigen.

Durch die Handelsbeziehungen werden jedoch die Auswirkungen etwas abgemildert, da der Produktionsrückgang in Region 1 nicht vollkommen auf den Konsum durchschlägt, sondern die Region die nicht eingesetzten Faktoren auf dem Weltmarkt verkauft und im Gegenwert Konsumgüter kauft. 


\section{ZUSAMMENFASSUNG}

In diesem Kapitel werden die Modelle der Kapitel 4 und 5 zusammengefaßt. Es werden sowohl die direkten (Schadstoffdiffusion) als auch die indirekten Auswirkungen (Handelsbeziehungen) der interregionalen Verflechtung in einen Modellkontext integriert. Die Schadstoffdiffusion ist in einer Richtung (Unterlieger-Oberlieger-Problematik): Region 2 verschmutzt Region 1 mit. Die Ökononmie ist über Handelsbeziehungen am Weltmarkt beteiligt, sie ist jedoch ein kleines Land und kann deshalb die Höhe der Preise durch ihr Angebots- und Nachfrageverhalten nicht beeinflussen. Drei unterschiedliche institutionelle Arrangements werden betrachtet: Vollkommen autonome Regionen; Regionen, die von einer Zentralbehörde gesteuert werden; für die emissionsexportierende Region wird von einer Zentralbehörde ein bindender Emissionsstandard gesetzt.

Im Fall autonomer Regionalbehörden wird gezeigt, daß die emissionsimportierende Region ihre Umweltpolitik nach der emissionsexportierenden Region ausrichten muß, jedoch wird diese Abhängigkeit gegenüber dem Fall autarker Regionen [Kapitel 4] abgeschwächt, da diese Region die Möglichkeit hat, einen Teil der Konsumgüter nicht selbst zu produzieren, sondern sie von außerhalb zu beziehen.

Es wird gezeigt, daß die emissionsimportierende Region umso eher Faktoren exportiert, um dafür Konsumgüter zu kaufen, je höher die importierten Emissionen sind. Die Eigenschaften des Steady-States können folgendermaßen charakterisiert werden:

(i) Die Höhe der sozialen Zeitpräferenzrate und der Assimilationskapazitäten der Ökosysteme haben die analogen Effekte wie ohne Handelsbeziehungen [Kapitel 4].

(ii) Die Auswirkungen der Höhe der Faktorausstattungen werden entscheidend von den Handelsbeziehungen beeinflußt. Während es bei autarken Regionen für die emissionsimportierende Region vorteilhaft ist, wenn die emissionsexportierende Region eine "geringe" Faktorausstattung besitzt, ist mit Handelsbeziehungen der umgekehrte Fall gegeben. Da, je höher die Faktorausstattung in der emissionsexportie- 
renden Region 2 ist, dort umso weniger produziert wird, so daß auch die exportierten Emissionen sinken. Region 1 kann vermehrt ihr eigenes Ökosystem in Anspruch nehmen und mehr produzieren und auch konsumieren, während die Immissionen sinken.

(iii) Eine Relativpreiserhöhung hat auf die emissionsexportierende Region äquivalente Auswirkungen wie im Fall ohne Diffusion [Kapitel 5]. Für die emissionsimportierende Region 1 ändern sich jedoch durch den sinkenden Emissionsimport die Auswirkungen. Für Region 1 ist es weder hinreichend noch notwendig für eine Konsumsteigerung, daß sie Faktorexporteur ist, sondern durch die Emissionssenkung in der emissionsexportierenden Region ist trotz Ressourcenimports eine Konsumsteigerung möglich.

(iv) Die Auswirkungen einer Politik des hohen Schornsteins in Region 2 werden nicht entscheidend von den Handelsbeziehungen beeinflußt. Es ergeben sich die analogen Resultate zu den in Kapitel 4 erhaltenen.

Wird die Optimierung von einer Zentralregierung vorgenommen, ist das sich ergebende Steady-State wiederum Pareto-optimal. Auch hier ergibt sich, daß im Vergleich zur autonomen Optimierung die Emissionen in der emissionsexportierenden Region niedriger sind, während in der emissionsimportierenden Region mehr emittiert werden kann. Durch die sich ergebende wechselseitige Abhängigkeit, wird Region 2 gezwungen, die Schäden, die durch ihre Emissionstätigkeit in der anderen Region entstehen, zu berücksichtigen. Im einzelnen ergibt sich:

(i) Die Auswirkungen der Höhe der sozialen Zeitpräferenzrate, die Assimilationskapazität der Ökosysteme und des Diffusionskoeffizienten sind äquivalent zu denen einer autarken Ökonomie [Kapitel 4].

(ii) Durch die Güter- und Faktormobilität sind die Auswirkungen der Höhe der Faktorausstattungen genau entgegengesetzt zum autarken Fall des Kapitels 4. Insbesondere kann in der emissionsexportierenden Region umso mehr produziert werden, je höher die Faktorausstattung in der emissionsimportierenden Region ist. 
(iii) Für eine Relativpreiserhöhung können eindeutige Aussagen nur für die Immissionen, den Schattenpreis und die Steuer in Region 1 sowie die Steuer in Region 2 abgeleitet werden. Die Änderungsrichtungen der anderen Variablen bleiben weitgehend unbestimmt bzw. sind von mehreren Einflußfaktoren abhängig.

Das dritte institutionelle Arrangement autonomer Regionalbehörden mit einem Emissionsstandard für die emissionsexportierende Region wird hier ebenfalls untersucht:

(i) Wie auch schon bei autonomen Regionalbehörden und einer Zentralbehörde ergeben sich für die Höhe des Diffusionskoeffizienten, des Emissionsstandards, der Assimilationsfähigkeit des Ökosystems und der Diskontrate die äquivalenten Auswirkungen wie im autarken Fall.

(ii) Die Höhe der Faktorausstattung der Region 2 hat auf Region 1 keine Auswirkungen. Auf Region 2 hat die Höhe der Faktorausstattung in dieser Region jedoch wieder die durch die Handelsbeziehungen umgekehrten Vorzeichen wie im autarken Fall.

(iii) Die Auswirkungen durch eine Relativpreiserhöhung auf Region 1 sind vollkommen analog zu denjenigen ohne Diffusion. Auf Region 2 hat eine Erhöhung des Relativpreises die folgenden Auswirkungen:

- Die Steuer und die Kosten des Konsumverzichts sinken, während die Immissionen und der Schaden konstant bleiben.

- $\quad \mathrm{Ob}$ der Konsum in Region 2 sinkt oder steigt ist nur von der Handelsposition der Region abhängig.

Auch hier ergibt sich durch eine Emissionsstandardsetzung für die emissionsexportierende Region eine Verstetigung der Politik für beide Regionen. Durch die Handelsbeziehungen der Regionen ist für Region 1, der emissionsimportierenden Region, darüberhinaus auch eine Abschwächung der Abhängigkeit von der in Region 2 betriebenen Umweltpolitik, z. B. bei einer Erhöhung des Diffusionskoeffizienten, gegeben. 


\section{ANHANG 6.I: Autonome Regionalregierungen}

\section{Die Änderungen für Region 2}

Folgendes Gleichungssystem wird total differenziert:

$$
\begin{aligned}
\left(1-\gamma_{2}\right) E_{2}-\alpha_{2} S_{2} & =0 \\
\left(\delta+\alpha_{2}\right) p_{2}-u^{2}{ }_{S} & =0 \\
\tau_{2}+\left(1-\gamma_{2}\right) p_{2} & =0 \\
E_{2}-E^{2}\left(\tau_{2}\right) & =0
\end{aligned}
$$

Man erhält

$$
\begin{aligned}
& {\left[\begin{array}{cccc}
1-\gamma_{2} & -\alpha_{2} & 0 & 0 \\
0 & -u_{S S}^{2} & \delta+\alpha_{2} & 0 \\
0 & 0 & 1-\gamma_{2} & 1 \\
1 & 0 & 0 & -E^{2} \tau
\end{array}\right]\left[\begin{array}{l}
d E_{2} \\
d S_{2} \\
d p_{2} \\
d \tau_{2}
\end{array}\right]=\left[\begin{array}{l}
0 \\
-p_{2} \\
0 \\
0
\end{array}\right] d \delta+\left[\begin{array}{l}
S_{2} \\
-p_{2} \\
0 \\
0
\end{array}\right] d \alpha_{2}+} \\
& +\left[\begin{array}{c}
0 \\
0 \\
0 \\
E_{\mathrm{R}}^{2}
\end{array}\right] d \mathbf{R}_{2}+\left[\begin{array}{c}
E_{2} \\
0 \\
p_{2} \\
0
\end{array}\right] d \gamma_{2}+\left[\begin{array}{c}
0 \\
0 \\
0 \\
E_{\mathrm{q}}^{2}
\end{array}\right] d \mathbf{q}
\end{aligned}
$$

Die Systemdeterminante ist

$$
\Delta_{2}=\left(1-\gamma_{2}\right)^{2} u_{S S}^{2} E^{2}{ }_{\tau}+\alpha_{2}\left(\delta+\alpha_{2}\right)>0
$$


Ein Vergleich mit dem Gleichungssystem in Anhang 4.I zeigt, daß hier ein formal weitgehend äquivalentes Gleichungssystem untersucht wird. $\mathrm{Zu}$ sätzlich werden die Auswirkungen einer Relativpreiserhöhung diskutiert. Weiterhin ist zu beachten, daß sich das Vorzeichen von $E^{\imath}{ }_{\mathbf{R}}$ ändert. Die Ableitung dieses Vorzeichens sowie die Definition von $E_{\mathrm{q}}^{i}$ wird in Anhang 5 gegeben.

1.1 Die Änderungen für ein erhöhtes $\delta$ :

$$
\begin{aligned}
& \frac{d E_{2}}{d \delta}=\frac{\left(1-\mathrm{Y}_{2}\right) \mathrm{a}_{2} E_{\mathrm{L}}^{2} p_{2}}{\Delta_{2}}>0 \\
& \frac{d S_{2}}{d \delta}=\frac{\left(1-\mathrm{Y}_{2}\right)^{2} E_{\tau}^{2} p_{2}}{\Delta_{2}}>0 \\
& \frac{d p_{2}}{d \delta}=\frac{-\mathrm{a}_{2} p_{2}}{\Delta_{2}}>0 \\
& \frac{d \tau_{2}}{d \delta}=\frac{\left(1-\mathrm{Y}_{2}\right) \mathrm{a}_{2} p_{2}}{\Delta_{2}}<0 \\
& \frac{d C_{2}}{d \delta}=\frac{C_{E}^{2}\left(1-\mathrm{Y}_{2}\right) \mathrm{a}_{2} E_{\mathrm{t}}^{2} p_{2}}{\Delta_{2}}>0
\end{aligned}
$$

1.2 Die Änderungen für ein erhöhtes $\alpha_{2}$ :

$$
\begin{aligned}
& \frac{d E_{2}}{d a_{2}}=\frac{\left(1-\mathrm{Y}_{2}\right)^{2} E_{\imath}^{2}\left|u_{S S}^{2} S_{2}+\mathrm{a}_{2} p_{2}\right|}{\Delta_{2}}>0 \\
& \frac{d S_{2}}{d a_{2}}=\frac{\left(1-\mathrm{Y}_{2}\right)^{2} E_{\mathrm{t}}^{2} p_{2}-\left(\delta+\mathrm{a}_{2}\right) S_{2}}{\Delta_{2}} \geq_{<}
\end{aligned}
$$




$$
\begin{aligned}
& \frac{d p_{2}}{d \mathrm{a}_{2}}=\frac{-\left[u_{\left.S S_{2}^{2} S_{2}+\mathrm{a}_{2} p_{2}\right]}\right.}{\Delta_{2}}>0 \\
& \frac{d \mathrm{\tau}_{2}}{d \mathrm{a}_{2}}=\frac{\left(1-\mathrm{v}_{2}\right)\left[u_{S S_{2}}^{2} S_{2}+\mathrm{a}_{2} p_{2}\right]}{\Delta_{2}}<0 \\
& \frac{d C_{2}}{d \mathrm{a}_{2}}=\frac{C_{E}^{2}\left(1-\mathrm{v}_{2}\right) E_{\mathrm{t}}^{2}\left[u_{S S^{2}}^{2} S_{2}+\mathrm{a}_{2} p_{2}\right]}{\Delta_{2}}>0
\end{aligned}
$$

1.3 Die Änderungen für ein erhöhtes $\mathbf{R}_{2}$ :

$$
\begin{aligned}
& \frac{d E_{2}}{d \mathbf{R}_{2}}=\frac{a_{2}\left(\delta+a_{2}\right) E_{\mathbf{R}}^{2}}{\Delta_{2}}<0 \\
& \frac{d S_{2}}{d \mathbf{R}_{2}}=\frac{\left(1-\mathrm{Y}_{2}\right)\left(\delta+\mathrm{a}_{2}\right) E_{\mathbf{R}}^{2}}{\Delta_{2}}<0 \\
& \frac{d p_{2}}{d \mathbf{R}_{2}}=\frac{\left(1-\mathrm{Y}_{2}\right) u_{S S}^{2} E_{\mathbf{R}}^{2}}{\Delta_{2}}>0 \\
& \frac{d \tau_{2}}{d \mathbf{R}_{2}}=\frac{-\left(1-\mathrm{Y}_{2}\right)^{2} u_{S S}^{2} E_{\mathbf{R}}^{2}}{\Delta_{2}}<0 \\
& \frac{d C_{2}}{d \mathbf{R}_{2}}=\frac{\mathrm{q} E_{\mathrm{t}}^{2}}{\Delta_{2}}\left\{\left(1-\mathrm{Y}_{2}\right)^{2} u_{S S}^{2}+\mathrm{a}_{2}\left(\delta+\mathrm{a}_{2}\right) u_{C}^{2} C_{E E}^{2}\right\}>0
\end{aligned}
$$

1.4 Die Änderungen für ein erhöhtes q:

$$
\frac{d E_{2}}{d q}=\frac{a_{2}\left(\delta+a_{2}\right) E_{q}^{2}}{\Delta_{2}}<0
$$




$$
\begin{aligned}
& \frac{d S_{2}}{d \mathbf{q}}=\frac{\left(1-\mathrm{y}_{2}\right)\left(\delta+\mathrm{a}_{2}\right) E_{\mathrm{q}}^{2}}{\Delta_{2}}<0 \\
& \frac{d p_{2}}{d \mathbf{q}}=\frac{\left(1-\mathrm{Y}_{2}\right) u_{S S}^{2} E_{\mathrm{q}}^{2}}{\Delta_{2}}>0 \\
& \frac{d \tau_{2}}{d \mathbf{q}}=\frac{-\left(1-\mathrm{Y}_{2}\right)^{2} u_{S S}^{2} E_{\mathrm{q}}^{2}}{\Delta_{2}}<0 \\
& \frac{d C_{2}}{d \mathbf{q}}=\frac{E_{\tau}^{2}}{\Delta_{2}}\left\{\left(1-\mathrm{Y}_{2}\right)^{2} u_{S S}^{2} M_{2}-\mathrm{a}_{2}\left(\delta+\mathrm{a}_{2}\right) u_{C}^{2}\left(C_{E E}^{2} M_{2}+C_{E \mathbf{q}}^{2} C_{E}^{2}\right)\right\} \geq_{0}
\end{aligned}
$$

\subsection{Die Änderungsrichtungen für ein erhöhtes $\gamma_{2}$ :}

$$
\begin{aligned}
& \frac{d E_{2}}{d \mathrm{Y}_{2}}=\frac{E_{\tau}^{2}\left[\left(1-\mathrm{Y}_{2}\right) u_{S S}^{2} E_{2}+a_{2}\left(\delta+a_{2}\right) p_{2}\right]}{\Delta_{2}}>0 \\
& \frac{d S_{2}}{d \mathrm{Y}_{2}}=\frac{\left(\delta+a_{2}\right)\left[\left(1-\mathrm{Y}_{2}\right) E_{\tau}^{2} p_{2}-E_{2}\right]}{\Delta_{2}} \geq_{0} \\
& \frac{d p_{2}}{d \mathrm{Y}_{2}}=\frac{u_{S S}^{2}\left[\left(1-\mathrm{Y}_{2}\right) E_{\tau}^{2} p_{2}-E_{2}\right]}{\Delta_{2}} \geq_{0} \\
& \frac{d \tau_{2}}{d \mathrm{Y}_{2}}=\frac{\left(1-\mathrm{Y}_{2}\right) u_{S S}^{2} E_{2}+a_{2}\left(\delta+a_{2}\right) p_{2}}{\Delta_{2}}<0 \\
& \frac{d C_{2}}{d \mathrm{Y}_{2}}=\frac{C_{E}^{2} E_{\tau}^{2}\left[\left(1-\mathrm{Y}_{2}\right) u_{S S_{2}}^{2} E_{2}+a_{2}\left(\delta+a_{2}\right) p_{2}\right]}{\Delta_{2}}>0
\end{aligned}
$$




\section{$\underline{\text { 2. Die Änderungen für Region } 1}$}

Für Region 1 erhalten wir ein analoges Gleichungssystem, bei dem allerdings die Abhängigkeit von Region 2 mit berücksichtigt werden muß:

$$
\begin{aligned}
E_{1}-\alpha_{1} S_{1} & =-\gamma_{2} E_{2} \\
\left(\delta+\alpha_{1}\right) p_{1}-u_{S}^{1} & =0 \\
\tau_{1}+p_{1} & =0 \\
E_{1}-E^{1}\left(\tau_{1}\right) & =0
\end{aligned}
$$

Totale Differentation ergibt

$$
\begin{gathered}
{\left[\begin{array}{cccc}
1 & -\alpha_{1} & 0 & 0 \\
0 & -u_{S S}^{1} & \delta \alpha_{1} & 0 \\
0 & 0 & 1 & 1 \\
1 & 0 & 0 & -E_{\tau}^{1}
\end{array}\right]\left[\begin{array}{c}
d E_{1} \\
d S_{1} \\
d p_{1} \\
d \tau_{1}
\end{array}\right]=\left[\begin{array}{c}
\gamma_{2} E^{2^{*}} \delta \\
-p_{1} \\
0 \\
0
\end{array}\right] d \delta+\left[\begin{array}{c}
S_{1} \\
-p_{1} \\
0 \\
0
\end{array}\right] d \alpha_{1}+} \\
+\left[\begin{array}{c}
0 \\
0 \\
0 \\
E^{1}{ }_{R}
\end{array}\right] d \mathbf{R}_{1}+\left[\begin{array}{c}
\gamma_{2} E^{2^{*}} \\
0 \\
0 \\
0
\end{array}\right] d z+\left[\begin{array}{c}
\mathbf{e}_{2} \\
0 \\
0 \\
0
\end{array}\right] d \gamma_{2}+\left[\begin{array}{c}
\gamma_{2} E^{2^{*}} \\
0 \\
0 \\
E_{\mathbf{q}}^{1}
\end{array}\right] d \mathbf{q}
\end{gathered}
$$

mit $e_{2}=-\gamma_{2} E^{2^{*}}{ }_{\gamma}-E_{2}<0$,

$$
z=\alpha_{2}, \mathbf{R}_{2}
$$

Die Systemdeterminante ist

$$
\Delta_{1}=u_{S S^{1}}^{E^{1}} \tau+\alpha_{1}\left(\delta+\alpha_{1}\right)>0
$$


2.1 Die Änderungen für ein erhöhtes $\delta$ :

$$
\begin{aligned}
& \frac{d E_{1}}{d \delta}=\frac{E_{\tau}^{1}\left[a_{1} p_{1}-Y_{2} E_{\delta}^{2^{*}} u_{S S}^{1}\right]}{\Delta_{1}} \underset{<0}{\geq} \\
& \frac{d S_{1}}{d \delta}=\frac{E_{\imath}^{1} p_{1}+Y_{2}\left(\delta+a_{1}\right) E_{\delta}^{2^{\star}}}{\Delta_{1}}>0 \\
& \frac{d p_{1}}{d \delta}=\frac{Y_{2} E_{\delta}^{2 *} u_{S S}^{1}-a_{1} p_{1}}{\Delta_{1}} \geq_{0} \\
& \frac{d \tau_{1}}{d \delta}=\frac{a_{1} p_{1}-Y_{2} E_{\delta}^{2^{*}} u_{S S}^{1}}{\Delta_{1}} \geq_{0} \\
& \frac{d C_{1}}{d \delta}=\frac{C_{E}^{1} E_{\mathrm{r}}^{1}\left[a_{1} p_{1}-v_{2} E_{\delta}^{2 *} u_{S S}^{1}\right]}{\Delta_{1}} \geq 0
\end{aligned}
$$

2.2 Die Änderungen für ein erhöhtes $\alpha_{1}$ :

$$
\begin{aligned}
& \frac{d E_{1}}{d a_{1}}=\frac{E_{\tau}^{1}\left[u_{S S}^{1} S_{1}+a_{1} p_{1}\right]}{\Delta_{1}}>0 \\
& \frac{d S_{1}}{d a_{1}}=\frac{E_{\tau}^{1} p_{1}-\left(\delta+a_{1}\right) S_{1}}{\Delta_{1}} \geq_{0} \\
& \frac{d p_{1}}{d a_{1}}=\frac{-\left[u_{S S}^{1} S_{1}+a_{1} p_{1}\right]}{\Delta_{1}}>0 \\
& \frac{d \tau_{1}}{d a_{1}}=\frac{u_{S S}^{1} S_{1}+a_{1} p_{1}}{\Delta_{1}}<0
\end{aligned}
$$




$$
\frac{d C_{1}}{d a_{1}}=\frac{C_{E}^{1} E_{\tau}^{1}\left[u_{S S}^{1} S_{1}+a_{1} p_{1}\right]}{\Delta_{1}}>0
$$

2.3 Die Änderungen für ein erhöhtes $\mathbf{R}_{1}$ :

$$
\begin{aligned}
& \frac{d E_{1}}{d \mathbf{R}_{1}}=\frac{a_{1}\left(\delta+a_{1}\right) E_{\mathbf{R}}^{1}}{\Delta_{1}}<0 \\
& \frac{d S_{1}}{d \mathbf{R}_{1}}=\frac{\left(\delta+a_{1}\right) E_{\mathbf{R}}^{1}}{\Delta_{1}}<0 \\
& \frac{d p_{1}}{d \mathbf{R}_{1}}=\frac{u_{S S}^{1} E_{\mathbf{R}}^{1}}{\Delta_{1}}>0 \\
& \frac{d \tau_{1}}{d \mathbf{R}_{1}}=-\frac{u_{S S}^{1} E_{\mathbf{R}}^{1}}{\Delta_{1}}>0 \\
& \frac{d C_{1}}{d \mathbf{R}_{1}}=\frac{a_{1}\left(\delta+a_{1}\right) E_{\mathbf{R}}^{1}}{\Delta_{1}}<0
\end{aligned}
$$

2.4 Die Änderungen in Region 1 bei erhöhtem $\alpha_{2}$ :

Es gilt $\quad E^{2^{*}}{ }_{\alpha}=d E_{2}{ }^{\infty} / d \alpha_{2}>0$

$$
\begin{aligned}
& \frac{d E_{1}}{d a_{2}}=\frac{-Y_{2} E_{\tau}^{1} u_{S S}^{1} E_{a}^{2^{*}}}{\Delta_{1}}<0 \\
& \frac{d S_{1}}{d a_{2}}=\frac{Y_{2}\left(\delta+a_{1}\right) E_{a}^{2^{*}}}{\Delta_{1}}>0 \\
& \frac{d p_{1}}{d a_{2}}=\frac{Y_{2} u_{S S}^{1} E_{a}^{2 *}}{\Delta_{1}}<0
\end{aligned}
$$




$$
\begin{aligned}
& \frac{d \tau_{1}}{d a_{2}}=-\frac{Y_{2} u_{S S}^{1} E_{a}^{2^{*}}}{\Delta_{1}}>0 \\
& \frac{d C_{1}}{d a_{2}}=\frac{-C_{E}^{1} Y_{2} E_{\tau}^{1} u_{S S}^{1} E_{a}^{2 *}}{\Delta_{1}}<0
\end{aligned}
$$

2.5 Die Änderungen in Region 1 bei erhöhtem $\mathbf{R}_{2}$ :

Es gilt $\quad E^{2^{*}}<0$

$$
\begin{aligned}
& \frac{d E_{1}}{d \mathbf{R}_{2}}=\frac{-\mathrm{Y}_{2} E_{\mathrm{\tau}}^{1} u_{S S}^{1} E_{\mathrm{R}}^{2^{*}}}{\Delta_{1}}>0 \\
& \frac{d S_{1}}{d \mathbf{R}_{2}}=\frac{Y_{2}\left(\delta+a_{1}\right) E_{\mathbf{R}}^{2^{*}}}{\Delta_{1}}<0 \\
& \frac{d p_{1}}{d \mathbf{R}_{2}}=\frac{\mathrm{Y}_{2} u_{S S}^{1} E_{\mathbf{R}}^{2^{*}}}{\Delta_{1}}>0 \\
& \frac{d \tau_{1}}{d \mathbf{R}_{2}}=-\frac{Y_{2} u_{S S}^{1} E_{\mathbf{R}}^{2^{*}}}{\Delta_{1}}<0 \\
& \frac{d C_{1}}{d \mathbf{R}_{2}}=\frac{-C_{E}^{1} \mathrm{Y}_{2} E_{\tau}^{1} u_{S S}^{1} E_{\mathbf{R}}^{2 *}}{\Delta_{1}}>0
\end{aligned}
$$

2.6 Die Änderungen in Region 1 bei erhöhtem q:

Es gilt $\quad E^{2^{*}}<0$

$$
\begin{aligned}
& \frac{d E_{1}}{d \mathrm{q}}=\frac{a_{1}\left(\delta+a_{1}\right) E_{\mathrm{q}}^{1}-\mathrm{Y}_{2} E_{\mathrm{\tau}}^{1} u_{S S}^{1} E_{\mathrm{q}}^{2^{*}}}{\Delta_{1}} \geq_{0} \\
& \frac{d S_{1}}{d \mathrm{q}}=\frac{\left(\delta+a_{1}\right)}{\Delta_{1}}\left|E_{\mathrm{q}}^{1}+\mathrm{Y}_{2} E_{\mathrm{q}}^{2^{*}}\right|<0
\end{aligned}
$$




$$
\begin{aligned}
& \frac{d p_{1}}{d \mathrm{q}}=\frac{u_{S S}^{1}}{\Delta_{1}}\left|E_{\mathrm{q}}^{1}+\mathrm{Y}_{2} E_{\mathrm{q}}^{2^{*}}\right|>0 \\
& \left.\frac{d \tau_{1}}{d \mathrm{q}}=-\frac{u_{S S}^{1}}{\Delta_{1}} \mid E_{\mathrm{q}}^{1}+\mathrm{Y}_{2} E_{\mathrm{q}}^{2^{*}}\right]<0 \\
& \frac{d C_{1}}{d \mathrm{q}}=\frac{-E_{\tau}^{1}}{\Delta_{1}}\left\{\mathrm{Y}_{2} C_{E}^{1} E_{\mathrm{q}}^{2^{*}} u_{S S}^{1}-u_{S S}^{1} C_{\mathrm{q}}^{1}+\mathrm{a}_{1}\left(\delta+\mathrm{a}_{1}\right) u_{C}^{1}\left[C_{E}^{1} C_{E \mathrm{q}}^{1}-C_{E E}^{1} C_{\mathrm{q}}^{1} \mid\right\} \geq 0\right.
\end{aligned}
$$

\subsection{Die Änderungen für ein größeres $\gamma_{2}$ :}

$$
\begin{aligned}
& \frac{d E_{1}}{d Y_{2}}=\frac{E_{\tau}^{1} u_{S S}^{1} e_{2}}{\Delta_{1}}<0 \\
& \frac{d S_{1}}{d Y_{2}}=\frac{-\left(\delta+a_{1}\right) \mathbf{e}_{2}}{\Delta_{1}}>0 \\
& \frac{d p_{1}}{d Y_{2}}=\frac{-u_{s s}^{1} e_{2}}{\Delta_{1}}<0 \\
& \frac{d \tau_{1}}{d Y_{2}}=\frac{u_{S S^{1}}^{1} \mathrm{e}_{2}}{\Delta_{1}}>0 \\
& \frac{d C_{1}}{d Y_{2}}=\frac{C_{E}^{1} E_{\tau}^{1} u_{S s}^{1} e_{2}}{\Delta_{1}}<0
\end{aligned}
$$




\section{Anhang 6.II: Zentralregierung}

Für den Zentralregierungsfall können nicht mehr zwei unabhängige Systeme betrachtet werden, sondern es ist ein interdependentes System zu analysieren:

$$
\begin{aligned}
E_{1}-\alpha_{1} S_{1}+\gamma_{2} E_{2} & =0 \\
\left(\delta+\alpha_{1}\right) p_{1}-u^{1}{ }_{S} & =0 \\
\tau_{1}+p_{1} & =0 \\
E_{1}-E^{1}\left(\tau_{1}\right) & =0 \\
\left(1-\gamma_{2}\right) E_{2}-\alpha_{2} S_{2} & =0 \\
\left(\delta+\alpha_{2}\right) p_{2}-u_{S}^{2} & =0 \\
\tau_{2}+\left(1-\gamma_{2}\right) p_{2}+\gamma_{2} p_{1} & =0 \\
E_{2}-E^{2}\left(\tau_{2}\right) & =0
\end{aligned}
$$

Totale Differentiation ergibt

$\left[\begin{array}{cccccccc}1 & -\alpha_{1} & 0 & 0 & \gamma_{2} & 0 & 0 & 0 \\ 0 & -u_{S S}^{1} & \delta+\alpha_{1} & 0 & 0 & 0 & 0 & 0 \\ 0 & 0 & 1 & 1 & 0 & 0 & 0 & 0 \\ 1 & 0 & 0 & -E^{1} \tau & 0 & 0 & 0 & 0 \\ 0 & 0 & 0 & 0 & 1-\gamma_{2} & -\alpha_{2} & 0 & 0 \\ 0 & 0 & 0 & 0 & 0 & -u_{S S}^{2} & \delta+\alpha_{2} & 0 \\ 0 & 0 & \gamma_{2} & 0 & 0 & 0 & 1-\gamma_{2} & 1 \\ 0 & 0 & 0 & 0 & 1 & 0 & 0 & -E^{2} \tau\end{array}\right]\left[\begin{array}{l}d E_{1} \\ d S_{1} \\ d p_{1} \\ d \tau_{1} \\ d E_{2} \\ d S_{2} \\ d p_{2} \\ d \tau_{2}\end{array}\right]$




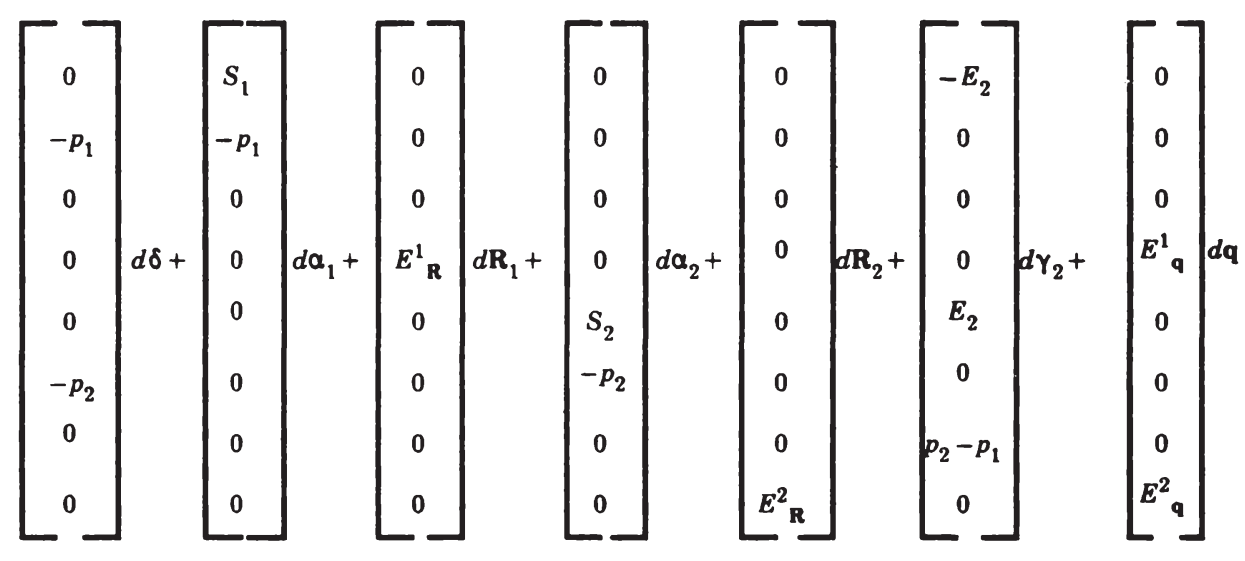

Die Systemdeterminante ist

$$
\begin{aligned}
& \Delta=\left[u_{S S}^{1} E^{1} \tau+\alpha_{1}\left(\delta+\alpha_{1}\right)\right]\left[\left(1-\gamma_{2}\right)^{2} u^{2}{ }_{S S} E^{2}{ }_{\tau}+\alpha_{2}\left(\delta+\alpha_{2}\right)\right]+ \\
& \gamma_{2}{ }^{2} \alpha_{2}\left(\delta+\alpha_{2}\right) u_{S S}^{1} E_{\tau}^{2}>0
\end{aligned}
$$

1. Die Änderungen für ein erhöhtes $\alpha_{1}$ :

$$
\begin{aligned}
& \frac{d E_{1}}{d a_{1}}=\frac{E_{\tau}^{1}\left[u_{S S}^{1} S_{1}+a_{1} p_{1}\right]\left[\left(1-Y_{2}\right)^{2} u_{S S}^{2} E_{\tau}^{2}+a_{2}\left(\delta+a_{2}\right)\right]}{\Delta}>0 \\
& \frac{d S_{1}}{d a_{1}}=\frac{\left[p_{1} E_{\tau}^{1}-\left(\delta+a_{1}\right) S_{1}\right]\left[\left(1-Y_{2}\right)^{2} u_{S S}^{2} E_{\tau}^{2}+a_{2}\left(\delta+a_{2}\right)\right]+a_{2}\left(\delta+a_{2}\right) Y_{2}^{2} E_{\tau}^{2} p_{1}}{\Delta} \geq 0 \\
& \frac{d p_{1}}{d a_{1}}=\frac{-\left[u_{S S}^{1} S_{1}+a_{1} p_{1}\right]\left[\left(1-Y_{2}\right)^{2} u_{S S}^{2} E_{\tau}^{2}+a_{2}\left(\delta+a_{2}\right)\right]}{\Delta}>0 \\
& \frac{d \tau_{1}}{d a_{1}}=\frac{\left[u_{S S}^{1} S_{1}+a_{1} p_{1}\right]\left[\left(1-Y_{2}\right)^{2} u_{S S}^{2} E_{\tau}^{2}+a_{2}\left(\delta+a_{2}\right)\right]}{\Delta}<0
\end{aligned}
$$


$\frac{d C_{1}}{d a_{1}}=\frac{C_{E}^{1} E_{\tau}^{1}\left[u_{S S}^{1} S_{1}+a_{1} p_{1}\right]\left[\left(1-Y_{2}\right)^{2} u_{S S}^{2} E_{\mathrm{t}}^{2}+\mathrm{a}_{2}\left(\delta+\mathrm{a}_{2}\right)\right]}{\Delta}>0$

$\frac{d E_{2}}{d a_{1}}=\frac{a_{2}\left(\delta+a_{2}\right) \mathrm{Y}_{2} E_{\tau}^{2}\left[u_{S S}^{1} S_{1}+a_{1} p_{1}\right]}{\Delta}>0$

$\frac{d S_{2}}{d a_{1}}=\frac{\left(\delta+a_{2}\right)\left(1-Y_{2}\right) y_{2} E_{t}^{2}\left[u_{S S}^{1} S_{1}+a_{1} p_{1}\right]}{\Delta}>0$

$\frac{d p_{2}}{d a_{1}}=\frac{\left(1-Y_{2}\right) Y_{2} E_{\tau}^{2} u_{S S}^{2}\left|u_{S S}^{1} S_{1}+a_{1} p_{1}\right|}{\Delta}<0$

$\frac{d \tau_{2}}{d a_{1}}=\frac{a_{2}\left(\delta+a_{2}\right) Y_{2}\left[u_{S S}^{1} S_{1}+a_{1} p_{1}\right]}{\Delta}<0$

$\frac{d C_{2}}{d a_{1}}=\frac{a_{2}\left(\delta+a_{2}\right) C_{E}^{2} Y_{2} E_{\tau}^{2}\left[u_{S S}^{1} S_{1}+a_{1} p_{1}\right]}{\Delta}>0$

2. Die Änderungen für ein erhöhtes $\alpha_{2}$ :

$\frac{d E_{1}}{d a_{2}}=\frac{-Y_{2}\left(1-Y_{2}\right) u_{S S}^{1} E_{c}^{1} E_{t}^{2}\left|u_{S S}^{2} S_{2}+a_{2} p_{2}\right|}{\Delta}<0$

$\frac{d S_{1}}{d a_{2}}=\frac{\left(\delta+a_{1}\right) Y_{2}\left(1-y_{2}\right) E_{\tau}^{2}\left[u_{S S}^{2} S_{2}+a_{2} p_{2}\right]}{\Delta}>0$

$\frac{d p_{1}}{d a_{2}}=\frac{u_{S S}^{1} Y_{2}\left(1-Y_{2}\right) E_{\tau}^{2}\left[u_{S S}^{2} S_{2}+a_{2} p_{2}\right]}{\Delta}<0$ 


$$
\begin{aligned}
& \frac{d \tau_{1}}{d \mathrm{a}_{2}}=-\frac{u_{S S}^{1} \mathrm{Y}_{2}\left(1-\mathrm{Y}_{2}\right) E_{\mathrm{\tau}}^{2}\left|u_{S S}^{2} S_{2}+\mathrm{a}_{2} p_{2}\right|}{\Delta}>0 \\
& \frac{d E_{2}}{d \mathrm{a}_{2}}=\frac{\left(1-\mathrm{Y}_{2}\right) E_{\mathrm{t}}^{2}\left[u_{S S}^{1} E_{\mathrm{t}}^{1}+\mathrm{a}_{1}\left(\delta+\mathrm{a}_{1}\right)\right]\left[u_{S S}^{2} S_{2}+\mathrm{a}_{2} p_{2}\right]}{\Delta}>0 \\
& \frac{d C_{1}}{d a_{2}}=\frac{-Y_{2}\left(1-Y_{2}\right) C_{E}^{1} u_{S S}^{1} E_{\mathrm{r}}^{1} E_{\mathrm{r}}^{2}\left[u_{S S}^{2} S_{2}+\mathrm{a}_{2} p_{2}\right]}{\Delta}<0
\end{aligned}
$$

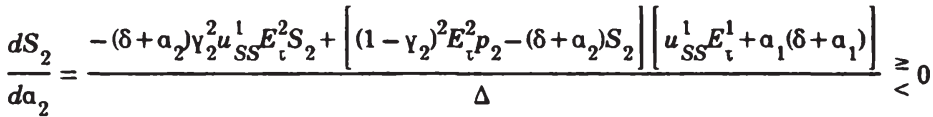

$$
\begin{aligned}
& \frac{d p_{2}}{d a_{2}}=\frac{-\left|u_{S S}^{1}\left(E_{\tau}^{1}+\mathrm{Y}_{2}^{2} E_{\tau}^{2}\right)+a_{1}\left(\delta+a_{1}\right)\right|\left|u_{S S}^{2} S_{2}+a_{2} p_{2}\right|}{\Delta}>0 \\
& \frac{d \tau_{2}}{d \mathrm{a}_{2}}=\frac{\left(1-\mathrm{Y}_{2}\right)\left[u_{S S}^{1} E_{\mathrm{t}}^{1}+a_{1}\left(\delta+a_{1}\right) \mid\left[u_{S S}^{2} S_{2}+a_{2} p_{2}\right]\right.}{\Delta}<0 \\
& \frac{d C_{2}}{d \mathrm{a}_{2}}=\frac{\left(1-\mathrm{Y}_{2}\right) C_{E}^{2} E_{\tau}^{2}\left[u_{S S}^{1} E_{\tau}^{1}+a_{1}\left(\delta+a_{1}\right) \mid\left[u_{S S}^{2} S_{2}+a_{2} p_{2}\right]\right.}{\Delta}>0
\end{aligned}
$$

\section{Die Änderungen für ein erhöhtes $\delta$ :}

$$
\frac{d E_{1}}{d \delta}=\frac{E_{\tau}^{1}\left\{a_{1} p_{1}\left[\left(1-Y_{2}\right)^{2} u_{S S}^{2} E_{\tau}^{2}+a_{2}\left(\delta+a_{2}\right)\right]-a_{2} p_{2} Y_{2}\left(1-Y_{2}\right) u_{S S}^{1} E_{\tau}^{2}\right\}}{\Delta} \geq 0
$$


$\frac{d S_{1}}{d \delta}=\frac{E_{\mathrm{t}}^{1} p_{1}\left[\left(1-\mathrm{Y}_{2}\right)^{2} u_{S S}^{2} E_{\mathrm{t}}^{2}+\mathrm{a}_{2}\left(\delta+\mathrm{a}_{2}\right)\right]+\mathrm{a}_{2} \mathrm{Y}_{2} E_{\mathrm{t}}^{2}\left(\mathrm{Y}_{2}\left(\delta+\mathrm{a}_{2}\right) p_{1}+\left(1-\mathrm{Y}_{2}\right)\left(\delta+\mathrm{a}_{1}\right) p_{2}\right)}{\Delta}>0$

$\frac{d p_{1}}{d \delta}=-\frac{a_{1} p_{1}\left[\left(1-Y_{2}\right)^{2} u_{S S}^{2} E_{\tau}^{2}+a_{2}\left(\delta+a_{2}\right)\right]-a_{2} p_{2} Y_{2}\left(1-Y_{2}\right) u_{S S}^{1} E_{\tau}^{2}}{\Delta} \geq 0$

$\frac{d \tau_{1}}{d \delta}=\frac{a_{1} p_{1}\left[\left(1-\mathrm{Y}_{2}\right)^{2} u_{S S}^{2} E_{\tau}^{2}+a_{2}\left(\delta+a_{2}\right)\right]-a_{2} p_{2} Y_{2}\left(1-Y_{2}\right) u_{S S}^{1} E_{\mathrm{r}}^{2}}{\Delta} \underset{<0}{\geq}$

$\frac{d C_{1}}{d \delta}=\frac{C_{E}^{1} E_{\tau}^{1}\left\{a_{1} p_{1}\left[\left(1-Y_{2}\right)^{2} u_{S S}^{2} E_{\tau}^{2}+a_{2}\left(\delta+a_{2}\right)\right]-a_{2} p_{2} Y_{2}\left(1-Y_{2}\right) u_{S S}^{1} E_{\tau}^{2}\right\}}{\Delta} \underset{<0}{\geq}$

$\frac{d E_{2}}{d \delta}=\frac{a_{2} E_{\tau}^{2}\left\{Y_{2} a_{1}\left(\delta+a_{2}\right) p_{1}+\left(1-Y_{2}\right) p_{2}\left[u_{S S}^{1} E_{\tau}^{1}+a_{1}\left(\delta+a_{1}\right)\right]\right\}}{\Delta}>0$

$\frac{d S_{2}}{d \delta}=\frac{Y_{2}\left(1-Y_{2}\right) a_{1}\left(\delta+a_{2}\right) p_{1} E_{\tau}^{2}+\left(1-Y_{2}\right)^{2} p_{2} E_{\tau}^{2}\left[u_{S S}^{1} E_{\tau}^{1}+a_{1}\left(\delta+a_{1}\right)\right]}{\Delta}>0$

$\frac{d p_{2}}{d \delta}=\frac{\mathrm{Y}_{2}\left(1-\mathrm{Y}_{2}\right) \mathrm{a}_{1} u_{S S_{1}}^{2} E_{\mathrm{\tau}}-\mathrm{a}_{2} p_{2}\left[\mathrm{Y}_{2}^{2} u_{S S}^{1} E_{\tau}^{2}+u_{S S}^{1} E_{\tau}^{1}+\mathrm{a}_{1}\left(\delta+\mathrm{a}_{1}\right)\right]}{\Delta} \geq 0$

$\frac{d \tau_{2}}{d \delta}=\frac{a_{2}\left\{Y_{2} a_{1}\left(\delta+a_{2}\right) p_{1}+\left(1-Y_{2}\right) p_{2}\left[u_{S S}^{1} E_{\tau}^{1}+a_{1}\left(\delta+a_{1}\right)\right]\right\}}{\Delta}<0$

$\frac{d C_{2}}{d \delta}=\frac{a_{2} C_{E}^{2} E_{\tau}^{2}\left\{Y_{2} a_{1}\left(\delta+a_{2}\right) p_{1}+\left(1-Y_{2}\right) p_{2}\left[u_{S S}^{1} E_{\tau}^{1}+a_{1}\left(\delta+a_{1}\right)\right]\right\}}{\Delta}>0$ 
4. Die Änderungen für ein erhöhtes $\mathbf{R}_{1}$ :

$$
\begin{aligned}
& \frac{d E_{1}}{d \mathbf{R}_{1}}=\frac{\left.E_{\mathbf{R}}^{1}\left\{\mathrm{a}_{1}\left(\delta+\mathrm{a}_{1}\right) \mid\left(1-\mathrm{y}_{2}\right)^{2} u_{S S}^{2} E_{\mathrm{t}}^{2}+\mathrm{a}_{2}\left(\delta+\mathrm{a}_{2}\right)\right]+\mathrm{a}_{2}\left(\delta+\mathrm{a}_{2}\right) \mathrm{Y}_{2}^{2} u_{S S}^{1} E_{\mathrm{r}}^{2}\right\}}{\Delta}<0 \\
& \frac{d S_{1}}{d \mathbf{R}_{1}}=\frac{E_{\mathbf{R}}^{1}\left(\delta+\mathrm{a}_{1}\right)\left[\left(1-\mathrm{v}_{2}\right)^{2} u_{S S}^{2} E_{\tau}^{2}+\mathrm{a}_{2}\left(\delta+\mathrm{a}_{2}\right)\right]}{\Delta}<0 \\
& \frac{d p_{1}}{d \mathbf{R}_{1}}=\frac{u_{S S}^{1} E_{\mathbf{R}}^{1}\left[\left(1-\mathrm{Y}_{2}\right)^{2} u_{S S}^{2} E_{\tau}^{2}+\mathrm{a}_{2}\left(\delta+\mathrm{a}_{2}\right)\right]}{\Delta}>0 \\
& \frac{d \tau_{1}}{d \mathbf{R}_{1}}=-\frac{u_{S S}^{1} E_{\mathbf{R}}^{1}\left[\left(1-\mathrm{Y}_{2}\right)^{2} u_{S S}^{2} E_{\mathrm{t}}^{2}+\mathrm{a}_{2}\left(\delta+\mathrm{a}_{2}\right)\right]}{\Delta}<0 \\
& \frac{d C_{1}}{d \mathbf{R}_{1}}=\frac{E_{\mathbf{R}}^{1}\left\{a_{1}\left(\delta+a_{1}\right)\left[\left(1-x_{2}\right)^{2} u_{S S}^{2} E_{\tau}^{2}+a_{2}\left(\delta+a_{2}\right)\right]+a_{2}\left(\delta+a_{2}\right) v_{2}^{2} u_{S S}^{1} E_{\tau}^{2}\right\}}{\Delta}>0 \\
& \frac{d E_{2}}{d \mathbf{R}_{1}}=-\frac{E_{\mathbf{R}_{2}}^{1} \mathrm{a}_{2}\left(\delta+\mathrm{a}_{2}\right) \mathrm{Y}_{2} u_{S S}^{1} E_{\tau}^{2}}{\Delta}>0 \\
& \frac{d S_{2}}{d R_{1}}=-\frac{E_{R^{1}}^{1}\left(\delta+a_{2}\right) Y_{2}\left(1-\chi_{2}\right) u_{S S}^{1} E_{\tau}^{2}}{\Delta}>0 \\
& \frac{d p_{2}}{d R_{1}}=-\frac{E_{R}^{1} Y_{2}\left(1-Y_{2}\right) u_{S S}^{1} u_{S S}^{2} E_{\tau}^{2}}{\Delta}<0
\end{aligned}
$$




$$
\begin{gathered}
\frac{d \tau_{2}}{d \mathbf{R}_{1}}=-\frac{E_{\mathbf{R}_{2}}^{1} \mathrm{a}_{2}\left(\delta+\mathrm{a}_{2}\right) \mathrm{y}_{2} u_{S S}^{1}}{\Delta}<0 \\
\frac{d C_{2}}{d \mathbf{R}_{1}}=-\frac{C_{E}^{2} E_{\mathbf{R}}^{1} a_{2}\left(\delta+a_{2}\right) \mathrm{Y}_{2} u_{S S}^{1} E_{\tau}^{2}}{\Delta}>0
\end{gathered}
$$

5. Die Änderungen für ein erhöhtes $\mathbf{R}_{2}$ :

$$
\begin{aligned}
& \frac{d E_{1}}{d \mathbf{R}_{2}}=-\frac{E_{\mathbf{R}}^{2} a_{2}\left(\delta+a_{2}\right) Y_{2} u_{S S}^{1} E_{\imath}^{1}}{\Delta}>0 \\
& \frac{d S_{1}}{d \mathbf{R}_{2}}=\frac{E_{\mathrm{R}}^{2}\left(\delta+\mathrm{a}_{1}\right) \mathrm{a}_{2}\left(\delta+\mathrm{a}_{2}\right) \mathrm{Y}_{2}}{\Delta}<0 \\
& \frac{d p_{1}}{d \mathrm{R}_{2}}=\frac{E_{\mathrm{R}}^{2} u_{S S}^{1} \mathrm{a}_{2}\left(\delta+\mathrm{a}_{2}\right) \mathrm{Y}_{2}}{\Delta}>0 \\
& \frac{d \mathrm{c}_{1}}{d \mathbf{R}_{2}}=-\frac{E_{\mathbf{R}^{u}}^{2} S_{S S_{2}}^{l}\left(\delta+\mathrm{a}_{2}\right) \mathrm{Y}_{2}}{\Delta}<0 \\
& \frac{d C_{1}}{d \mathbf{R}_{2}}=-\frac{C_{E}^{1} E_{\mathrm{R}_{2}}^{2} \mathrm{a}_{2}\left(\delta+\mathrm{a}_{2}\right) \mathrm{Y}_{2} u_{S S}^{1} E_{\mathrm{r}}^{1}}{\Delta}>0
\end{aligned}
$$

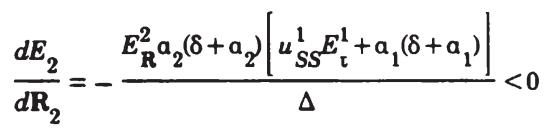

$$
\begin{aligned}
& \frac{d S_{2}}{d \mathbf{R}_{2}}=-\frac{E_{R}^{2}\left(1-Y_{2}\right)\left(\delta+a_{2}\right)\left[u_{S S}^{1} E_{\tau}^{1}+a_{1}\left(\delta+a_{1}\right)\right]}{\Delta}<0
\end{aligned}
$$




$$
\begin{aligned}
& \frac{d p_{2}}{d \mathbf{R}_{2}}=-\frac{E_{\mathbf{R}}^{2}\left(1-\mathrm{Y}_{2}\right) u_{S S}^{2}\left[u_{S S}^{1} E_{\mathrm{\tau}}^{1}+\mathrm{a}_{1}\left(\delta+\mathrm{a}_{1}\right)\right]}{\Delta}>0 \\
& \frac{d \tau_{2}}{d \mathbf{R}_{2}}=-\frac{E_{\mathbf{R}}^{2}\left\{\left(1-\mathrm{Y}_{2}\right)^{2} u_{S S}^{2}\left[u_{S S}^{1} E_{\mathrm{\tau}}^{1}+\mathrm{a}_{1}\left(\delta+\mathrm{a}_{1}\right)\right]+\mathrm{Y}_{2}^{2} \mathrm{a}_{2}\left(\delta+\mathrm{a}_{2}\right) u_{S S}^{1}\right\}}{\Delta}<0 \\
& \frac{d C_{2}}{d \mathbf{R}_{2}}=-\frac{E_{\mathbf{R}_{2}}^{2} a_{2}\left(\delta+a_{2}\right)\left[u_{S S^{1}}^{1} E_{\mathrm{c}}^{1}+a_{1}\left(\delta+a_{1}\right)\right]}{\Delta}+\mathbf{q}>0
\end{aligned}
$$

6. Die Änderungen für ein erhöhtes $\gamma_{2}$ :

Auch hier müssen zur Analyse einer Änderung des Diffusionskoeffizienten die Regionen näher spezifiziert werden, da das Verhältnis der Schattenpreise in beiden Regionen zueinander die Änderung der Steady-State-Werte bestimmt.

Es wird wieder davon ausgegangen, daß die Präferenzen - und damit die Nutzenfunktionen - und die Produktionstechnologien in beiden Regionen identisch sind. Weiterhin wird unterstellt, daß auch die Ökosysteme in beiden Regionen bezüglich des Assimilationskoeffizienten identisch sind, so daß sich die Regionen nur durch die Diffusion unterscheiden. An dem Ergebnis, das in Anhang 4.II abgeleitet wird, ändert sich nichts, d. h.es gilt

$$
p_{1}<p_{2}
$$

und damit

$$
p_{2}-p_{1}>0
$$

Für diesen Fall ergeben sich die folgenden Änderungsrichtungen [in Klammern die Änderungsrichtungen für $p_{1}>p_{2}$ ]: 


$$
\begin{aligned}
& \frac{d E_{1}}{d Y_{2}}=-\frac{u_{S S}^{1} E_{\mathrm{\tau}}^{1}\left\{\left[\left(1-Y_{2}\right) u_{S S}^{2} E_{\mathrm{t}}^{2}+a_{2}\left(\delta+a_{2}\right) \mid E_{2}+Y_{2} E_{\mathrm{t}}^{2} a_{2}\left(\delta+a_{2}\right)\left(p_{2}-p_{1}\right)\right\}\right.}{\Delta} \geq_{<0} \quad(<0) \\
& \frac{d S_{1}}{d Y_{2}}=\frac{\left(\delta+a_{1}\right)\left\{\left|\left(1-Y_{2}\right) u_{S S}^{2} E_{\mathrm{r}}^{2}+a_{2}\left(\delta+a_{2}\right)\right| E_{2}+Y_{2} E_{\tau}^{2} a_{2}\left(\delta+a_{2}\right)\left(p_{2}-p_{1}\right)\right\}}{\Delta} \geq_{<} \quad(>0)
\end{aligned}
$$$$
\frac{d p_{1}}{d \mathrm{Y}_{2}}=\frac{u_{S S}^{1}\left\{\left[\left(1-\mathrm{Y}_{2}\right) u_{S S}^{2} E_{\mathrm{\tau}}^{2}+\mathrm{a}_{2}\left(\delta+\mathrm{a}_{2}\right) \mid E_{2}+\mathrm{Y}_{2} E_{\mathrm{\tau}}^{2} \mathrm{a}_{2}\left(\delta+\mathrm{a}_{2}\right)\left(p_{2}-p_{1}\right)\right\}\right.}{\Delta} \geq_{0} \quad(<0)
$$$$
\frac{d \mathrm{t}_{1}}{d \mathrm{Y}_{2}}=-\frac{u_{S S}^{1}\left\{\left[\left(1-\mathrm{Y}_{2}\right) u_{S S}^{2} E_{\mathrm{t}}^{2}+\mathrm{a}_{2}\left(\delta+\mathrm{a}_{2}\right) \mid E_{2}+\mathrm{Y}_{2} E_{\mathrm{t}}^{2} \mathrm{a}_{2}\left(\delta+\mathrm{a}_{2}\right)\left(p_{2}-p_{1}\right)\right\}\right.}{\Delta} \geq_{<} \quad(>0)
$$

$$
\frac{d C_{1}}{d Y_{2}}=-\frac{C_{E}^{1} u_{S S}^{1} E_{\tau}^{1}\left\{\left[\left(1-Y_{2}\right) u_{S S}^{2} E_{\tau}^{2}+a_{2}\left(\delta+a_{2}\right) \mid E_{2}+Y_{2} E_{\tau}^{2} a_{2}\left(\delta+a_{2}\right)\left(p_{2}-p_{1}\right)\right\}\right.}{\Delta} \geq_{0} \quad(<0)
$$

$$
\frac{d E_{2}}{d \Upsilon_{2}}=\frac{E_{\tau}^{2}\left\{\left|u_{S S}^{1} E_{\tau}^{1}+a_{1}\left(\delta+a_{1}\right)\right|\left[\left(1-Y_{2}\right) u_{S S}^{2} E_{2}+a_{2}\left(\delta+a_{2}\right)\left(p_{2}-p_{1}\right) \mid-Y_{2} E_{2} a_{2}\left(\delta+a_{2}\right) u_{S S}^{1}\right\}\right.}{\Delta} \underset{<0}{\geq_{0}} \quad\left(\geq_{<} 0\right)
$$

$$
\frac{d S_{2}}{d Y_{2}}=-\frac{\left(\delta+a_{2}\right)\left\{\left[u_{S S}^{1} E_{\tau}^{1}+a_{1}\left(\delta+a_{1}\right)\right]\left[E_{2}-\left(1-Y_{2}\right) E_{\tau}^{2}\left(p_{2}-p_{1}\right)\right]+Y_{2} E_{\tau}^{2} E_{2} u_{S S}^{1}\right\}}{\Delta}<0 \quad(\geq<0)
$$

$$
\frac{d p_{2}}{d Y_{2}}=-\frac{u_{S S}^{2}\left\{\left[u_{S S}^{1} E_{\tau}^{1}+a_{1}\left(\delta+a_{1}\right)\right]\left[E_{2}-\left(1-Y_{2}\right) E_{\tau}^{2}\left(p_{2}-p_{1}\right)\right]+Y_{2} E_{\tau}^{2} E_{2} u_{S S}^{1}\right\}}{\Delta}>0 \quad\left(\geq_{<} 0\right)
$$




$$
\frac{d \tau_{2}}{d Y_{2}}=\frac{\left[u_{S S}^{1} E_{1}^{1}+a_{1}\left(\delta+a_{1}\right)\right]\left[\left(1-Y_{2}\right) u_{S S}^{2} E_{2}+a_{2}\left(\delta+a_{2}\right)\left(p_{2}-p_{1}\right)\right]-Y_{2} E_{2} a_{2}\left(\delta+a_{2}\right) u_{S S}^{1}}{\Delta} \geq 0 \quad\left(\geq_{<} 0\right)
$$

$\frac{d C_{2}}{d Y_{2}}=\frac{C_{E}^{2} E_{\tau}^{2}\left\{\left[u_{S S}^{1} E_{\tau}^{1}+a_{1}\left(\delta+a_{1}\right)\right]\left[\left(1-Y_{2}\right) u_{S S}^{2} E_{2}+a_{2}\left(\delta+a_{2}\right)\left(p_{2}-p_{1}\right)\right]-Y_{2} E_{2} a_{2}\left(\delta+a_{2}\right) u_{S S}^{1}\right\}}{\Delta} \geq_{<} \quad\left(\geq_{<} 0\right)$

\section{Die Änderungen für ein erhöhtes q:}

$$
\begin{aligned}
& \frac{d E_{1}}{d \mathbf{q}}=\frac{a_{1}\left(\delta+a_{1}\right) E_{\mathbf{q}}^{1}\left[\left(1-Y_{2}\right)^{2} u_{S S}^{2} E_{\mathrm{t}}^{2}+a_{2}\left(\delta+a_{2}\right)\right]-a_{2}\left(\delta+a_{2}\right) u_{S S}^{1} Y_{2}\left(E_{\tau}^{1} E_{q}^{2}-Y_{2} E_{q}^{1} E_{\tau}^{2}\right)}{\Delta} \geq_{0} \\
& \frac{d S_{1}}{d \mathbf{q}}=\frac{\left(\delta+a_{1}\right)\left\{\left[\left(1-Y_{2}\right)^{2} u_{S S}^{2} E_{\tau}^{2}+a_{2}\left(\delta+a_{2}\right) \mid E_{\mathbf{q}}^{1}+Y_{2} E_{q}^{2} a_{2}\left(\delta+a_{2}\right)\right\}\right.}{\Delta} \geq_{0}
\end{aligned}
$$$$
\frac{d p_{1}}{d \mathbf{q}}=\frac{u_{S S}^{1}\left\{\left[\left(1-\mathrm{Y}_{2}\right)^{2} u_{S S}^{2} E_{\mathrm{t}}^{2}+\mathrm{a}_{2}\left(\delta+\mathrm{a}_{2}\right) \mid E_{\mathrm{q}}^{1}+\mathrm{Y}_{2} E_{\mathrm{q}}^{2} \mathrm{a}_{2}\left(\delta+\mathrm{a}_{2}\right)\right\}\right.}{\Delta} \geq_{<}
$$

$$
\frac{d \tau_{1}}{d \mathbf{q}}=-\frac{u_{S S}^{1}\left\{\left[\left(1-\mathrm{Y}_{2}\right)^{2} u_{S S}^{2} E_{\mathrm{t}}^{2}+\mathrm{a}_{2}\left(\delta+\mathrm{a}_{2}\right)\right] E_{\mathrm{q}}^{1}+\mathrm{Y}_{2} E_{\mathrm{q}}^{2} \mathrm{a}_{2}\left(\delta+\mathrm{a}_{2}\right)\right\}}{\Delta} \geq 0
$$$$
\frac{d C_{1}}{d \mathbf{q}}=C_{E}^{1} \frac{d E_{1}}{d \mathbf{q}}-M_{1} \geq 0
$$

$$
\begin{aligned}
& \frac{d E_{2}}{d \mathbf{q}}=\frac{a_{2}\left(\delta+a_{2}\right)\left\{\left[u_{S S}^{1} E_{\tau}^{1}+a_{1}\left(\delta+a_{1}\right)\right] E_{q}^{2}-Y_{2} E_{\tau}^{2} E_{q}^{1} u_{S S}^{1}\right\}}{\Delta} \geq 0 \\
& \frac{d S_{2}}{d q}=\frac{\left(1-Y_{2}\right)\left(\delta+a_{2}\right)\left\{\left[u_{S S}^{1} E_{\tau}^{1}+a_{1}\left(\delta+a_{1}\right)\right] E_{q}^{2}-Y_{2} E_{\tau}^{2} E_{q}^{1} u_{S S}^{1}\right\}}{\Delta}<0
\end{aligned}
$$


$\frac{d p_{2}}{d \mathbf{q}}=-\frac{\left(1-\mathrm{Y}_{2}\right) u_{S S}^{2}\left\{\left[u_{S S^{1}}^{1} E_{\tau}^{1}+\mathrm{a}_{1}\left(\delta+\mathrm{a}_{1}\right) \mid E_{\mathrm{q}}^{2}-\mathrm{Y}_{2} E_{\tau}^{2} E_{\mathrm{q}}^{1} u_{S S}^{1}\right\}\right.}{\Delta} \geq 0$

$\frac{d \tau_{2}}{d \mathbf{q}}=-\frac{\left|u_{S S}^{1} E_{\tau}^{1}+a_{1}\left(\delta+a_{1}\right)\right|\left(1-Y_{2}\right)^{2} u_{S S}^{2} E_{q}^{2}+Y_{2}\left(E_{q}^{1}+Y_{2} E_{q}^{2}\right) a_{2}\left(\delta+a_{2}\right) u_{S S}^{1}}{\Delta}<0$

$\frac{d C_{2}}{d \mathbf{q}}=C_{E}^{2} \frac{d E_{2}}{d \mathbf{q}}-M_{2} \geq 0$ 


\section{ANHANG 6.III: Standardsetzung}

1. Die Änderungen für Region 2

Für Region 2 muß folgendes Gleichungssystem betrachtet werden unter der Annahme, daß der Emissionsstandard bindend ist:

$$
\begin{aligned}
\left(1-\gamma_{2}\right) \mathbf{E}_{2}-\alpha_{2} S_{2} & =0 \\
\left(\delta+\alpha_{2}\right) p_{2}-u^{2}{ }_{S} & =0 \\
\tau_{2}+\left(1-\gamma_{2}\right) p_{2}-\lambda^{2}{ }_{2} & =0 \\
\mathbf{E}_{2}-E^{2}\left(\tau_{2}\right) & =0
\end{aligned}
$$

Man erhält

$\left[\begin{array}{cccc}-\alpha_{2} & 0 & 0 & 0 \\ -u^{2} S S & \delta+\alpha_{2} & 0 & 0 \\ 0 & 1-\gamma_{2} & 1 & -1 \\ 0 & 0 & -E^{2} \tau^{2} & 0\end{array}\right]\left[\begin{array}{l}d S_{2} \\ d p_{2} \\ d \tau_{2} \\ d \lambda_{2}{ }^{2}\end{array}\right]=\left[\begin{array}{c}0 \\ -p_{2} \\ 0 \\ 0\end{array}\right] d \delta+\left[\begin{array}{c}S_{2} \\ -p_{2} \\ 0 \\ 0\end{array}\right] d \alpha_{2}+$

$$
+\left[\begin{array}{c}
0 \\
0 \\
0 \\
E_{\mathbf{R}}^{2}
\end{array}\right] d \mathbf{R}_{2}+\left[\begin{array}{c}
\mathbf{E}_{2} \\
0 \\
p_{2} \\
0
\end{array}\right] d \gamma_{2}+\left[\begin{array}{c}
-\left(1-\gamma_{2}\right) \\
0 \\
0 \\
-1
\end{array}\right] d \mathbf{E}_{2}+\left[\begin{array}{c}
0 \\
0 \\
0 \\
E_{q}^{2}
\end{array}\right] d \mathbf{q}
$$


1. Die Änderungen für ein erhöhtes $\gamma_{2}$ :

$$
\begin{aligned}
& \frac{d S_{2}}{d \mathrm{Y}_{2}}=\frac{-\mathbf{E}_{2}}{a_{2}}<0 \\
& \frac{d p_{2}}{d \mathrm{Y}_{2}}=-\frac{u_{S S}^{2} \mathrm{E}_{2}}{a_{2}\left(\delta+a_{2}\right)}>0 \\
& \frac{d \tau_{2}}{d \mathrm{Y}_{2}}=0 \\
& \frac{d \mathrm{\lambda}_{2}^{2}}{d \mathrm{Y}_{2}}=-\frac{\left(1-\mathrm{Y}_{2}\right) u_{S S}^{2} \mathrm{E}_{2}}{\mathrm{a}_{2}\left(\delta+\mathrm{a}_{2}\right)}>0 \\
& \frac{d C_{2}}{d \mathrm{Y}_{2}}=0
\end{aligned}
$$

2. Die Änderungen für ein erhöhtes $\delta$ :

$$
\begin{aligned}
& \frac{d S_{2}}{d \delta}=0 \\
& \frac{d p_{2}}{d \delta}=\frac{-p_{2}}{\delta+\mathrm{a}_{2}}>0 \\
& \frac{d \tau_{2}}{d \delta}=0 \\
& \frac{d \mathrm{\Lambda}_{2}^{2}}{d \delta}=-\frac{\left(1-\mathrm{Y}_{2}\right) p_{2}}{\delta+\mathrm{a}_{2}}>0
\end{aligned}
$$




$$
\frac{d C_{2}}{d \delta}=0
$$

3. Die Änderungen für ein erhöhtes $\alpha_{2}$ :

$$
\begin{aligned}
& \frac{d S_{2}}{d a_{2}}=\frac{-S_{2}}{a_{2}}<0 \\
& \frac{d p_{2}}{d a_{2}}=\frac{-\left[u_{S S}^{2} S_{2}+a_{2} p_{2}\right]}{a_{2}\left(\delta+a_{2}\right)}>0 \\
& \frac{d \tau_{2}}{d a_{2}}=0 \\
& \frac{d \lambda_{2}^{2}}{d a_{2}}=-\frac{\left(1-x_{2}\right)\left|u_{S S}^{2} S_{2}+a_{2} p_{2}\right|}{a_{2}\left(\delta+a_{2}\right)}<0 \\
& \frac{d C_{2}}{d a_{2}}=0
\end{aligned}
$$

4. Die Änderungen für ein erhöhtes $\mathbf{R}_{2}$ :

$$
\begin{aligned}
& \frac{d S_{2}}{d \mathbf{R}_{2}}=0 \\
& \frac{d p_{2}}{d \mathbf{R}_{2}}=0 \\
& \frac{d \tau_{2}}{d \mathbf{R}_{2}}=\frac{-E_{\mathbf{R}}^{2}}{E_{\tau}^{2}}>0
\end{aligned}
$$




$$
\begin{aligned}
& \frac{d \mathrm{\lambda}_{2}^{2}}{d \mathbf{R}_{2}}=\frac{-E_{\mathrm{R}}^{2}}{E_{\mathrm{t}}^{2}}<0 \\
& \frac{d C_{2}}{d \mathbf{R}_{2}}=\mathrm{q}>0
\end{aligned}
$$

5. Die Änderungen für ein erhöhtes $E_{2}$ :

$$
\begin{aligned}
& \frac{d S_{2}}{d \mathbf{E}_{2}}=\frac{1-\mathrm{Y}_{2}}{\mathrm{a}_{2}}>0 \\
& \frac{d p_{2}}{d \mathbf{E}_{2}}=\frac{\left(1-\mathrm{Y}_{2}\right) u_{S S}^{2}}{\mathrm{a}_{2}\left(\delta+\mathrm{a}_{2}\right)}>0 \\
& \frac{d \tau_{2}}{d \mathbf{E}_{2}}=\frac{1}{E_{\tau}^{2}}>0 \\
& \frac{d \lambda_{2}^{2}}{d \mathbf{E}_{2}}=\frac{a_{2}\left(\delta+a_{2}\right)+\left(1-Y_{2}\right)^{2} E_{\tau}^{2} u_{S S}^{2}}{a_{2}\left(\delta+a_{2}\right) E_{\tau}^{2}}<0 \\
& \frac{d C_{2}}{d \mathbf{E}_{2}}=C_{E}^{2}>0
\end{aligned}
$$

6. Die Änderungen für ein erhöhtes q:

$$
\begin{aligned}
& \frac{d S_{2}}{d \mathbf{q}}=0 \\
& \frac{d p_{2}}{d q}=0
\end{aligned}
$$




$$
\begin{aligned}
& \frac{d \tau_{2}}{d \mathbf{q}}=-\frac{E_{\mathrm{q}}^{2}}{E_{\mathrm{\tau}}^{2}}<0 \\
& \frac{d \lambda_{2}^{2}}{d \mathbf{q}}=-\frac{E_{\mathbf{q}}^{2}}{E_{\mathrm{\tau}}^{2}}<0 \\
& \frac{d C_{2}}{d \mathbf{q}}=-M_{2} \geq 0 \Leftrightarrow \mathrm{R}_{2} \geq R_{2}
\end{aligned}
$$

\section{Die Änderungen für Region 1}

Für Region 1 erhalten wir ein analoges Gleichungssystem zu demjenigen autonomer Regionalbehörden [vgl. Anhang 6.I]:

$$
\begin{aligned}
E_{1}-\alpha_{1} S_{1} & =-\gamma_{2} \mathbf{E}_{2} \\
\left(\delta+\alpha_{1}\right) p_{1}-u_{S}^{1} & =0 \\
\tau_{1}+p_{1} & =0 \\
E_{1}-E^{1}\left(\tau_{1}\right) & =0
\end{aligned}
$$

Totale Differentation ergibt

$\left[\begin{array}{cccc}1 & -\alpha_{1} & 0 & 0 \\ 0 & -u_{S S}^{1} & \delta+\alpha_{1} & 0 \\ 0 & 0 & 1 & 1 \\ 1 & 0 & 0 & -E^{1} \tau\end{array}\right]\left[\begin{array}{l}d E_{1} \\ d S_{1} \\ d p_{1} \\ d \tau_{1}\end{array}\right]=\left[\begin{array}{c}E_{2} \\ 0 \\ 0 \\ 0\end{array}\right] d \gamma_{2}+\left[\begin{array}{l}0 \\ -p_{1} \\ 0 \\ 0\end{array}\right] d \delta+$


$+\left[\begin{array}{c}S_{1} \\ -p_{1} \\ 0 \\ 0\end{array}\right] d \alpha_{1}+\left[\begin{array}{c}0 \\ 0 \\ 0 \\ E^{1}\end{array}\right] d \mathbf{R}_{1}+\left[\begin{array}{c}-\gamma_{2} \\ 0 \\ 0 \\ 0\end{array}\right] d \mathbf{E}_{2}+\left[\begin{array}{c}0 \\ 0 \\ 0 \\ E^{1}\end{array}\right]$

Die Systemdeterminante ist

$$
\Delta_{1}=u_{S S^{1}}^{E^{1}}+\alpha_{1}\left(\delta+\alpha_{1}\right)>0
$$

2.1 Die Änderungen für ein größeres $\gamma_{2}$ :

$$
\begin{aligned}
& \frac{d E_{1}}{d \mathrm{Y}_{2}}=-\frac{E_{\mathrm{\tau}}^{1} u_{S S}^{1} \mathbf{E}_{2}}{\Delta_{1}}<0 \\
& \frac{d S_{1}}{d \mathrm{Y}_{2}}=\frac{\left(\delta+a_{1}\right) \mathbf{E}_{2}}{\Delta_{1}}>0 \\
& \frac{d p_{1}}{d \mathrm{Y}_{2}}=\frac{u_{S S}^{1} \mathbf{E}_{2}}{\Delta_{1}}<0 \\
& \frac{d \tau_{1}}{d \mathrm{Y}_{2}}=\frac{-u_{S S}^{1} \mathbf{E}_{2}}{\Delta_{1}}>0 \\
& \frac{d C_{1}}{d \mathrm{Y}_{2}}=-\frac{C_{E}^{1} E_{\tau}^{1} u_{S S}^{1} \mathbf{E}_{2}}{\Delta_{1}}<0
\end{aligned}
$$

2.2 Die Änderungen für ein erhöhtes $\delta$ :

$$
\frac{d E_{1}}{d \delta}=\frac{E_{\tau}^{1} a_{1} p_{1}}{\Delta_{1}}>0
$$




$$
\begin{aligned}
& \frac{d S_{1}}{d \delta}=\frac{E_{\tau}^{1} p_{1}}{\Delta_{1}}>0 \\
& \frac{d p_{1}}{d \delta}=\frac{-a_{1} p_{1}}{\Delta_{1}}>0 \\
& \frac{d \tau_{1}}{d \delta}=\frac{a_{1} p_{1}}{\Delta_{1}}<0 \\
& \frac{d C_{1}}{d \delta}=\frac{C_{E}^{1} E_{\tau}^{1} a_{1} p_{1}}{\Delta_{1}}>0
\end{aligned}
$$

2.3 Die Änderungen für ein erhöhtes $\alpha_{1}$ und $\mathbf{R}_{1}$ :

Hier ändert sich gegenüber dem autonomen Fall nichts, so daß auf eine Ableitung verzichtet wird [vgl. Anhang 6.I, 2.3 und 2.4].

2.4 Die Änderungen in Region 1 bei erhöhtem $\alpha_{2}$ und $R_{2}$ :

Da der Emissionsstandard bindend ist, besteht keine Abhängigkeit von $\alpha_{2}$ und $\mathbf{R}_{2}$ für Region 1. Es gilt

$$
\frac{d E_{1}}{d a_{2}}=\frac{d S_{1}}{d a_{2}}=\frac{d p_{1}}{d a_{2}}=\frac{d \tau_{1}}{d a_{2}}=\frac{d E_{1}}{d \mathbf{R}_{2}}=\frac{d S_{1}}{d \mathbf{R}_{2}}=\frac{d p_{1}}{d \mathbf{R}_{2}}=\frac{d \tau_{1}}{d \mathbf{R}_{2}}=0
$$

2.5 Die Änderungen in Region 1 bei erhöhtem q:

Hier ändert sich gegenüber dem Fall ohne Diffusion des Kapitels 5 hichts, so daß auf die dortige Ableitung verwiesen werden kann. 
Helga Gebauer - 978-3-631-75599-0

Downloaded from PubFactory at 01/11/2019 03:14:13AM

via free access 


\section{Kapitel 7: Schlußbetrachtung}

Das Hauptanliegen dieser Arbeit ist, die intertemporalen Zusammenhänge von regionalisierten Umweltpolitiken zu untersuchen. Dabei werden vor allem solche Phänomene berücksichtigt, die sich dadurch ergeben, daß politische bzw. ökonomische Gebiete nicht identisch sind mit ökologischen Teilräumen (Luftbecken, Wassersysteme).

Innerhalb dieser Schlußbetrachtung werden nicht noch einmal die Ergebnisse referiert, sondern es wird eine zusammenfassende Wertung der Auswirkungen der unterschiedlichen institutionellen Gegebenheiten diskutiert, eine Kategorisierung der Parameter gegeben, die das Gleichgewicht mitbestimmen sowie ein Ausblick auf Anknüpfungspunkte für weitere Forschungsarbeiten gegeben.

Durch die notwendigen vereinfachenden Modellannahmen sind keine unmittelbar verwertbaren Resultate für die aktuelle Wirtschaftspolitik zu erzielen, jedoch werden die ökonomischen Zusammenhänge deutlich, die sich durch die Schadstoffdiffusion und die Handelsbeziehungen auf die Umweltpolitik in einem intertemporalen Kontext ergeben.

Eine Rangfolge der "Regierungsformen" hinsichtlich der Effizienz kann, unabhängig davon, ob es sich um autarke Regionen handelt oder die Regionen an einem Weltmarkt beteiligt sind, folgendermaßen dargestellt werden:

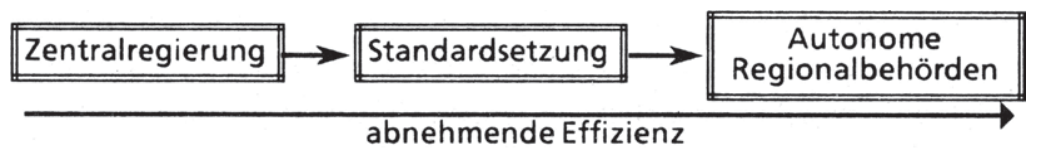

Schaubild 7.1: Die Effizienzeigenschaften der institutionellen Ausgestaltungen

Wird die Umweltpolitik und damit die Optimierung von einer Zentralregierung vorgenommen, ist das sich ergebende Steady-State Pareto-optimal. Zwar wird das Ökosystem einer emissionsimportierenden Region durch regionenfremde Emissionen belastet, jedoch wird der gesamte Schaden, der durch die Emissionstätigkeit entsteht, bei der Optimierung be- 
rücksichtigt. Nicht nur der Immissionsbestand in der emissionsexportierenden Region wird von der zentralen Umweltbehörde bei den Produktionsentscheidungen für diese Region berücksichtigt, sondern auch die sozialen Kosten, die in der anderen Region entstehen, werden mit ins Kalkül gezogen.

Durch diese Form der Optimierung werden die spill-over Effekte internalisiert. Eine solche Lösung ist deshalb nur innerhalb eines Staates zu erwarten, der zentral geführt wird.

Interpretiert man dieses Vorgehen als kooperatives Spiel zwischen zwei Staaten, d. h. zwei Staaten einigen sich auf eine gemeinsame Wohlfahrtsoptimierung und bestimmen das Gewicht mit dem ihre jeweilige Wohlfahrt in das Optimierungsproblem eingeht, hat der emissionsimportierende Staat eine "schwache" Verhandlungsposition. Eine solche Lösung verlangt von dem emissionsexportierenden Staat eine extrem altruistische Haltung, so daß es wenig wahrscheinlich erscheint, daß sich solch eine Verhandlungslösung einstellt.

Der diametral entgegengesetzte Ansatz vollkommen autonomer Regionalbehörden ist sicherlich die realistischere Variante, wie auch schon in Kapitel 2 im Zusammenhang mit der Reaktion auf die Forderung der skandinavischen Länder nach Begrenzung der weiträumigen Luftverschmutzung im restlichen Europa angedeutet wird. Ein aktuelles Beispiel ist die Versalzung des Rheins durch die Kalibergwerke im Elsaß. Die Niederlande, die ihr Trinkwasser aus dem Rhein gewinnen, sind gezwungen, die Auswirkungen der interregionalen spill-overs auszugleichen. Hier muß die emissionsimportierende Region die sozialen Kosten des Emissionsimports allein tragen. Damit hat sie Restriktionen bei der Ausgestaltung ihrer Umweltpolitik zu beachten, die außerhalb ihres Einflußbereiches liegen. Die emissionsimportierende Region muß ihre Umweltpolitik nach der in der emissionsexportierenden Region betriebenen Umweltpolitik ausrichten.

Das dritte institutionelle Arrangement autonomer Regionalbehörden mit einem vorgegebenen Emissionsstandard für die emissionsexportierende Region kann hinsichtlich der Effizienzeigenschaften folgendermaßen charakterisiert werden: Hat die Zentralbehörde ausreichend großen Einfluß, dann wird ein Emissionsstandard gesetzt, der die gesamten sozialen Ko- 
sten, die durch den Emissionsimport in Region 1 entstehen, bei der Umweltpolitik der autonomen Region 2 berücksichtigt. Dann ist das sich ergebende Gleichgewicht ebenfalls Pareto-optimal. Durch eine Standardsetzung für die emissionsexportierende Region, die im politischen Prozeß leichter durchzusetzen sein dürfte als eine gemeinsame Optimierung durch eine Zentralregierung, können zumindest teilweise die sozialen Kosten dem Verschmutzer zugeordnet werden, wenn auch nicht zu erwarten ist, daß die gesamten Schäden, wie bei einer Zentralregierung, berücksichtigt werden.

Die indirekten und die direkten interregionalen Verflechtungen zeigen unterschiedliche Auswirkungen bei Variation der das Gleichgewicht mitbestimmenden Parameter. Dazu können vier Kategorien gebildet werden:

- Parameter, deren Einfluß auf die Höhe der Gleichgewichtsvariablen unabhängig ist davon, ob die Ökonomie autark ist oder nicht, bzw. die Regionen über Diffusionsbeziehungen verbunden sind oder nicht.

- Parameter, deren Einfluß hauptsächlich über die Diffusionsbeziehungen wirkt;

- Parameter, deren Einfluß hauptsächlich über Handelsbeziehungen wirkt;

- Parameter, die sowohl von den direkten als auch den indirekten Auswirkungen interregionaler Verflechtung abhängen.

In die erste Kategorie der unabhängigen Parameter fällt die Assimilationskapazität des Ökosystems. Die Auswirkungen der Größe der Assimilationskoeffizienten einer Region hat der Richtung nach jeweils die gleichen Auswirkungen für diese Region in allen betrachteten Fällen: Die Emissionen und der Konsum steigen, während der bewertete Schaden und die Steuer sinkt. Die Bedingung für die Änderung der Immissionen bleibt grundsätzlich unabhängig von der Handelsposition der Region und von der Diffusion der Schadstoffe. Daß durch die Diffusionsbeziehungen Rückwirkungen auf die andere Region entstehen, ist für diese Kategorisierung nicht von entscheidender Bedeutung, da für die jeweilige Region die Beziehungen erhalten bleiben.

In die zweite Kategorie gehören die Auswirkungen der Höhe der sozialen Diskontrate, bei der die Änderungsrichtungen im autarken Fall in 
Kapitel 4 äquivalent sind zu denen im Kapitel 6 betrachteten Fall mit Handelsbeziehungen. Auch die Auswirkungen der Höhe des Diffusionskoeffizienten gehören in diese Kategorie.

In die dritte Kategorie gehören die Auswirkungen der Größe des Faktorbestandes auf das Steady-State. Durch die Handelsbeziehungen werden die Vorzeichen der Änderungen der Steady-State-Werte der Produktion, der Emissionen und der Steuern gerade umgekehrt, während der Konsum für einen höheren Faktorbestand in beiden Fällen steigt. Auch die Auswirkungen der Höhe des Faktorbestandes in der emissionsexportierenden Region 2 auf die emissionsimportierende Region 1 sind bei Handelsbeziehungen genau umgekehrt zu dem autarken Fall.

In die vierte Kategorie schließlich wird die Höhe des Relativpreises eingeordnet, da die Auswirkungen auf die emissionsimportierende Region 1 entscheidend mit von den Diffusionsbeziehungen abhängen, während die Auswirkungen auf die emissionsexportierende Region 2 äquivalent sind zu denen ohne Diffusionsbeziehungen.

Diese Kategorisierung der Parameter kann unabhängig davon vorgenommen werden, welche institutionelle Ausgestaltung der Ökonomie vorliegt. Dabei ist jedoch zu beachten, daß die Auswirkungen der Höhe des Relativpreises auch abhängig sind von der Regierungsform.

Bei den Diffusionsbeziehungen haben wir uns auf die "Unterlieger-Oberlieger-Problematik" sowie auf den Zwei-Regionen-Fall beschränkt. Natürliche Erweiterungen für diese Fragestellungen sind die gegenseitige Verschmutzung bzw. die Verschmutzung eines Umweltmediums, das Allmende-Gut für mehrere Regionen ist. Darüberhinaus sind die Handelsbeziehungen der Regionen durch eine einfache Struktur geprägt. Einzubeziehen wäre etwa Faktormobilität im Sinne von Konsumentenwanderung. Die Rückwirkungen der Umweltpolitik auf die Höhe des Relativpreises können durch Aufgabe der Annahmen des kleinen Landes und des daraus resultierenden konstanten Relativpreises mit in die Analyse einbezogen werden. 


\section{Literaturverzeichnis}

d'Arge, R. C. und K. C. Kogiku (1973), Economic Growth and the Environment. Review of Economic Studies 40,61-77.

Arnold, V. (1984), Umweltschutz als international öffentliches Gut:

Komparative Kostenvorteile und Verhandlungsgewinne. Zeitschrift

für Wirtschafts- und Sozialwissenschaften, 111-129.

Arrow, K. J. (1963), Social Choice and Individual Values. New York.

Arrow, K. J. (1968), Applications of Control Theory to Economic Growth.

In: G. B. Dantzig und A.F. Veinott Jr. (Hrsg.), Mathematics of the Decision Sciences, Part 2, 85-119.

Arrow, K. J. und M. Kurz (1970), Optimal Growth with Irreversible Investment in a Ramsey Model. Econometrica 38, 331-344.

Asako, K. (1979), Environmental Pollution in an Open Economy. The Economic Record 55, 359-367.

Athans, M. und P. L. Falb (1966), Optimal Control. New York u. a..

Ayres, R. U. (1978), Resources, Environment, and Economics. Applications of the Materials/Energy Balance Principle. New York u. a..

Bailey, M. J. (1982), Risks, Costs, and Benefits of Fluorocarbon Regulation. Papers and Proceedings of the Ninty-Fourth Annual Meeting of the American Economic Association 72, 247-250.

Basar, T. und G. J. Olsder (1982), Dynamic Noncooperative Game Theory. London u. a..

Basta, D. J. und B. T. Bower (Hrsg.) (1982), Analyzing Natural Systems. Analysis for Regional Residuals-Environmental Quality Management, Washington, D. C..

Basta, D. J. und D. H. Moreau (1982), Introduction to Analyzing Natural Systems. In: D. J. Basta und B. T. Bower (Hrsg.), 23-120. 
Baumol, W. J. und W. E. Oates (1975), The Theory of Environmental Policy: Externalities, Public Outlaws, and the Quality of Life. Englewood Cliffs, N. J..

Bender, D. (1976), Makroökonomik des Umweltschutzes. Göttingen.

Bernstein, J.E. (1977), A Noncooperative Model of Public Investment and International Externalities. International Economic Review 18, 393 406.

Bewley, T. F. (1981), A Critique of Tiebout's Theory of Local Public Expenditures. Econometrica 49, 713-740.

Blümel, W., R. Pethig und O. von dem Hagen (1984), Theory of Public Goods: A Survey of Recent Issues. Manuskript.

Bonus, $H$. (1981), Emissionsrechte als Mittel der Privatisierung öffentlicher Ressourcen aus der Umwelt. In: L. Wegehenkel (Hrsg.), Marktwirtschaft und Umwelt, Tübingen.

Bower, B. T. und D. J. Basta (1982), Introduction: Analysis for ResidualsEnvironmental Quality Management (REQM). In: D. J. Basta und B. T. Bower (Hrsg.), Chapter One, 1-21.

Breton, A. (1970), Public Goods and the Stability of Federalism. Kyklos 23, 882-902.

Bryson, A.E. und Y.C.Ho(1975), Applied Optimal Control. Revised Printing, Washington.

Buchanan, J. M. (1965), An Economic Theory of Clubs. Economica 32, 114.

Bundesministerium des Innern BMI (1982), Umwelt. Informationen des Bundesministers des Innern zur Umweltplanung und zum Umweltschutz.

Bundestagsdrucksache 9/1955. 
Calder, K. L. (1949), Eddy Diffusion and Evapuration in Flow Over Aerodynamically Smooth and Rough Surfaces. Quarterly Journal of Mechanical and Applied Mathematics 2, 153-176.

Calder, K. L. (1965), On the Equation of Atmospheric Diffusion. Quarterly Journal of the Royal Meteorological Society 91, 514-517.

Calder, K. L. (1971), A Climatological Model for Multiple Source Urban Air Pollution. NATO/CCMS, Air Pollution 5.

Case, J.H. (1967), Equilibrium Points of N-Person Differential Games. University of Michigan Discussion Paper TR No. 1967-1.

Christensen, O. und L. P. Prahm (1976), A Pseudospectral Model for Dispersion of Atmospheric Pollutants. Journal of Applied Meteorology 15, 1284-1294.

Clark, W. C., D. D. Jones und C. S. Holling (1979), Lessons for Ecological Policy Design: A Case Study of Ecosystem Management. Ecological Modelling 7, 1-53.

Comolli, P. M. (1975), Dynamic General-Equilibrium Models in Environmental Economics. Ph. D. Dissertation, Purdue University, West Lafayette, Ind..

Conrad, K. (1976), Einbeziehung von Umweltbelastungen und Entsorgungsleistungen in preisabhängige Input-Output-Koeffizienten. In: $P$. J. Jansen, O. Moeschlin und O. Rentz (Hrsg.), Quantitative Modelle für ökonomisch-ökologische Analysen. Meisenheim am Glan, 127-153.

Converse, A. O. (1974), Environmental Controls and Economic Growth. Journal of Economic Theory 7, 411-417.

Courant, $R$. (1972), Vorlesungen über Differential- und Integralrechnung 2.

Crank, J. (1975), The Mathematics of Diffusion. Second Edition, Oxford.

Csanady, G. T. (1973), Turbulent Diffusion in the Environment. Boston. 
Dales, J. H. (1968), Pollution, Property and Prices. Toronto.

Dasgupta, P. (1969), On the Concept of Optimum Population. Review of Economic Studies 36, 295-318.

Dobbins, R.A. (1979), Atmospheric Motion and Air Pollution. New York u. a..

van Dop, $H$. (1983), The Residence and Transport of Pollutants in the Atmosphere - A Meteorological Problem. In: H.Ott und H. Stangl (Hrsg.) (1983), Symposium Saure Niederschläge. Eine Herausforderung für Europa. Proceedings - Preliminary Edition - , Karlsruhe, 4857.

Dowing, T.E. und R.W. Kates (1982), The International Response to the Threat of Chlorofluorocarbons to Atmospheric Ozone. Papers and Proceedings of the Ninty-Fourth Annual Meeting of the American Economic Association 72, 267-272.

Dudenhöffer, F . (1979), Umweltpolitik in einem föderativen System. Beiträge zur angewandten Wirtschaftsforschung, Universität Mannheim, Discussion Paper 134/79.

Dudenhöffer, $F$. (1983), Mehrheitswahlentscheidungen über Umweltnutzungen. Frankfurt/ Main u. a..

Dudenhöffer, $F$. (1983), Regulation of Intensities and Productivities: Concepts in Environmental Policy. Zeitschrift für die gesamte Staatswissenschaft (in Vorbereitung).

Dudenhöffer, F. und H. Gebauer (1982), Die Allokation öffentlicher Güter bei Konsumentenmobilität. Eine Anmerkung zum Tiebout-Theorem. In: H. Siebert (Hrsg.), Umweltallokation im Raum, Frankfurt/Main, Bern, 93-144.

Ellenberg, $H$. (1973), Ziele und Stand der Ökosystemforschung. In: $H$. Ellenberg (Hrsg.), Ökosystemforschung. Berlin. 
Ercman, S. (1977), European Environmental Law. Legal and Economic Appraisal. Bern.

Evans, A. (1978), Neighborhood Externalities, Economic Clubs, and the Environment. In: Public Economics and the Quality of Life, hrsg. von L. Wings und A. Evans, Baltimore.

Evans, A. (1980), Some Extensions of the Theory of Regional Growth and Environmental Policy. In: Siebert, I. Walter und K. Zimmermann (Hrsg.), 138-147.

Faber, M. (1984), A Biophysical Approach to the Economy. Entropy, Environment and Resources. Heidelberger Diskussionsschrift.

Faber, M., H. Niemes und G. Stephan (1983), Entropie, Umweltschutz und Rohstoffverbrauch. Eine naturwissenschaftlich ökonomische Untersuchung. Berlin u. a..

Fisher, A. C., J. V. Krutilla und C. J. Cicchetti (1972), The Economics of Environmental Preservation: A Theoretical and Empirical Analysis. American Economic Review 62, 605-619.

Fisher, A.C. und F. M. Peterson (1976), The Environment in Economics: A Survey. Journal of Economic Literature 14, 1-33.

Forster, B. A. (1973), Optimal Consumption Planning in a Polluted Environment. Economic Record 49, 534-545.

Forster, B. A. (1977a), On a One State Variable Optimal Control Problem, Consumption-Pollution Trade-Offs. In: J.D. Pitchford und S. J. Turnovsky (Hrsg.), Applications of Control Theory to Economic Analysis, 35-56.

Forster, B. A. (1977b), Pollution Control in a Two-Sector Dynamic General Equilibrium Model. Journal of Environmental Economics and Management 4, 305-312. 
Forster, B. A. (1978), An Approach to the Optimal Control of Pollution in Boundary Waters. University of Guelph, Economics Discussion Paper 78-1.

Forster, B. A. (1982), Economic Impact of Acid Precipation: A Canadian Perspective. University of Guelph, Economics Discussion Paper 82-2.

Forster, B. A. und J. Logan (1973), The Welfare Economics of Water Resource Allocation over Time: Comment. Applied Economics 5.

Førsund, F. R. (1980), Dynamic Aspects of Regional Environmental Policy. In: H. Siebert. I. Walter und K. Zimmermann (Hrsg.), 122-138.

Frey, R. L. (1977), Zwischen Föderalismus und Zentralismus. Ein volkswirtschaftliches Konzept des schweizerischen Bundesstaates. Bern, Frankfurt/Main.

Frey, R. L. (1980), Interregional Welfare Comparisons and Environmental Policy. In: H. Siebert, I. Walter und K. Zimmermann (Hrsg.), 97-108.

Fronza, G., A. Spirito und A. Tonielli (1979), The Kalman Predictor. In: International Institute for Applied Systems Analysis (Hrsg.), Real-Time Forecasting of Air Pollution Episodes in the Venetian Region. Laxenburg, Österreich, 21-42.

Gale, D. und H. Nikaido (1965), The Jacobian Matrix and Global Univalence of Mappings. Mathematische Annalen 159, 81-93.

Gandolfo, G. (1980), Economic Dynamics: Methods and Models. Amsterdam u. a., 2. verbesserte Auflage.

Gebauer, $H$. (1982), Zur intertemporalen regionalen Umweltallokation. In: H. Siebert (Hrsg.), Umweltallokation im Raum, Frankfurt/Main, Bern, 191-228. 
Gebauer, H. (1984), Politik des hohen Schornsteins und Schadstoffakkumulation. Eine intertemporale Zwei-Regionen-Analyse. In: H. Siebert (Hrsg.), Intertemporale Allokation, Frankfurt/Main u. a. (in Vorbereitung).

Georgescu-Roegen, N. (1971), The Entropy Law and the Economic Process. Cambridge, Mass., 3. Auflsge.

Gladwin, T.N., J. L. Ugelow und I. Walter (1982), Approaches to International Negotiations on the Chlorofluorcarbon Problem. In: H. Siebert (Hrsg.), Global Environmental Resources, Frankfurt/Main, Bern, 1-55.

Gottwald, D. (1981), Die dynamische Theorie der Allokation erschöpfbarer Ressourcen. Göttingen.

Graedel, T.E., L. A. Farrow und T.E. Weber (1976), Kinetic Studies of the Photochemistry of the Urban Troposphere. Atmospheric Environment 10,1095-1116.

Gronych, $R$. (1980), Allokationseffekte und Außenhandelswirkungen der Umweltpolitik. Eine komparativ-statische Zwei-Sektor-Analyse. Tübingen.

Hadley, G. und M. C. Kemp (1971), Variational Methods in Economics. Amsterdam.

von dem Hagen, O. (1983), Aspects of the Theory of Local Public Goods. Manuskript.

Hamlen Jr., W. A. (1978), The Optimality and Feasibility of Uniform Air Pollution Controls. Journal of Environmental Economics and Management 5, 301-312.

Hartkopf, G. und E. Bohne (1983a), Die Ausgangssituation unserer Umweltpolitik. Aus Politik und Zeitgeschichte, Beilage zur Wochenzeitung Das Parlament B 42, 3-12. 
Hartkopf, G. und E. Bohne (1983b), Umweltpolitik. Band 1: Grundlagen, Analysen und Perspektiven. Opladen.

Hassenpflug, D. (1980), Umweltökonomie und Fachökologie als Gegenstände philosophischer Aufhebung zu einer Ökonomie der Natur. Eine philosophische Studie zum Ökologieproblem der Industriegesellschaft. Dissertation Gesamthochschule Kassel.

Hestenes, M. R. (1966), Calculus of Variations and Optimal Control Theory. New York.

Ho, Y.C. (1970), Differential Games, Dynamic Optimization, and Generalized Control Theory. Journal of Optimization Theory and Applications 6, 179-209.

Inagaki, M. (1970), Optimal Economic Growth. Shifting Finite Versus Infinite Time Horizon. Amsterdam, London.

Intriligator, M. D. (1964), Regional Allocation of Investment: Comment. Quarterly Journal of Economics 78, 659-662.

Intriligator, M.D. (1971), Mathematical Optimization and Economic Theory. Englewood Cliffs, N. J..

Intriligator, M. D. (1980), The Applications of Control Theory to Economics. In: A. Bensoussan und J. L. Lions (Hrsg.), Analysis and Optimization of Systems, 607-626.

Isaacs, $R$. (1965), Differential Games. New York u. a..

Jevons, W. W. (1865), The Coal Question. London.

Johnson, M. H. und J. T. Bennett (1981), Regional Environmental and Economic Impact Evaluation. An Input-Output Approach. Regional Science and Urban Economics 11, 215-230.

Kamien, M. I. und N. L. Schwartz (1981), Dynamic Optimization: The Calculus of Variations and Optimal Control in Economics and Management. New York. 
Keeler, E., M. Spence und R. Zeckhauser (1972), The Optimal Control of Pollution. Journal of Economic Theory 4, 19-34.

Klevorick, A. K. und G. H. Kramer (1973), Social Choice on Pollution Management: The Genossenschaften. Journal of Public Economics 2, 101-146.

Kneese, A. V. (1962), Water Pollution: Economic Aspects and Research Needs. Washington, D. C..

Kneese, A. V. (1964), The Economics of Regional Water Quality Management. Baltimore.

Kneese, A. V., R. U. Ayres und R. C. d'Arge (1970), Economics of the Environment. A Materials Balance Approach. Resources for the Future, Inc., Washington, D. C..

Kneese, A. V. und M. Williams (1980), Environmental Aspects of Resources Policy in a Regional Setting: The Case of Air Quality Policy in the Southwestern United States. In: H. Siebert, I. Walter und K. Zimmermann (Hrsg.), 187-206.

Krutilla, J. V. (1967), Conservation Reconsidered. American Economic Review 57, 777-786.

Krutilla, J. V. und A.C. Fisher (1975), The Economics of Natural Environments: Studies in the Valuation of Commodity and Amenity Resources. Baltimore, London.

Kyan, C. P. und J. H. Seinfeld (1973), Real-Time Control of Air Pollution. J. A. I. Ch. E. 19, 579-589.

Kydland, F . (1975), Noncooperative and Dominant Player Solutions in Discrete Dynamic Games. International Economic Review 16, 321-335.

Lange, $R$. (1973), ADPIC - A Three-Dimensional Computer Code for the Study of Pollutant Dispersal and Deposition under Complex Conditions. Report UCRL-51462. California. 
Lave, L. B. (1982), Mitigating Strategies for Carbon Dioxide Problems. Papers and Proceedings of the Ninty-Fourth Annual Meeting of the American Economic Association 72, 257-261.

Lebedeff, S. A. und S. Hameed (1975), Study of Atmospheric Transport Over Area Sources by Integral Method. Atmospheric Environment 9, 333-338.

Leitmann, G. (1974), Cooperative and Non-Cooperative Many Players Differential Games. Wien.

Leontief, W. (1970), Environmental Repercussions and the Economic Structure: An Input-Output Approach. Review of Economics and Statistics 52, 262-271.

Loehman, E. und A. Whinston (1970), The Welfare Economics of Water Resource Allocation over Time. Applied Economics 2, 75-99.

Long, N. V. und N. Vousden (1977), Optimal Control Theorems. In: J.D. Pitchford und S. J. Turnousky (Hrsg.), Applications of Control Theory to Economic Analysis. Amsterdam, 11-34.

Luenberger, D. G. (1979), Introduction to Dynamic Systems. New York. Mäler, K. G. (1974), Environmental Economics. A Theoretical Inquiry. Baltimore, London.

Malthus, T. R. (1798), First Essay on Population. New York [1966].

Marglin, S. A. (1963), The Social Rate of Discount and the Optimal Rate of Investment. Quarterly Journal of Economics 77, 95-111.

Marshall, A. (1898), Principles of Economics. Fourth Edition, London.

Martin, D. O. (1971), An Urban Diffusion Model for Estimating Long Term Average Values of Air Quality. Journal of the Air Pollution Control Association 21, 16-19. 
Mathur, V.K. (1974), Time, Affluence and Pollution in the Theory of Consumer's Behavior. Journal of Environmental Economics and Management 1, 85-95.

Meadows, D. H., D. L. Meadows, J. Randers und W. W. Behrens (1972), The Limits to Growth. New York.

Melli, P. (1976), An Application of the Galerkin Method to the EulerianLagrangian Treatment of Time-Dependent Advection and Diffusion of Air Pollutants. Princeton, N. J..

Mill, J.S. (1865), Principles of Political Economy. Reprint of the Sixth Edition, New York 1965.

Mingst, K. A. (1982), Evaluating Public and Private Approaches to International Solutions to Acid Rain Pollution. Natural Resources Journal 22, 5-20.

Mishan, E. J. (1971), The Postwar Literature on Externalities: An Interpretative Essay. Journal of Economic Literature 9, 1-28.

Montgomery, W. D. (1972), Markets in Licenses and Efficient Pollution Control Programs. Journal of Economic Theory 5, 395-418.

Munro, G. R. (1979), The Optimal Management of Transboundary Renewable Resources. The Canadian Journal of Economics 12, 355-376.

Muschett, F. D. (1982), Analyzing Atmospheric Systems. In: D. J. Basta und B. T. Bower (Hrsg.), 389-546.

Nash, J. (1951), Noncooperative Games. Annals of Mathematics 54, 286295.

Neher, P. A. (1983), Resource Allocation Rules and Long Range Planning. Discussion Paper. 
Nijkamp, P. (1978), Competition among Regions and Environmental Quality. In: W. Buhr und P. Friedrich (Hrsg.), Konkurrenz zwischen kleinen Regionen. Baden-Baden, 153-171.

Nordhaus, W. (1982), How Fast Should We Graze the Global Commons? Papers and Proceedings of the Ninty-Fourth Annual Meeting of the American Economic Association 72, 242-246.

Oden, S. (1968), The Adification of Air Precipation and its Consequences in the Natural Environment. Ecology Committee Bulletin 1, Swedish National Science Research Council, Stockholm.

Okubo, A. (1980), Diffusion and Ecological Problems: Mathematical Models. Berlin u. a..

Olson, M. (1982), Environmental Indivisibilities and Information Costs: Fanaticism, Agnosticism, and Intellectual Progress. Papers and Proceedings of the Ninty-Fourth Annual Meeting of the American Economic Association 72, 262-266.

Olson, M. und R. Zeckhauser (1967) Collective Goods, Comparative Advantage, and Alliance Efficiency. In: R. N. Mc Kean (Hrsg.), Issues in Defense Economics. New York, London, 25-48.

Olson, M. und R. Zeckhauser (1970), The Efficient Production of External Economics. American Economic Review 60, 512-517.

Organisation for Economic Co-Operation and Development OECD (Hrsg.) (1974), Problems in Transfrontier Pollution. Paris.

Organisation for Economic Co-Operation and Development OECD (Hrsg.) (1977), Legal Aspects of Transfrontier Pollution. Paris.

Osterkamp, R. und W. Schneider (1982), Zur Umweltökonomik: Einführung und Überblick. In: H. Möller, R. Osterkamp und W. Schneider (Hrsg.), Umweltökonomik. Beiträge zur Theorie und Politik, Königstein/Ts., 5-27. 
Pasquill, F. (1962), Atmospheric Diffusion. New York.

Pauly, M. V. (1970), Optimality, Public Goods, and Local Governments: A Theoretical Analysis. Journal of Political Economy 78, 572-585.

Peltzman, S. und T.N. Tideman (1972), Local versus National Pollution Control: Note. American Economic Review 62, 959-963.

Pestieau, P. (1983), Fiscal Mobility and Local Public Goods: A Survey of the Empirical and Theoretical Studies of the Tiebout Model. In: J.-F. Thisse und H. G. Zoller (Hrsg.), Locational Analysis of Public Facilities. Amsterdam.

Peterson, F. M. und A. C. Fisher (1977), The Exploitation of Extractive Resources: A Survey. The Economic Journal 87, 681-721.

Pethig, $R$. (1975), Umweltverschmutzung, Wohlfahrt und Umweltpolitik in einem Zwei-Sektoren-Gleichgewichtsmodell. Zeitschrift für Nationalökonomie 35, 99-124.

Pethig, $R$. (1976), Pollution, Welfare, and Environmental Policy in the Theory of Comparative Advantage. Journal of Environmental Economics and Management 3, 160-169.

Pethig, R. (1979), Umweltökonomische Allokation mit Emissionssteuern. Tübingen.

Pethig, $R$. (1982), Reciprocal Transfrontier Pollution. In: Global Environmental Resources, hrsg. von H. Siebert, Frankfurt, Bern, 57-93.

Pethig, $R$. (1984a), Zur intertemporalen Allokationseffizienz nicht erneuerbarer Allmendegüter. In: H. Siebert (Hrsg.), Intertemporale Allokation. Frankfurt/Main u. a. (in Vorbereitung).

Pethig, R. (1984b), International Common Property Resources. In: $D$. W. Pearce, H. Siebert und I. Walter (Hrsg.), Risk and the Political Economy of Resource Development. London.

Pigou, A. C. (1932), The Economics of Welfare. London. 
Pitchford, J.D. (1977), Two State Variable Problems. In: J.D. Pitchford und S. J. Turnousky (Hrsg.), Applications of Control Theory to Economic Analysis. Amsterdam, 127-154.

Plourde, C. G. (1972), A Model of Waste Accumulation and Disposal. Canadian Journal of Economics 5, 119-125.

Pontryagin, L. S., V. G. Boltyanskii, R. V. Gamkrelidze und E.F.Mishchenko (1962), The Mathematical Theory of Optimal Processes. New York.

Pooler, F. (1961), A Prediction Model of Mean Urban Pollution for Use with Standard Wind Roses. International Journal of Air and Water Pollution 4, 199-211.

Potier, M. (1980), Environmental Aspects of Industrial Policy in a Regional Setting. In: H. Siebert, I. Walter und K. Zimmermann (Hrsg.), 221240.

Prittwitz, V. (1983a), Europäische Zusammenarbeit in der Luftreinhaltung. Zeitschrift für Umweltpolitik 6, 117-132.

Prittwitz, V. (1983b), Umwelt und Außenpolitik. Aus Politik und Zeitgeschehen, Beilage zur Wochenzeitung Das Parlament B 42, 13-24.

Rahman, M. A. (1963), Regional Allocation of Investment. Quarterly Journal of Economics 77, 26-39.

Rahman, M. A. (1966), Regional Allocation of Investment: The Continuous Version. Quarterly Journal of Economics 80, 159-160.

Ricardo, D. (1817), The Principle of Political Economy and Taxation. London.

Runca, E., P. Melli und A. Spirito (1979), The Advection-Diffusion Model. In: International Institute for Applied Systems Analysis (Hrsg.), RealTime Forecasting of Air Pollution Episodes in the Venetian Region. Laxenburg, Österreich, 1-20. 
Runca, E., P. Melli und P. Zanetti (1976), Computation of Long-Term Average $\mathrm{SO}_{2}$ Concentrations in the Venetian Area. Applied Mathematical Modelling. Environmental, Social and Engineering Systems 1, 9-15.

Sandler, T. und A. J. Culyer (1982a), Joint Products and Inter-Jurisdictional Spillovers: Some Public Geometry. Kyklos 35, 702-709.

Sandler, T. und A.J. Culyer (1982b), Joint Products and Multijurisdictional Spillovers. Quarterly Journal of Economics 96, 707-716.

Sandler, T. und J. T. Tschirhart (1980), The Economic Theory of Clubs. An Evaluative Survey. Journal of Economic Literature 18, 1481-1521.

Schotter, A. und G. Schwödiauer (1980), Economics and Game Theory: A Survey. Journal of Economic Literature 18, 479-527.

Schulze, W.D. (1974), The Optimal Use of Non-Renewable Resources: The Theory of Extraction. Journal of Environmental Economics and Management 1, 53-73.

Seinfeld, J. H. (1975), Air Pollution - Physical and Chemical Fundamentals. New York.

Sharma, V. und L. O. Myrup (1975), Diffusion from a Line Source in an Urban Atmosphere. Atmos. Environ. 9, 907-922.

Siebert, $H$. (1975), Regional Aspects of Environmental Allocation.

Zeitschrift für die gesamte Staatswissenschft 131, 496-513.

Siebert, $H$. (1977), Environmental Quality and the Gains from Trade. Kyklos 30, 657-673.

Siebert, H. (1978), Ökonomische Theorie der Umwelt. Tübingen.

Siebert, H. (1979a), Environmental Policy in the Two-Country-Case. Zeitschrift für Nationalökonomie 39, 259-274.

Siebert, H. (Hrsg.) (1979b), Umwelt und wirtschaftliche Entwicklung. Darmstadt. 
Siebert, H. (1980a), The Dynamic Aspects of Environmental Allocations: Comment. In: H. Siebert, I. Walter und K. Zimmermann (Hrsg.), 148151.

Siebert, H. (1980b), The Regional Dimensions of Environmental Policy. In:

H. Siebert, I. Walter und K. Zimmermann (Hrsg.), 1-12.

Siebert, H. (1981), Economics of the Environment. Lexington, Mass. und Toronto.

Siebert, $H$. (1983a), Marktwirtschaftliche Instrumente im Umweltschutz. Unveröffentlichtes Vortragsmanuskript.

Siebert, H. (1983b), Ökonomische Theorie natürlicher Ressourcen. Tübingen.

Siebert, H. (1984), Spatial Aspects of Environmental Economics.In: A. V. Kneese und J. L. Sweeney (Hrsg.), Handbook of Natural Resource and Energy Economics. Band 1, Amsterdam (in Vorbereitung).

Siebert, H., J. Eich.berger, R. Gronych und R. Pethig (1980), Trade and Environment. A Theoretical Enquiry. Amsterdam.

Siebert, H., I. Walter und K. Zimmermann (Hrsg.) (1980), Regional Environmental Policy. The Economic Issues. London, Basingstoke.

Sklarew, R. C., A. J. Fabrick und J. A.Prager (1971), A Particle-in-Cell Method for the Numerical Solution of the Atmospheric Diffusion Equation, and Application to Air Pollution Problems. Report 3SR-844, Systems, Science and Software, La Jolla, California.

Smets, H. (1974), Alternative Economic Policies of Unidirectional Transfrontier Pollution. In: OECD (Hrsg.), Problems in Transfrontier Pollution. 75-146.

Smith, V.L. (1972), Dynamics of Waste Accumulation: Disposal versus Recycling. Quarterly Journal of Economics 86, 600-616. 
Snower, D. (1982), Policy-Target Dynamics in a Global-Model Context. In: G. Bruckmann (Hrsg.), Environmental Aspects in Global Modelling. Laxenburg, Österreich, 295-308.

Der Rat von Sachverständigen für Umweltfragen SRU (1974), Umweltgutachten. Wiesbaden.

Der Rat von Sachverständigen für Umweltfragen SRU (1978), Umweltgutachten. Stuttgart, Mainz.

Der Rat von Sachverständigen für Umweltfragen SRU (1983), Sondergutachten Waldschäden und Luftverunreinigungen. Stuttgart, Mainz.

Stahl, K. und P. Varaiya (1983), Local Collective Goods: A Critical ReExamination of the Tiebout Model. In: J.-F. Thisse und H. G. Zoller (Hrsg.), Locational Analysis of Public Facilities. Amsterdam.

Starr, A. W. und Y.C. Ho (1969a), Nonzero-Sum Differential Games. Journal of Optimization Theory and Application 3, 184-206.

Starr, A. W. und Y. C. Ho (1969b), Further Properties of Nonzero-Sum Differential Games. Journal of Optimization Theory and Application 3, 207-219.

Starrett, D. A. (1978), Market Allocations of Location Choice in a Model with Free Mobility. Journal of Economic Theory 17, 21-37.

Stein, J. L. (1971), The 1971 Report of President's Council of Economic Advisers: Microeconomic Aspects of Public Policy. American Economic Review 61, 531-537.

Stern, A. C. (Hrsg.) (1976), Air Pollution. New York u. a., 5 Bände.

Stiglitz, J. E. (1977), The Theory of Local Public Goods. In: M. S. Feldstein und R. P. Irman (Hrsg.), TheEconomics of Public Service. London, 274333.

Sutton, O. G. (1953), Micrometeorology. New York. 
Takayama, A. (1974), Mathematical Economics. Hinsdale, Ill..

Tennekes, H. (1976), Observations on the Dynamics and Statistics of Simple Box Models with a Variable Inversion Ltd. In: Third Symposium on Atmospheric Turbulence, Diffusion and Air Quality, Boston, 397-402.

Tiebout, C.M. (1956), A Pure Theory of Local Public Goods. Journal of Political Economy 64, 416-424.

Tietenberg, $T$. (1974), On Taxation and the Control of Externalities: Comment. American Economic Review 64, 462-466.

Toussaint, S. (1984), Notwendige Optimalitätsbedingungen in der Kontrolltheorie. In: H. Siebert (Hrsg.), Intertemporale Allokation. Frankfurt/Main u. a. (in Vorbereitung).

Victor, P. (1972), Pollution: Economy and Environment. Toronto.

Vogt, W. (1981), Zur intertemporal wohlfahrtsoptimalen Nutzung knapper, natürlicher Ressourcen. Eine kontrolltheoretische Analyse. Tübingen.

Waite, T.D. und N. J. Freeman (1977), Mathematics of Environmental Processes. Lexington/Massachussetts, Toronto.

Walter, I. (1975), International Economics of Pollution Control. New York, Toronto.

Weinstein, M. C. und R. J. Zeckhauser (1974), Use Patterns for Depletable and Recycleable Resources. Review of Economic Studies, Symposium on the Economics of Exhaustible Resources, 67-88.

Westhoff, F . (1977), Existence of Equilibria in Economics with a Local Public Good. Journal of Economic Theory 14, 84-112. 
Wetstone, G. und A. Rosencranz (1982), Acid Rain in Europe and North America: National Responses to an International Problem. Final Report (Draft). A Study for the German Marshall Fund of the United States.

Wooders, $M$. (1978), Equilibria, the Core, and Jurisdiction Structures in Economies with a Local Public Good. Journal of Economic Theory 18, 328-348.

Wooders, M. (1980), The Tiebout Hypotheses: New Optimality in Local Public Good Economies. Econometrica 48, 1467-1485.

Wright, C. (1974), Some Political Aspects of Pollution Control. Journal of Environmental Economics and Management 1, 173-186.

van Zele, $R$. (1978), An International (Interjurisdictional) Analytical Framework for Environmental Management. New York. 
Helga Gebauer - 978-3-631-75599-0

Downloaded from PubFactory at 01/11/2019 03:14:13AM

via free access 


\section{STAATLICHE ALLOKATIONSPOLITIK IM MARKTWIRTSCHAFTLICHEN SYSTEM}

Band 1 Horst Siebert (Hrsg.): Umweltallokation im Raum. 1982.

Band 2 Horst Siebert (Hrsg.): Global Environmental Resources. The Ozone Problem. 1982.

Band 3 Hans-Joachim Schulz: Steuerwirkungen in einem dynamischen Unternehmensmodell. Ein Beitrag zur Dynamisierung der Steuerüberwälzungsanalyse. 1981.

Band 4 Eberhard Wille (Hrsg.): Beiträge zur gesamtwirtschaftlichen Allokation. Allokationsprobleme im intermediären Bereich zwischen öffentlichem und privatem Wirtschaftssektor. 1983.

Band 5 Heinz König (Hrsg.): Ausbildung und Arbeitsmarkt. 1983.

Band 6 Horst Siebert (Hrsg.): Reaktionen auf Energiepreissteigerungen. 1982.

Band 7 Eberhard Wille (Hrsg.): Konzeptionelle Probleme öffentlicher Planung. 1983.

Band 8 Ingeborg Kiesewetter-Wrana: Exporterlösinstabilität. Kritische Analyse eines entwicklungspolitischen Problems. 1982.

Band 9 Ferdinand Dudenhöffer: Mehrheitswahl-Entscheidungen über Umweltnutzungen. Eine Untersuchung von Gleichgewichtszuständen in einem mikroökonomischen Marktund Abstimmungsmodell. 1983.

Band 10 Horst Siebert (Hrsg.): Intertemporale Allokation. 1984.

Band 11 Helmut Meder: Die intertemporale Allokation erschöpfbarer Naturressourcen bei fehlenden Zukunftsmärkten und institutionalisierten Marktsubstituten. 1984.

Band 12 Ulrich Ring: Offentliche Planungsziele und staatliche Budgets. Zur Erfüllung öffentlicher Aufgaben durch nicht-staatliche Entscheidungseinheiten. 1985.

Band 13 Ehrentraud Graw: Informationseffizienz von Terminkontraktmärkten für Währungen. Eine empirische Untersuchung. 1984.

Band 14 In Vorbereitung.

Band 15 In Vorbereitung.

Band 16 Helga Gebauer: Regionale Umweltnutzungen in der Zeit. Eine intertemporale ZweiRegionen-Analyse. 1985. 
Siebert, Horst (Hrsg.)

\section{UMWELTALLOKATION IM RAUM}

Frankfurt/M., Bern, 1982. V, 232 S.

Staatliche Allokationspolitik im marktwirtschaftlichen System. Bd. 1

ISBN 3-8204-5997-9

br. sFr. 45.-

In diesem Sammelband werden Beiträge zusammengefasst, die sich mit der Nutzung der Umwelt in ihren verschiedenen Funktionen (öffentliches Konsumgut, Rezeptor von Schadstoffen) unter dem besonderen Aspekt der räumlichen Dimensionen von Umweltgütern befassen. Der Band enthält wirtschaftspolitisch und theoretisch ausgerichtete Beiträge. Insbesondere wird versucht, die Bestimmung der anzustrebenden Umweltqualität in räumlichen Systemen (Regionen) und die für die Erreichung dieser Umweltqualität einzusetzenden Instrumente zu erklären.

Aus dem Inhalt: Wirkungsweisen umweltpolitischer Instrumente (Siebert) - Umwelt- und Regionalpolitik in der Bundesrepublik Deutschland (Vogt) - Bestimmung des anzustrebenden Umweltqualitätswertes (Dehez) - Allokation öffentlicher Güter bei Konsumentenmobilität (Dudenhöffer/Gebauer) - Mehrheitswahl als Instrument regionalisierter Umweltpolitik (Dudenhöffer) Intertemporale regionale Umweltallokation (Gebauer).

Siebert, Horst (Hrsg.)

\section{REAKTIONEN AUF ENERGIEPREISSTEIGERUNGEN}

Frankfurt/M., Bern, 1982. $138 \mathrm{~S}$.

Staatliche Allokationspolitik im marktwirtschaftlichen System. Bd. 6

ISBN 3-8204-7254-1

br. sFr. 31.-

Dieser Sammelband fasst vier Beiträge zur Anpassung der Industrienationen auf Energieverknappungen und Energiepreiserhöhungen zusammen. Die Beiträge gehen von den Preissteigerungen für Erdöl 1973/74 und 1979/80 aus und untersuchen, welche Inzidenz diese Preissteigerungen auf makroökonomische Variable wie Preisniveaustabilität, Zahlungsbilanzsituation, Beschäftigung und Sektorstruktur haben und welche Anpassungen in den Industrienationen erfolgt sind oder vorgenommen werden sollten.

Aus dem Inhalt: Die Anpassung der Volkswirtschaft beim Ubergang zu neuen Energieversorgungssystemen: Die theoretische Grundlage eines Energiemodells für die Bundesrepublik Deutschland (Conrad) - Importieren wir Stagflation über steigende Rohstoffpreise? Keynesianische und monetaristische Ansichten (Schmid) - Absatzsteuem, Olförderung und das Allmendeproblem (Sinn) - Energiepreisentwicklung und Konsumallokation privater Haushalte (Zimmermann).

\section{Verlag Peter Lang Bern $\cdot$ Frankfurt a.M. New York}

Auslieferung: Verlag Peter Lang AG, Jupiterstr. $15, \mathrm{CH}-3000$ Bern 15 Telefon $(0041 / 31) 321122$. Telex verlch 32420

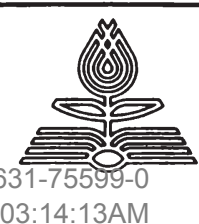

